\title{
Function of the $\alpha / \beta$-hydrolase fold family proteins Pummelig (CG1882) and Hormone-sensitive lipase in the Drosophila melanogaster lipid metabolism
}

\author{
Dissertation \\ for the award of the degree \\ Doctor rerum naturalium \\ of the Georg-August-Universität Göttingen \\ within the doctoral program Genes and Development \\ of the Georg-August University School of Science (GAUSS) \\ submitted by \\ Philip Hehlert \\ from Berlin
}

Göttingen, 2016 


\section{Thesis Committee}

Prof. Dr. Ernst Wimmer,

Dept. of Developmental Biology, Georg August University Göttingen

PD Dr. Ronald P. Kühnlein,

Research group Molecular Physiology, Max Planck Institute for biophysical

Chemistry

Assoz. Prof. Dr. Robert Zimmermann

Research group Molecular Enzymology, Institute of Molecular Biosciences (IMB) at the University Graz

\section{Members of the Examination Board}

Referee: $\quad$ Prof. Dr. Ernst Wimmer,

Dept. of Developmental Biology, Georg August Universität Göttingen

$2^{\text {nd }}$ Referee: PD Dr. Ronald P. Kühnlein,

Research group Molecular Physiology, Max Planck Institute for biophysical Chemistry

(if applicable) $3^{\text {rd }}$ referee: Prof. Ahmed Mansouri,

Molecular Cell Differentiation, Max Planck Institute for biophysical Chemistry

\section{Further members of the Examination Board}

Dr. Roland Dosch

Dept. of Developmental Biochemistry, Universitätsmedizin (UMG) Göttingen

Assoz. Prof. Robert Zimmermann

Research group Molecular Enzymology, Institute of Molecular Biosciences (IMB) at the University Graz

Prof. Dr. Ivo Feußner

Dept. Biochemistry of the plant, Georg-August-University Göttingen

Date of oral examination: $3^{\text {rd }}$ of August 2016 


\section{Danksagung}

Diese Arbeit wurde am Max-Planck-Institut für biophysikalische Chemie (Göttingen) in der Arbeitsgruppe Molekulare Physiologie (in der Abteilung Molekulare Entwicklungsbiologie) unter der Betreuung von PD Dr. Ronald P. Kühnlein durchgeführt. Ich danke Prof. Jäckle für die Gelegenheit meine Dissertation in seiner Abteilung, mit ihren ausgezeichneten Bedingungen, durchführen zu können.

Mein besonderer Dank gilt PD Dr. Ronald Kühnlein für seine fortwährende Unterstützung und seine große und kritische Diskussions- und Hilfsbereitschaft, die zum Gelingen dieser Arbeit beigetragen haben. Außerdem bedanke ich mich für die Möglichkeit meine Dissertation in weiten Teilen selbständig gestalten und parallel noch Beiträge für Publikationen außerhalb meiner Promotion beisteuern zu können.

Weiter Dank gilt auch meinem erweiterten Betreuungsausschuss um Prof. Wimmer und Prof. Zimmermann für ihr ständiges Interesse an meiner Arbeit und den richtungsgebenden Diskussionen.

Im Besonderen danke ich dabei Prof. Zimmermann, für die Möglichkeit Experimente in seiner Arbeitsgruppe in Graz durchführen zu können. Großer Dank gilt auch Prof. Monika Oberer für die Möglichkeit in ihrer Arbeitsgruppe in Graz Experimente machen zu können.

Meinen Kooperationspartnern Dr. Christoph Heier, Dr. Harald Nagy und Lisa Maresch danke ich für Ihre Unterstützung bei den Experimenten in Graz.

Des Weiteren möchte ich meinen Kooperationspartnern Dr. Thomas Eichmann und Vinzenz Hofferek für die Zusammenarbeit bei den Lipidomics-Analysen danken. Dr. Dietmar Riedel gilt mein Dank für die elektronenmikroskopischen Aufnahmen. Großen Dank auch an Dr. Vlad Pena und Dr. Inessa De für die Möglichkeit der Nutzung ihrer Zellkulturräume am MPI-BPC.

Vielen Dank auch an alle aktuellen und ehemaligen Mitgliedern der Arbeitsgruppe Molekulare Physiologie. Im besonderen Iris Bickmeyer und Olga Babosova für ihre Unterstützung bei einigen Experimenten und ihren Beiträgen zu einer guten Laboratmosphäre.

Dr. Sebastian Grönke, Anna Takacs, Dr. Anja Hildebrandt und Jonathan Rosenberg danke ich für ihre Vorarbeiten am Hsl- und Pummelig-Projekt. Dr. Anita Sahu-Ohsen und Assoz. Prof. Dr. Ruth Birner-Grünberger danke ich für ihre Möglichkeit am Cyp1-Projekt mitwirken zu können.

Bei allen ehemaligen und aktuellen Mitarbeitern der Abteilung Molecular Developmental Biology möchte ich mich herzlich bedanken für die gute Atmosphäre, die diesen Ort zu wirklich etwas Speziellen machen! Im Besonderen gilt mein Dank Dr. Ralf Pflanz für seine erweiterte Diskussionsbereitschaft und Input sowie Material für Experimente, aber auch das durchsehen von Teilen meiner Dissertation. Besonderen Dank auch an Dr. Annekatrin König für ihre vielen Kommentare zu meiner Dissertation und das diskutieren vieler Aspekte.

Des Weiteren danke ich meinen Freunden Iro und seiner Familie, Ralf und Andres für die schöne Zeit hier in Göttingen, die langen Gespräche und manchmal sogar einen vollen Kühlschrank. Natürlich auch meinen Volleyballern vom TSV Roringen, die einen geistig und körperlich fordern und die die schon so kurze Zeit hier noch kürzer erscheinen lassen.

Zuletzt möchte ich meinen Eltern und meinem Bruder und natürlich meinen Großeltern danken für ihre ständige Unterstützung, ihr Verständnis und ihre Geduld. 


\section{Fruchtfliegen}

\section{Danke für Ihre Mitarbeit!}

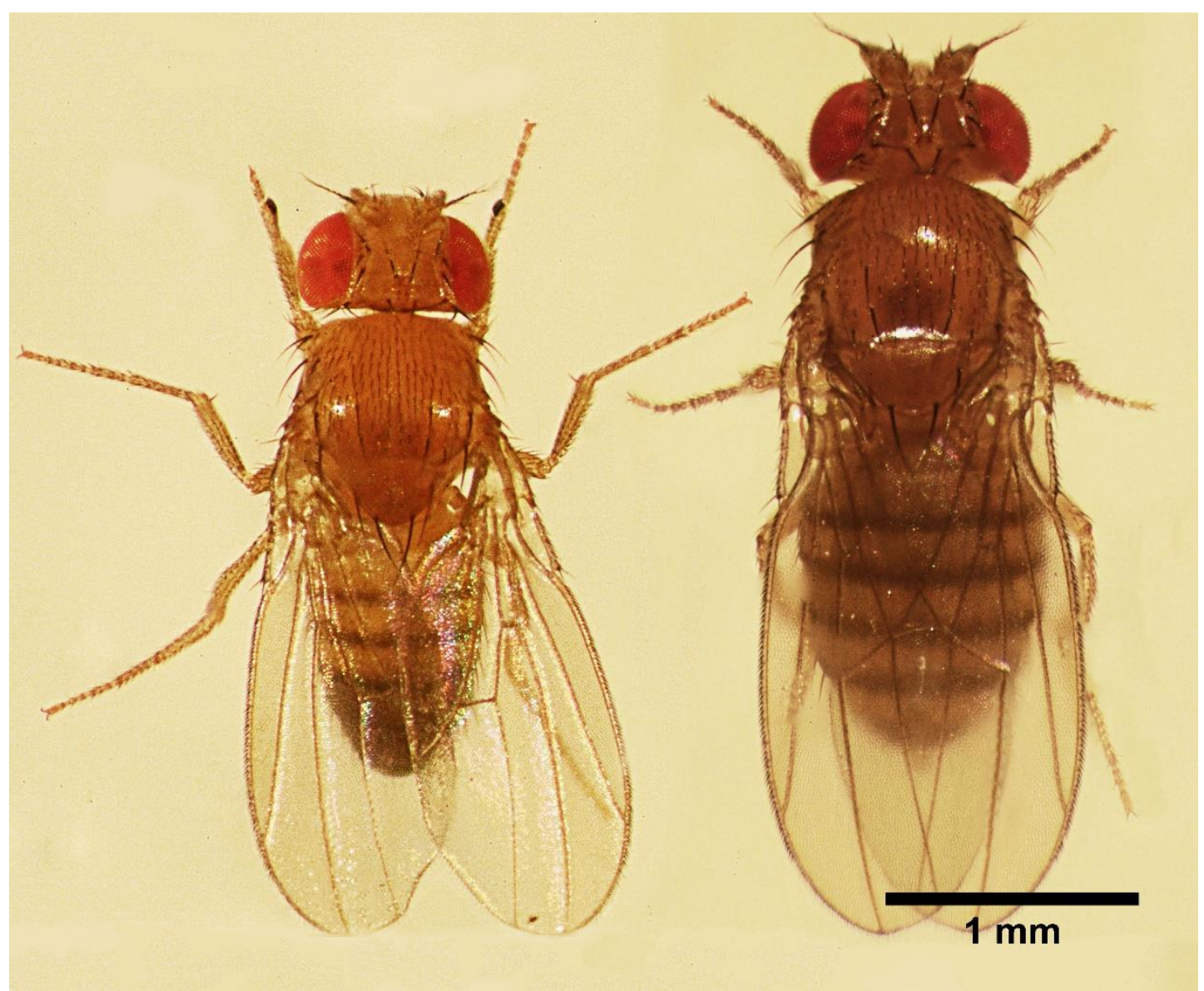

These are Kathryn and Jean-Luc two average fruit flies (female right, male left). 


\section{Summary}

To maintain energy homeostasis, all organisms need to adjust the generation and mobilization of their energy stores. The key molecules for energy storage are neutral lipids, mainly triacylglycerides (TAGs), which accumulate in specialized tissues like the mammalian adipose tissue or the fat body of the fruit fly Drosophila melanogaster. Inside the cell neutral lipids are wrapped by phospholipid monolayer to form a unique organelle called lipid droplet (LD). A set of LD proteins act on the surface of these organelles to manage fundamental lipid homeostasis functions like lipid mobilization at this compartment border.

Remarkably, central mammalian LD proteins involved in storage fat mobilization like Perilipins or the Adipose triglyceride lipase (ATGL) have functional homologues in fruit flies (namely Plin1 and Brummer) suggesting an evolutionary conservation of factors and mechanisms of lipid mobilization between flies and men. In mammals the $\alpha / \beta$-hydrolase fold family proteins Hormone-sensitive lipase (HSL) and $\alpha / \beta$-hydrolase domain containing 5 (ABHD5 or CGI-58) are core components of the lipid mobilization module. ABHD5 acts as an activator of ATGL and HSL represents the main diacylglyceride (DAG) lipase.

In this work I characterized the functions of the related genes for mammalian $H S L(H s /)$ and $A B H D 5$ (CG1882, pummelig) in D. melanogaster.

Most findings for $\mathrm{Hsl}$, are consistent with the published data for its mammalian homolog indicating an evolutionary conservation of its function. $\mathrm{DmHs}{ }^{1}$ mutant flies have no altered body fat storage, as also observed in HSL deficient mice. A DmHsl::GFP fusion protein is conditionally localized on LDs and the substrate spectrum is very similar to mammalian HSL. However, whereas diacylglyceride amounts are increased in HSL deficient mice, this could not be observed in $\mathrm{DmHs}^{1}$ mutant flies. Also neither lipid mobilization nor fecundity were impaired in $\mathrm{DmHsl}$ deficient flies, leaving it open to identify a biological phenotype in $\mathrm{DmHs} \mathbf{l}^{1}$ flies.

pummelig mutant (pum/ ${ }^{1}$ ) larvae had normal body fat storage but body fat stores (mainly TAGs) in adult pum ${ }^{1}$ flies were increased in comparison to control flies. At the same time Glycogen stores in pum $I^{1}$ flies were decreased by $\sim 40 \%$ compared to control flies which was accompanied by a higher desiccation sensitivity. pum ${ }^{1}$ flies survived significantly longer under starvation and surprisingly mobilized storage lipids faster than controls. In vitro assays using recombinantly expressed pummelig identified Puml as an active phospholipase with substrate affinities for Phosphatidic acid (PA), Phosphatidylglycerol (PG), N-Arachidonoyl-phosphatidylethanolamine (NAPE), Ethyl palmitate and Bis(monoacylglycero)phosphate (BMP $[R, R]$ ). However, Puml cannot activate the main triglyceride lipase Brummer in flies.

Besides increased body fat storage, massive lipid accumulations in Malpighian tubules (the renal organs of the fly) could be observed in pum $l^{1}$ flies. Further experiments indicated a tissue autonomous control of lipid storage in Malpighian tubules. Additionally, metabolic rate in pum ${ }^{1}$ flies was similar to control flies. Interestingly, food intake of puml ${ }^{1}$ flies was comparable to controls but the rate of lipogenesis was drastically increased.

Localization studies using Puml::mCherry fusion protein confirmed the LD localization in adult fat body tissue and additionally could show that Puml::mCherry co-localized with peroxisome-targeted eYFP. As peroxisomes are important for the breakdown of long-chain fatty acids (LCFAs) a lipidomics analysis was performed with Malpighian tubule samples that revealed increased TAG storage with a shift towards longer fatty acid sidechains and increased un-saturation grade of the esterified fatty acids. An extended working model is provided which explains the observed phenotypes in pum/ ${ }^{1}$ flies. My findings contribute to a broader understanding of the complex network which controls lipid metabolism. 


\section{Table of Content}

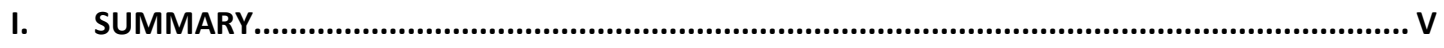

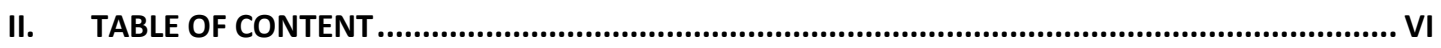

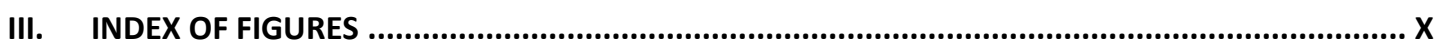

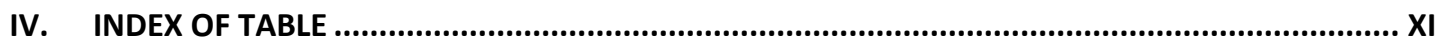

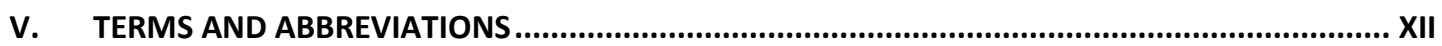

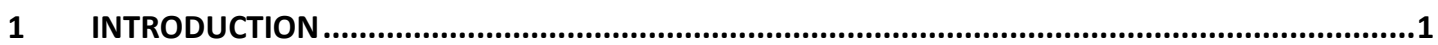

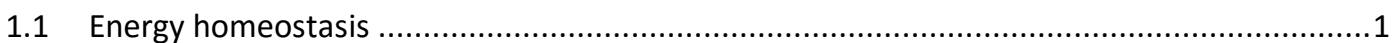

1.2 Lipid storage regulation in mammals ...................................................................

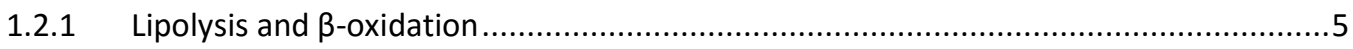

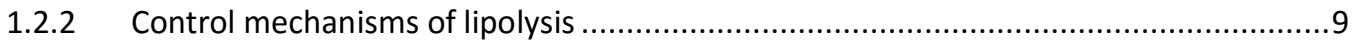

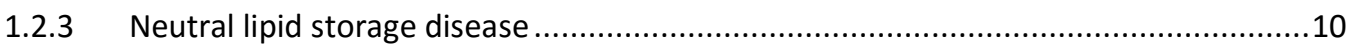

1.3 Drosophila a model system for lipid research ............................................................. 11

1.3.1 Lipid mobilization in Drosophila melanogaster..................................................15

1.3.2 Pummelig the single sequence related protein to mammalian $\alpha / \beta$-hydrolase domain containing 4 and 5 in Drosophila melanogaster................................................ 18

1.3.3 Genomic locus of pummelig and Puml constructs ................................................21

1.3.4 Main findings from previous contributors to the characterization of pummelig ........21

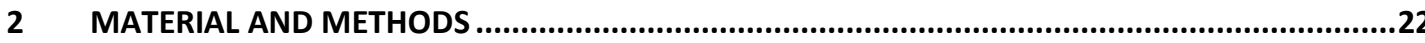

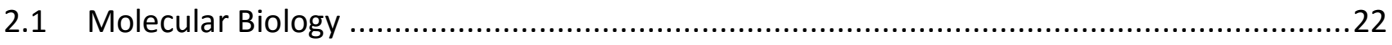

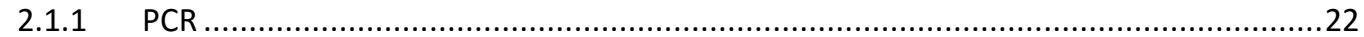

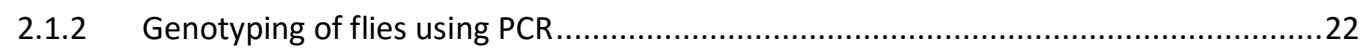

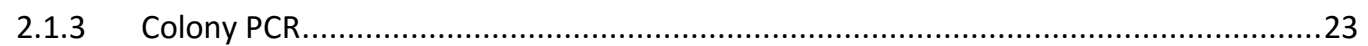

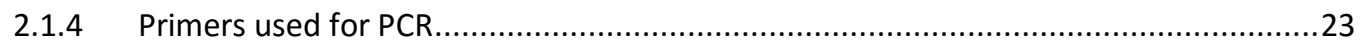

2.1.5 Reverse transcribed quantitative Polymerase Chain reaction (RT-qPCR) for gene

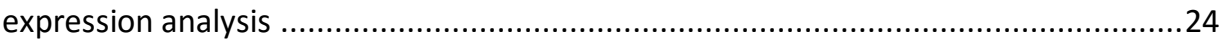

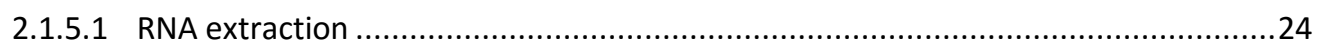

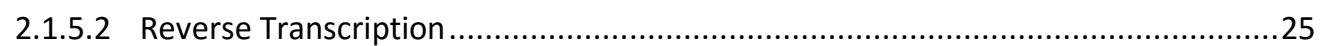

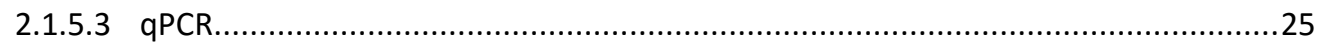

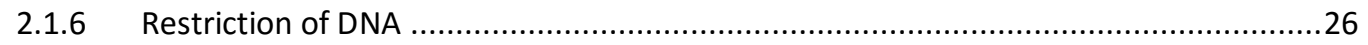

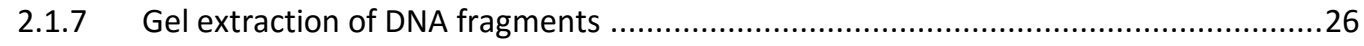

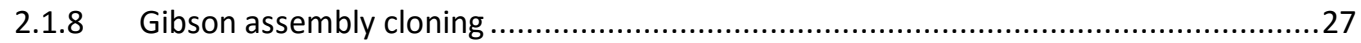

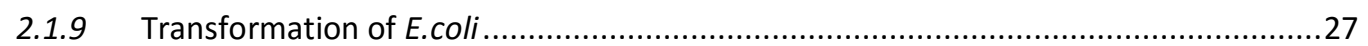

2.1.9.1 Transformation with chemically competent cells..............................................27

2.1.9.2 Transformation with electro competent cells ....................................................28

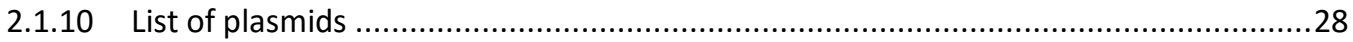

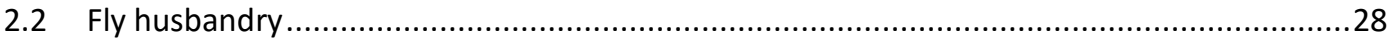

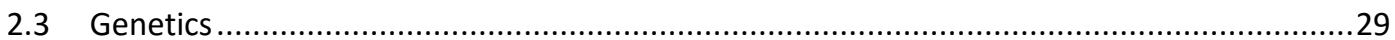


2.3.1 Ectopic gene expression via the GAL4/UAS-System .............................................29

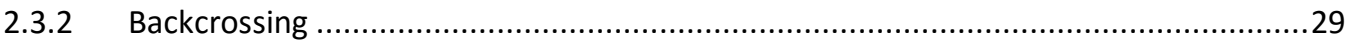

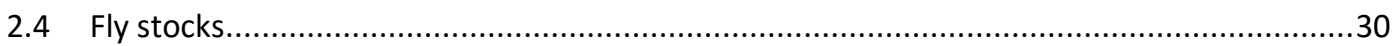

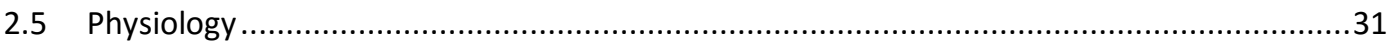

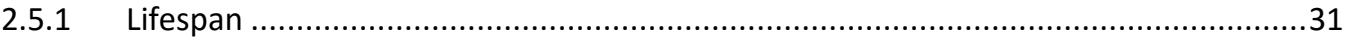

2.5.2 Capillary feeding (CAFÉ) assay for quantification of food uptake ............................32

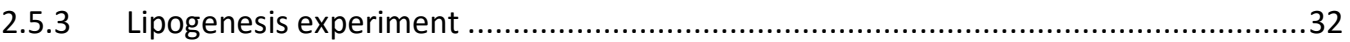

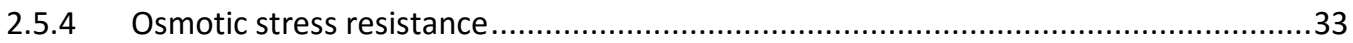

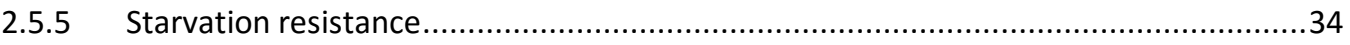

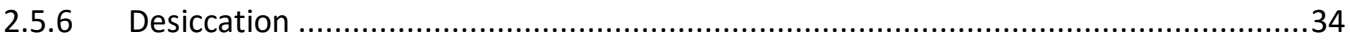

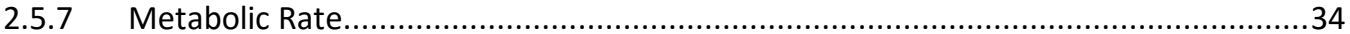

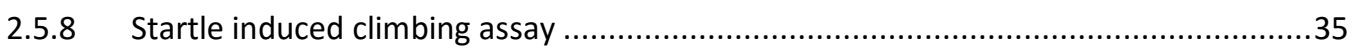

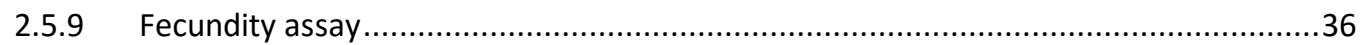

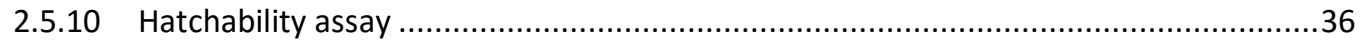

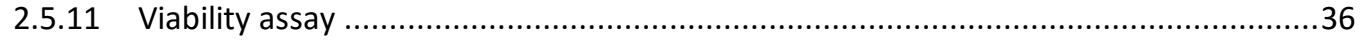

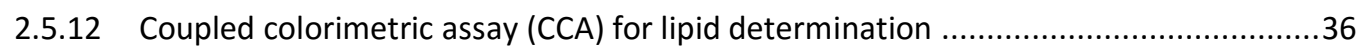

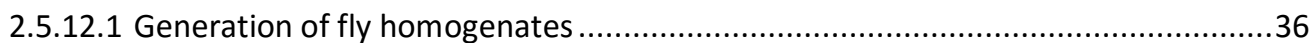

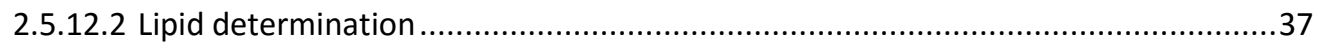

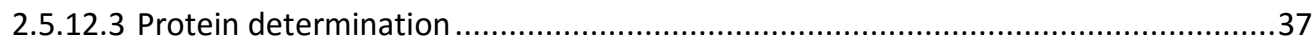

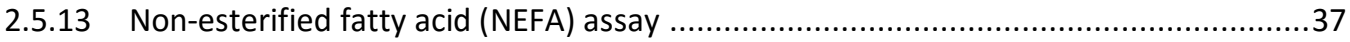

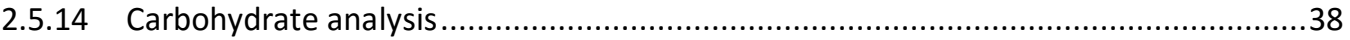

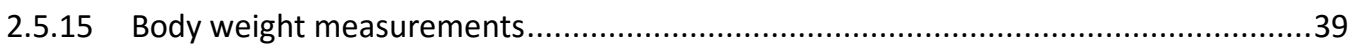

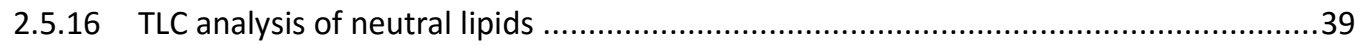

2.5.17 Lipidomics analysis of Malpighian tubules ....................................................40

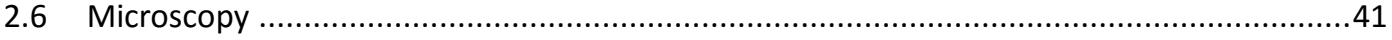

2.6.1 Used fluorophores, dyes and concentrations ..................................................41

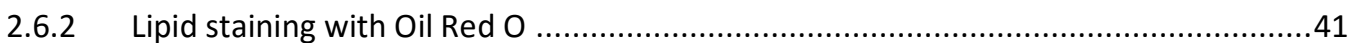

2.6.3 Imaging of Malpighian tubules and gut-ring fat body ......................................42

2.6.4 Imaging of adult cuticle attached fat body ................................................43

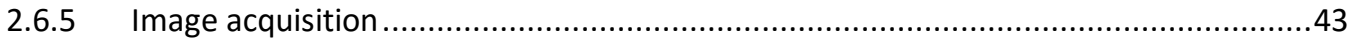

2.6.6 Lipid Droplet Size quantification.................................................................43

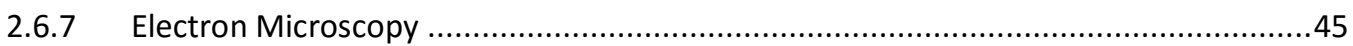

2.6.8 Measurement of Mitochondrial diameter .................................................... 45

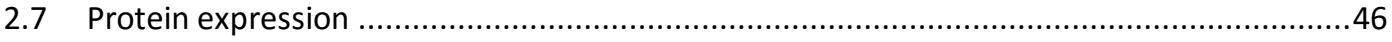

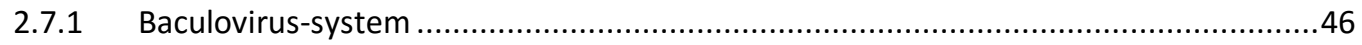

2.7.1.1 Virus production and protein expression in Sf-9 cells ......................................46

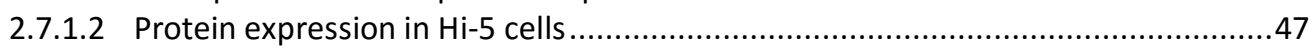

2.7.2 E.coli expression system ............................................................................... 48

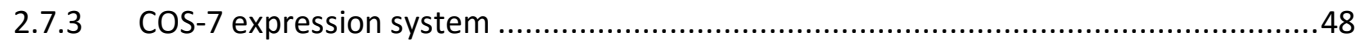


2.8 Lysate preparation for recombinantly expressed proteins

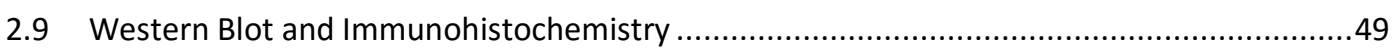

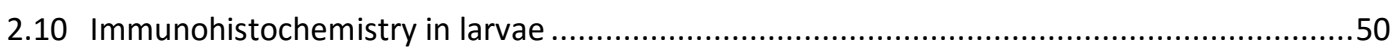

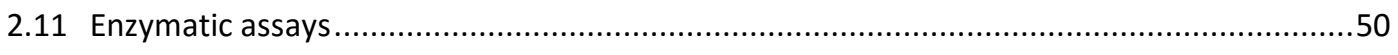

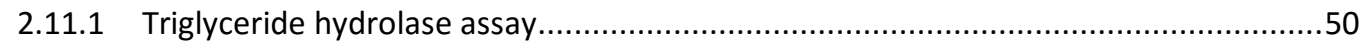

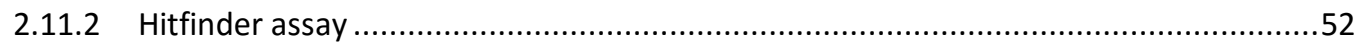

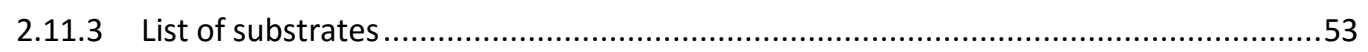

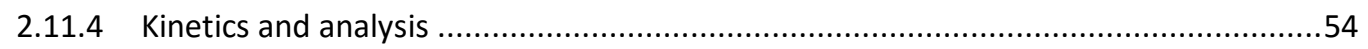

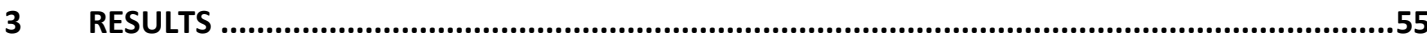

3.1 Body fat storage in pummelig mutant flies .............................................................55

3.1.1 Lipid storage is increased in pummelig mutants .............................................55

3.1.2 pummelig and brummer mutants are obese but not overweight ...........................57

3.1.3 Body fat over-storage can be observed in pummelig mutant flies but not larvae .......57

3.2 Mean life time is decreased in pummelig mutant flies ..................................................58

3.3 Pummelig a starvation-responsive gene ...................................................................59

3.3.1 Expression of pummelig is increased under starvation ........................................59

3.3.2 Starvation resistance is enhanced in pummelig mutant flies .................................60

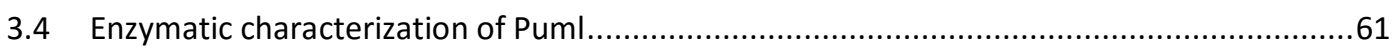

3.4.1 Puml is not the activator of $\mathrm{Bmm}$ and has no triacylglyceride hydrolase activity .......61

3.4.2 Recombinant Puml has phospholipase activity ................................................62

3.5 Energy storage of pummelig mutant flies under fed and stress conditions .......................64

3.5.1 Glycerolipid consumption under starvation is higher in pummelig mutants compared to control flies .64

3.5.2 Metabolic rate is not changed in pummelig mutant flies ......................................66

3.5.3 Glycogen storage is decreased in pummelig mutant flies ......................................66

3.5.4 Desiccation resistance is impaired in pummelig mutant flies..................................67

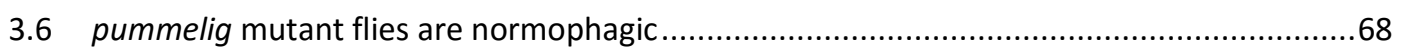

3.7 Lipogenesis is increased in pummelig mutant flies ......................................................69

3.8 Localization of Puml and lipid storage phenotypes in pummelig mutant flies ....................71

3.8.1 Puml is a member of the lipid droplet proteome.................................................71

3.8.2 pummelig mutant flies exhibit ectopic lipid storage in Malpighian tubules .................72

3.8.2.1 pummelig expression in pummelig mutant flies can rescues the lipid over-storage

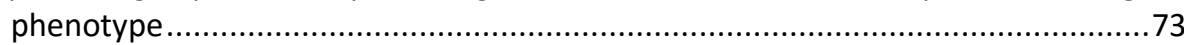

3.8.3 Pummelig::mCherry fusion protein is localized on peroxisomes .............................75

3.9 Lipid over-storage in Malpighian tubules of pummelig mutant flies does not impair osmotic

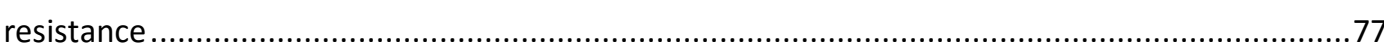

3.9.1 Lipid droplet distribution is altered in pummelig mutant flies ...............................77 
3.9.2 Long-chain fatty acids and poly-unsaturated fatty acids are elevated in pummelig mutant flies

4.1 Localization, interactions and structure of Pummelig ..................................................8 85

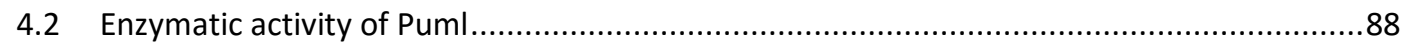

4.3 Lipogenesis in pummelig mutants ......................................................................91

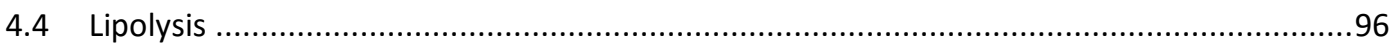

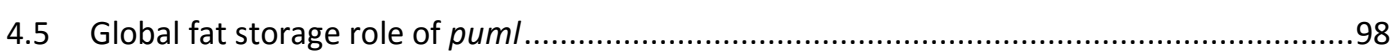

4.6 A new insight in lipid storage control in Drosophila .................................................103

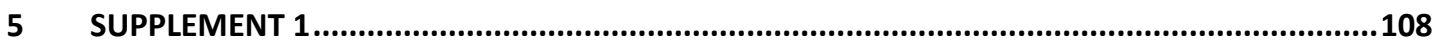

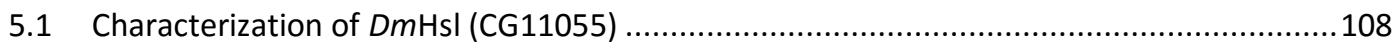

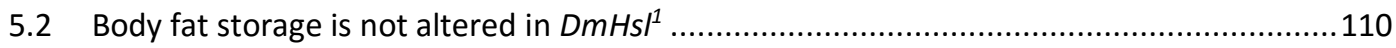

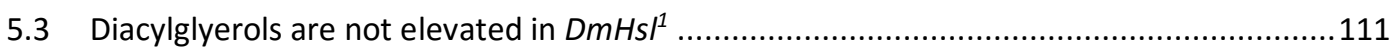

$5.4 D m H s \mid:: E G F P$ abundance on LDs is higher during starvation in larvae and adults ...............112

5.5 Lipid mobilization in $\mathrm{DmHs}^{1}$ flies is not impaired ...................................................114

5.6 DmPlin1 is crucial for localization of $D m H$ sl::GFP on large LDs $(>10 \mu m)$ but not small .......115

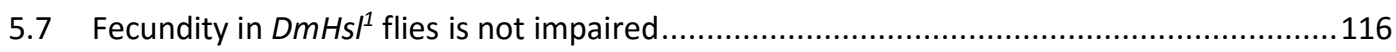

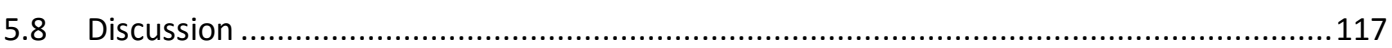

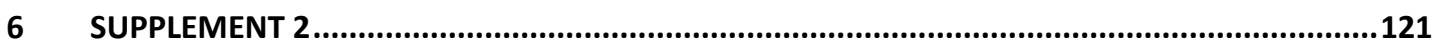

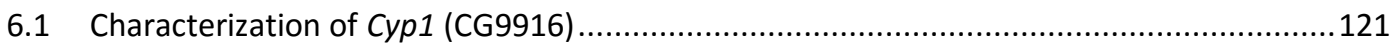

6.2 Cyp1::eGFP can be associated with LDs ................................................................ 122

6.3 Average lipid droplet size is decreased in $C y p 1^{1}$ flies ...................................................123

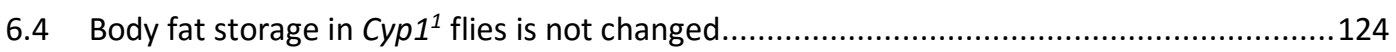

6.5 Cyp1::eGFP expression in larvae reverts small LD phenotype ..........................................125

6.6 Cyp1::eGFP overexpression in larval fat body does not enhance giant LD phenotype of plin $1^{1}$

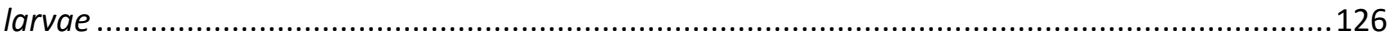

6.7 Cyp1 contributes to lipid droplet size and storage lipid partioning in plin $1^{1}$ larvae ...........127

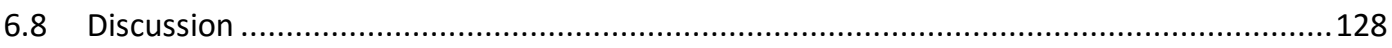

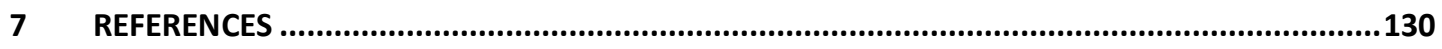

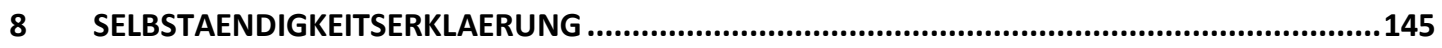

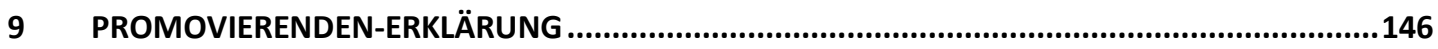

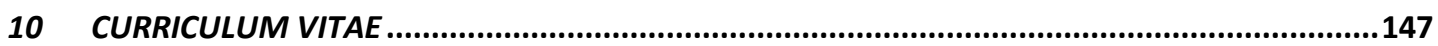




\section{Index of figures}

Figure 1 Factors that influence energy balance and control weight ..........................................1

Figure 2 Physiological systems that regulates energy stores ..................................................

Figure 3 Schematic illustration of the lipid droplet structure ................................................ 5

Figure 4 Schematic overview of mammalian lipid mobilization for ATP regeneration. ......................6

Figure 5 Schematic overview of lipid breakdown for ATP synthesis and its regulation .......................8

Figure 6 Schematic overview of intra-cellular energy balance regulation in mammals ......................10

Figure 7 Life cycle of Drosophila melanogaster and distribution of the main lipid storage tissue the fat body in flies.

Figure 8 Schematic overview of the generation of neutral and phospholipids in Drosophila melanogaster...

Figure 9 Schematic overview of storage lipid mobilization in Drosophila melanogaster for ATP synthesis.

Figure 10 Phylogenetic analysis of Puml, conservation of the catalytic center and PKA phosphorylation site

Figure 11 In silico prediction of phosphorylation sites in Puml ......................................................20

Figure 12 Genomic locus of puml, constructs and proteins available........................................21

Figure 13 Interference with $\mathrm{bmm}$ and pum/ can lead to changes in body fat measure by CCA assay .56

Figure 14 Amounts of Triacylglerides are increased in knock out mutants of puml and $\mathrm{bmm}$...........57

Figure 15 pummelig mutant flies are obese but not larvae....................................................58

Figure 16 Mean-life time is decreased in puml $^{1}$ compared to genetically matched control flies .........58

Figure 17 puml and bmm RNA levels are increased under starvation. .........................................55

Figure 18 Mortality curve of $\mathrm{bmm}^{1}, \mathrm{puml}^{1}$ and control flies under food-deprivation .......................60

Figure 19 Puml cannot hydrolyse Triolein and does not stimulate Bmm lipase activity....................62

Figure 20 A Substrate screen identifies Puml as a potent phospholipase...................................63

Figure 21 pum ${ }^{1}$ flies mobilize lipids faster during starvation than control flies.............................64

Figure 22 Locomotor activity and metabolic rate of puml ${ }^{1}$ are similar to control flies during starvation.

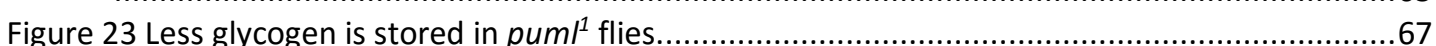

Figure 24 Desiccation resistance is decreased in puml ${ }^{1}$ flies .......................................................6 68

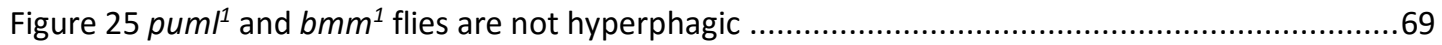

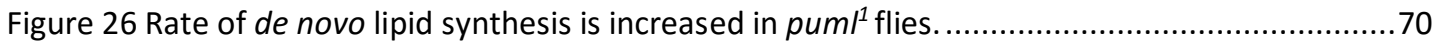

Figure 27 Puml::mCherry and Bmm::EGFP are localized to lipid droplets in adult fat body tissue......72

Figure 28 Lipid storage is drastically increased in Malpighian tubules of puml ${ }^{1}$ and $\mathrm{bmm}^{1}$ flies.........74

Figure 29 Overexpression of Puml rescues lipid over-storage phenotype in Malpighian tubules from

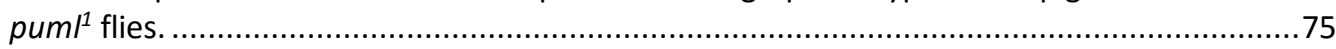

Figure 30 Puml::mCherry is localized on lipid droplets and peroxisomes .......................................76

Figure 31 Osmotic stress resistance of pum ${ }^{1}$ flies is not impaired............................................77

Figure 32 Average lipid droplet size (diameter) is reduced in Malpighian tubules of puml ${ }^{1}$ flies. .......78

Figure 33 LCFA-TAGs and abundance of PUFAs are elevated in Malpighian tubules of pum/ ${ }^{1}$ flies. ....82

Figure 34 Heat map of TAG species distribution shows increased abundance of PUFAs and shift towards longer fatty acid sidechains in Malpighian tubules from puml $l^{1}$ flies

Figure 35 A genomic rescue of puml with Puml::mCherry does not reduce the amount of body fat to control flies and does not improve ectopic lipid storage.

Figure 36 Preliminary data shows that a point mutations in each single catalytic site of Puml results in a total loss of enzymatic activity for the tested substrates of the screen. ............................89

Figure 37 Current model of pum/ regulating lipid storage in flies...............................................95

Figure 38 Preliminary results indicate that startle induced climbing activity is decreased in pum $l^{1}$ flies and a knock down of predicted peroxisomal $\beta$-oxidation genes can lead to increased body fat storage. 100

Figure 39 Preliminary data indicates that Mitochondria in Malpighian tubules from puml ${ }^{1}$ and $b m m ~^{1}$ flies are enlarged.

Figure 40 Body fat storage is unchanged in $\left.\mathrm{DmHs}\right|^{1}$ flies...................................................111

Figure 41 Neutral lipid classes are unchanged in $\left.\mathrm{DmHs}\right|^{1}$ flies................................................ 112

Figure $42 \mathrm{Hsl}:: G F P$ localizes on lipid droplets in larval fat body..........................................113 
Figure $43 \mathrm{Hsl}:$ GFP localization on lipid droplets also occurs in adult fat body tissue.

Figure $44 \mathrm{DmHs}{ }^{1}$ flies can mobilize lipids.

Figure $45 \mathrm{Hsl}:: G F P$ expressed in plin $1^{1}$ larvae and flies localizes on lipid droplets under fed and fasting conditions in larvae and adults

Figure 46 Fecundity is not impaired in $\mathrm{DmHs}^{1}$ flies ............................................................ 116

Figure 47 Preliminary results indicate that $\left.D m H_{s}\right|^{1}$ flies do not accumulate diacylglycerol. .............118

Figure 48 Cyp1::eGFP is loosely associated with lipid droplets. Cyp1::eGFP is expressed in larval fat body (FB-SNS>GAL4) of plin $1^{1}$ larvae.

Figure 49 Average lipid droplet size (diameter) is reduced in $C y p 1^{1}$ and fat body targeted Cyp1-RNAi.

Figure 50 Body fat storage is unchanged in $C y p 1^{1}$ larvae ........................................................124

Figure 51 Cyp1::eGFP expression in $C y p 1^{1}$ larvae shows a dot-like distribution in close distance to lipid droplets and non-lipid associated aggregates................................................................. 125

Figure 52 Expression of Cyp1::GFP in Cyp $1^{1}$ larvae (Lpp>GAL4) rescues small lipid droplet phenotype..

Figure 53 Overexpression of Cyp1::eGFP in plin $1^{1}$ larvae does not enhance $p$ lin $1^{1}$ giant LD phenotype..

Figure $54 \mathrm{~A}$ double knockout of $C y p 1^{1}$,plin $1^{1}$ in larvae does not prevent the giant lipid droplet phenotype of plin $1^{1}$ larvae.

\section{Index of table}

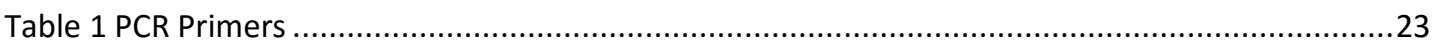

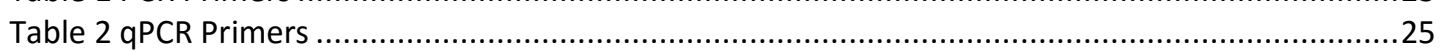

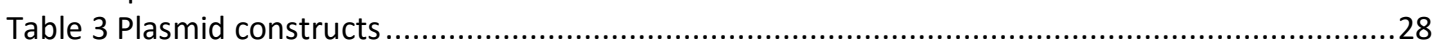

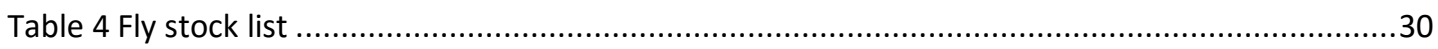

Table 5 Dyes and fluorophores used for laser scanning microscopy ............................................4

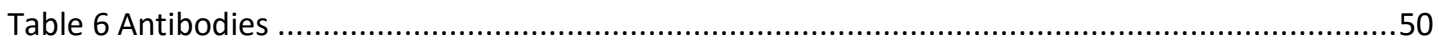

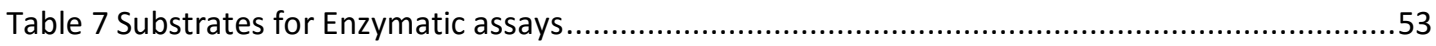




\section{Terms and abbreviations}

Akh Adipokinetic hormone

ATGL Adipose triglyceride lipase

bmm brummer

DAG Diacylglyceride

Dm Drosophila melanogaster

GFP green fluorescent protein

Hs Homo sapiens sapiens

Hsl hormone-sensitive lipase

Mm Mus musculus

NEFA non-esterified fatty acid

PA Phosphatidic acid

PC Phosphatidylcholine

PCR Polymerase chain reaction

PI Phosphatidylinositol

puml pummelig

RT room temperature

RT-qPCR Reverse transcribed quantitative Polymerase Chain reaction

SPE $\quad$ solid phase extraction

TAG Triacylglyceride 


\section{Introduction}

\subsection{Energy homeostasis}

iving systems rely on a steady influx of energy to maintain metabolic processes.

Whereas the external environment underlies a high variability, organisms try to keep a relatively constant internal energy level (Figure 1). In order to be able to adapt to the current energy needs highly ordered processes of energy uptake, storage and expenditure are taking place that combined are termed as energy homeostasis (WHO, 2014).

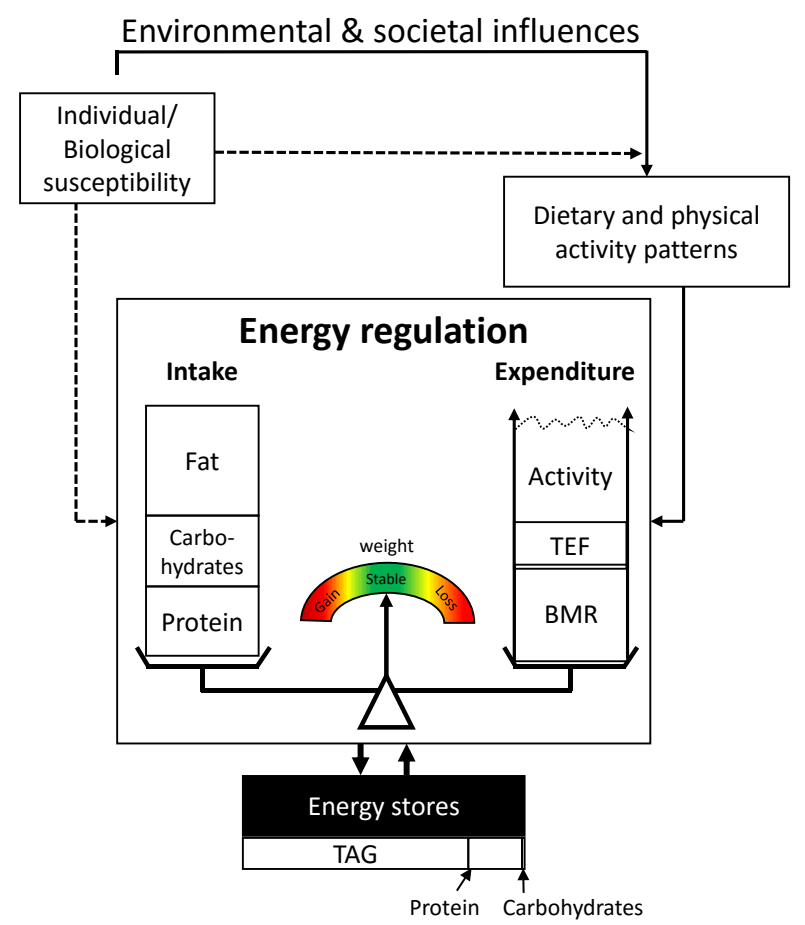

Figure 1 Factors that influence energy balance and control weight. The diagram depicts fundamental principles of energy balance and regulation. With high energy intake the energy and low energy expenditure energy balance becomes positive which promotes weight gain. The expenditure can vary greatly mostly by activity as the thermic effect of food (TEF) and basal metabolic rate (BMR) are relatively fixed parameters on the short term. It is commonly believed that the human body tends prevent undernutrition then protecting from nutrient overconsumption. However, environmental and societal influences have a great effect and may override this regulatory network. As genetic and biological factors (e.g. age and sex) cannot be control by oneself, dietary factors and physical activity provide an option to actively step into controlling energy regulation (Picture based on WHO report 894 on obesity)

Besides the basal metabolic rate energy demands of an organism can vary a lot. For instance, in resting state a 25 -year-old man $(1,80 \mathrm{~m}, 70 \mathrm{~kg})$ has a basal metabolic rate of $\sim 7.400 \mathrm{KJ} / 1761 \mathrm{cal}$ (Harris and Benedict, 1918) per day but metabolic rate can go 
up to $25.000 \mathrm{KJ}$ during strenuous exercise (Berg et al., 2007). Being chemotrophic organisms, animals rely on the uptake of nutrients to generate chemical energy by oxidation. As nutrient uptake is not always possible because of a discontinuous feeding behavior or maybe a specific developmental stage e.g. metamorphosis in insects or embryogenesis in chicken eggs, organisms developed systems to store energy.

Adenosine triphosphate (ATP) represents the universal currency of free enthalpy in biological systems (Berg et al., 2007). However, despite its key role in metabolism an average human stores only 100g ATP. Moreover, already in the resting state ( 7400KJ per day) the body uses up $40 \mathrm{~kg}$ of this molecule. Therefore, ATP needs to be regenerated continuously with energy from higher biomolecules (Berg et al., 2007).

For this, humans store and mobilize three major macromolecules namely proteins, carbohydrates and lipids. Compared to carbohydrates and proteins, lipids can store up $\sim 2,18 \mathrm{x}$ more energy per gram $(38 \mathrm{KJ} / \mathrm{g})$. Additionally, the carbon atoms in lipids have a higher reductive state compared to carbon atoms in sugars so that more energy can be generated by oxidation lipids than sugars. Neutral lipids are nonpolar and self-organizing in order to lower surface tension and therefore lower entropy. This allows a high volumetric energy storage of lipids which can save 6.75 fold more energy per gram than hydrated glycogen. Last but not least lipids are relatively inert biomolecules. Taken together lipids provide an optimal form for carbon storage in cells and might be the reason why lipids are the dominant energy storage in humans. They constitute around $82.1 \%$ of the body energy stores, followed by proteins $(17,4 \%)$, glycogen $(0.42 \%)$ and glucose $(0.08 \%)$ (Berg et al., 2007). Lipid storage is a common theme to save energy as it can be found also in nematodes, insects (e.g. fat body tissue of Drosophila melanogaster), yeast, fungi but also plants (e.g. rapeseed or sunflowers). In higher animals specialized tissues exist for lipid storage e.g. white adipose tissue in mammals or fat body in insects like Drosophila melanogaster.

Energy storage underlies a complex regulation that is partially determined by genetic predisposition but also exogenous and endogenous factors. However, a chronic 
imbalance of energy intake and expenditure can lead to a disruption of this system leading in the combination of a sedentary lifestyle and energy rich diet to obesity.

Obesity is defined as an excess storage of body fat that health may adversely effected. A crude but easy and relatively reliable categorization to classify if a person is obese is the body-mass-index (BMI) (mass $\left.[\mathrm{kg}] /(\text { height }[\mathrm{m}])^{2}\right)$. The BMI has different cut-off points. Normal weight is defined by a BMI between 18-25, followed by overweight (25-30) and obesity (BMI>30) (WHO, 2014). Obesity belongs to the noncommunicable diseases (NCDs) that became the leading cause of death in the recent years. In $2012 \sim 68 \%$ of worldwide deaths (56 Mio.) were caused by NCDs, with more than $40 \%$ of deaths were premature ( $<70$ years of age) (WHO, 2014). Obesity is increasing worldwide in both industrialized and developing countries. It even coexists with undernutrition in developing countries.

When it comes to gender specific prevalence woman a more prone to obesity than man, although rates of overweight men are higher (WHO, 2013). Another problem is a skewing in the BMI distribution that leads besides the average increase in mean population BMI to an even higher number of people with a high BMI (WHO, 2014). Besides its multifactorial elicitors obesity is associated with numerous detrimental health effects. Most importantly obesity contributes to chronic diseases like cardiovascular diseases (\#3 leading cause of death) and hypertension, cancer (\#2 leading cause for death) or diabetes but can have also less severe effects (WHO, 2014, WHO, 2016). As NCDs become more and more prominent also their consequences will have a bigger effect on the population (e.g. economic performance, financial costs of healthcare system). Therefore, it is necessary to understand the underlying regulatory networks of metabolism to fight these diseases.

In the recent years, big efforts have been undertaken to provide a better insight into metabolic regulation. Various animal model systems from human cell culture systems, over mice, nematodes ( $C$. elegans), yeast ( $S$. cerevisiae) to insects (most importantly Drosophila melanogaster) have been utilized to unravel the regulators of metabolism. This work will concentrate on lipid metabolism and will use Drosophila 
melanogaster as a model system to analyze a few mostly uncharacterized modulators of the lipid metabolism in flies.

\subsection{Lipid storage regulation in mammals}

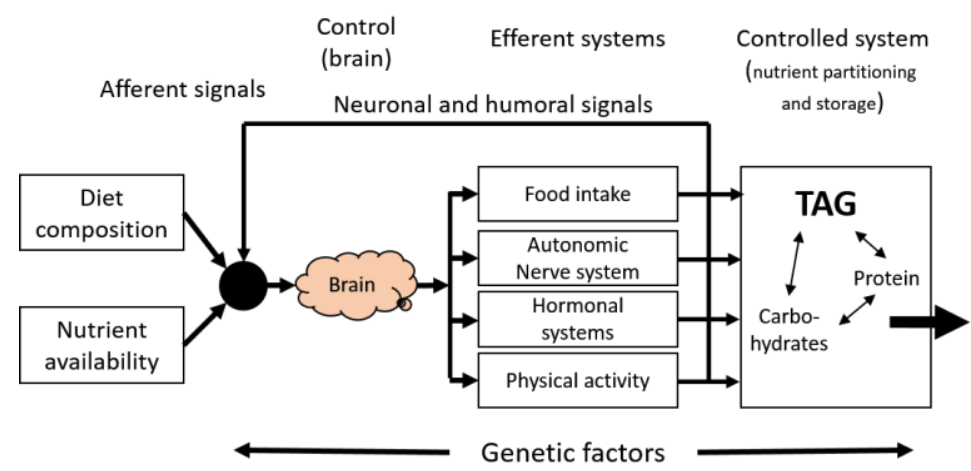

Figure 2 Physiological systems that regulates energy stores. The brain detects various afferent signals (nutrient state, diet, metabolites, hormones and neuronal) and generates a response to adapt food intake, physical activity and signalling (neuronal and hormonal) to the current needs. Additional, the system directs the nutrient partitioning to a certain set point to ensure optimal energy availability. (Scheme bases on WHO report 894 on obesity)

Although the model in (Figure 1) implies a very simple cause for obesity (ENERGY INTAKE > energy expenditure) the physiological system that actually controls energy balance is quite complex (Figure 2). It includes various signalling molecules that enable the communication between various organs: the central nervous system, adipose tissue, liver, pancreas, muscles and the digestive tract (Friedman, 2004, Speakman, 2004, Cerk et al., 2014).

As mentioned earlier the main energy storage in humans are lipids. Most in form of the neutral lipids: triacylglycerides (TAGs) in specialized cells the white adipose tissue. However, TAGs are not stored freely in the cytoplasm. Due to their non-polar character TAGs are packaged in a cellular organelle called lipid droplets. Together with other highly lipophilic substances like sterol esters, TAGs form the core of lipid droplets (LDs). This core is surrounded by a phospholipid monolayer that acts as an interphase border between the hydrophobic lipids and the aqueous cytosol (Figure 3). Furthermore, the phospholipid monolayer provides as a platform for specific proteins and serves as an intracellular compartment border to manage fundamental functions of lipid homeostasis like lipid mobilization. 


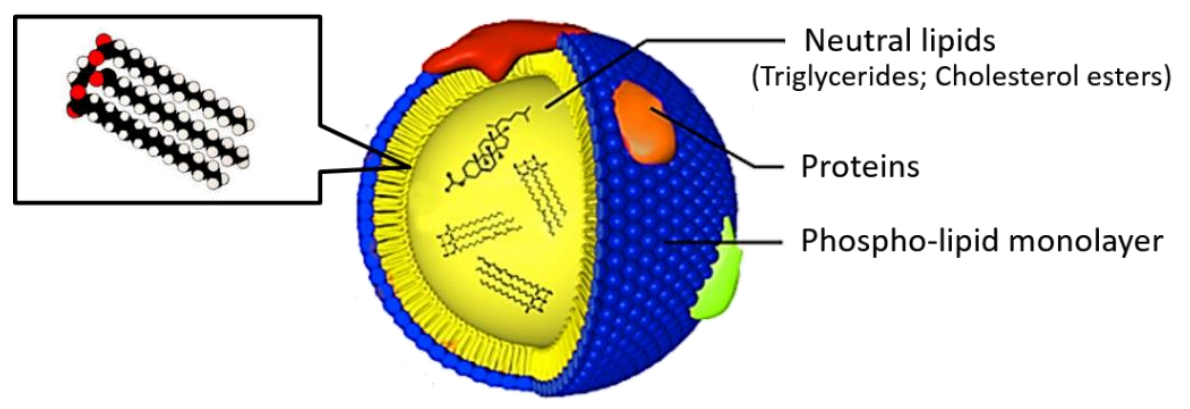

Figure 3 Schematic illustration of the lipid droplet structure. An inner core of neutral lipids (triglycerides, cholesterol esters of ceramides) is surrounded by a phospholipid monolayer (polar head groups are shown in blue). Lipid droplets can be populated by specific proteins that can be found on the phospholipid monolayer. The left structure is a model for Triolein - three oleic acid residues are esterified with a glycerol molecule. (picture modified after(Kühnlein, 2011))

\subsubsection{Lipolysis and $\beta$-oxidation}

Lipid mobilization is a well-orchestrated processes that mobilizes lipids from its storage form TAG to other lipids that can be used for membrane synthesis, act as signalling molecules or are utilized for oxidative phosphorylation to generate ATP.

In mammals, catecholamines, natriuretic peptides and insulin are considered to represent the major regulators of lipolysis in humans (Lafontan and Langin, 2009). The activation via the $\beta$-adrenergic receptor by catecholamines has been studied extensively (Granneman, 2015, Heier et al., 2016). By binding of e.g. adrenaline this G protein-coupled seven-transmembrane domain receptor (7TM GPCR) becomes activated and leads to the generation of cyclic-adenosine monophosphate (cAMP) by Adenylyl cyclase. cAMP activates Protein Kinase A (PKA) that phosphorylates and activates several enzymes involved in lipolysis (Figure 4) (Berg et al., 2007).

Perilipin1 (PLIN1) a central modulator of lipid storage is localized on lipid droplets under basal conditions and binds to $\alpha / \beta$-hydrolase domain containing 5 (ABHD5 / CGI-58). Upon PKA activation both proteins are phosphorylated by PKA (reviewed in (Londos et al., 1999). This leads to the dissociation of ABHD5 to the cytoplasm that there binds to the adipocyte triglyceride lipase (ATGL) and the complex localizes on lipid droplets again (Granneman et al., 2009). In vitro experiments showed, that this interaction is already sufficient to perform the first step in the mobilization of TAGs, the hydrolysis to Diacylglycerides (DAGs) and non-esterified fatty acids (NEFAs) (reviewed in (Oberer et al., 2011). At the same time ABHD5 stimulates through an up to now unknown mechanism the activity of ATGL (Lass et al., 2006). 
Additionally, ABHD5 binds to fatty acids binding protein (FABP) (Boeszoermenyi et al., 2015). This enhances the Triacylglyceride hydrolase (TGH) activity of ATGL even more and provides a NEFA acceptor. Moreover, it has been shown that also ATGL is a phosphorylation target of PKA (Ser ${ }^{406}$ ) and that phosphorylation increases ATGL activity as well (Pagnon et al., 2012). Antagonistically, phosphorylation of ATGL by AMPK lowers the activity of ATGL (Kim et al., 2016).

The next step in lipolysis is the hydrolysis of DAG to Monoacylglyceride (MAG) which is performed by hormone-sensitive lipase (HSL) (Fredrikson et al., 1981, Haemmerle et al., 2002a). Under basal conditions HSL is localized in the cytoplasm but after phosphorylation it trans-locates to lipid droplets and interacts with the activated and now free (not bound to ABHD5) PLIN1 (Tansey et al., 2003). Also HSL has been described to interact with FABP (Jenkins-Kruchten et al., 2003). In the last step of lipid mobilization MAGs are degraded to free glycerol and NEFA by Monacylglycerol-lipase (MAGL). The released glycerol is then transported to the liver and metabolised to pyruvate or used for gluconeogenesis (Berg et al., 2007). The NEFAs now can be used for re-esterification or are utilized for oxidative phosphorylation.

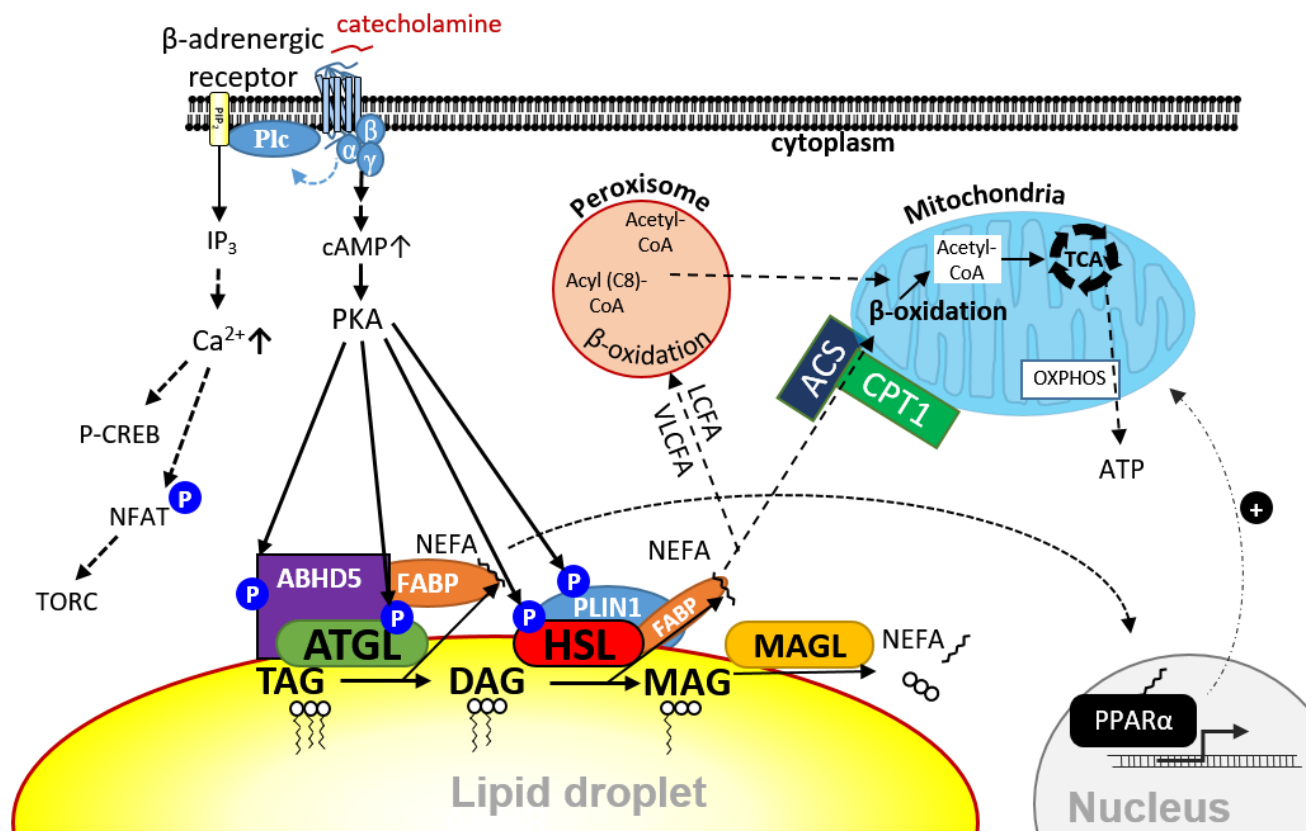

Figure 4 Schematic overview of mammalian lipid mobilization for ATP regeneration. $\beta$-adrenergic signalling leads to elevated CAMP levels that activate PKA. Phosphorylation of PLIN1 leads to the release of $A B H D 5$ that can interact with $A T G L$ and FABP to catalyse the first step in lipid mobilization the hydrolysis of TAGs. PLIN1 and phosphorylated HSL can now interact that leads to trans-location of HSL the main DAG lipase from the cytoplasm to lipid droplets. In the last step the Monoacylglycerol 
(MAG) generated by HSL is hydrolysed by MAGL. The released fatty acids from storage lipid mobilization are activated by Acyl/CoA-synthetase (ACS) and subsequently broken down in peroxisomes and mitochondria. All longer NEFAs (>C8) are transported into mitochondria via the carnitine-shuttle. The rate limiting step in the transport is catalysed by Acyl(palmitoyl)-transferase I (CPTI). The end-product of $\beta$-oxidation Acetyl-COA can be utilized in the tri-carbonic acid cycle (TCA) to produce electron donors for oxidative phosphorylation (OXPHOS) to finally generate ATP. Peroxisome proliferator-activated receptor $\alpha$ (PPAR $\alpha$ ) can sense NEFAs generated by ATGL and improve cellular substrate oxidation and respiration. Alternatively, this can be simulated by activated $p$-cAMP responsive element binding protein (CREB) or nuclear factor of activated T-cells (NFAT).

For the later NEFAs need to be activated, a process that actually needs energy in form of ATP. At the outer membrane of mitochondria, the NEFAs are bound to Coenzyme A by Acyl-CoA-synthetase (ACS) under the consumption of ATP. This two-step reaction is coupled with inorganic pyro-phosphatase cleaving the liberated pyrophosphate from the ATP into two separate phosphate ions consuming one molecule of water (Berg et al., 2007). This shifts the reaction of NEFA to Acyl-CoA towards it end-product and makes it irreversible. Subsequently, Acyl-CoA is transported into the mitochondrial matrix by utilizing carnitine-shuttle. The activated NEFAs are conjugated to the zwitterion carnitine by carnitine acyl(palmitoyl)transferase I (CPT1) that is located on the outer mitochondrial membrane as well. After that the acylated carnitine is shuttled to the inner mitochondrial membrane by a translocase by a simultaneous transport of one carnitine molecule to the outer side again. Arrived at the inner site the acyl carnitine is de-acylated by CPT2 (Berg et al., 2007). Especially, the acylation of carnitine by CPT1 is rate limiting and tightly regulated as CPT1 is inhibited allosterically by Malonyl-CoA that is generated by Acetyl-CoA carboxylase (ACC) from Acetyl-CoA. As Malonyl-CoA is an intermediate of fatty acid synthesis, high amounts inhibit beta-oxidation in mitochondria and boost lipogenesis. Particularly, medium chain fatty acids (MCFAs) depend on the Carnitine shuttle to be available for $\beta$-oxidation and subsequently oxidative phosphorylation (Figure 5). However, various ACSs exist with different acyl-chain length specificities and cellular localization (Faust et al., 2014). This mechanism is used in the hypothalamus in order to regulate food intake and glucose production (Lam et al., 2005) (Figure 5). 


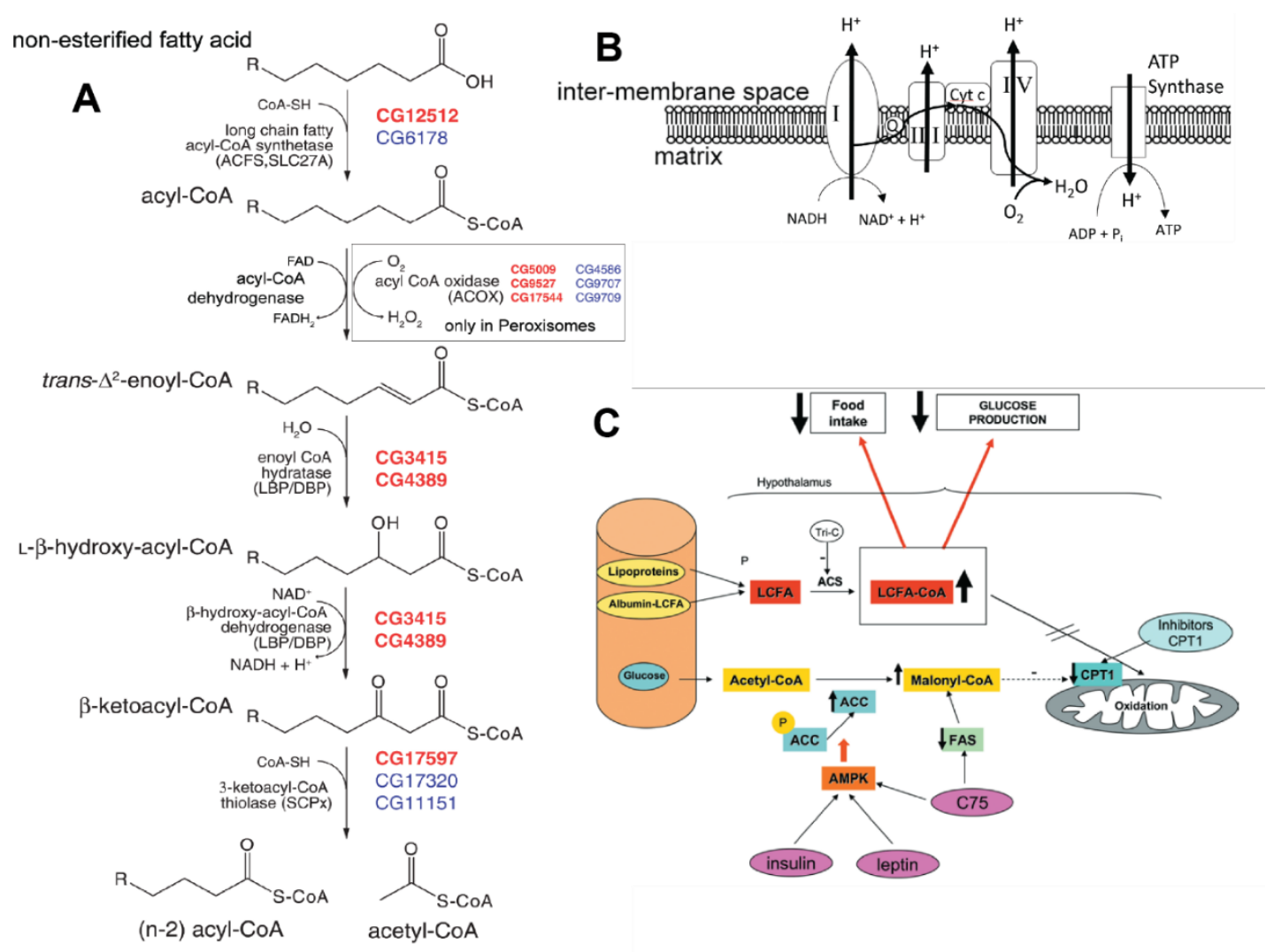

Figure 5 Schematic overview of lipid breakdown for ATP synthesis and its regulation. Chemical reactions that happen during $\beta$-oxidation in mitochondria are shown in A. Peroxisome $\beta$-oxidation only differs in the first step lipid oxidation as shown in the box in A (modified after Faust et al.2012; red (best hit) and blue are predicted Drosophila genes coding for the depicted enzyme of this reaction). An electron transport chain over $N A D H$-coenzyme $Q$ oxidoreductase (complex I), Succinate- $Q$ oxidoreductase (complex II), Q-cytochrome c oxidoreductase (complex III) and cytochrome c oxidase (complex IV) generates a proton gradient by transporting $\mathrm{H}^{+}$into the interluminal space of mitochondria. The stored energy in this gradient is used by ATP-Synthase to generate ATP (B). In the hypothalamus the generation of "activated" long-chain fatty acids (LCFA-COA) lead to signalling for decreased food intake and glucose production, shown in C. Signalling by like insulin or leptin over AMPK lead to increased Malonyl-CoA amounts that block fatty acid transport into mitochondria by blocking CPT1 that enhances LCFA-COA accumulation in the hypothalamus (picture C from (Aguilera et al., 2008))

Most of the NEFAs (90\%) are directed to mitochondria for oxidative phosphorylation (OXPHOS). However, especially very-long chain fatty acids (VLCFAs) and polyunsaturated fatty acids (acyl residues have numerous $\mathrm{C}=\mathrm{C}$ double bonds) are processed in a different cell organelle the peroxisomes. These organelles have certain characteristics. The $\beta$-oxidation in peroxisomes terminates at Octanoyl-CoA and in the first reaction of $\beta$-oxidation the dehydrogenation of Acyl-CoA the Flavoproteindehydrogenase transfers electrons to oxygen and thereby generates $\mathrm{H}_{2} \mathrm{O}_{2}$ (Figure 5). Subsequently, hydrogenperoxide is broken down to oxygen and water by catalase. In contrast, the electrons are fixed in Flavin adenine dinucleotide hydrochinone (FADH $\left.{ }_{2}\right)$ 
in mitochondria. The following steps in $\beta$-oxidation take place as in mitochondria but are performed by different isoforms of the proteins (Berg et al., 2007).

\subsubsection{Control mechanisms of lipolysis}

As described before one level of lipolysis control is the phosphorylation of several lipolytic proteins (PLIN1, ATGL, ABHD5 and HSL). The phosphorylation leads to the formation of a core module for the mobilization of TAGs consisting of at least ABHD5 and ATGL, where ABHD5 stimulates the TGH activity of ATGL. Additionally, it has been shown that ATGL changes its substrate specificity (from sn-2 to the sn-1 position of the glycerol backbone) upon activation by ABHD5, producing a more preferred substrate for HSL (Eichmann et al., 2012). Equally important is also the inhibition of ATGL by long-chain acyl-CoA (Nagy et al., 2014) and its competitive inhibitor GOS2peptide (Cerk et al., 2014). The binding to GOS2 affects the localization of ATGL to lipid droplets as well as its activity (Schweiger et al., 2012). The strategy of spatial segregation can also be applied to HSL that only recruited to lipid droplets under lipolytic conditions.

Interestingly, the released NEFAs by ATGL (but not HSL) are essential mediators for the generation of ligands for the activation of peroxisome proliferator-activated receptor alpha (PPAR $\alpha$ ). PPAR $\alpha$ activation (Figure 4) leads to improved cellular substrate oxidation and respiration (Haemmerle et al., 2011). This provides a forward loop to ensure a proper utilization of the mobilized lipids.

On the cellular level, several pathways additional modulate lipid mobilization. The energy/ATP sensor 5' AMP-activated protein kinase (AMPK) can inhibit ACC (as mentioned in the previous chapter) and therefore improving the transfer of Acyl-CoA into mitochondria (Wang et al., 2011). On the other hand, insulin signalling acts antagonistically to $\beta$-adrenergic signalling (Figure 6). The binding of insulin to the insulin receptor starts a kinase cascade that leads to the phosphorylation of the insulin-receptor substrate (IRS-1) which activates Phosphoinositid-3-kinase (PI3K) that converts the secondary messenger Phosphatidylinositol-4,5-bisphosphate ( $\left.\mathrm{PIP}_{2}\right)$ to $\mathrm{PIP}_{3}$. This again activates the $\mathrm{PIP}_{3}$ dependent protein kinase (PDK1) which phosphorylates protein kinase B (PKB / Akt) (Berg et al., 2007). 
Activated Akt then improves the glucose uptake of cells and protein synthesis by enhancing target of rapamycin (TOR) signalling, increases lipogenesis while prohibiting gluconeogenesis and lipolysis. (Figure 6).

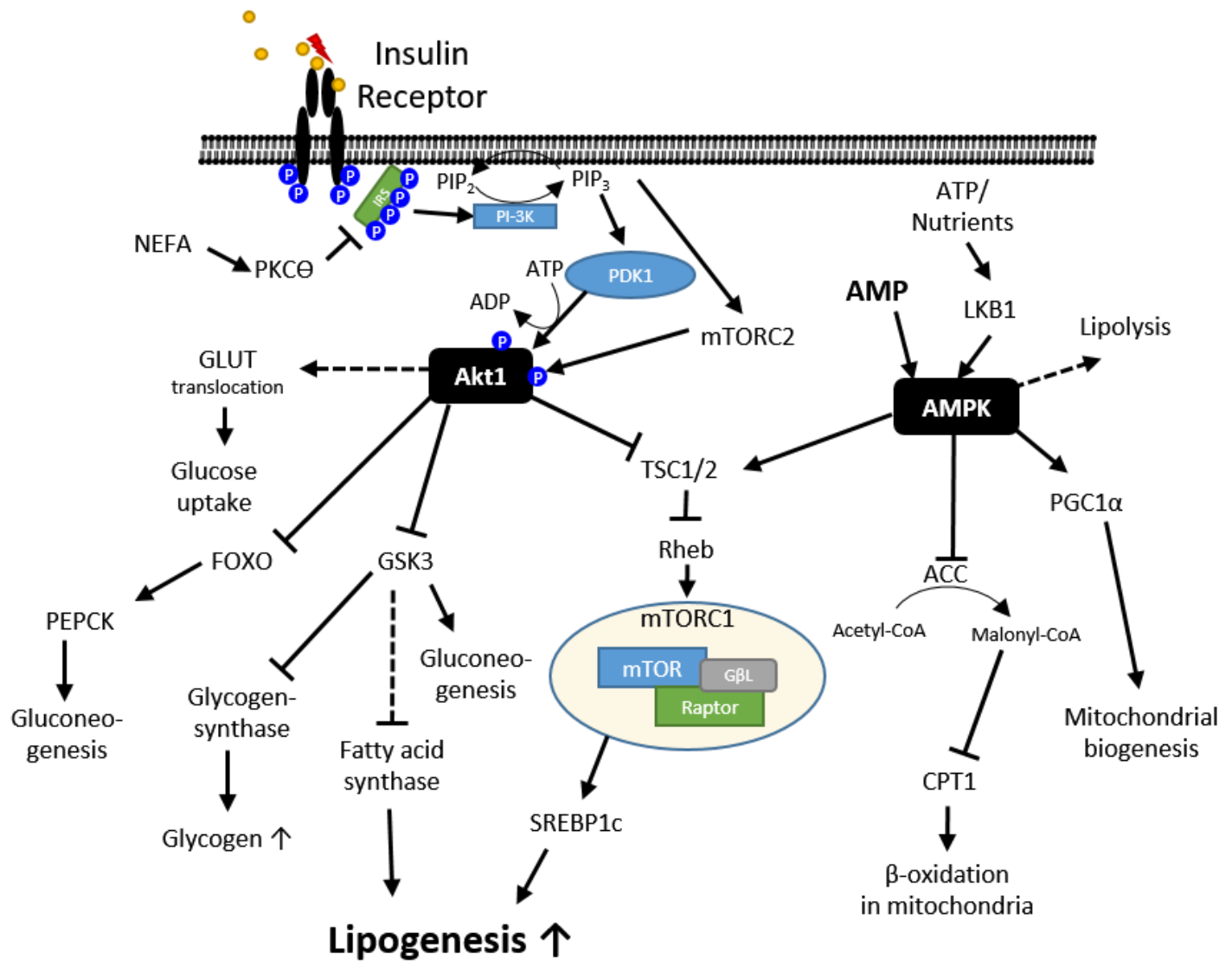

Figure 6 Schematic overview of intra-cellular energy balance regulation in mammals. Insulin signalling induces a signalling cascade that leads to increased uptake of glucose into the cells and promotes energy storage and decreased energy production by lipid breakdown in mitochondria.

\subsubsection{Neutral lipid storage disease}

Besides imbalances in energy storage caused by exogenous factors, this system is also compromised if the gene function of lipolytic genes is affected by mutations. For instance, neutral lipid-storage disease with myopathy, caused by ATGL deficiency, leads to systemic neutral lipid accumulation (Huijsman et al., 2009). Neutral lipid-storage disease with ichthyosis, also known as Chanarin-Dorfman syndrome, is a rare autosomal recessive disorder that leads to a massive lipid accumulation in various tissues and is associated with impaired function of ABHD5 (Chanarin et al., 1975). Both diseases additionally show neurological and various other phenotypes (Lefevre et al., 2001, Massa et al., 2016). However, some characteristics like ichthyosis are exclusive in ABHD5 indicating that not all functions of ATGL and ABHD5 
are limited to their interaction but that that especially ABHD5 has also ATGL independent functions (Radner et al., 2011). Also, a knockout of HSL or PLIN1 leads to lipid phenotypes (Tansey et al., 2001, Haemmerle et al., 2002b).

\subsection{Drosophila a model system for lipid research}

Insects like Drosophila melanogaster, also known as vinegar fly or fruit fly, are a very suitable model organism to study energy homeostasis. The energy homeostasis in flies is challenged by vast contrasts like the unavailability of nutrients during specific developmental stages (embryogenesis or metamorphosis) or in contrast rapid energy uptake during larval stages but also adult flies are faced with highly energy demanding situations like flight. Flies can be kept and handled easily and their small size allows breeding in large populations while being relatively inexpensive. Also, flies have a short generation time of $9-10$ days $\left(\right.$ at $25^{\circ} \mathrm{C}$ ) and a relatively small genome size ( 144 Mio. base pairs with 17700 genes distributed over 4 chromosomes) FB2015_01 (Dmel R6.04) (Attrill et al., 2016). Furthermore, Drosophila shares many sequence homologues to human diseases (Reiter et al., 2001, Chien et al., 2002, O'Kane, 2003) which makes it an ideal model organism to study these diseases.

Additionally, the Drosophila system offers a giant genomic tool box. The easy generation of transgenic flies (Spradling et al., 1999) and ectopic gene expression with the GAL4/UAS target system (Brand and Perrimon, 1993). Also site directed mutations (Crispr/Cas9 system) (Bassett et al., 2013) or imprecise P-Element excisions (Spradling et al., 1995, Spradling et al., 1999) offer tools for the generation of mutants or modification of genes.

The lifecycle of Drosophila consists of four main stages that all have different requirements on energy storage regulation: embryo, larval stages, pupae and adults (see Figure 7). As mentioned before TAGs represent the main storage form of energy. Consequently, lipids are crucial for embryogenesis, as they total rely on the mobilization of energy stores during this non-feeding developmental stage. Thus, embryos with an impairment in mobilizing lipids (Grönke et al., 2005) or generally low availability of lipids normally die (Buszczak et al., 2002, Teixeira et al., 2003). In the late stages of embryonic development a specialized tissue for lipid storage is 
formed from the mesoderm (Bate et al., 1993, Hartenstein, 1993) the Drosophila fat body that combines functions of mammalian liver and adipose tissue (Gilbert and Chino, 1974, Canavoso et al., 2001). Larvae are continuous feeders (Zinke et al., 2002) that accumulate a lot of body mass. The fat body grows up to $\sim 2500$ cells during this developmental stage and stores large amounts of TAGs that are partially mobilized during metamorphosis (Carvalho et al., 2012). Simultaneously, the larval fat body dissociates in pupae and gives rise to 800 immature adipocytes (Bodenstein, 1950) that float freely in the hemolymph in freshly eclosed flies. After 5-6 days these cells are replaced by the mature fat body tissue (Nelliot et al., 2006).
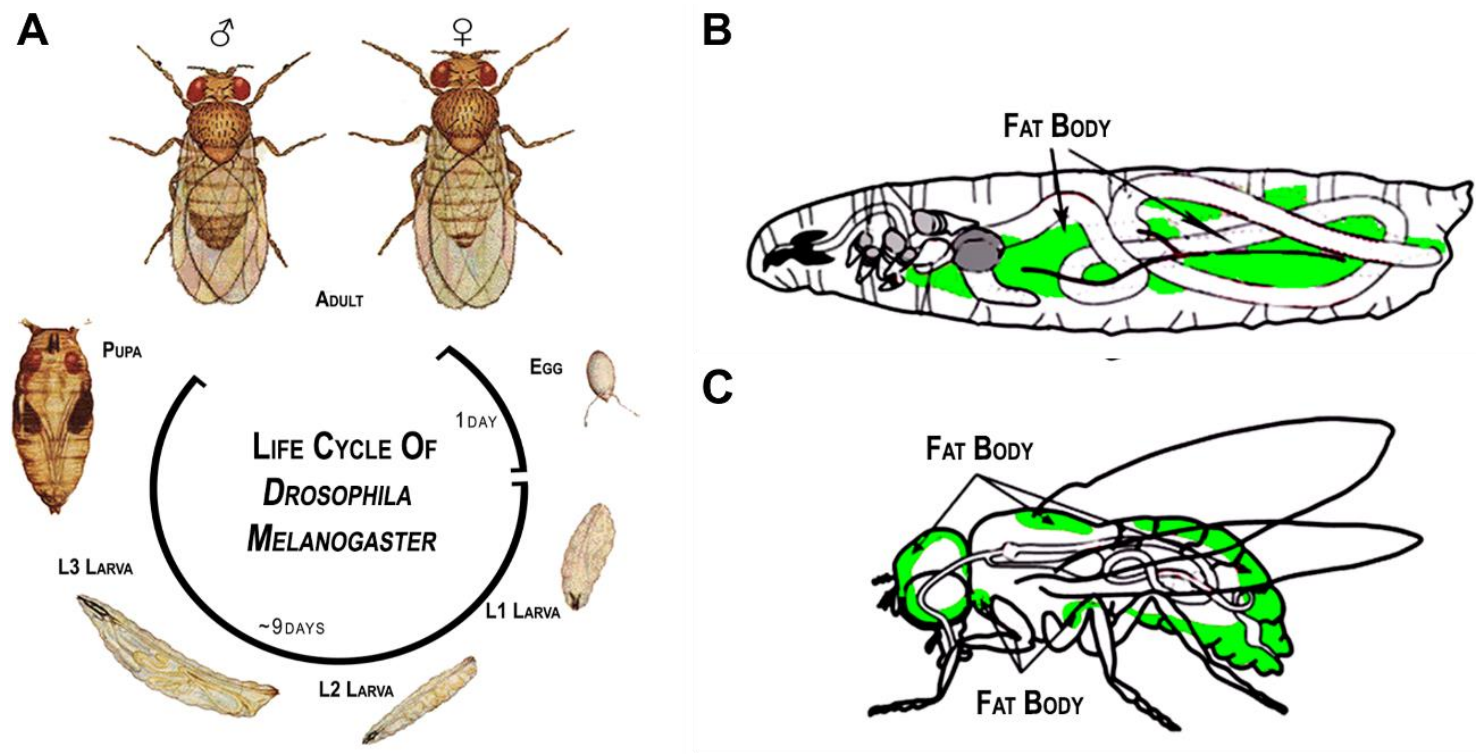

Figure 7 Life cycle of Drosophila melanogaster and distribution of the main lipid storage tissue the fat body in flies. The generation of flies is $\sim 9-10$ days at $25^{\circ} \mathrm{C}$. $1^{\text {st }}$ instar larvae hatch after $\sim 24 \mathrm{~h}$ from the fertilized egg. Several larval stages characterized by excessive accumulation of body mass and continuous feeding follow the hatching. 5 days after the start of embryogenesis the larvae pupate. After $\sim .5$ days of metamorphosis the immature adult fly ecloses (A, Scheme modified after Carolina Biological Supply Company, 2006). The main lipid storage in larvae (B) and adult flies (C) is the fat body (pictures modified after Kuhnlein, 2011). Whereas the fat body is distributed in the whole larvae and represents the major tissue it mostly localized nearby the cuticle of the fly.

Like adult mammals, also adult flies exhibit a discontinuous feeding behavior that requires a flexible system to adapt energy storage and mobilization to the current metabolic needs. Indeed, the pathways that regulate energy storage and mobilization are evolutionary conserved between mammals and flies.

For instance, the insulin pathway described earlier (Figure 6), plays also an important role in the growth, stress resistance, reproduction, longevity and metabolism in fruit 
flies (Broughton et al., 2005, Edgar, 2006, Grandison et al., 2009). The Drosophila genome encodes for seven insulin-like peptides (Dilp1-7) which have specific spatiotemporal expression patterns (Broughton et al., 2005, Grönke, 2005). Prominently, Dilp2, 3 and 5 are expressed in the median neurosecretory cells in the adult brain of Drosophila (Broughton et al., 2005, Grönke et al., 2010). These cells are of great importance as an ablation leads increased lipid storage. Also the loss of these brain Dilps increased longevity in the presence of the parasitic gut bacterium Wolbachia sp. (Grönke et al., 2010), indicating host-bacteria interaction in flies. The final mechanism between mammalian insulin and Dilps is conserved. Dilps bind to the Insulin receptor (InR) and activate the Drosophila IRS-1 (encoded by chico) starting a kinase cascade in flies. As a result, Drosophila Akt (DAkt) becomes activated and negatively regulates the activity of transcription factor Forkhead box O (FOXO), which has been described to enhance the expression of lipolytic genes like Brummer (DmATGL) or pudgy (DmACS) (Xu et al., 2012). Knock outs of either InR, chico or dilps (e.g. dilp2 or 3) bmm showed strong accumulations in body fat (Bohni et al., 1999, Brogiolo et al., 2001, Tatar et al., 2001, Grönke, 2005). Yet, the phenomenon that an impaired insulin signalling (activated; induces lipogenesis) leads to elevated TAG storage as well seems to be counterintuitive, implicating that insulin signalling is not the only signalling pathway that regulates lipid storage in the fly.

Once stimulated by insulin, fat body cells start build up lipids and store them in lipid droplets (DiAngelo and Birnbaum, 2009). A common model for the lipogenesis is that lipid droplets originate from the endoplasmic reticulum (ER) (Ohsaki et al., 2009). The nascent TAGs accumulated in the interspace of the bilayer leaflets of the ER. With further growing the outer ER membrane becomes the phospholipid monolayer for the final lipid droplets that at a certain size buds off from the ER (Tauchi-Sato et al., 2002, Farese and Walther, 2009, Beller et al., 2010). This model is supported by the finding of integral ER-membrane proteins on lipid droplets (Horiguchi et al., 2008, Zehmer et al., 2009). There is also evidence that further lipid droplet growth happens in the cytoplasm by the direct synthesis of TAGs into lipid droplets (Kuerschner et al., 2008). A key enzyme of TAG synthesis in flies is Diacylglyceride acetyltransferase (DGAT1 encoded by $m d y$ ) that catalyses the last step in TAG synthesis by the 
acetylation of DAG. An overexpression of $m d y$ leads to obese flies whereas a deficiency has the opposite effect (Buszczak et al., 2002, Beller et al., 2010).

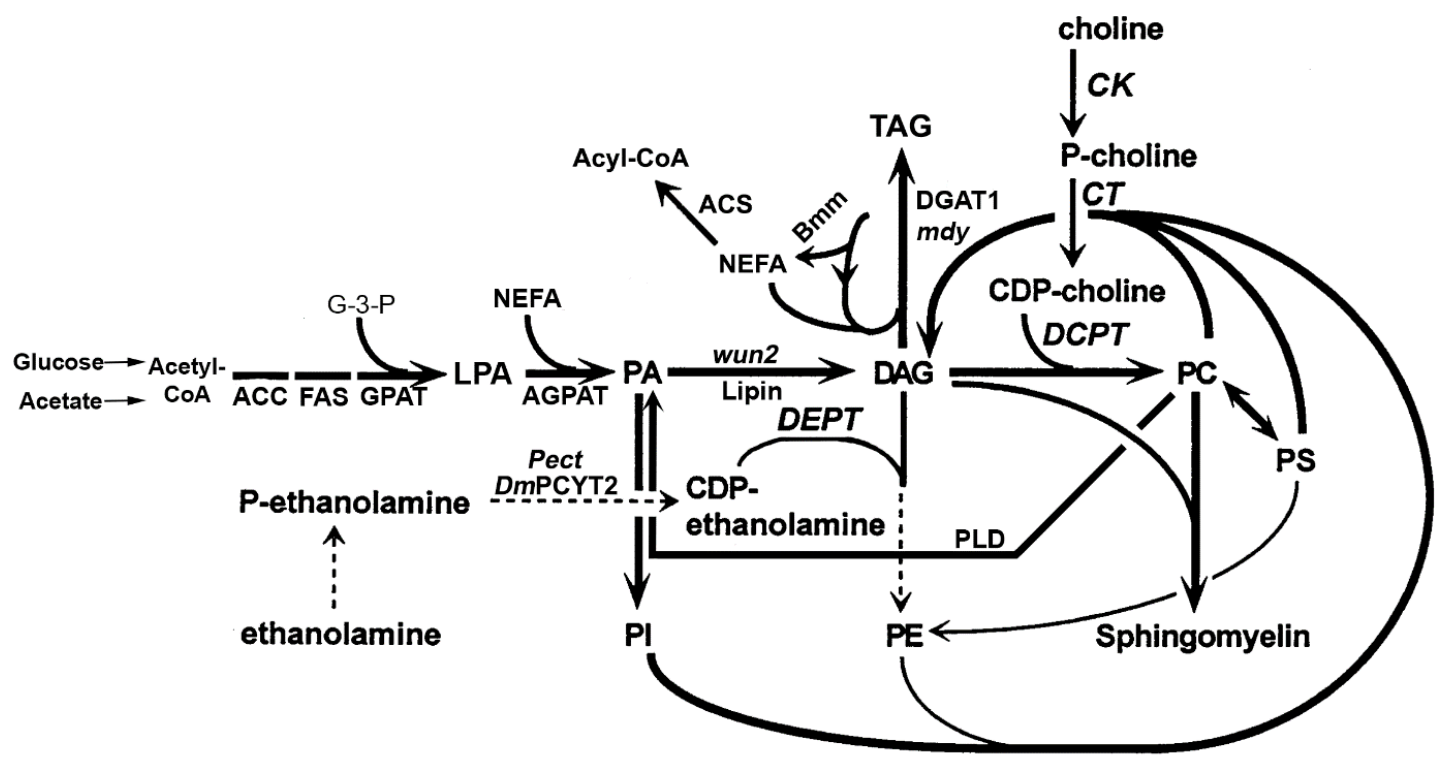

Figure 8 Schematic overview of the generation of neutral and phospholipids in Drosophila melanogaster. From Glucose of Acetate Acetyl-CoA can be generated that is used to build fatty acids which are combined with Glycerol-3-phosphate to generate Lyso-phosphatidic acid (LPA). Further incorporation of non-esterified fatty acids (NEFAs) leads to the synthesis of Phosphatidic acid (PA). PA is a precursor for phospholipids but can also be used to produce diacylglycerol (DAG) that is further acetylated to generate triacylglyceride (TAG) the main storage lipid. Alternatively, the Kennedy pathway can generate Phosphatidylethanolamine (PE) and Phosphatidylcholine (PC) with offchannelled DAG (Scheme was modified after (Igal and Coleman, 1996)).

Simultaneously with the synthesis of TAGs, also phospholipids need to be generated for the lipid droplet coat. Phospholipids are generated via the Kennedy pathway or channeled off from TAG synthesis (PA or DAG) (Figure 8). This interconnection is also emphasized by the finding that an imbalance in the phospholipid metabolism can also lead to increased amounts of TAG storage e.g. by a knock down of Pect (Figure 8) (Fullerton et al., 2009, Lim et al., 2012).

The phospholipid monolayer becomes of additional importance as it determines the size of lipid droplets (Guo et al., 2008). A critical enzyme is CTP:phosphocholine cytidylyltransferase 1 (CCT1) that is the only protein of the Kennedy pathway found on lipid droplets (Krahmer et al., 2011). CCT1 conditionally localizes on growing lipid droplets (LD) and becomes activated on the LD surface. Concomitantly with TAG synthesis also PC is generated. This phospholipid is of particular importance as it affects the size of lipid droplets most effectively (Krahmer et al., 2013). 


\subsubsection{Lipid mobilization in Drosophila melanogaster}

In order to mobilize lipids Drosophila has a system that works similar to $\beta$-adrenergic signaling in mammals, the Adipokinetic hormone (Akh)-signaling pathway (Patel et al., 2005, Grönke et al., 2007, Galikova et al., 2015). Akh is a short neuropeptide of eight amino acids that interacts specifically with the Akh receptor (AkhR) leading to lipid mobilization (Lee and Park, 2004, Galikova et al., 2015). Akh is exclusively expressed in the corpora cardiaca (a portion of the ring gland) the major neuroendocrine organ in insects (Stone et al., 1976, Noyes et al., 1995) and Akh secretion is controlled by the extracellular trehalose concentration (Rulifson et al., 2002). Interestingly, whereas Akh deficiency had no effect on ontogenesis, locomotion, oogenesis, lipid- and carbohydrate storage until the end of metamorphosis, in adults Akh regulates body fat as well as hemolymph sugar levels (Galikova et al., 2015). Comparable to a $A k h R^{1}$ mutant flies, also $A k h^{A}$ mutants have increased lipid storage but glycogen stores were normal. Consistently, both mutants have a higher starvation resistance. However, lipids were still mobilized under starvation in both mutants, indicating a second system that stimulates lipid mobilization (Grönke et al., 2007, Galikova et al., 2015).

With the binding of Akh to the AkhR in a target tissue like the Drosophila fat body it activates this GPCR leading to the activation of the $G$ protein $\alpha$ q subunit (Gaq), $G$ protein $\gamma 1$ (G 1 ) and Phospholipase $C$ at 21C (Plc21C) and subsequently increasing the intracellular $\mathrm{Ca}^{2+}\left(i \mathrm{Ca}^{2+}\right)$ and cAMP concentrations (Figure 9). An RNAi mediated knock down of $A k h R, \mathrm{G} \alpha \mathrm{q}, \mathrm{G} \gamma 1$ and Plc21C leads to decreased $i \mathrm{Ca}^{2+}$ levels (Baumbach et al., 2014b) and finally resulted in increased lipid storage in the fat body. The same effect could also be observed by a knockdown of the stromal interaction molecule (Stim) or Inositol-1,4,5,-tris-phosphate (IP3) receptor (Itpr83A/DmITPR) (Baumbach et al., 2014a).

Downstream of Akh-signalling we can find a similar pattern to mammals for the lipid mobilization (Figure 9). The activated PKA leads to the phosphorylation of DmPlin1 (Patel et al., 2005) which leads to elevated lipolytic activity (Arrese and Wells, 1994). Plin1 can be found primarily on larger "mature" lipid droplets (Beller et al., 2010) and expression as well as translation is tightly correlated with the cumulative LD surface 
area and thereby adjusts to the total fat storage in the fly (Beller et al., 2010). plin $1^{1}$ mutant flies have increased fat storage and a giant lipid droplet phenotype (LD diameter $>30 \mu \mathrm{m})$. However, plin $1^{1}$ mutant flies still can mobilize storage lipids and have a higher starvation resistance than control flies. This indicates that plin 1 is not crucial for lipid mobilization but an important member of the AkhR-dependent lipolysis pathway (Beller et al., 2010).

In Drosophila also a homolog of mammalian ATGL can be found namely Brummer (encoded by brummer or bmm). bmm knock out mutant $\left(b m m^{1}\right)$ flies are obese whereas an overexpression leads to decreased body fat storage in adult flies (Grönke et al., 2005). Like its mammalian relative that acts as the main TAG lipase in adipose tissue (Smirnova et al., 2006) also Bmm catalyses the hydrolysis of TAGs to DAGs in flies but does not processes DAGs or MAGs (Grönke et al., 2005). bmm expression correlates with the feeding state of the fly and is upregulated under nutrient deprivation (Grönke et al., 2005). Like $A k h R^{1}$ mutants also $b m m^{1}$ mutant flies are still capable to mobilize lipids during starvation. On the other hand, $A k h R^{1} \mathrm{bmm}^{1}$ double mutant as well as $b m m^{1}$ plin $1^{1}$ double mutant flies cannot mobilize lipids anymore and exhibit severe obesity (Grönke et al., 2007). 


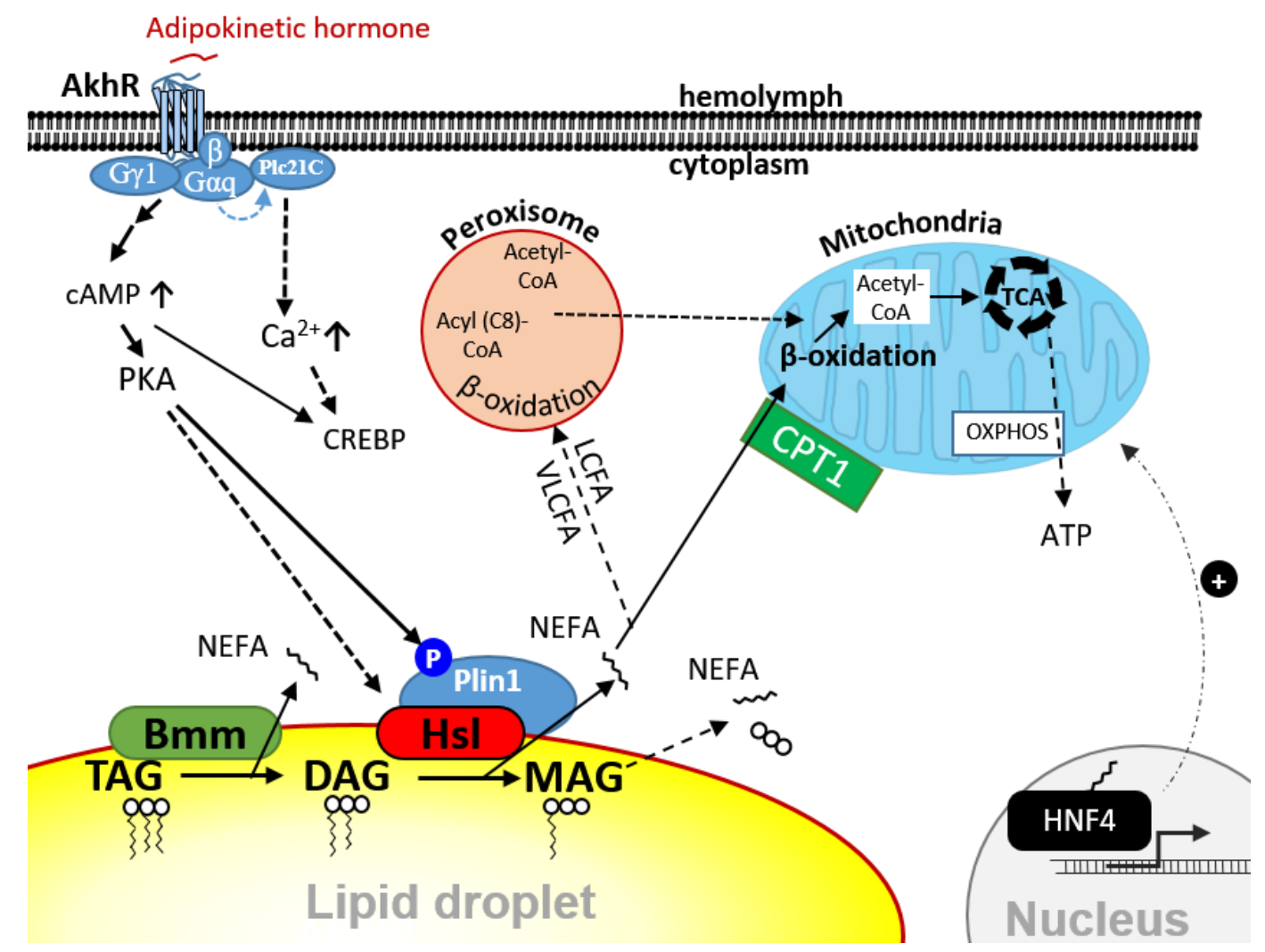

Figure 9 Schematic overview of storage lipid mobilization in Drosophila melanogaster for ATP synthesis. Adipokinetic hormone (Akh) binding to the Akh-Receptor (AkhR) induces a signal cascade that activates $P K A$ and increases intracellular $\mathrm{Ca}^{2+}$ levels and subsequently activating cAMP responsive element binding protein (CREBP). The main triglyceride lipase Brummer lipase performs TAG hydrolysis. Phosphorylated Plin1 sequesters Hsl to lipid droplets to further catalyse storage lipid hydrolysis. The released fatty acids (NEFAs) are directed towards $\beta$-oxidation in peroxisomes (especially very-long and long chain fatty acids (VLCFAs and LCFAs) and mitochondria (here imported via carnitine-shuttle with the rate limiting step catalysed by Carnitine palmitoy/transferase I (CPT1)). Acetyl-COA enters TCA and finally ATP is generated by oxidative phosphorylation (OXPHOS). Hepatic nuclear factor 4 (HNF4) can be activated by fatty acids and finally and improves cellular substrate oxidation and respiration.

However, it was not known if we can find also proteins with HSL and ABHD5 function and/or sequence similarity in the fly to see if the whole lipid mobilization pathway is evolutionary conserved. Indeed, unique sequence related proteins for HSL (Grönke, 2005) and ABHD5 (Takacs, 2007) could be found also in the fly namely Hormonesensitive lipase ortholog (encoded by Hsl, CG11055) and Pummelig (encoded by puml, CG1882). Moreover, mutants had been generated for both genes in previous works (Grönke, 2005, Takacs, 2007).

$D m H s l$ is expressed during all developmental stages with a strong enrichment in early embryogenesis indicating a strong maternal contribution (Grönke, 2005, Bi et al., 2012). $H s l^{1}$ mutant flies have normal body fat (Grönke, 2005), as so have $\mathrm{HSL}^{--}$ 
knockout mice (Haemmerle et al., 2002a). At the same time, starvation resistance of $D m H s l^{1}$ flies was not significantly different to control flies (Grönke, 2005).

Recently after I started with the further characterization of the $\left.D \mathrm{mHs}\right|^{1}$ mutants a different group published data on $\mathrm{DmHsl}$ (Bi et al., 2012).

In larvae, $\mathrm{DmHsl}$ (as well as $\mathrm{bmm}$ ) expression is upregulated during starvation (Bi et al., 2012). Bi and colleagues also generated an independent knockout mutant called $\left.D m H s\right|^{b 24}$ that was used in their experiments. The $\mathrm{DmHs}^{b 24}$ mutant larvae exhibited increased glyceride storage $(+30 \%)$ and $\mathrm{Bi}$ et al. claimed that the mutant larvae had lipid mobilization defects. At least the larval lipid over-storage phenotype was incoherent with normal fat storage of $\mathrm{DmHs}^{1}$ mutant flies. Hence, it might be possible that the over-storage phenotype is restricted to the larval stage.

A fat body specific overexpression of DmHsl::eGFP in larvae showed the same characteristics as mammalian HSL. While being localized in the cytoplasm under basal conditions, abundance of DmHsl::eGFP on lipid droplets was increased during starvation (Bi et al., 2012). Additionally, fat body overexpressed DmHsl::eGFP was unable to localize on Lipid droplets in plin $1^{1}$ larvae, leading to the assumption that, like in mammals, Plin1 sequesters DmHsl::eGFP onto the LD surface (Bi et al., 2012). However, this studies focused mainly on larvae and indicated an evolutionary conserved function of $\mathrm{DmHsl}$ to mammalian HSL (Grönke, 2005, Bi et al., 2012). Therefore, $\mathrm{DmHs}$ was analysed further to characterize its function in adult flies and identify a possible biological phenotype in $\left.D m H_{s}\right|^{1}$ flies (see Supplement).

\subsubsection{Pummelig the single sequence related protein to mammalian $\alpha / \beta$-hydrolase domain containing 4 and 5 in Drosophila melanogaster}

A BLAST research with the protein sequence from ABHD5 revealed only a single sequence related 454 amino acids long protein in Drosophila (e-value $5.58^{*} \mathrm{e}^{-93}$ ) which was named Pummelig (encoded by the gene pummelig / puml) by me. Hence, a reverse BLAST search with the Puml sequence in mammals showed also a very high similarity to mammalian ABHD4 the paralog of ABHD5. 


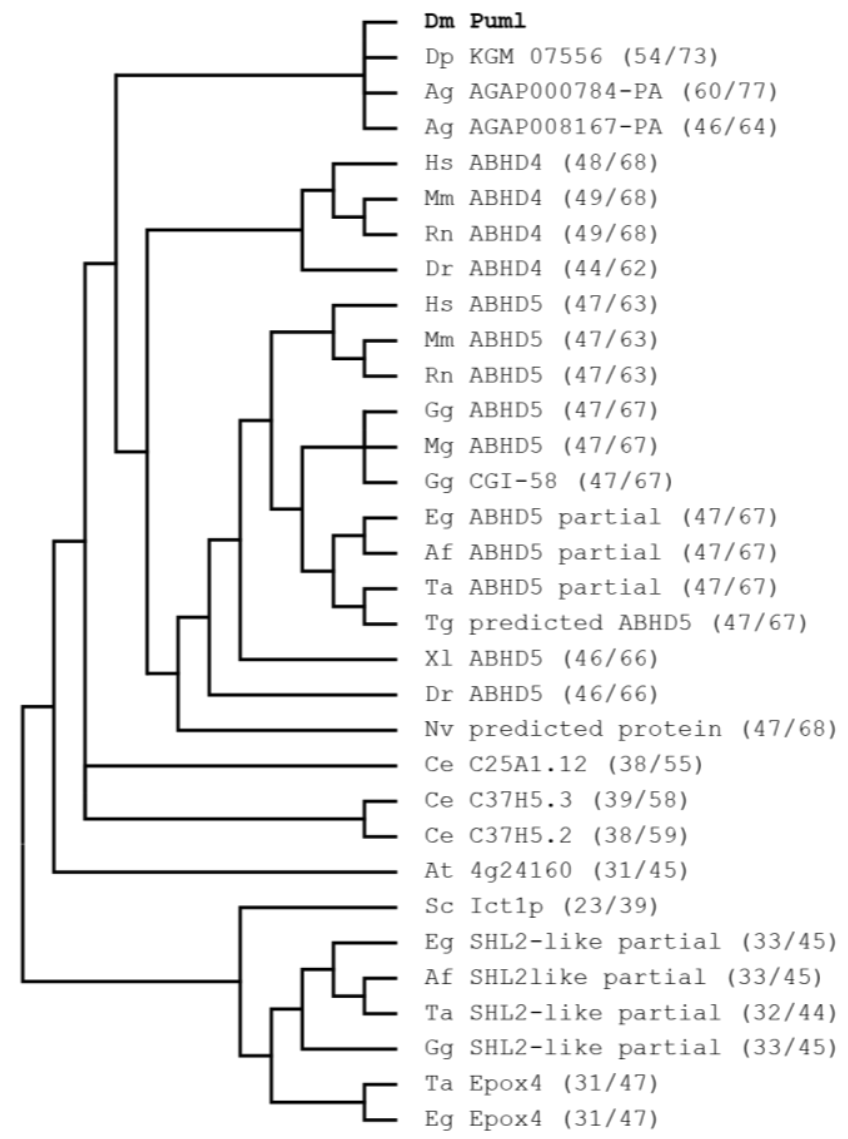

GxSxG-motif

LGHSMGGEIA

LGHSLGGYIA

LGHSMGGFIA

LGHSMGGYLA

LGHSLGGFLA

LGHSLGGELA

LGHSLGGFLA

LGHSLGGYLA

LGHNLGGFLA

LGHNLGGFLA

LGHNLGGFLA

LGHNLGGFLA

LGHNLGGFLA

LGHNLGGFLA

LGHNLGGFLA

LGHNLGGFLA

LGHNLGGFLA

LGHNLGGFLA

LGHNLGAFLA

VGHNLGGYLA

MGHSLGGFLT

IGHNFGGYLA |

VGHSFGGYLA

VGHSFGGYLS

LGHSFGGYVA

VGHSFGGYIS

MGHSMGGSVA

MGHSMGGSVA

MGHSMGGSVA

MGHSMGGSVA

IGHDWGGMIA

IGHDWGGMIA
KYSS-motif

-DIMRKFQST

-DIARKYTNY

-DIVMKFSNV

-DITSRFEGI

-DFKRKFADF

-DFKRKFADF

-DFKRKFADE

-DFKRKFEDI

-DFKRKYSSM *

-DFKRKYSSM *

-DFKRKYSSM *

-DFKRKYASM

-DFKRKYASM

-DFKRKYASM

-DFKRKYS $\mathrm{CM}$ *

-DFKRKYSSM *

-DFKRKYSSM *

-DFKRKYSSM *

-DFKKKYSSM *

-DFKKKFATM

-DFQKKYSAI

-DLLLRFPGL

-DLALKYSED

-DLEQKYSED

-GAHSEGTGL

-AYVKVPDQI

-ILLQRGATE

-ILLQRGATE

-ILLQRGATE

-ILLQRGATE

TGIGRKGCRL

TGIGRKGCRI

Figure 10 Phylogenetic analysis of Puml, conservation of the catalytic center and PKA phosphorylation site. Drosophila Puml was compared to Danaus plexippus, Anopheles gambiae, Homo sapiens, Mus musculus, Rattus norvegicus, Danio rerio, Gallu gallus, Meleagris gallopavo, Egretta garzetta, Aptenodytes forsteri, Tyto alba, Taeniopygia guttata, Xenopus laevis, Nematostella vectensis, Caenorhabditis elegans, Arabisopsis thaliana and Sacharomyces cerevisiae (left). Numbers in brackets are \%sequence identity and \% sequence similarity. A point mutation can be found in the catalytic center (GXSXG-motif) from Puml relatives in vertebrates and in C. elegans (middle). The majority of these proteins also have PKA phosphorylation site (KYSS-motif) (right). Many vertebrates with a Puml relative with an inactive catalytic center also have a second Puml-like protein with an active center (ABHD4like). The evolutionary history was inferred by using the Maximum Likelihood method based on the JTT matrix-based model. The bootstrap consensus tree inferred from 500 replicates is taken to represent the evolutionary history of the taxa analyzed. Branches corresponding to partitions reproduced in less than 50\% Bootstrap replicates are collapsed. Initial tree(s) for the heuristic search were obtained by applying the Neighbor-Joining method to a matrix of pairwise distances estimated using a JTT model. The analysis involved 32 amino acid sequences. All positions containing gaps and missing data were eliminated. There were a total of 212 positions in the final dataset. Evolutionary analyses were conducted in MEGA6.

A phylogenetic tree analysis with proteins present in: Drosophila melanogaster, Danaus plexippus, Anopheles gambiae, Homo sapiens, Mus musculus, Rattus norvegicus, Danio rerio, Gallu gallus, Meleagris gallopavo, Egretta garzetta, Aptenodytes forsteri, Tyto alba, Taeniopygia guttata, Xenopus laevis, Nematostella vectensis, Caenorhabditis elegans, Arabisopsis thaliana and Sacharomyces cerevisiae 
(Figure 10) showed that most organisms that coded for only one Puml-like protein had an active catalytic GxSxG-motif. On the other hand, in organisms with ABHD4 and ABHD5-like proteins also the PKA-target sequence "KYSS" was preferentially conserved in the ABHD5-like proteins. The closest homologue outside the Drosophilidae is Anopheles gambiae with $77 \%$ similarity. In vertebrates a clustering can be observed for the ABHD4- and ABHD5-like proteins while ABHD4-like proteins had a slightly higher similarity to Puml.

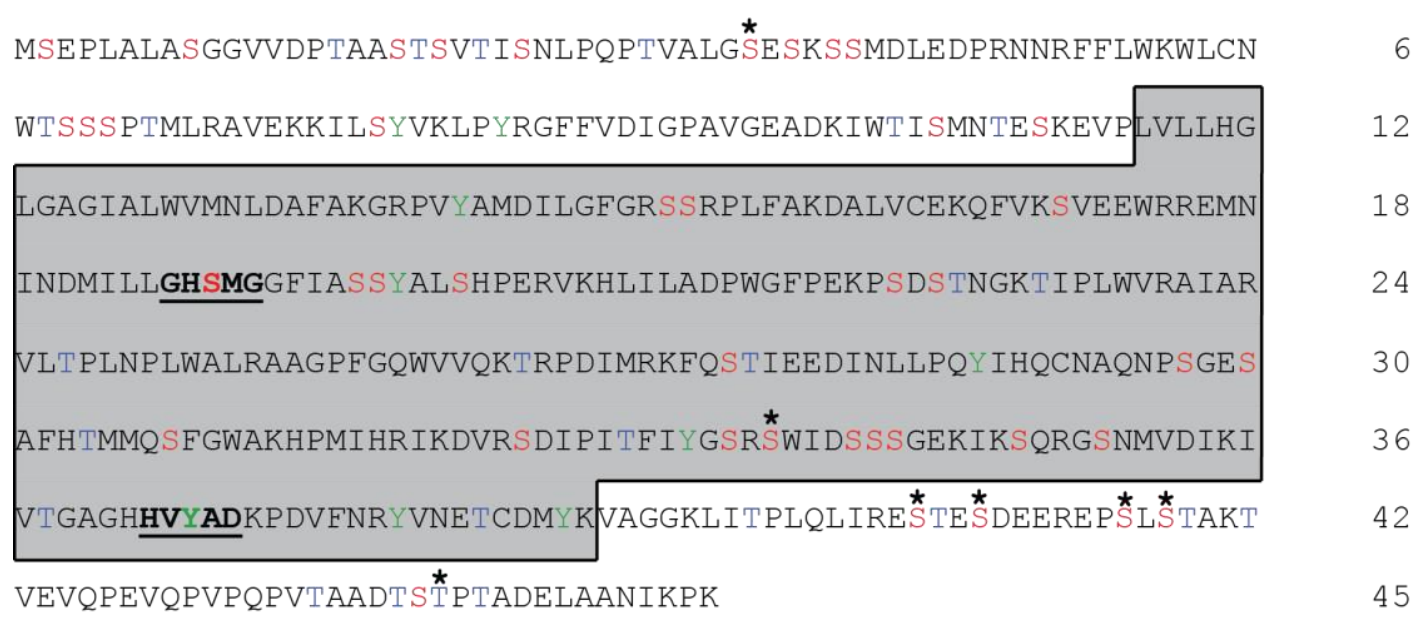

Figure 11 In silico prediction of phosphorylation sites in Puml. Analysis of Puml-PA protein sequence by DISPHOS1.3 identified 75 phosphorylation targets (serines in red, threonines in blue and tyrosine in green). The software scored six serines and 1 threonine (marked by asterisks) as potential in vivo phosphorylation sites. Most of these sites are located at the c-terminus of Puml (grey box indicates $\alpha / \beta$ hydrolase domain). The underlined amino acids represent predicted catalytic centers in Puml.

Puml belongs to the $\alpha / \beta$-hydrolase domain containing (ABHD) protein family. The core of the protein consists of eight $\beta$-sheets that are connected via $\alpha$-helices. Another characteristic are the two catalytic motifs in the serine-hydrolase motif (GxSxG) at Ser ${ }^{190}$ and an additional HxxxD-motif $\left(\mathrm{Asp}^{371}\right)$ that has been associated with Acyltransferase activity in other species (Ghosh et al., 2009, Montero-Moran et al., 2010, McMahon et al., 2014b). An in silico analysis of possible phosphorylation sites (using DISPHOS 1.3 from the Temple university, PA, USA) predicted 6 serineand 1 threonine phosphorylation sites. However, the PKA-targeted site found in ABHD5 of mammals (KYSS-motif) was not conserved in Puml (Figure 11). Also the characteristic point-mutation in the GxSxG-motif found in mammalian ABHD5 that leads to an inactive center could not be found in Puml (Figure 10). 


\subsubsection{Genomic locus of pummelig and Puml constructs}

Several tools to analyze puml function were established by previous workers on this project. A puml knock out mutant covering the protein coding site had been generated, as well as a genomic rescue construct with an c-terminal mCherry-tag (Figure 12) (Takacs, 2007). Additionally, several UAS-constructs for the expression of Puml-PA, -PB and also for both in combination with c-terminal fusions to mCherry where created beforehand (Takacs, 2007) and used to characterize puml function (Figure 12).

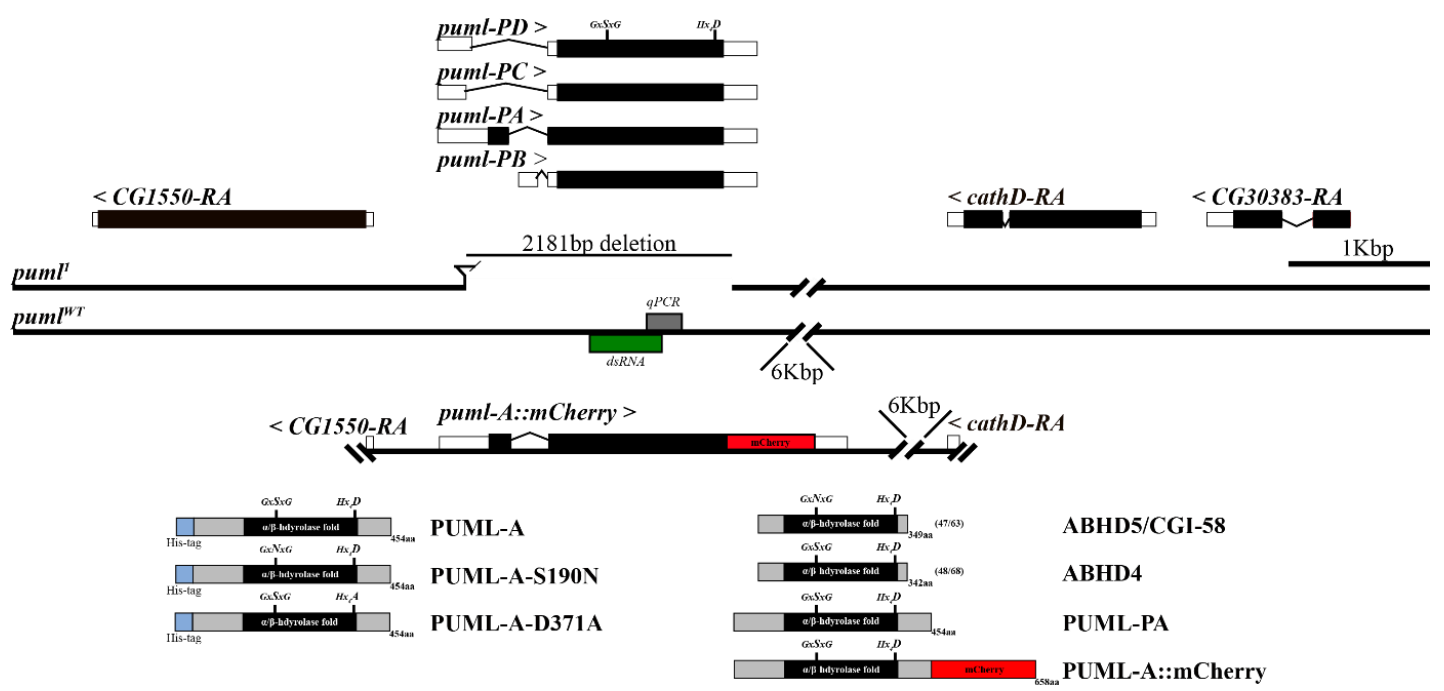

Figure 12 Genomic locus of puml, constructs and proteins available. A puml mutant had been generated that covers the protein coding sequence of puml. A genomic rescue construct was generated that covers the extended genomic region of puml and contains a c-terminal fused mCherry-tag. HisHRV3c-fused Puml variants were recombinantly expressed for enzymatic assays.

\subsubsection{Main findings from previous contributors to the characterization of pummelig}

The puml.puml-PA::mCherry transgene gene product localized on lipid droplets in larval fat body tissue as well as on LDs in adipocytes of freshly eclosed flies and exhibited a characteristic ring-localization indicating a homogenous distribution over the LD-monolayer (Takacs, 2007). The same localization behavior could be also observed if puml-PA::mCherry was overexpressed in the fat body. On the other hand, an overexpression of puml-PB::mCherry and the resulting Puml-PB::mCherry could not be found on LDs anymore but was localized punctually in the cytoplasm (Takacs, 2007). 
The pum $l^{1}$ mutant flies exhibited no difference in starvation time when compared to control flies and post mortem TAG content was insignificant from control flies (Takacs, 2007). In a later work also the TAG content of adult pum/ ${ }^{1}$ flies was quantified and showed no significant difference to control flies (Rosenberg, 2012). Also, fecundity of puml ${ }^{1}$ flies was not impaired (Rosenberg, 2012). However, a screening for lipid storage phenotypes in various tissues in puml ${ }^{1}$ larvae and adults revealed a strong increase in lipid storage in the Malpighian tubules in both developmental stages (Rosenberg, 2012).

Taken together pum ${ }^{1}$ flies exhibited no significant change in body fat but had ectopic lipid storage in the malpighian tubules. Therefore, I concentrated in this work on the further characterization of puml to identify the biological function of puml.

\section{Material and Methods}

\subsection{Molecular Biology}

\subsubsection{PCR}

Polymerase Chain reaction (PCR) enables the rapid in vitro amplification of a DNA template. For this work, PCR was used to generate amplificates for cloning or genotyping of flies and plasmids. In order to avoid the introduction of mutations into the amplificates DNA polymerases with a $3^{\prime}-5^{\prime}$ proof reading activity were used (Takara LA Taq.[Clonetech, Cat. \#: RR002A], Phusion Taq. [Thermoscientific, Cat. \#: F548L], Q5 High-Fidelity DNA Polymerase [NEB, Cat. \#: M0491S]) for PCR products that were subsequently used for cloning. For genotyping Qiagen Hot Start Master Mix (Qiagen, Cat. \#: 203443) or Phire Tissue Direct PCR 2X Master Mix (Thermo Scientific, Cat. \#: F-170L) were used. If not stated otherwise Primers were used at $0.5 \mu \mathrm{M}$ final concentration. PCR reactions were set up and run in the PCR machine according to the manufacturer recommendations of the polymerase used.

\subsubsection{Genotyping of flies using PCR}

In order to select for specific genotypes, single flies or fly batches were disintegrated and used as a template for PCR specific to the DNA sequence of interest. DNA preparation for PCR was performed after Gloor et al. (Gloor et al., 1993). In detail, 
flies were collected in 1.5mL Eppendorf tubes and snap frozen in liquid nitrogen. Then samples were smashed with a $200 \mu \mathrm{L}$ Pipette-tip containing $50 \mu \mathrm{L}$ (per fly in the vial) squishing buffer (10mM Tris-HCl pH8.2, $1 \mathrm{mM}$ EDTA, $25 \mathrm{mM} \mathrm{NaCl}$; freshly added $200 \mu \mathrm{g} / \mathrm{mL}$ Proteinase K [Sigma, Cat. \#: P6556-100MG]) in an Eppendorf tube. After smashing the buffer was expelled from the tip. Samples were then incubated for 20 $\underline{30 \mathrm{~min}}$ at room temperature. Proteinase $\mathrm{K}$ was inactivated by heating to $95^{\circ} \mathrm{C}$ for $2 \mathrm{~min}$ in a water bath. $1 \mu \mathrm{L}$ of the crude fly DNA extract was used for a PCR reaction (15$20 \mu \mathrm{L}$ recommended as minimal PCR reaction volume due to inhibitory effects of the extract on the PCR reaction).

\subsubsection{Colony PCR}

In order to identify correct genotypes of cloned plasmids in transformed E.coli colony PCRs were performed. For this, single bacteria colonies were picked with a sterile pipet tip and mixed in sterile 1 XPBS in separate $200 \mu \mathrm{L}$ PCR vials (e.g. Biorad, Cat. \#: TBC-0802). $1 \mu \mathrm{L}$ was used as PCR template. Positive clones were used for culturing in $6 \mathrm{~mL}$ LB-media (with added selective antibiotics) and plasmid preparation.

\subsubsection{Primers used for PCR}

\section{Table 1 PCR Primers}

\begin{tabular}{|c|c|c|c|}
\hline $\begin{array}{c}\text { Primer } \\
\text { ID }\end{array}$ & Target & Sequences ( $5^{\prime}$ to $\left.3^{\prime}\right)$ & Reference \\
\hline PHO953 & $b m m$ & ccagcgccggcgatgaattcATGAATCTATCATTCGCTGG & this work \\
\hline PHO954 & $b m m$ & cgactagtgagctcgtcgacTTAAAAGGCTACGTCGTG & this work \\
\hline PHO955 & puml & ccagcgccggcgatgaattcATGAGCGAACCGCTAGCA & this work \\
\hline PH0956 & puml & cgactagtgagctcgtcgacTCACTTCGGTTTGATGTTCG & this work \\
\hline PHO957 & $\mathrm{DmHsl}$ & ccagcgccggcgatgaattcATGATTGACGCGGCTTCC & this work \\
\hline PHO958 & $\mathrm{DmHsl}$ & cgactagtgagctcgtcgaCTATGAAGCGGCTAGACTTG & this work \\
\hline PHO959 & MmABHD4 & ccagcgccggcgatgaattcATGGGCTGGCTCAGCTCG & this work \\
\hline PHO960 & MmABHD4 & cgactagtgagctcgtcgacTCAGTCAACTGAGTTGCAGATCTC & this work \\
\hline PHO961 & MmABHD5 & ccagcgccggcgatgaattcATGAAAGCGATGGCGGCG & this work \\
\hline PHO962 & MmABHD5 & cgactagtgagctcgtcgacTCAGTCTACTGTGTGGCAGATC & this work \\
\hline PHO963 & MmATGL & ccagcgccggcgatgaattcATGTTCCCGAGGGAGACCAAG & this work \\
\hline PHO964 & MmATGL & cgactagtgagctcgtcgacTCAGCAAGGCGGGAGGCC & this work \\
\hline PHO965 & MmHSL & ccagcgccggcgatgaattcATGGAGCCGGCCGTGGAA & this work \\
\hline PHO966 & MmHSL & cgactagtgagctcgtcgacTCAGTTCAGTGGTGCAGCAGG & this work \\
\hline PHO975 & $\begin{array}{l}\text { 14His-HRV3C- } \\
\text { pFL- } \\
\text { screening/seq. }\end{array}$ & ATCCATGAGCAAGCACCA & this work \\
\hline PHO976 & $\begin{array}{l}\text { 14His-HRV3C- } \\
\text { pFL- } \\
\text { screening/seq. }\end{array}$ & CCTCTAGTACTTCTCGACAAGC & this work \\
\hline PHO977 & puml-seq. & GGTGTGCGAGAAGCAATTTGTG & this work \\
\hline PHO978 & puml-seq. & ATATGGTGGACATCAAGATCG & this work \\
\hline
\end{tabular}




\begin{tabular}{|c|c|c|c|}
\hline PHO979 & ATGL-seq. & GCGGCATTTCAGACAACTTGC & this work \\
\hline PHO980 & ATGL-seq. & ACTGGGTACGAAACAACC & this work \\
\hline PHO981 & ABHD4-seq. & GGCACAGTTTGGGAGGATTCC & this work \\
\hline PHO982 & ABHD5-seq. & TTCTTGGCTGCCGCTTACTC & this work \\
\hline PHO983 & MmHSL-seq. & CCGTGCTATGGCCTACTATGC & this work \\
\hline PHO984 & MmHSL-seq. & GGCCCTGGTTGTTCACATCC & this work \\
\hline PHO985 & MmHSL-seq. & GGCTTACTGGGCACAGATACC & this work \\
\hline RKO312 & $\mathrm{DmHsl}$ & AAAGATCTGAGCCGCAATAGGTGGAC & R. Kühnlein \\
\hline RKO313 & $\mathrm{DmHsl}$ & AAGGTACCCTGATGAAGCGGCTAGACTTG & R. Kühnlein \\
\hline RKO729 & $\mathrm{DmHsl}$ & CCTCAAGTATTTCCAAG & R. Kühnlein \\
\hline PHO801 & DmHsl & ACTTGGCGGAATGGGCTTG & this work \\
\hline RKO504 & $\begin{array}{l}\text { puml } \\
\text { (puml[1]) }\end{array}$ & CGGCACGCAGCATAGTTGG & R. Kühnlein \\
\hline RKO509 & $\begin{array}{l}\text { puml } \\
\text { (puml[1]) }\end{array}$ & TTTCAACCCGTTTTCAACAGG & R. Kühnlein \\
\hline RKO526 & $\begin{array}{l}\text { puml } \\
\text { (puml[1]) }\end{array}$ & TGCTTTTGCTGGCTCTGC & R. Kühnlein \\
\hline SGO140 & $\mathrm{bmm}$ & TAAACACAGATGGGGATTTGGATG & Grönke, 2005 \\
\hline SGO163 & $\begin{array}{l}b m m \\
(b m m[1])\end{array}$ & TGCCCTGTGAGAAGTGTAGA & Grönke, 2005 \\
\hline SGO186 & $\begin{array}{l}b m m \\
(b m m[1])\end{array}$ & GTTACGTGCTGCCCTCTTA & Grönke, 2005 \\
\hline
\end{tabular}

\subsubsection{Reverse transcribed quantitative Polymerase Chain reaction (RT-qPCR) for gene expression analysis}

\subsubsection{RNA extraction}

RNA was extracted with Quick-RNA MircoPrep-Kit (Zymo Research; Cat. \#: R1054) according to the manufacturer's protocol. In detail: Replicates of ten flies were collected in the provided $2 \mathrm{~mL}$ collection tubes and immediately snap frozen in liquid nitrogen and stored at $-80^{\circ} \mathrm{C}$ or processed directly. For RNA extraction $600 \mu \mathrm{L}$ RNALysis-Buffer was added to the and flies were homogenized with twenty $1,4 \mathrm{~mm}$ ceramic beads (Peqlab) using a mixer mill (Retsch, MM400) at $30 \mathrm{~s}^{-1}$ for $45 \mathrm{sec}$. The lysate was centrifuged at $16000 \times \mathrm{g}$ for $1 \mathrm{~min}$ to remove cellular debris and supernatant was transferred into a new RNAse-free vial. $600 \mu \mathrm{L}$ of Ethanol (95-100\%) was added to the supernatant, mixed well and the whole mixture was then transferred to a Zymo-Spin IC Column $(2 \times 600 \mu \mathrm{L})$ and the column was centrifuged for 30s $(16000 \times \mathrm{g})$. The flow through was discarded. In the next step, $400 \mu \mathrm{L}$ RNA-Prep Buffer was added on the column, centrifuged (30s, $16000 \mathrm{xg}$ ) and the flow through was discarded. Then, 700 $\mathrm{LL}$ RNA-Wash buffer was added, centrifuged (30s, $16000 \mathrm{x}$ g) and flow through was discarded again. In the last washing step $400 \mu \mathrm{L}$ RNA-Wash 
buffer was used and centrifuged as described before. To ensure complete buffer removal the column was centrifuged for additional $2 \min (16000 \times \mathrm{g})$ and the column was transferred into a new RNAse-free tube. RNA was eluted with $30 \mu \mathrm{L}$ preheated $\left(\sim 95^{\circ} \mathrm{C}\right) \mathrm{H}_{2} \mathrm{O}$ (RNAse-free) by centrifugation (30s, $\left.16000 \times \mathrm{g}\right)$. RNA concentration was measured with a NanoDrop 1000 and used directly or stored at $-80^{\circ} \mathrm{C}$.

\subsubsection{Reverse Transcription}

Reverse Transcription and DNAse digestion was performed with the Qiagen QuantiTect Reverse Transcription Kit (Qiagen, Cat. \#: 205311) according to the manual. gDNA Wipeout buffer was warmed to room temperature before use. $1 \mu \mathrm{g}$ total RNA was used with $1 x$ gDNA Wipeout buffer (7x stock) in a final volume of $14 \mu \mathrm{L}$. The reaction was incubated for $2 \mathrm{~min}$ at $42^{\circ} \mathrm{C}$ and afterwards immediately stored on ice. Then, $4 \mu \mathrm{L}$ RT Buffer $(5 \mathrm{x}), 1 \mu \mathrm{L}$ PrimerMix and $1 \mu \mathrm{L}$ of reverse Transcriptase was added to the mix on ice. The complete mix was then incubated for $15 \mathrm{~min}$ at $42^{\circ} \mathrm{C}$ followed by $3 \mathrm{~min}$ of heat inactivation $\left(95^{\circ} \mathrm{C}\right)$. The generated cDNA was directly used or stored at $-20^{\circ} \mathrm{C}$. For the negative control and possible detection of genomic DNA contamination an additional replicate from the same total RNA was treated the same way except the addition of $1 \mu \mathrm{L}$ water instead of reverse transcriptase (-RT control).

\subsubsection{3 qPCR}

The generated cDNA was used as template for quantification of gene expression. Samples were diluted (1:25) in $\mathrm{H}_{2} \mathrm{O}_{\text {dd }}$ for the reaction. For the PCR reaction the RotorGene SYBR Green PCR Kit (Qiagen, Cat. \#: 204076) was used. 10 $\mu$ L SybrGreen-Mix were added to $2 \mu \mathrm{L}$ cDNA template. Gene-specific primers (finally $2 \mathrm{mM}$ in reaction) were added and $\mathrm{H}_{2} \mathrm{O}_{\text {dd }}$ was added to a total reaction volume of $20 \mu \mathrm{L}$.

Following primers were used:

Table 2 qPCR Primers

\begin{tabular}{|ccccc|}
\hline Symbol & Gene & Efficiency & $\begin{array}{c}\text { Amplicon } \\
\text { size / bp }\end{array}$ & Ordering \#/ Sequence \\
\hline RpL32 & $\begin{array}{c}\text { Ribosomal } \\
\text { protein L32 }\end{array}$ & $\begin{array}{c}\sim 100 \% \\
\text { (Qiagen) }\end{array}$ & 115 & QT00985677 \\
\hline Act5c & Act5c & $\begin{array}{c}100 \% \\
\text { (Bauer } \text { et } \\
\text { al., 2009) }\end{array}$ & 151 & $\begin{array}{c}5^{\prime} \text { GTGCACCGCAAGTGCTTCTAA 3 } \\
5^{`} \text { TGCTGCACTCCAAACTTCCAC 3` }\end{array}$ \\
\hline
\end{tabular}




\begin{tabular}{|c|c|c|c|c|}
\hline EF1 & $\begin{array}{c}\text { Elongation Factor } \\
1 \text { alpha100; } \mathrm{Tm} \\
\sim 60^{\circ} \mathrm{C}\end{array}$ & $\begin{array}{c}106 \% \\
\text { (Ponton et } \\
\text { al., 2011) }\end{array}$ & 125 & $\begin{array}{l}\text { 5' GCGTGGGTTTGTGATCAGTT 3' } \\
\text { 5'GATCTTCTCCTTGCCCATCC 3' }\end{array}$ \\
\hline bmm & $\begin{array}{l}\text { brummer } \\
\text { (CG5295) } \\
\end{array}$ & $100 \%$ & 104 & QT00964460 \\
\hline puml & $\begin{array}{l}\text { pummelig } \\
\text { (CG1882) }\end{array}$ & $97 \%$ & 68 & QT00941493 \\
\hline$H s l$ & $\begin{array}{c}\text { DmHsl } \\
\text { (CG11055) }\end{array}$ & 100\% & 78 & PPD05253A-200 \\
\hline
\end{tabular}

Amplification cycles and analysis was performed on a Qiagen Rotor-Gene Q System. Technical triplicates were run for each tested cDNA sample and Primer combination. Reactions were performed in 0.1mL tube stripes (Qiagen, Cat. \#: 981103). Following standard program was used: $5 \mathrm{~min}$ at $95^{\circ} \mathrm{C}$ followed by 40 amplification cycles ( $5 \mathrm{sec}$ at $95^{\circ} \mathrm{C} \rightarrow 10 \mathrm{sec}$ at $\left.60^{\circ} \mathrm{C}\right)$. Finally, a melting curve was generated $\left(65-95^{\circ} \mathrm{C} ; 1^{\circ} \mathrm{C}\right.$-steps; $5 \mathrm{sec}$ duration of each step). Cycle of quantification (Cq) was determined in the RotorGene Q Software. Further calculations were performed using Microsoft Excel 2013. Relative gene expression levels were calculated with $\Delta \Delta^{\mathrm{Cq}}$ method (Pfaffl et al., 2002) using two reference genes (chosen after(Ponton et al., 2011)).

\subsubsection{Restriction of DNA}

For cloning and size analysis DNA templates were subjected to a restriction digest with restriction endonucleases (type II). For size analysis $\sim 1 \mu \mathrm{g}$ DNA template was incubated for $1 \mathrm{~h}$ with $2 \mathrm{U}$ of a selected restriction enzyme in a reaction volume of $20 \mu \mathrm{L}$ (buffer and incubation temperature according to the manufacturer).

DNA fragments were separated by gel electrophoresis in a 1XTBE-Buffer system (90mM Tris-borate, 1mM EDTA; $\mathrm{pH} ~ 8.3$ ). Gel percentages were adjusted to the needed resolution range (0,5 - 2,5\% Agarose). Gels were incubated in Ethidium bromide in order to visualize nucleic acids under UV light.

\subsubsection{Gel extraction of DNA fragments}

In order to extract size separated DNA fragments from an Agarose Gel, pieces with the wanted fragment were cut out. Extraction of DNA was performed with the QIAquick Gel Extraction Kit (Qiagen, Cat. \#: 28706) according to the manufacturer protocol. 


\subsubsection{Gibson assembly cloning}

Gibson assembly was used to join overlapping DNA fragments in a single-tube isothermal reaction. In this work it was used to clone various genes into the $14 \mathrm{His}-$ HRV3C-pFL-vector for Baculovirus expression in Sf-9 and Hi-5-cells.

The needed primers were designed with the NEBuilder Assembly Tool (http://nebuilder.neb.com/). PCR reaction was performed with a high fidelity polymerase as mentioned above. For all constructs the same touch-down PCR program was used: $\left(1 \times 95^{\circ} \mathrm{C}\right.$ for $3 \mathrm{~min}$ followed by a cycle of $95^{\circ} \mathrm{C}$ for $30 \mathrm{~s} \rightarrow$ step-wisegradient from $62-54^{\circ} \mathrm{C}\left(-1^{\circ} \mathrm{C} / \mathrm{cycle}\right)$ for $30 \mathrm{~s} \rightarrow 72^{\circ} \mathrm{C}$ for $3 \mathrm{~min}$. Then followed by 10 cycles with the lowest annealing temperature and a final amplification step with $72^{\circ} \mathrm{C}$ for $10 \mathrm{~min}$. Total reaction volume was $50 \mu \mathrm{L}$. As templates existing plasmids or according CDNA was used for the wanted genes.

PCR amplificates were separated by gel electrophoreses and extracted from the gel. The 14His-HRV3C-pFL vector (gift from (Trowitzsch et al., 2010)) was linearized using EcoRI (NEB), separated on an Agarose gel and extracted from the gel. Gibson assembly was performed according to the manufacturer protocol. The assembled DNA template was then transformed into bacteria and screened for positive integrations. Positively confirmed clones (via colony PCR) were cultured and subjected to a plasmid preparation (midi-prep). Final vectors (14His-HRV3C-GOI-pFL) were analysed by a restriction digest and DNA-fragment size analysis. Vectors with expected fragment sizes were kept and sequenced in order to ensure correct DNA sequences for a successful expression.

\subsubsection{Transformation of E.coli}

\subsubsection{Transformation with chemically competent cells}

$50 \mu \mathrm{L}$ (in a $1.5 \mathrm{~mL}$ Eppendorf tube) of chemically competent cells were thawed on ice. Desired DNA template $(2 \mu \mathrm{L})$ was added and gently mixed by flicking the tube. The mix was incubated for 30 min on ice. Afterwards a heat shock was performed for 30s! at $42^{\circ} \mathrm{C}$ (do not mix the sample). The tube was then immediately transferred on ice for $2 \mathrm{~min}$. Then $950 \mu \mathrm{L}$ of SOC-medium (pre-warmed to $37^{\circ} \mathrm{C}$ ) were added (without antibiotics) and bacteria were cultured for $1 \mathrm{~h}$ at $37^{\circ} \mathrm{C}$ under vigorous shaking. Finally, 
$100 \mu \mathrm{L}$ of the culture were spread on LB-Agar plates with the needed selective antibiotics and incubated over night at $37^{\circ} \mathrm{C}$.

\subsubsection{Transformation with electro competent cells}

$50 \mu \mathrm{L}$ of electro competent cells were thaw on ice and transferred into an electroporation cuvette (pre-chilled on ice). $2 \mu \mathrm{L}$ of DNA template were added to the cells and mixed gently by pipetting up and down. Electroporation was performed in a Biorad Electroporator (Program Ec2). After the electro pulse cells were taken up in $950 \mu \mathrm{L} \mathrm{SOC}$ and incubated for $1 \mathrm{~h}$ at $37^{\circ} \mathrm{C}$ under vigorous shaking. Finally, $100 \mu \mathrm{L}$ were distributed homogenously on a LB-Agar plate with the needed selective antibiotics and incubated over night at $37^{\circ} \mathrm{C}$.

\subsubsection{List of plasmids}

Table 3 Plasmid constructs

\begin{tabular}{|c|c|c|c|}
\hline Plasmid ID & Construct & Vector & Reference \\
\hline RK446 & Strep-Tag-DmHsl & pASK IBA5+ & C. Heier \\
\hline RK444 & 6xHis-DmHsl & pcDNA4 Hismax & C. Heier \\
\hline AH435 & PUML & pUASTattB & A. Hildebrandt \\
\hline PH486 & 6xHis-MmABHD5 & pcDNA4 Hismax & C. Heier \\
\hline $\mathrm{PH} 487$ & 6xHis-mmABHD4 & pcDNA4 Hismax & C. Heier \\
\hline $\mathrm{PH} 488$ & Bmm-GST & - & C. Heier \\
\hline PH489 & 6xHis-MmHsl & pcDNA4 Hismax & C. Heier \\
\hline $\mathrm{PH} 490$ & 14xHis-HRV3C-pFL-vector insert & pUC57 & this work \\
\hline PH491 & 14xHis-HRV3C-MmATGL & $\mathrm{pFL}$ & this work \\
\hline $\mathrm{PH} 492$ & 14xHis-HRV3C-ABHD5 & $\mathrm{pFL}$ & this work \\
\hline $\mathrm{PH} 493$ & 14xHis-HRV3C-MmHSL & $\mathrm{pFL}$ & this work \\
\hline $\mathrm{PH} 494$ & 14xHis-HRV3C-Dmbmm & $\mathrm{pFL}$ & this work \\
\hline $\mathrm{PH} 495$ & 14xHis-HRV3C-PUML & $\mathrm{pFL}$ & this work \\
\hline $\mathrm{PH} 496$ & 14xHis-HRV3C-DmPUML-S190N & $\mathrm{pFL}$ & this work \\
\hline $\mathrm{PH} 497$ & 14xHis-HRV3C-DmPUML-D371A & $\mathrm{pFL}$ & this work \\
\hline $\mathrm{PH} 498$ & 14xHis-HRV3C-DmPUML-S190N-D371A & $\mathrm{pFL}$ & this work \\
\hline $\mathrm{PH} 499$ & 14xHis-HRV3C-DmHsI & $\mathrm{pFL}$ & this work \\
\hline PH500 & pFL-Donor-vector & $\mathrm{pFL}$ & V. Pena \\
\hline PH501 & 14xHis-HRV3C-pFL & $\mathrm{pFL}$ & this work \\
\hline
\end{tabular}

\subsection{Fly husbandry}

If not stated otherwise flies were propagated on a complex corn flour-soy flourmolasses medium (corn flour and barly malt each $69,57 \mathrm{~g} / \mathrm{L}$; soy flour $8,7 \mathrm{~g} / \mathrm{L}$; molasses/beet syrup 19,13g/L; yeast $15,65 \mathrm{~g} / \mathrm{L}$; agar-agar $5,7 \mathrm{~g} / \mathrm{L}$; propionic acid $5,43 \mathrm{~mL} / \mathrm{L}$; methyl 4-hydroxybenzoate/nipagin $1,3 \mathrm{~g} / \mathrm{L}$ ) furthermore referred to as 
Göttingen food (Gö-food). Flies reared at $25^{\circ} \mathrm{C}$ ( $60 \%$ humidity; $12 / 12$ light/dark cycle) in midsize vials (Greiner, PS-Dosen, 68mL, 36/83mm Cat. \#:217101) with a few added crumbs of live-yeast and a filter paper placed in the food (Macherey-Nagel, Filterpaper folded $\varnothing 7 \mathrm{~cm}$; Cat. \#:531007). Vials were enclosed with mite proof plugs ( $\varnothing 36 \mathrm{~mm}$ Mite proof plug, K-TK). Density was only controlled for flies used for experiments.

\subsection{Genetics}

\subsubsection{Ectopic gene expression via the GAL4/UAS-System}

The GAL4/UAS-System allows the selective ectopic expression of a cloned gene in various tissue and cell-specific patterns. The two-part system consists of the GAL4 yeast transcription factor and its corresponding DNA target site the UAS-element (upstream activating sequence) (Brand and Perrimon, 1993). To achieve the spatiotemporal specificity, the Driver/GAL4-line carries a specific enhancer sequence upstream of the GAL4 gene. Fly effector lines carry downstream of the UAS-site a gene of interest. The F1 generation offspring of the Driver/Effector-interbreed expresses the GAL4 protein depending on the enhancer sequence of the Driver. As the UAS-target site is available GAL4 can now bind to it and induce gene expression of the target gene.

\subsubsection{Backcrossing}

In order to avoid inbreeding effects and random genomic modifiers leading to unwanted phenotypes within the population and getting a genetically similar control fly stock, flies were crossed with an isogenic host strains (e.g. $\mathrm{w}^{1118}$ from VDRC). Virgin females of each generation were collected and selected for allele of interest and crossed again with male flies from the isogenic host strain. Due to recombination during oogenesis, genetic variability was increased in the fly population. After $\sim 9$ generations fly lines were established again and the isogenic host strain could be used as the genetically matched control strain. 


\subsection{Fly stocks}

Table 4 Fly stock list

\begin{tabular}{|c|c|c|c|c|}
\hline Flystock \# & Genotype & Cyt. & Description & Reference \\
\hline RKF1084 & $w^{1118}$ & 1 & $\begin{array}{l}\text { Isogenic host } \\
\text { strain }\end{array}$ & $\begin{array}{c}\text { VDRC } 60000 \\
\text { (Dietzl et al., } \\
2007)\end{array}$ \\
\hline JRF1235 & $w^{1118} ;$ puml ${ }^{1}$ & 1,2 & puml mutant & $\begin{array}{c}\text { (Rosenberg, } \\
\text { 2012) }\end{array}$ \\
\hline RKF125 & $w^{*} ; P\left\{w^{+m W \cdot h s}=G a w B\right\} F B+S N S$ & 1,2 & $F B-G A L 4$ & $\begin{array}{l}\text { Grönke et al., } \\
2003\end{array}$ \\
\hline RKF182 & $\begin{array}{l}y[1] w^{*} ; P\{w[+m C]=A c t 5 C-G A L 4\} 25 F O 1 / C y O, \\
y[+]\end{array}$ & 1,2 & Act5C-GAL4 & BDSC 4414 \\
\hline SGF529 & $w^{*} ; b m m^{1} / T M 3, S b^{1}$ float. & 1,3 & $b m m^{1}$ & $\begin{array}{c}\text { (Grönke et al., } \\
\text { 2005b) }\end{array}$ \\
\hline MGF1566 & $w^{1118} ; b m m^{1} / T M 3$ Ser $^{*}$ & 1,3 & $\begin{array}{c}b_{m m^{1}} \\
\text { backcrossed into } \\
\text { RKF1084 for } 9 \\
\text { generations }\end{array}$ & M. Galikova \\
\hline RKF887 & $\begin{array}{l}w^{*} ; P\{w[+m C] \cup A S T-p u m l-P A: m C h e r r y\} \# 48 A / \\
\text { CyO-hb-beta-gal float }\end{array}$ & 1,2 & $\begin{array}{l}\text { UAS-puml- } \\
\text { PA::mCherry }\end{array}$ & (Takacs, 2007) \\
\hline RKF888 & $\begin{array}{l}w^{*} ; P\{w[+m C] \cup A S T-p u m l-P A: m C h e r r y\} \# 22 A / \\
T M 3 S b[1] \text { e[1] float }\end{array}$ & 1,3 & $\begin{array}{l}\text { UAS-puml- } \\
\text { PA::mCherry }\end{array}$ & (Takacs, 2007) \\
\hline RKF1238 & $\begin{array}{l}w^{*} ; P\{U A S T a t t B-p u m l-P A-S 190 N\} a t 86 F b \# 3 / \\
T M 3, \text { Sb[1] float }\end{array}$ & 1,3 & $\begin{array}{l}\text { UAS-puml-PA- } \\
\text { S190N }\end{array}$ & R. Kühnlein \\
\hline RKF1240 & $\begin{array}{l}w^{*} ; P\{U A S T a t t B-p u m l-P A\} a t 86 F b \# 2 / T M 3, S b[1] \\
\text { float }\end{array}$ & 1,3 & UAS-puml-PA & R. Kühnlein \\
\hline RKF1242 & $\begin{array}{l}w^{*} ; P\{U A S T a t t B-p u m l-P A-S 190 N-D 371 A\} a t 86 F b \\
\# 1 / T M 3, S b[1] \text { float }\end{array}$ & 1,3 & $\begin{array}{l}\text { UAS-puml-PA- } \\
\text { S190N-D371A }\end{array}$ & R. Kühnlein \\
\hline RKF1244 & $\begin{array}{l}w^{*} ; P\{U A S T a t t B-p u m l-P A-D 371 A\} a t 86 F b \# 1 / \\
\text { TM3, Sb[1] float }\end{array}$ & 1,3 & $\begin{array}{l}\text { UAS-puml-PA- } \\
\text { D371A }\end{array}$ & R. Kühnlein \\
\hline PHF1290 & $\begin{array}{l}w\left[^{*}\right] ; P\{w[+m W . h s]=G a w B\} F B+S N S, \text { puml1/CyO; } \\
+/+\end{array}$ & 1,2 & FB-GAL4 in puml ${ }^{1}$ & this work \\
\hline PHF1291 & $\begin{array}{l}\left.w{ }^{*}\right] ; P\{w[+m C]=A c t 5 C-G A L 4\} 25 F O 1, p^{2} l^{1} / C y O ; \\
+/+\end{array}$ & 1,2 & $\begin{array}{c}\text { Act5C-GAL4 in } \\
\text { puml }\end{array}$ & this work \\
\hline PHF1292 & $w[*] ; P[G A L 4] c 724, C G 1882[1] / C y O ;+/+$ & 1,2 & $\begin{array}{c}\text { c724-GAL4 in } \\
\text { puml }\end{array}$ & this work \\
\hline RKF1284 & $w^{*} ;$ puml ${ }^{1} ; P\{U A S T a t t B-p u m l-P A\} a t$ 86Fb \#1 & $\begin{array}{c}1,2 \\
3\end{array}$ & $\begin{array}{l}\text { UAS-puml-PA in } \\
\text { puml } \\
\end{array}$ & $\begin{array}{c}\text { (Rosenberg, } \\
\text { 2012) }\end{array}$ \\
\hline RKF532 & $\begin{array}{l}w^{*} ; P\{w[+m C] b m m[S c e r \mid U A S]=U A S-b m m\} \# 2 c ;+ \\
/+\end{array}$ & 1,2 & UAS-bmm & $\begin{array}{c}\text { (Grönke et al., } \\
\text { 2005b) }\end{array}$ \\
\hline RKF534 & $w^{*} ; P\{w[+m C] U A S T-b m m:: E G F P\} / C y O$ float & 1,2 & UAS-bmm::eGFP & $\begin{array}{c}\text { (Grönke et al., } \\
2005 b)\end{array}$ \\
\hline JBF1454 & $w^{1118} ;+/+; P\{G D 5139\} v 37880$ & 1,3 & $U A S-b m m-R N A i$ & VDRC37880 \\
\hline RKF1684 & $w^{1118} ; D f(2 R) B S C 265 / C y O$ & 1,2 & $\begin{array}{l}\text { among others } \\
\text { puml-Deficiency }\end{array}$ & BDSC 23164 \\
\hline RKF1402 & $\begin{array}{l}y[1] s c\left[^{*}\right] v[1] ; P\{y[+t 7.7] \\
v[+t 1.8]=T R i P . H M S 02650\} a t t P 40 / C y O(\text { floating) }\end{array}$ & 1,2 & UAS-puml-RNAi & BDSC 42957 \\
\hline JRF1252 & $+/+; P[G A L 4] c 724 ;+/+$ & 2 & $\begin{array}{l}\text { c724-GAL4; } \\
\text { Stellate cell }\end{array}$ & $\begin{array}{c}\text { (Sözen et al. } \\
\text { 1997) }\end{array}$ \\
\hline JRF1253 & $+/+; P[$ Gal4] $c 42 ;+/+$ & 2 & $\begin{array}{c}\text { c42-GAL4, } \\
\text { Principle cell }\end{array}$ & $\begin{array}{c}\text { (Sözen et al. } \\
\text { 1997) }\end{array}$ \\
\hline JRF1254 & $+/+;$ UO-Gal4/CyO float; +/+ & 2 & $\begin{array}{c}\text { UO-GAL4 } \\
\text { Malpighian tubule }\end{array}$ & $\begin{array}{c}\text { (Terhzaz et al. } \\
\text { 2010) }\end{array}$ \\
\hline PHF1740 & $w^{*} ; p u m I^{1} / C y O_{\text {floating }} ;$ bmm1 $1^{1} / T M 3$, Ser $^{*}$ & $\begin{array}{c}1,2 \\
3\end{array}$ & $\begin{array}{c}\text { puml }{ }^{1}, b_{m} m^{1} \\
\text { double mutant }\end{array}$ & this work \\
\hline PHF1741 & $w^{*} ;+1+; U A S-p u m I-P A, b m m^{1}$ & 1,3 & $\begin{array}{c}\text { UAS-puml in } \\
\text { bmm } m^{1}\end{array}$ & this work \\
\hline JRF1250 & $\begin{array}{l}w^{1118} ; \text { puml }{ }^{1} P\left\{w^{+m c} \text { puml.puml:mCherry }\right\} \# 13 A ; \\
+/+\end{array}$ & 1,2 & $\begin{array}{l}\text { puml }{ }^{1} \text { genomic } \\
\text { rescue }\end{array}$ & $\begin{array}{c}\text { (Rosenberg, } \\
\text { 2012) }\end{array}$ \\
\hline
\end{tabular}




\begin{tabular}{|c|c|c|c|c|}
\hline JRF1233 & $\begin{array}{l}W^{*} ; P\left\{w^{+m c} \text { puml.puml:::mCherry\}\#13A; }\right. \\
P\left\{w^{+m c} \text { puml.puml:::mCherry\}\#16A }\right.\end{array}$ & $\begin{array}{c}1,2 \\
3\end{array}$ & $\begin{array}{l}\text { pum }{ }^{1}{ } \text {,double" } \\
\text { genomic rescue }\end{array}$ & $\begin{array}{c}\text { (Rosenberg, } \\
\text { 2012) }\end{array}$ \\
\hline RKF1288 & $w^{*} ;$ puml $I^{1}$, UO-Gal4/CyO floating & 1,2 & UO-GAL4 in puml ${ }^{1}$ & $\begin{array}{l}\text { (Rosenberg, } \\
\text { 2012) }\end{array}$ \\
\hline RKF972 & $w^{1118}$ bclV & 1 & white mutant & Grönke 2009 \\
\hline SGF717 & $w^{*} ;\left.D m H s\right|^{1}$ & 1,2 & Hsl mutant & S. Grönke \\
\hline PHF1484 & $w^{*} ; D m H s I^{1}$ & 1,2 & $\begin{array}{c}\text { Hsl mutant, } \\
\text { backcrossed into } \\
\text { RKF972 }\end{array}$ & this work \\
\hline SGF830 & $\begin{array}{l}W^{*} ; P\left\{W^{+m C} d H s / S c e r|U A S=U A S-d H s|\right\} \# 17 b \text { orange / } \\
T M 3 S b_{\text {float }}^{*}\end{array}$ & 1,3 & UAS-DmHsl & $\begin{array}{l}\text { (Grönke, } \\
\text { 2005) }\end{array}$ \\
\hline SGF831 & $\begin{array}{l}w^{*} ; P\left\{w^{+m c} d H s \mid I^{\text {scerlUAS }}=U A S-d H s l-e G F P\right\} \# 11 a / \\
C y O_{\text {float }}\end{array}$ & 1,2 & UAS-DmHsl::eGFP & $\begin{array}{c}\text { (Grönke, } \\
\text { 2005) }\end{array}$ \\
\hline RKF1421 & $w^{*} ;+/+; P\{L p p-G A L 4 . B\} c 4 / T M 3, S b^{*}$ float. & 1,3 & $\begin{array}{l}\text { Lpp }>\text { GAL4 fat } \\
\text { body specific }\end{array}$ & $\begin{array}{c}\text { (Brankatschk } \\
\text { und Eaton } \\
\text { 2010) }\end{array}$ \\
\hline RKF910 & $\begin{array}{l}W^{*} ; P\left\{W^{+m W . h s}=\text { GawB }\right\} F B+S N S / C y O \text { float. ; plin } 1^{1} / \\
T M 6 C, S b^{1} T b^{1} \text { float. }\end{array}$ & $\begin{array}{c}1,2 \\
3\end{array}$ & $\begin{array}{c}F B-S N S>G A L 4 \text { fat } \\
\text { body driver in } \\
\text { plin1 mutant }\end{array}$ & $\begin{array}{l}\text { (Beller et al., } \\
\text { 2010) }\end{array}$ \\
\hline RKF649 & $w^{*} ;+/+; p \operatorname{plin} 1^{1} / T M 3 S b^{*} e^{*}$ float & 1,3 & plin1 mutant & $\begin{array}{l}\text { (Beller et al., } \\
\text { 2010) }\end{array}$ \\
\hline BDSC33001 & $\begin{array}{l}\mathrm{y}[1] s c\left[^{*}\right] v[1] ; P\{y[+t 7.7] v[+t 1.8] \\
=\text { TRiP.HMSO0801\}attP2 }\end{array}$ & 1,2 & Cyp1 RNAi & BDSC 33001 \\
\hline RKF1583 & $w^{*} ; P\{U A S T-C y p 1:: e G F P\} a t t P 40 \# 2 M$ & 1,2 & UAS-Cyp1::eGFP & $\begin{array}{l}\text { R. Kühnlein } \\
\text { and A. Sahu }\end{array}$ \\
\hline RKF1693 & $w[1118] ; P\{U A S T-C y p 1:: e G F P\} a t t P 40 ; p \operatorname{lin} 1[1]$ & $\begin{array}{c}1,2 \\
3\end{array}$ & $\begin{array}{c}\text { Cyp1::eGFPplin1 } \\
\text { mutant }\end{array}$ & R. Kühnlein \\
\hline RKF1720 & $w^{1118} ;+/+;$ Cyp ${ }^{\text {EP1073 }}$, plin1 ${ }^{1}$ & 1,3 & $\begin{array}{l}\text { Cyp1 plin1 double } \\
\text { mutant }\end{array}$ & R. Kühnlein \\
\hline $\begin{array}{l}\text { BDSC10136 } \\
\text { (RKF1692) }\end{array}$ & $W^{1118} P\left\{w^{+m C}=E P\right\} C y p 1^{E P 1073}$ & 1 & $\begin{array}{l}\text { Cyp1 mutant } \\
\left(\text { Cyp1 } 1^{1}\right)\end{array}$ & $\begin{array}{l}\text { (Spradling et } \\
\text { al. 1999) }\end{array}$ \\
\hline PHF1746 & $\begin{array}{l}W^{1118} P\left\{W^{+m C}=E P\right\} C y p 1^{E P 1073} ; P\{U A S T- \\
\text { Cyp1::eGFP\}attP40; }\end{array}$ & 1,2 & $\begin{array}{l}\text { UAS-Cyp1 in Cyp1 } \\
\text { mutant }\end{array}$ & R. Kühnlein \\
\hline PHF1747 & $\begin{array}{l}W^{1118} P\left\{w^{+m C}=E P\right\} C y p 1^{E P 1073 ;+/+;} P\{L p p- \\
G A L 4 . B\} C 4 / T M 3, S b^{*} \text { float. }\end{array}$ & 1,3 & $\begin{array}{l}\text { Lpp }>\text { GAL4 in Cyp1 } \\
\text { mutant }\end{array}$ & R. Kühnlein \\
\hline PHF1743 & $\begin{array}{l}\text { w*; P }\{w+m C=A c t 5 C-G A L 4\} 25 F O 1 / C y O ; \text { UAS- } \\
\text { Ptsl::EYFP / TM3,Ser* }\end{array}$ & $\begin{array}{c}1,2 \\
3\end{array}$ & $\begin{array}{c}\text { Act5c>GAL4, UAS- } \\
\text { Ptsl::EYFP }\end{array}$ & this work \\
\hline PHF1749 & $\begin{array}{l}w^{*} ; P\{w[+m C] d H s I[S c e r \mid U A S]=U A S-d H s l- \\
E G F P\} \# 11 a ; p l i n 1[1]\end{array}$ & $\begin{array}{c}1,2 \\
3\end{array}$ & $\begin{array}{l}\text { UAS-DmHsl::EGFP } \\
\text { in plin1 mutants }\end{array}$ & this work \\
\hline PHF1750 & $\begin{array}{l}\left.y[1] s c{ }^{*}\right] v[1] ; P\{y[+t 7.7] \\
[+t 1.8]=T R i P . H M C 03624\} a t t P 40\end{array}$ & 1,2 & $\begin{array}{c}\text { UAS-CG17597- } \\
\text { RNAi }\end{array}$ & BDSC52886 \\
\hline PHF1751 & $\begin{array}{l}y[1] s c\left[{ }^{*}\right] v[1] ; P\{y[+t 7.7] \\
v[+t 1.8]=T R i P . H M C 03956\} a t t P 40\end{array}$ & 1,2 & $\begin{array}{l}\text { UAS-CG12512- } \\
\qquad \text { RNAi }\end{array}$ & $\begin{array}{c}\text { BDSC } \\
55269201\end{array}$ \\
\hline PHF1752 & $\begin{array}{l}y[1] v[1] ; P\{y[+t 7.7] \\
v[+t 1.8]=T R i P . H M C 03224\} a t t P 40 / C y O\end{array}$ & 1,2 & $\begin{array}{l}\text { UAS-CG17320- } \\
\text { RNAi }\end{array}$ & BDSC 51479 \\
\hline PHF1753 & $\begin{array}{l}y[1] s c\left[^{*}\right] v[1] ; P\{y[+t 7.7] \\
v[+t 1.8]=T R i P . H M C 04810\} a t t P 2\end{array}$ & 1,2 & UAS-Pex16-RNAi & BDSC 57495 \\
\hline
\end{tabular}

\subsection{Physiology}

\subsubsection{Lifespan}

Lifespan assays were performed, in order to analyse possible biological effects of the interfered genes used in this study. For this, male flies were collected in the first $24 \mathrm{~h}$ after eclosure (derived from a density seeding with 150 embryos/midsize vial) and kept under standard adult feeding conditions for ten days (40 flies / midsize vial; 
12h/12h-light/dark cycle). Flies were transferred into a new vial every second day. Then, all intact and viable flies were transferred into new midsize vials ( 40 flies / vial). Flies were kept upright and food was exchanged every 2,5 days (change on early Monday, Wednesday and late Friday; kept at $25^{\circ} \mathrm{C} ; 12 \mathrm{~h} / 12 \mathrm{~h}$-light/dark cycle; 60\% relative humidity). Dead animals mostly stuck to the old food and were not transferred to a new vial during food replacement. Dead flies were scored at the day of food change until all flies died. Each tested genotype was kept in six replicates ( $n_{\text {total population }}=240$ male flies). In order to avoid positional effects of the vial or bias each vial obtained an arbitrary number and positions were alternated randomly within the box after each food exchange. Survival analysis was performed in OriginPro 9.1 using the Kaplan-Meier analysis and a Log Rank test.

\subsubsection{Capillary feeding (CAFÉ) assay for quantification of food uptake}

Prandial behaviour of male flies was addressed by measuring food intake for six days, using six-day-old adult male flies, in a modified CAFÉ system (Ja et al., 2007) at ad libitum feeding condition. Male flies, deriving from controlled density seeding (150 embryos/ midsize vial) and collection within the first 24 hours after eclosure. 40 flies were kept in a midsize vial for the following days. For the assay, flies were then transferred into individual chambers of the CAFÉ system (based on a 24-well cell culture plate) and kept at very high humidity $(\sim 100 \%)$ at $25^{\circ} \mathrm{C}$ at a $12 \mathrm{~h} / 12 \mathrm{~h}$-light/dark cycle. To exclude positional effects, flies from the different tested genotypes were allocated randomly to the chambers. One chamber of each plate was dedicated for the evaporation control at random and no fly was put into this chamber. The liquid diet ( $5 \%$ sucrose, $5 \%$ yeast extract in $\mathrm{H}_{2} \mathrm{O}$ ) was provided in $5 \mu \mathrm{L}$ capillaries (ring caps, Hirschmann, Cat. \#: 9600105). Capillaries were substituted every day and measured volume of consumed food was corrected by the evaporation control. Statistical analysis of the food intake was performed in Origin Pro 9.1 as indicated in the figure caption.

\subsubsection{Lipogenesis experiment}

Lipogenesis in adult flies was followed by incorporation of Glucose D-[14C(U)] into neutral lipids(Katewa et al. 2012; 2012). Adult flies (10 days after eclosure; from density seeding; 150 embryos/midsize vial, cohorts of 40 males after eclosure; food 
changed every second day; $25^{\circ} \mathrm{C} ; 12 / 12 \mathrm{~h}$ light/dark-cycle, $50 \%$ humidity) were transferred to $1 \%$ Agar with an $200 \mu \mathrm{L}$ gel-block of labelled food: $5 \%$ Yeast extract, $5 \%$ Sucrose, $1 \%$ Agar and additional $325 \mathrm{mM}$ Glucose with $2 \mu \mathrm{Ci}{ }^{14} \mathrm{C}$-labeled Glucose (Glucose D-[14C(U)], Perkin Elmer, NEC042X050UC [Glucose dissolved in 500 $\mu \mathrm{L}$ Ethanol by manufacturer]).

A first set of flies was collected after $24 \mathrm{~h}$ (pulse) and another after $60 \mathrm{~h}$ (chase; kept on unlabelled food after the $24 \mathrm{~h}$ "pulse"-period) - snap frozen in liquid nitrogen. The frozen samples ( 20 flies per replicate, 3 replicates per genotype and time point) were homogenized in $1,5 \mathrm{~mL}$ chloroform. Lipids were fractionated by solid phase extraction (SPE) using DSC-NH2 columns (DISCOVERY DSC-NH2 6mL Tube 1GM, Sigma Aldrich, Cat. \#: 52640-U) (Kaluzny et al., 1985).

Homogenates were loaded on the columns and flow through was discarded. Neutral lipids were eluted with 1,5mL Chloroform:2-Propanol (2:1). Then fatty acids were eluted with $2 \%$ Acetic acid in diethyl ether (1,5mL used). In a last step, Phospholipids (PLs) were eluted with 1,5mL Methanol (100\%). Elution buffers should be prepared freshly to ensure high extraction yield and very high specificity to targeted lipid class. Solvents from the lipid fractions were evaporated under a stream of nitrogen $(\mathrm{g})$.

After that, lipids were resuspended in scintillation fluid (Ultima Gold scintillation cocktail, Perkin Elmer, Cat. \#:6013326) and radiometrically analysed with a Liquid Scintillation Analyzer (Tri-Carb 2100TR, Packard). Statistical analysis was performed with OriginPro9.1.0 as indicated in the figure caption.

\subsubsection{Osmotic stress resistance}

In order to address the survivability under osmotic stress, adult male flies from controlled density (150 embryos / midsize vial) were collected at the day of eclosure and kept for six days under standard feeding conditions (40 flies / midsize vial; $12 \mathrm{~h} / 12 \mathrm{~h}$-light/dark cycle; $60 \%$ relative humidity). Flies were then transferred ( 25 flies / vial) to standard food that was supplemented with additional $4 \%$ sodium chloride $(\mathrm{NaCl})$ and kept as described before. Food was changed every second day. Dead flies were scored at least every 24 hours. Kaplan-Meier analysis and Log Rank test for statistical comparison of the survival times were performed in OriginPro 9.1. 


\subsubsection{Starvation resistance}

Total starvation was performed with adult male flies (six days after eclosure) deriving from density seedings (150 embryos / midsize vial). Flies were kept under standard feeding conditions (40 flies / midsize vial; 12h/12h-light/dark cycle). To address starvation resistance, flies were transferred into new vials (25 flies / vial) or into individual tubes of the Drosophila activity monitor (DAM) system. Water was supplied in form of $2 \%$ agarose gel. Flies were kept at $25^{\circ} \mathrm{C}, 12 \mathrm{~h} / 12 \mathrm{~h}$-light/dark cycle and $\sim 60 \%$ relative humidity. Dead flies were scored by manual counting (in vials) or by the last time point of measured activity in the DAM-system. Survival analysis was performed in OriginPro 9.1 using the Kaplan-Meier analysis and a Log Rank test.

\subsubsection{Desiccation}

Desiccation resistance was addressed in adult male flies (six days after eclosure, from density seeding with 150 embryos / midsize vial, 12/12h-light/dark cycle; 60\% relative humidity on standard food). Flies were transferred into an empty midsize (20 flies / vial) vial or as individuals into a tube of the Drosophila activity monitor (DAM) system and were kept at $25^{\circ} \mathrm{C}$, a $12 / 12 \mathrm{~h}$-light/dark cycle and $60 \%$ relative humidity. Dead flies were scored by manual counting (in the vials) or by the last time point of detected activity in the DAM-system. Survival analysis was performed in OriginPro 9.1 using the Kaplan-Meier analysis and a Log Rank test.

\subsubsection{Metabolic Rate}

Metabolic rate was determined as described in (Yatsenko et al., 2014). In detail, 3 male flies (six-day-old from density seeding with 150 embryos / midsize vial kept at $12 \mathrm{~h} / 12 \mathrm{~h}$-light/dark cycle and $60 \%$ relative humidity on standard food) were placed in one freshly build measurement chamber. The chamber was assembled by adding a $50 \mu \mathrm{L}$ capillary to the tip of a $1 \mathrm{~mL}$ plastic pipette tip. The junction was sealed airtight with glue (Power-Pritt-Gel, Henkel). Then a small piece of foam/cotton wool was placed inside the chamber and Soda lime was added on top to adsorb the generated $\mathrm{CO}_{2}$ generated by the flies. Another piece of foam was added to separate the flies from soda lime. After loading of the chamber with three flies, it was sealed airtight with modelling clay (Künstlerbedarf Schulze). The metabolic rate chamber was then placed in a thin layer chromatography (TLC) chamber. The capillary tip was 
submerged into Eosin $/ \mathrm{H}_{2} \mathrm{O}$-solution and images were taken every $30 \mathrm{~min}$ for a period of three hours. In order to avoid artefacts, let the chambers settle for $5 \mathrm{~min}$ after the start of the experiment and start image acquisition. It is crucial that TLC- and metabolic rate-chambers are equilibrated to the same temperature. Ensure constant atmospheric conditions during the measurement (experiments were performed in a fly incubator). Consumed oxygen was measured indirectly by the generated $\mathrm{CO}_{2}$ that was absorbed by the soda lime thereby reducing the gas volume in the measurement chamber. The pressure difference was balanced draft of the coloured water. Images were analysed in Image J. For each picture the white markings on the capillaries were used to set a scale for the picture. The volume was calculated by the difference in volume from the black marking (representing the calibrated $50 \mu \mathrm{L}$ scale for the capillary) to the meniscus from two following time-points. For each tested condition/genotype 3-6 replicates were measured at three different time points (zeitgeber $+0 \mathrm{~h},+4 \mathrm{~h}, 8 \mathrm{~h}$ ). For normalization one metabolic rate chamber was incubated with three dead flies. Calculations were performed in Excel and results were analysed statistically in OriginPro 9.1 as indicated in the figure caption.

\subsubsection{Startle induced climbing assay}

In order to address the startle induced climbing activity flies were scored as described in (Greene et al., 2003).

In detail, 20 male flies (from density seeding 150 embryo/ midsize vial; 12/12h light/dark cycle; $60 \%$ humidity; standard food; cohorts of 40 flies per midsize vial after eclosure) were placed in the first chamber of a counter current apparatus (Benzer, 1967). Flies were tapped down, then given a time frame of 30 s to climb a distance of $10 \mathrm{~cm}$. Successors were transferred into the second vial and the cycle was repeated. After five replications flies were counted in the six vials. The climbing index was calculated as:

$$
\text { Climbing index }=\frac{\sum(\# \text { flies } \times \text { index } \# \text { vial })}{4 \times \text { total flies in assay }}
$$


Measurements were repeated as duplicates per genotypes and repeated in four independent experiments. Calculations were performed in Excel. Statistical analysis was executed in OriginPro 9.1 as indicated in the figure caption.

\subsubsection{Fecundity assay}

Fecundity was measured by the scoring of egg laying of individual females during over a period of 25 days. Virgins were collected from a density seeding and individual virgins were paired with 2 male flies and kept in a small fly vial for $48 \mathrm{~h}$. The males were then removed and females were kept alone in small vials for the next six days. Then again 2 male flies were paired with the female for another 2 days. This cycle was repeated four times. Flies were transferred to a new vial (with standard fly food and a drop of yeast $(\sim 200 \mu L))$ every day. Laid eggs were counted under a stereo-microscope. Fecundity is expressed as mean cumulative number of laid eggs per female. Statistical analysis was performed as described in the figure caption.

\subsubsection{Hatchability assay}

Eggs laid by females in the fecundity assay were kept for $48 \mathrm{~h}$ at $25^{\circ} \mathrm{C}$. Then hatched eggs were scored and compared to the total number of laid eggs to get $\%$ of hatched eggs. Statistical analysis was performed as described in the figure caption.

\subsubsection{Viability assay}

Vials from the hatchability assay were kept for ten additional days at $25^{\circ} \mathrm{C}$ and number of pupae and eclosed flies (empty pupae counted) were scored. Statistical analysis was performed as described in the figure caption.

\subsubsection{Coupled colorimetric assay (CCA) for lipid determination}

Lipids were measured as described in (Hildebrandt et al., 2011).

\subsubsection{Generation of fly homogenates}

4 replicas of 5 flies were collected in 1,2mL collection tubes (Qiagen, Cat. \#: 19560; Caps: Cat. \#: 19566) and immediately snap frozen in liquid nitrogen and either stored at $-20^{\circ} \mathrm{C}$ or processed directly. To each replicate a $5 \mathrm{~mm}$ metal bead and $600 \mu \mathrm{L}$ homogenization buffer $\left(0,05 \%\right.$ Tween in $\left.\mathrm{H}_{2} \mathrm{O}\right)$ was added. Samples were then homogenized with a mixer mill (Retsch, MM400) at $30 \mathrm{~s}^{-1}$ for $45 \mathrm{sec}$. and heatinactivated in a water bath $\left(70^{\circ} \mathrm{C}, 5 \mathrm{~min}\right)$. After that, samples were pelleted $(2500 \mathrm{xg}$; 
$5 \mathrm{~min}$ ) and the supernatant was transferred to a $1 \mathrm{~mL}$ Master block (96-well, Greiner bio-one) and covered with Silver seal sealer (Aluminium, Greiner bio-one). Homogenates were stored at $-20^{\circ} \mathrm{C}$ or used directly.

\subsubsection{Lipid determination}

Homogenates were preheated to $37^{\circ} \mathrm{C}$, mixed and pelleted $(2500 \times \mathrm{g}, 5 \mathrm{~min})$. A $50 \mu \mathrm{L}$ aliquot of homogenate was used for lipid and protein measurement. Supernatant was transferred into a 96-well microtest plate (Sarstedt, Cat. \#: 82.1581) and absorbance was measured at $540 \mathrm{~nm}$ to get the baseline absorbance. Afterwards 200 $\mu \mathrm{L}$ of CCA-Mix (Triglycerides; Microgenics, Cat. \#: 981786) for lipids. Samples were incubated at $37^{\circ} \mathrm{C}$ for $30 \mathrm{~min}(120 \mathrm{rpm})$. Finally, samples were re-measured at 540nm. Absorbance values were then corrected by the baseline absorbance. In order to quantify the amount of lipid Thermo Trace Triglycerides standards (used 0, 5.5, 11, $22,33,44 \mu \mathrm{g}$ in $50 \mu \mathrm{L}))$ solved in homogenization buffer were used to generate a standard curve. Lipid data were normalized to protein levels measured separately from the same fly homogenate. Data were analysed as indicated in the figure captions.

\subsubsection{Protein determination}

Homogenates were preheated to $37^{\circ} \mathrm{C}$, mixed and pelleted $(2500 \times \mathrm{g}, 5 \mathrm{~min})$. A $50 \mu \mathrm{L}$ aliquot of homogenate was used for protein measurement. Supernatant was transferred into a 96-well microtest plate (Sarstedt, Cat. \#: 82.1581) and absorbance was measured at $570 \mathrm{~nm}$. Afterwards $200 \mu \mathrm{L}$ of BCA-Mix (prepared according too manual; BCA Protein Assay Kit; Pierce, Thermo Scientific; Cat. \#: 23225) were added. Samples were incubated at $37^{\circ} \mathrm{C}$ for $30 \mathrm{~min}(120 \mathrm{rpm})$ and absorbance was measured again (at $570 \mathrm{~nm}$ ). In order to quantify the amount of protein BSA standards (provided

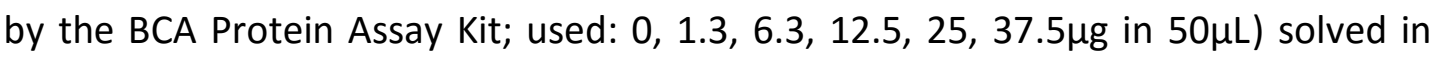
homogenization buffer were used to generate a standard curve.

\subsubsection{Non-esterified fatty acid (NEFA) assay}

Non-esterified fatty acids (NEFAs) were measured colorimetrically with the HR Series

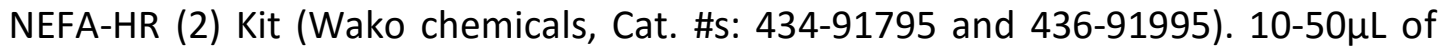
sample were transferred into a 96-well microtest plate and absorbance was 
measured at $552 \mathrm{~nm}$ to get the baseline before the reaction. Then, $150 \mu \mathrm{L}$ of NEFA R1 Mix were added and samples were incubated at $37^{\circ} \mathrm{C}(10 \mathrm{~min}, 120 \mathrm{rpm})$. Afterwards, $75 \mu \mathrm{L}$ of NEFA R2 solution were added and samples were incubated again at $37^{\circ} \mathrm{C}$ (10min, 120rpm; avoid strong light exposure). Finally, absorbance at 552nm was measured a second time (T30). In order to quantify the amount of NEFAs, oleic acid was solubilized in $\mathrm{H}_{2} \mathrm{O}$ with $0,05 \%$ Tween and used as standard $(0,0.25,0.625,1.25$, $2.5,3.75,5,7.5$ and $14 \mu \mathrm{g}$ ). With an additional protein measurement (see BCA-assay in CCA-Assay paragraph) NEFA data could be normalized. Independent experiments were performed at least three times. Data were analysed as indicated in the figure captions.

\subsubsection{Carbohydrate analysis}

Fly homogenates for Glycogen measurements were prepared as described before (2.5.12.1). Avoid multiple freezing of homogenates. Glycogen measurements were performed by using Amyloglucosidase (Amyloglucosidase from A. niger, Sigma, Cat. \#: A1602-25MG) for glycogen hydrolysis followed by the detection of free glucose (Glucose Assay (GO) Kit, Sigma, Cat.: GAGO20-1KT) as described in (Tennessen et al., 2014). Homogenates from fed animals were diluted 1:3 in homogenization buffer whereas homogenates derived from starved animals were used directly for the assay. This is necessary to ensure that the detected glucose amounts are within the linear range of the assay $(0,25-\sim 5 \mu \mathrm{g})$.

Homogenates were measured in two separate reactions - one with added amyloglucosidase (total glucose determination) and a second without the enzyme (free glucose). $30 \mu \mathrm{L}$ of the un-/diluted homogenate were transferred into a Microtest plate 96-well (Sarstedt, Cat. \#: 82.1581) and absorbance was measured (T0) at $540 \mathrm{~nm}$. Afterwards $100 \mu \mathrm{L}$ of GO Assay mix was added to each sample (in the first reaction with additional $0.3 \mathrm{U}$ amyloglucosidase $(1 \mu \mathrm{L}$ from $4,3 \mathrm{mg} / \mathrm{mL}$ stock solution, lyophilized powder $\sim 70 \mathrm{U} / \mathrm{mg}$, Sigma, 10115-1G-F). Samples were incubated at $37^{\circ} \mathrm{C}$ for $30 \mathrm{~min}(120 \mathrm{rpm})$. Then $100 \mu \mathrm{L}$ of $12 \mathrm{~N} \mathrm{H}_{2} \mathrm{SO}_{4}$ were added to terminate the reaction and enable colour development (stable end product quantified) and absorbance was measured again at 540nm (T30). Final absorbance of the samples was corrected by the measured TO values. 
In order to quantify the amount of glycogen glucose (D-(+)-Glucose, Sigma, G8270100G) and bovine liver glycogen (Glycogen from bovine liver - Type IX, Sigma, G08851G; used: $0,0.3,0.6,1.2,2.4,4.8 \mu \mathrm{g}$ ) were used to generate a standard curve. Total glycogen was calculated by the subtraction of the free glucose amount and was normalized to protein content. Measurements were performed as quadruplicates of 5 flies and repeated at least three times. Data were analysed as indicated in the figure captions.

\subsubsection{Body weight measurements}

Wet weight of flies was measured in adult male flies (6-day-old; deriving from density seeding; 150 embryos/midsize vial; cohorts of 40 males after eclosure; food changed every second day; $25^{\circ} \mathrm{C} ; 60 \%$ humidity; $12 / 12 \mathrm{~h}$ light/dark cycle). Flies were collected and snap frozen in liquid nitrogen. For weighing flies were equilibrated to room temperature. Cohorts of ten flies (at least 3 technical replicates) were measured on a weighing scale (Mikrowaage MC5, Sartorius). Each cohort was measured three times and average weight was calculated afterwards. Weighing was performed in three independent experiments. Statistical analysis was done in OriginPro 9.1.0 as indicated in the figure caption.

\subsubsection{TLC analysis of neutral lipids}

Thin layer chromatographic (TLC) of the lipid content of collected samples was performed with small modifications as described by Baumbach et al. (Baumbach et al., 2014b). In general, lipids were extracted according to Bligh and Dyer (Bligh and Dyer, 1959). Three biological replicates of five flies each were homogenized in $285 \mu \mathrm{L}$ buffer $\left(150 \mu \mathrm{L}\right.$ methanol, $75 \mu \mathrm{L}$ chloroform, $\left.60 \mu \mathrm{L} \mathrm{H}_{2} \mathrm{O}_{\mathrm{dd}}\right)$ were homogenized by ten $1,4 \mathrm{~mm}$ ceramic beads (Peqlab) using a mixer mill (Retsch, MM400) at $30 \mathrm{~s}^{-1}$ for $45 \mathrm{sec}$. Afterwards, samples were incubated in a water bath $\left(1 \mathrm{~h}, 37^{\circ} \mathrm{C}\right)$. In the next step, first $75 \mu \mathrm{L}$ chloroform and then $75 \mu \mathrm{L} \mathrm{KCl}(1 \mathrm{M})$ were added to the samples (vortexed for 20s). Subsequently, phase separation was achieved by centrifugation (1000 x g, $2 \mathrm{~min}$ ). The organic phase (lower) was collected in a new $1,5 \mathrm{~mL}$ tube. Solvent was evaporated in a SpeedVac concentrator $(7 \mathrm{~min})$. Lipid pellets were stored at $-20^{\circ} \mathrm{C}$ or used directly. 
Lipid pellets from control flies were resuspended in $100 \mu \mathrm{L}$ buffer (chloroform: methanol, 1:1). Buffer volumes of samples were normalized to protein amounts of control flies. Protein amounts were determined from sibling flies as described in chapter (2.5.12.3)

Finally, $20 \mu \mathrm{L}$ of each sample were applied on a high performance thin layer chromatography (HPTLC) plate (Merck, Cat. \#: 105633). For lipid class assignment following lipid standards were used: $40 \mu \mathrm{g}$ of glyceryltrioleate, $40 \mu \mathrm{g}$ of 1,3-diolein, $40 \mu \mathrm{g}$ of 1,2-dioleoyl rac-glycerol, $40 \mu \mathrm{g}$ of mono-olein (provided as mix in SUPELCO Mono-, Di-, Triglyceride Mix, SIGMA 1787-1AMP) supplemented with $4 \mu \mathrm{g}$ oleic acid (FA; CALBIOCHEM \#4954).

Lipids were separated using n-hexane / diethyl ether / acetic acid (70:30:1, v/v/v; Merck) as mobile phase. Running phase was stopped $\sim 1 \mathrm{~cm}$ before the mobile phase reached the border of the TLC-plate followed by air-drying of the plated. Afterwards, plates were immersed in $8 \%(\mathrm{w} / \mathrm{w}) \mathrm{H}_{3} \mathrm{PO}_{4}$ containing $10 \%(\mathrm{w} / \mathrm{v})$ copper (II) sulphate pentahydrate. Excess liquid on the glass-carrier was removed and plates were then charred for $\leq 5 \mathrm{~min}\left(180^{\circ} \mathrm{C}\right)$, avoiding too much background. After cooling, plates were finally imaged using a Canon LiDE220 scanner. Amounts of lipids were calculated by comparison to loaded standards on the same plate both measure by densitometry using ImageJ v1.49m.

\subsubsection{Lipidomics analysis of Malpighian tubules}

Lipidomic analyses were performed to identify which lipid species were accumulated in Malpighian tubules of puml ${ }^{1}$ in comparison to control flies. The flies used for these experiments derived from a density seeding (150 larvae/ midsize vial) and were kept at standard conditions. 6d old flies were dissected in cold Ringer's solution using forceps. Malpighian tubules were removed from the intestinal tissue by pulling away the ureter. Tissue was collected in Ringer's solution and pelleted $\left(4^{\circ} \mathrm{C}, 1000 \times \mathrm{g}\right.$, $10 \mathrm{~min})$. Afterwards, the buffer was removed by careful pipetting. Samples were snapfrozen in liquid nitrogen and stored at $-20^{\circ} \mathrm{C}$.

For the pilot experiment $5 \times 10$ Malpighian tubule pairs per genotype were analysed. Lipid extraction and mass spectrometry and lipid annotation was performed as 
described by Hoffereck (Hofferek, 2016). Additional analyses were performed using $3 \times 100$ Malpighian tubule pairs per genotype. Lipid extraction, mass spectrometry and lipid annotation was performed as described in Knittelfelder et al. (Knittelfelder et al., 2014). Calculations for relative comparison of TAG species were performed in MS Excel 2013. Graphs and heat maps were generated using OriginPro9.1.0.

\subsection{Microscopy}

\subsubsection{Used fluorophores, dyes and concentrations}

If not stated otherwise, for standard imaging sampled were explanted in 1X PBS and directly mounted on microscope slides (Menzel-Gläser, Thermo Scientific, AAAA000001\#\#12E) in the staining solution. Samples were not fixed. Images were acquired within 1 hour after mounting. All samples were covered with a small cover slide (10mm, circular, \#1, Menzel-Deckgläser, VWR; CAT. \#:631-1340) that was sealed by nail polish. For embedding 1 X PBS was used containing the following dyes:

Table 5 Dyes and fluorophores used for laser scanning microscopy

\begin{tabular}{|c|c|l|l|}
\hline Dye/Fluorophore & $\begin{array}{c}\text { Excitation/Emission } \\
(\mathbf{n m})\end{array}$ & \multicolumn{1}{c|}{ Concentration } & \multicolumn{1}{|c|}{ Manufacturer } \\
\hline DAPI & $405 / 415-470$ & $1: 1000(6,3 \mathrm{mM})$ & Invitrogen; D1306 \\
\hline Cellmask & $633 / 635-670$ & $1: 1000(5 \mathrm{mg} / \mathrm{mL})$ & Invitrogen; C10046 \\
\hline Bodipy493/503 & $488 / 490-540$ & $1: 1000(38 \mathrm{mM})$ & Invitrogen; D3922 \\
\hline Nile Red & $488 / 490-620$ & $1: 1000(100 \mu \mathrm{g} / \mathrm{mL})$ & Invitrogen; N1142 \\
\hline LD540 & $514 / 520-570$ & $1: 250(2 \mu \mathrm{g} / \mathrm{mL})$ & $\begin{array}{l}\text { Gift from C. Thiele } \\
\text { (Spandl et al., 2009) }\end{array}$ \\
\hline LipidTOX DeepRed & $633 / 635-670$ & $1: 500(2 \mathrm{X})$ & Invitrogen; H34477 \\
\hline EGFP & $488 / 490-540$ & - & - \\
\hline mCherry & $561 / 570-712$ & - & - \\
\hline ECFP & $405 / 450-550$ & - & - \\
\hline EYFP & $514 / 515-552$ & - & - \\
\hline
\end{tabular}

\subsubsection{Lipid staining with Oil Red O}

In order to visualize storage lipids, the lysochrome dye Oil Red O was used. Fresh tissue samples were obtained from anaesthetized animals. Dissection was executed in $30 \%$ Glycerol/1xPBS on ice. Samples were fixated in 4\% Paraformaldehyde/1xPBS for $10 \mathrm{~min}$ (gentle shaking).

Afterwards, fixative was removed and samples were washed with equal volumes of 1xPBS (repeated three times, each washing step $5 \mathrm{~min}$ ). Then samples were 
permeabilized with $0.01 \%$ Digitonin in 1xPBS (20min, 30rpm). This was followed by three washing steps with equal volumes of 1 XPBS ( 5 min each step).

Staining solution was prepared freshly by mixing $6 \mathrm{~mL}$ of $0.1 \%$ Oil Red $\mathrm{O}$ in $100 \%$ Isopropanol (pelleted at $2500 \times \mathrm{g}, 4^{\circ} \mathrm{C}, 5 \mathrm{~min}$ prior use) with $4 \mathrm{~mL} \mathrm{H}_{2} \mathrm{O}\left(4^{\circ} \mathrm{C}\right)$. Staining solution was filtrated and warmed up to RT in a water bath. Samples were incubated in staining solution for $25 \mathrm{~min}$ (RT, 30rpm) followed by three washing steps with 1xPBS as described before. Finally, samples were mounted in 30\% Glycerol in 1xPBS and imaged.

Fixation and the lysochrome dyes lead to increased fusion of lipid droplets. Therefore, it is recommended to acquire images soon after sample preparation. Images were acquired with an Axiophot epifluorescence microscope (Zeiss) and a 40x Objective (Zeiss). Images were analysed in ImageJ v1.49m.

\subsubsection{Imaging of Malpighian tubules and gut-ring fat body}

In order to explant Malpighian tubules or gut-ring fat body, flies (from density seeding; 150 embryos/midsize vial) were raised for six days (kept in cohorts of 40 males; food changed every second day) upon eclosure. Flies were transferred into an empty vial and anesthetized by chilling on ice. For dissection, flies were grabbed at the thorax with the dorsal side directed upwards to the viewer. First the head was removed with forceps. In the next step, a fine tweezer was stabbed into the fly with one end between abdominal tergite $4-5$. Then the tweezer was closed to grab the cuticle and the whole abdomen was bend over into ventral direction in order to break up the abdomen. Then the posterior end was pulled away slowly along the anteroposterior axis. As the digestive tract should have been still connected to the anus it was possible to pull out the complete digestive system including the gut-ring fat body (in close distance to the transition from the mid- to the hindgut), posterior and anterior Malpighian tubule pairs and maybe even the crop. Tissues were mounted as described above with the added dyes, according to the caption of the figure. 


\subsubsection{Imaging of adult cuticle attached fat body}

For imaging of adult fat body tissue 6-day-old male flies (deriving from density seeding; 150 embryos/midsize vial; cohorts of 40 males after eclosure; food changed every second day) were dissected in 1X PBS. First flies were mechanically fixed by thrusting a preparation pin through the thorax into a silicone gel matrix with the ventral side facing upwards. Then the abdomen was sliced in transversal plane between abdominal tergite 6-7 using a fine scissor. Additional slices were performed in coronal plane along the tergital-sternital intersections. In order to expose the cuticle-attached fat body the ventral tissues (sternital parts, digestive and reproductive system, trachea) were removed. Finally, tissues were mounted. With its high hydrophobicity cuticle fat body samples had the tendency to flip in the small liquid volume on the slides. Therefore, instead of a microscope slide a cover slide (cover slide 22×22mm, VWR, Cat. \#: 631-0653) was used and samples were covered as described above. In order to ensure a proper and fast distribution of the dyes the carcass was flushed slowly with mounting medium using a pipette, then covered, and sealed with nail polish.

\subsubsection{Image acquisition}

If not stated otherwise, images were acquired with a Zeiss LSM710 microscope and a C-Apochromat 40x/1.20 W Korr. FCS M27 objective in 12-bit mode. Each image was adjusted for a dynamic signal range. Standard resolution was: $2000 \times 2000$ pixel mode, pixel acquisition time $12 \mu \mathrm{s} / \mathrm{pixel}, 70 \mathrm{~nm} /$ pixel (in ImageJ $13.48 \mathrm{pixel} / \mu \mathrm{m}$ ).

\subsubsection{Lipid Droplet Size quantification}

In order to quantify the size of lipid droplets the areas of lipid droplets were measured from single optical sections (tissues from different animals, multiple cells from one animal and various z-positions) for fat body tissue. In Malpighian tubules it was possible to acquire 3D-Z-stacks from various positions along the tubules (intersection of the tubules into the ureter, mid-segment and tip) and a maximum intensity projections were used for the analysis. Lipid droplet area quantification was performed in ImageJ v1.49m. 
A lipid droplet is defined by the area from the fluorescence signal that is distinguishable from the background due to accumulated lipophilic dye. Due to inhomogeneity of the lipid droplet composition and variable diffusion, lipid droplet staining normally suffers from strong differences in fluorescent signal intensities. Therefore, a good contrast (defined as the slope of the fluorescence intensity between background and a lipid particle) especially in the low-signal range is crucial for a successful detection of lipid droplets. For this, first the lookup table was changed to "HiLo" in ImageJ. Then the contrast was adjusted. The "Minimum" pixels were increased until the background becomes blue in the image. The exact values depend on the bit-depth of the picture and background intensity of the sample. Then a different lookup table was applied to the picture using an s-log curve to the picture (ImageJ macro available on request; macro was developed with Andres Hertel (MPI-BPC, Göttingen). Depending on the slope, signal intensities in the low-signal range were increased while higher intensity values were not modified (use the same slope-parameter for all analysed samples). By this, the contrast of lipid particles can be enhanced and intra-lipid droplet fluorescence signals become more homogenous. This enables a more reliable detection of lipid droplets in the following steps and ensures the detection of weaker stained lipid droplets (s-log curve should only be applied for applications that finally want to discriminate "signal-positive" areas. Signal intensities cannot be used during further analysis!).

In the next step, a "Gaussian blur" filter (2.0-pixel range, picture resolution: 13,5 pixel $/ \mu \mathrm{m}$ ) was applied to the picture to smooth out the edges of lipid droplets. Afterwards a FFT-Bandpass Filter (40 pixels, 3 pixels, none, 40\% settings in ImageJ) was applied to improve contrast of the lipid droplet fluorescence signal. A binary image was created by thresholding using "Moments B\&W" algorithm. Due to inhomogeneous intra-lipid droplet staining signal, thresholding may provide holes that were filled (Process->Binary->Fill holes). Clustered lipid droplets were separated by the "watershed" tool. Finally, the particle analyser was applied on the picture (size $\left(\mu \mathrm{m}^{2}\right)$ : 0.15 - Infinity; circularity: 0.01-1.0; exclude edges; show outlines) for area determination of discrete particles. Lipid droplet diameters and volumes were 
calculated assuming that lipid droplets are ideal spheres. Statistical analyses of lipid droplet size distributions were performed in OriginPro 9 using Mann-Whitney test.

\subsubsection{Electron Microscopy}

Ultrastructural analysis of lipid storage was performed in dissected Malpighian tubules from 6-day-old male flies (from density seeding; 150 embryos/midsize vial, cohorts of 40 males after eclosure; food changed every second day; $25^{\circ} \mathrm{C} ; 12 / 12 \mathrm{~h}$ light/dark-cycle, 50\% humidity). Flies were dissected in 1x PBS.

Samples were placed in a $150 \mu \mathrm{m}$ flat embedding specimen holder (Engineering Office M. Wohlwend $\mathrm{GmbH}$, Sennwald, Switzerland) and subsequently frozen in a Leica HBM high pressure freezer (Leica Microsystems, Wetzlar, Germany). Afterwards the vitrified samples were embedded using an Automatic Freeze Substitution Unit [AFS] (Leica).

Substitution was performed at $-90^{\circ} \mathrm{C}$ in: anhydrous acetone with $0.1 \%$ tannic acid and $0.5 \%$ glutaraldehyde for $72 \mathrm{~h}$. This was followed by an incubation in anhydrous acetone, $2 \% \mathrm{OsO} 4,0.5 \%$ glutaraldehyde for $8 \mathrm{~h}$ and additional $18 \mathrm{~h}$ at $-20^{\circ} \mathrm{C}$. Afterwards samples were warmed up to $+4^{\circ} \mathrm{C}$ and washed with anhydrous acetone. Further on, samples were embedded in Agar 100 (Epon 812 equivalent) at room temperature and polymerized for $24 \mathrm{~h}\left(60^{\circ} \mathrm{C}\right)$.

Images were acquired with a Philips CM120 electron microscope (Philips Inc.) equipped with a TemCam 224 A slow scan CCD camera (TVIPS, Gauting, Germany). Sample processing and image acquisition was done by Dr. Dietmar Riedel (Max Planck Institute for biophysical chemistry, facility for Transmission Electron Microscopy).

\subsubsection{Measurement of Mitochondrial diameter}

In electron microscope pictures mitochondria were measure with the ruler tool in Image $\mathrm{v} 1.49 \mathrm{~m}$. The maximal spread was measured for each discrete mitochondrion. Statistical analyses of lipid droplet size distributions were performed in OriginPro 9.1.0 using Mann-Whitney test. 


\subsection{Protein expression}

\subsubsection{Baculovirus-system}

The MultiBac system (Berger et al., 2004, Trowitzsch et al., 2010)was employed for recombinant protein expression. pFL-acceptor vector was modified (in this work) to have an 14His-tag and HRV3C-protease cleavage site $\mathrm{N}$-terminal of the expressed gene of interest. Genes were inserted into the 14His-HRV3C-pFL vector by Gibson assembly cloning (chemotransformation of $\mathrm{DH} 10 \alpha$ E. coli). Positive clones, the so-called expression constructs, were identified via colony PCR and verified by DNA sequencing. Expression constructs ( 100ng) were transformed (electroporation) into DH1OMultiBacY E. coli cells (gift from I. Berger). Clones were selected by antibiotics and blue/white selection (disrupted $\beta$-galactosidase-gene indicated a successful acceptor vector integration). Recombinant bacmids were isolated from a $5 \mathrm{~mL}$ overnight $\mathrm{LB}$-culture (with selective antibiotics, $37^{\circ} \mathrm{C}$, shaking). Cells were harvested at $4000 \times \mathrm{g}\left(4^{\circ} \mathrm{C}, 15 \mathrm{~min}\right)$ and resuspended in $250 \mu \mathrm{L}$ suspension buffer (ROCHE; Qiagen or Machenery-Nagel). $250 \mu \mathrm{L}$ of lysis-buffer were added and carefully mixed by inverting Eppendorf tubes several times. The mix was then incubated for $5 \mathrm{~min}$. After that $350 \mu \mathrm{L}$ neutralisation-buffer were added and mixed by inverting the tube several times. Precipitated protein was pelleted by centrifugation $\left(4000 \times \mathrm{g}, 10 \mathrm{~min}, 4^{\circ} \mathrm{C}\right)$. Supernatant was transferred to a fresh Eppendorf vial and pelleted again $\left(4000 \times \mathrm{g}, 10 \mathrm{~min}, 4^{\circ} \mathrm{C}\right)$. Supernatant was again transferred to a new Eppendorf vial and $700 \mu \mathrm{L}$ Isopropanol were added. Mix was incubated for $30 \mathrm{~min}$ at $-20^{\circ} \mathrm{C}$ and then pelleted at $17000 \mathrm{x}$ for $15 \mathrm{~min}\left(4^{\circ} \mathrm{C}\right)$. Liquid was removed and the DNA pellet was washed carefully with $70 \%$ Ethanol. After centrifugation (17000 x g, $10 \mathrm{~min}, 4^{\circ} \mathrm{C}$ ) liquid was removed and the pellet was washed with $30 \mu \mathrm{L} 100 \%$ Ethanol and again pelleted $\left(17000 \times \mathrm{g}, 10 \mathrm{~min}, 4^{\circ} \mathrm{C}\right)$. The DNA pellet was air-dried and finally $20 \mu \mathrm{L}$ of sterile $\mathrm{H}_{2} \mathrm{O}$ were added for resolubilization (no strong mixing by pipetting of vortexing).

Recombinant proteins were produced in insect cells as described earlier (Trowitzsch et al., 2010).

\subsubsection{Virus production and protein expression in Sf-9 cells}

Generated recombinant bacmids (see above) were transfected into Sf-9 cells (in Sf$900^{\mathrm{TM}}$ III SFM culture medium, $27^{\circ} \mathrm{C}$ ) for initial virus production in a 6 -well-plate format (use 1Mio cells/well) using Xtreme Gene transfection reagent (ROCHE, Cat. \#: 06365779001). After passaging and dilution cells should rest for at least $15 \mathrm{~min}$ prior transfection. 
$200 \mu \mathrm{L}$ of cell culture medium were added to the $20 \mu \mathrm{L}$ Bacmid preparation. Then $100 \mu \mathrm{L}$ culture medium were mixed with $10 \mu \mathrm{L}$ Xtreme Gene reagent and added to the $220 \mu \mathrm{L}$ Bacmid-cell culture medium solution. This was mixed by gently flipping of the vial and incubated for $1 \mathrm{~h}$ at $27^{\circ} \mathrm{C}$ (without movement; avoid strong light exposure). $150 \mu \mathrm{L}$ of the transfection mix were then used for one vial of a 6 -well-plate. For this the mix was expelled slowly from the tip and the single drops were distributed over the whole well homogenously.

Cells were incubated for $60-72 \mathrm{~h}$ at $27^{\circ} \mathrm{C}$. Virus production ( $\mathrm{V}_{0}$ virus) was monitored by GFP expression of the cells. After incubation time the $V_{0}$ virus (located in the medium) was used to inoculate a $25 \mathrm{~mL}$ suspension culture of SF-9 cells $\left(0,5^{\wedge} 10^{6}\right.$ cells $\left./ \mathrm{mL}, 27^{\circ} \mathrm{C}, 120 \mathrm{rpm}\right)$. Cell density and viability was measured every $24 \mathrm{~h}$ with a cell counter. Samples of $\sim 1$ Mio cells were collected and cell pellets (centrifugation at $10000 \times \mathrm{g}, 10 \mathrm{~min}, 4^{\circ} \mathrm{C}$ ) were stored for later analysis of protein expression. From the time point the cells stopped proliferation (Day of proliferation arrest; DPA) cells were incubated until DPA+60-72h to collect the $V_{1}$ virus (cell culture medium was collected in a $50 \mathrm{~mL}$ Falcon tube, pelleted $\left[4000 \times \mathrm{g}, 10 \mathrm{~min}, 4^{\circ} \mathrm{C}\right]$ and supernatant was sterile filtered $[20 \mu \mathrm{m}]$ ). $V_{1}$ was stored at $4^{\circ} \mathrm{C}$ (avoid light exposure of the virus; long-term storage at $-20^{\circ} \mathrm{C}$ possible but with lower $\mathrm{MOI}$ ).

\subsubsection{Protein expression in $\mathrm{Hi}-5$ cells}

In order to achieve a higher protein yield expression was performed in $\mathrm{Hi}-5$ cells (using Express Five ${ }^{\circledR}$ SFM culture medium, Life technologies, Cat. \#: 10486-025 supplemented with $20 \mathrm{~mL}$ L-Glutamine $(200 \mathrm{mM})$, Life technologies, Cat. \#: 25030032). $25-100 \mathrm{~mL}$ suspension cultures $\left(0,5^{\wedge} 10^{6}\right.$ cells $/ \mathrm{mL}$; $)$ were inoculated with various volumes of $V_{1}$ virus (virus volume should not exceed $2 \mu \mathrm{L} / \mathrm{mL}$ culture). Normally a $100 \mathrm{~mL}$ culture was inoculated with $100 \mu \mathrm{L} V_{1}$ virus. Cell viability and density were measured every $24 \mathrm{~h}$ and samples of 1 Mio cells were collected as described above. Cells were harvested when being in cell cycle arrest and a drop of the cell viability $\leq 70$ took place ( DPA $+48-72 \mathrm{~h}$ ). Normally, this was accompanied by a plateau in the GFP-intensity of the harvested samples. 


\subsubsection{E. coli expression system}

For protein expression of proteins cloned into the PASK-IBA5+ vector E.coli BL-21 were transformed with the respective vector (Table 3) as described recently (Nagy et al., 2014). Protein expression was induced at $\mathrm{OD}_{600 \mathrm{~nm}}=0.5-0.6$ by addition of $200 \mathrm{ng} / \mathrm{mL}$ anhydro-tetracycline (AHTC). Cultures were kept at $22^{\circ} \mathrm{C}$ for $3 \mathrm{~h}$ (180rpm, $300 \mathrm{~mL}$ ) until cells were harvested.

For bmm-GST E. coli BL-21 were transformed with the vector and protein expression was induced at $\mathrm{OD}_{600 \mathrm{~nm}}=0.5-0.6$ by addition of IPTG $(1 \mathrm{mM})$. Bacteria were cultured for $3 \mathrm{~h}$ at $22^{\circ} \mathrm{C}(180 \mathrm{rpm}, 300 \mathrm{~mL})$ until cells were harvested.

\subsubsection{COS-7 expression system}

Expression of proteins in COS-7 cells was performed as described principally (Zimmermann et al., 2004) with minor modifications (Schweiger et al., 2014). In detail, SV-40 transformed monkey kidney cells (COS-7, ATCC CRL-1651) were transfected with pCDNA4/HisMax vector coding for the protein of interest (Table 3). Cells were seeded one day before transfection $\left(9,5 * 10^{5}\right.$ cells $/ 10 \mathrm{~cm}$ Petri dish) and kept in DMEM (4,5g/L Glucose) with $10 \%$ foetal calf serum (FCS) and $5 \%$ antibiotics (penicillin, streptomycin) at $37^{\circ} \mathrm{C}\left(5 \% \mathrm{CO}_{2}\right)$.

Per transfection $300 \mu \mathrm{L}$ DMEM (serum-free) medium were mixed with $6 \mu \mathrm{g}$ Plasmid DNA. $27 \mu \mathrm{L}$ of Metafectene (Biontex $\mathrm{GmbH}$, Cat. \#: T020-1.0) were added to the solution and mixed. Afterwards the transfection mixture was incubated for $20 \mathrm{~min}$ at RT. For transfection standard culture medium was replaced by $4 \mathrm{~mL}$ serum- and antibiotic-free DMEM and transfection mixture was added to the cells. After incubation for $4 \mathrm{~h}$ the medium was changed back to $6 \mathrm{~mL}$ standard culture medium.

Cells were harvested after $48 \mathrm{~h}$ incubation. For this culture medium was removed and cells were washed with 1xPBS. Cells were mechanically removed (with a scraper) in $3 \mathrm{~mL} 1 \times$ PBS. Finally, cells were pelleted by centrifugation (3min, $1200 \times \mathrm{g}, \mathrm{RT}$ ) and 1XPBS was removed. 


\subsection{Lysate preparation for recombinantly expressed proteins}

Cell pellets were resuspended in $300 \mu \mathrm{L}$ lysis buffer $(250 \mathrm{mM}$ Sucrose, $1 \mathrm{mM}$ EDTA, 1mM DTT, 1x Protease inhibitor [ROCHE; Cat. \#: 04693116001]) and kept on ice. Disruption of cells was done by sonication (Branson; S-450A with a micro-tip). In order to remove cellular debris, the lysate was pelleted $\left(1000 \times \mathrm{g}, 4^{\circ} \mathrm{C}, 15 \mathrm{~min}\right)$. The supernatant was collected and stored at $-20^{\circ} \mathrm{C}$ for further use. Protein expression was assayed by Western blot and protein concentration was determined by Bradford protein assay according to the manufacturer (ThermoFisher scientific, Cat. \#:23200). Finally, substrate concentration was adjusted to $2 \mathrm{mg}$ protein/ $\mathrm{mL}$ using lysis buffer.

\subsection{Western Blot and Immunohistochemistry}

In order to detect proteins a western blot was performed. Protein samples were dissolved in sample buffer (final $1 x$; Stock $3 x$ : 150mM Tris [pH 6.8], 6\% SDS, 0.3\% Bromophenol blue, $30 \%$ Glycerol, $300 \mathrm{mM}$ DTT). Sample mix was then incubated for $3 \mathrm{~min}$ in a water bath $\left(95^{\circ} \mathrm{C}\right)$ and stored back on ice. Protein size separation was performed by SDS-PAGE (12\%).

Afterwards samples were transferred onto a nitrocellulose membrane (Thermo scientific, Cat. \#: 80018 ) by electro-blotting (20W, $2 \mathrm{~h}, 4^{\circ} \mathrm{C}$ ). Afterwards, the blots (membrane with transferred protein on it) were washed in 1 XTBST (20mM Tris pH $7.5,150 \mathrm{mM} \mathrm{NaCl}, 0.1 \%$ Tween20). In order to reduce unspecific binding of the used primary antibodies the blots were blocked with 1xTBST+5\%BSA (2h, RT, 30rpm).

Then blots were incubated with the primary antibodies (used concentrations in the table) in 1xTBST+5\%BSA for $1 \mathrm{~h}$ (RT, $30 \mathrm{rpm}$ ). After washing with 1xTBST blots were incubated with secondary antibodies (30 min, RT, 30rpm). In order to remove unbound secondary antibody that provide unspecific- and background signal blots were washed another time with 1xTBST.

For detection of HRP-conjugated secondary antibody an luminol-based enhanced chemiluminescent substrate was used (Super Signal West Pico Chemiluminescent Substrate; Cat. \#: 34080). Membrane was incubated with working solution for $5 \mathrm{~min}$ (RT, $100 \mu \mathrm{L} / \mathrm{cm}^{2}$ membrane). Excess liquid was removed and membrane was placed 
between to plastic foils to prevent drying. Finally, chemiluminescence was detected with a Fujjifilm LAS-1000 CH Plus CCD Camera System. Images were analysed with ImageJ v1.49m.

\section{Table 6 Antibodies}

\begin{tabular}{|c|c|c|c|}
\hline $\begin{array}{l}\text { Name / } \\
\text { Antigen }\end{array}$ & Host; Subclass & $\begin{array}{c}\text { used } \\
\text { concentration }\end{array}$ & Supplier \\
\hline$\beta$-tubulin & mouse; IgG1 & $1: 5000$ & Hybridoma Bank \#E7 \\
\hline $\begin{array}{c}\alpha \text {-mouse lgG- } \\
\text { HRP }\end{array}$ & goat & 1:1000 & $\begin{array}{c}\text { Pierce, Cat. \#: } 31430 \text {, used } \\
\text { with } \beta \text {-tubulin }\end{array}$ \\
\hline$\alpha$-His & rabbit & 1:10000 & gift from Görlich department \\
\hline$\alpha$-rabbit-HRP & goat & $1: 12000$ & Pierce, Cat. \#: 31460 \\
\hline$\alpha-G F P(S y S y)$ & rabbit & 1:7000 & $\begin{array}{c}\text { Synaptic Systems, Cat. } \\
\#: 132002\end{array}$ \\
\hline a_Strep-tag II & mouse, IgG & 1:5000 & IBA, Göttingen \\
\hline $\begin{array}{c}\alpha \text {-mouse lgG- } \\
\text { HRP }\end{array}$ & sheep & 1:10000 & $\begin{array}{l}\text { GE Healthcare Amersham; } \\
\text { used with } \alpha \text { Strep-tag II }\end{array}$ \\
\hline
\end{tabular}

\subsection{Immunohistochemistry in larvae}

Larval were dissected in phosphate buffer saline (PBS) for analysis of subcellular localization of proteins. Isolated fat bodies were carefully washed with PBS and fixed in $4 \%$ formaldehyde for 15-20 minutes at room temperature. After fixation fat bodies were washed with BBT solution (10 mM Tris, $55 \mathrm{mM} \mathrm{NaCl}, 40 \mathrm{mM} \mathrm{KCl}, 7 \mathrm{mM} \mathrm{MgCl}$, $20 \mathrm{mM}$ glucose, $50 \mathrm{mM}$ sucrose, $\mathrm{pH} 7.0$ and $0.1 \%$ Tween-20) and $0.2 \%$ BSA. Afterwards samples were blocked in 3\% (v/v) horse serum (diluted in BBT) for 2 hours at room temperature. Than fat bodies were incubated with the primary antibody over night at $4^{\circ} \mathrm{C}$. After washing with BBT the samples were incubated with the secondary antibody in 1:500 dilution for 2 hours at room temperature. In the last step fat body was mounted with DAPI and LipidTox as described earlier (2.6.1) and images were acquired.

\subsection{Enzymatic assays}

\subsubsection{Triglyceride hydrolase assay}

Hydrolase activity of proteins on triglyceride was measured as described in (Schweiger et al., 2014) based on a protocol from Holm et al. (Holm et al., 2001). 
For the assay needed amounts for finally $1.67 \mathrm{mM}$ Triolein (Sigma-Aldrich, Cat. \#: T7140 ) were dosed with $10 \mu \mathrm{Ci} / \mathrm{mL}^{3} \mathrm{H}$-Triolein $([9,10(\mathrm{~N})-3 \mathrm{H}]$-Triolein, Perkin Elmer, Cat.

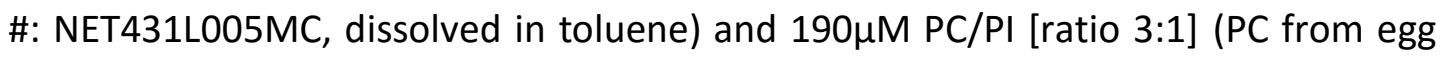
yolk and PI [Cat. \#: P-3556] from soybean [Cat. \#: P-0639], Sigma Aldrich, dissolved in Chloroform) were added to a reaction tube. The solvents were evaporated under a stream of nitrogen $(\mathrm{g})$.

$2 \mathrm{~mL}$ of $0.1 \mathrm{M}$ potassium phosphate buffer (KPB; $\mathrm{pH} 7.0$ ) were added initially and sonicated (30s, $20 \%$ output power, on ice). Ideally, the substrate mix should become turbid. Then the remaining KPB was added to get the final concentrations mentioned above (keep in mind that BSA still needs to be added). Samples were then sonicated again (15s, 20\% output power, on ice). Afterwards the fatty acid (FA) acceptor fatfree BSA (20\% stock solution in KPB, Sigma Aldrich, Cat. \#: A6003) was added to a final concentration of $5 \%$. One aliquot of substrate was measured to determine the specific substrate activity (should be $\sim 1 \times 10^{6} \mathrm{dpm} / 100 \mu \mathrm{L}$ ).

For hydrolysis assay $100 \mu \mathrm{L}$ sample $(2 \mathrm{mg}$ protein $/ \mathrm{mL})$ were mixed with $100 \mu \mathrm{L}$ substrate. As blank $100 \mu \mathrm{L}$ substrate were mixed with $100 \mu \mathrm{L}$ lysis buffer. Protein amounts for the assay need to be optimized in order to be in the linear range of the assay. Samples were tested at least as triplicates. The reaction mix was then incubated in a water bath $\left(1 \mathrm{~h}, 37^{\circ} \mathrm{C}\right.$, continuous shaking). The reaction was terminated by the addition of $3.25 \mathrm{~mL}$ Methanol: Chloroform: $\mathrm{n}$-heptane (10:9:7 $\mathrm{v} / \mathrm{v} / \mathrm{v})$. Then $1.05 \mathrm{~mL}$ of $0.1 \mathrm{M}$ potassium carbonate $(\mathrm{pH} 10.5$ [adjusted with saturated Boric acid) were added and mixed vigorously for $5 \mathrm{~s}$ by vortexing. Phase separation was achieved by centrifugation (1000 x g, 10min, RT). $200 \mu \mathrm{L}$ of the aqueous (upper one) phase were mixed with $2 \mathrm{~mL}$ of scintillation cocktail and radiometrically analysed. Statistical analysis was performed in OriginPro 9.1.0 as described in the figure caption.

The rate of Triglyceride hydrolase activity is represented as:

$$
\frac{\text { nmol }_{\text {released fatty acid }}}{h \times m g \text { protein }}
$$


A partition coefficient of 1.9 (71,5\% recovery) for the extraction of released fatty acids into aqueous phase was used (Schweiger et al., 2014). For the calculation of the activity rate following equation was applied:

$$
\frac{n m o l_{\text {released fatty acid }}}{h \times m g \text { protein }}=\frac{(d p m \text { sample }-d p m B L A N K) \times\left(\frac{V_{\text {total aqueous phase }}}{V_{\text {used for scintillation counting }}}\right)}{\left(\frac{d p m \text { substrate }}{n m \text { flatty }_{\text {facids }}}\right)^{1} \times m g \text { protein } \times 0,715 \times t_{\text {incubation }}}
$$

For $1,67 \mathrm{mM}$ Triolein $\mathrm{n}_{\text {fatty acids }}=501 \mathrm{nmol}$ fatty acids $/ 100 \mu \mathrm{L}$ substrate were used.

\subsubsection{Hitfinder assay}

In order to identify possible substrates a screen testing different neutral and phospholipids was used.

In reaction volume of $25 \mu \mathrm{L}$ different substrates (see table) were tested at $1 \mathrm{mM}$ concentration and released fatty acids were measured colorimetrically with the NEFA HR(2)-Kit (Wako Chemicals, Cat. \#: 434-91795 and 436-91995). Samples lysates were prepared as described before. The $2 \mathrm{mg} / \mathrm{mL}$ samples were diluted 1:3 in Assay buffer $(2,5 \mathrm{mM}$ EDTA, $250 \mathrm{mM} \mathrm{KCl}, 12,5 \mathrm{mM}$ CHAPS and finally $5 \% \mathrm{BSA})$ and $15 \mu \mathrm{L}$ were used for a reaction. Samples were run as duplicates in one well of a 96 -well plate.

Substrates were prepared as a stock-solution for the assay. Needed amounts (prepare $400 \mu \mathrm{L}$ with $2.5 \mathrm{mM}$ ) of substrate were placed in a $1.5 \mathrm{~mL}$ Eppendorf tube and organic solvent was evaporated under a stream of nitrogen. Then substrate was dissolved in $300 \mu \mathrm{L}$ assay buffer and sonicated ( $20 \%$ output power, $2 \times 20$ s, on ice). Then $100 \mu \mathrm{L}$ of $20 \%$ of fat-free BSA (in Assay buffer, Sigma Aldrich, Cat. \#: A6003) were added as fatty acid acceptor and mixed thoroughly.

$10 \mu \mathrm{L}$ of substrate-solution were added to the $15 \mu \mathrm{L}$ of prepare sample and incubated (30min, $37^{\circ} \mathrm{C}, 120 \mathrm{rpm}$ ). Plate should be covered to avoid evaporation. Released fatty acids were measured by the NEFA assay (described before). 


\subsubsection{List of substrates}

\section{Table 7 Substrates for Enzymatic assays}

\begin{tabular}{|c|c|c|}
\hline Symbol & Substance & Manufacturer \\
\hline PC & 1,2-dioleoyl-sn-glycero-3-phosphocoline & Sigma Aldrich \\
\hline PA & 1,2-dioleoyl-sn-glycero-3-phosphate (sodium salt) & Enzo Life Sciences \\
\hline PG & 1,2-dioleoyl-sn-glycero-3-phospho-(1'-rac-glycerol) (sodium salt) & Sigma Aldrich \\
\hline NAPE & $\begin{array}{l}\text { 1,2-dioleoyl-sn-glycero-3-phosphoethanolamine- } \mathrm{N} \text {-arachidonoyl } \\
\text { (ammonium salt) }\end{array}$ & Enzo Life Sciences \\
\hline MCPG & $\begin{array}{l}\text { 1,2-dioctanoyl-sn-glycero-3-phospho-(1'-rac-glycerol) (sodium } \\
\text { salt) }\end{array}$ & Sigma Aldrich \\
\hline MCPC & 1,2-dioctanoyl-sn-glycero-3-phosphocholine & Sigma Aldrich \\
\hline$B M P(R, R)$ & $\begin{array}{l}\text { sn-(3-oleoyl-2-hydroxy)-glycerol-1-phospho-sn-1'-(3'-oleoyl-2'- } \\
\text { hydroxy)-glycerol (ammonium salt) }\end{array}$ & Avanti Polar Lipids \\
\hline LPC & 1-oleoyl-2-hydroxy-sn-glycero-3-phosphocholine & Sigma Aldrich \\
\hline LPA & 1-oleoyl-2-hydroxy-sn-glycero-3-phosphate & Avanti Polar Lipids \\
\hline LPG & $\begin{array}{l}\text { 1-oleoyl-2-hydroxy-sn-glycero-3-phospho-(1'-rac-glycerol) } \\
\text { (sodium salt) }\end{array}$ & Sigma Aldrich \\
\hline MO & 1-(9Z-octadecenoyl)-rac-glycerol & in Stock \\
\hline Cardiolipin & 1,3-bis(sn-3'-phosphatidyl)-sn-glycerol (in bovine heart mainly C18:2) & Sigma Aldrich \\
\hline pNPB & para-Nitrophenylbutyrate & Sigma Aldrich \\
\hline PE & 1,2-dioleoyl-sn-glycero-3-phosphoethanolamine & Sigma Aldrich \\
\hline PS & 1,2-dioleoyl-sn-glycero-3-phospho-L-serine (sodium salt) & Sigma Aldrich \\
\hline PI & 1,2-dioleoyl-sn-glycero-3-phospho-(1'-myo-inositol) (ammonium salt) & Sigma Aldrich \\
\hline CL & 1`,3`-bis[1,2-dioleoyl-sn-glycero-3-phospho]-sn-glycerol (sodium salt) & Sigma Aldrich \\
\hline $\mathrm{BMP}(\mathrm{S}, \mathrm{s})$ & $\begin{array}{l}\text { sn-(3-oleoyl-2-hydroxy)-glycerol-1-phospho-sn-1'-(3'-oleoyl-2'- } \\
\text { hydroxy)-glycerol (ammonium salt) }\end{array}$ & Avanti Polar Lipids \\
\hline LPS & 1-oleoyl-2-hydroxy-sn-glycero-3-phospho-L-serine (sodium salt) & Sigma Aldrich \\
\hline LPE & 1-oleoyl-2-hydroxy-sn-glycero-3-phosphoethanolamine & Sigma Aldrich \\
\hline LPI & L- $\alpha$-lysophosphatidylinositol (Liver, Bovine) (sodium salt) & Sigma Aldrich \\
\hline MP & Methylpalmitate & Sigma Aldrich \\
\hline EP & Ethylpalmitate & Sigma Aldrich \\
\hline PP & 2-propyl palmitate & Sigma Aldrich \\
\hline BP & 1-Butyl palmitate & Sigma Aldrich \\
\hline DO (rac) & 1,2-dioleoyl-sn-glycerol & Sigma Aldrich \\
\hline TO-C18:1 & 1,2,3-(9Z-octadecenoyl)-glycerol & Sigma Aldrich \\
\hline TO-C8 & Glyeroltrioctanoate & Sigma Aldrich \\
\hline
\end{tabular}




\begin{tabular}{|cll|}
\hline MGDG & 1,2-diacyl-3-O- $\beta$-D-galactosyl-sn-glycerol & Sigma Aldrich \\
\hline RE & Retinylpalmitate & Sigma Aldrich \\
\hline CO & Cholest-5-en-3 $\beta$-yl octadecanoate & Sigma Aldrich \\
\hline WE & Arachidyl laurate & Sigma Aldrich \\
\hline PlasmaPC & 1-(12-octadecenyl)-2-oleoyl-sn-glycero-3-phosphocholine & Sigma Aldrich \\
\hline BDP (S,S) & sn-[2,3-dioleoyl]-glycerol-1-phospho-sn-1' '-[2',3'-dioleoyl]- & Sigma Aldrich \\
& glycerol (ammonium salt) & \\
\hline PMG & 1-O-hexadecyl-2-O-methyl-sn-glycerol & Sigma Aldrich \\
\hline O-AC-Cer & 1-oleoyl-N-heptadecanoyl-D-erythro-sphingosine & Sigma Aldrich \\
\hline
\end{tabular}

\subsubsection{Kinetics and analysis}

Michaelis constant $\left(\mathrm{K}_{M}\right)$ and maximal reaction rate $\left(\mathrm{V}_{\max }\right)$ were analysed using the reaction parameter from the Hitfinder Assay. First, the reaction conditions from the Hitfinder Assay were used and constant sample concentrations were incubated with the substrate $(2 \mathrm{mM})$ for various times (reaction volume scaled up to $50 \mu \mathrm{L}$ ). The longest time span that showed a linear conversion rate of the substrate into product + fatty acids (detected with the NEFA-Assay) was used for the further analysis. Various substrate concentrations $(0.25,0.5,0.75,1,1.25,1.5,1.75,2 \mathrm{mM})$ were tested for selected substrates of the Hitfinder assay. For each concentration, the fatty acid release was measured as duplicates. Assuming that PUML follows a MichaelisMenten kinetic the quantified amounts of fatty acids / time (constant time) released at the various substrate conditions were plotted against the concentration. The reaction rate can be described by the Michaelis-Menten equation:

$$
\text { reaction rate }(v)=\frac{d[P]}{d t}=\frac{V_{\max }[S]}{K_{M}+[S]}
$$

Based on the empirical values for the reaction rate $(v), V_{\max }$ and $K_{M}$ were calculated by non-linear regression using the Solver Add-in of Excel (Microsoft Office 2013) by minimizing the sum of the squared normalized errors for the measured $v$ at the various substrate concentrations $[\mathrm{S}]$ :

$$
\text { normalized } \text { error }^{2}=\left(\frac{v_{\text {estimated }}-v_{\text {measured }}}{v_{\text {measured }}}\right)^{2}
$$




\section{Results}

\subsection{Body fat storage in pummelig mutant flies}

In mammals the adipose triglyceride lipase (ATGL) is activated by $\alpha / \beta$-hydrolase domain containing 5 (ABHD5). In the Drosophila fat body Brummer lipase (DmATGL) is crucial for storage lipid mobilization and a knock out leads to increased amounts of body fat. With the high sequence similarity of Puml to mammalian ABHD5 it was assumed that this activation mechanism might be evolutionary conserved. With a possible interaction it was checked if may obtain comparable phenotypes when we interfere with the genes (overexpression or knock out/down) as both genes are expressed in the fat body (Gelbart and Emmert, 2013).

\subsubsection{Lipid storage is increased in pummelig mutants}

Body fat storage of pum/ ${ }^{1}$ flies was quantified by previous contributors to the pumlproject but a modulation of body fat storage could not be observed (Takacs, 2007, Rosenberg, 2012). Thus, to eliminate effects that could arise from different genetic backgrounds a genetically matched control strain was generated to the pum/ ${ }^{1}$ mutant strain (Rosenberg, 2012). In this work, it was shown that the absence of puml leads to a significant increase in the amount of storage body fat comparable to effect upon loss off bmm (Grönke et al., 2005) in adult flies analysed by a glyceride based colorimetric assay (CCA) (see Figure 13). The combination of the puml-allele with a deficiency-allele covering the puml gene locus supports the exclusive effect of the pum/ gene on body fat storage. Similar to a knockdown of $\mathrm{bmm}$, body fat storage can be increased by a knockdown of puml specifically in the fat body tissue (FB$S N S>G A L 4)$. 


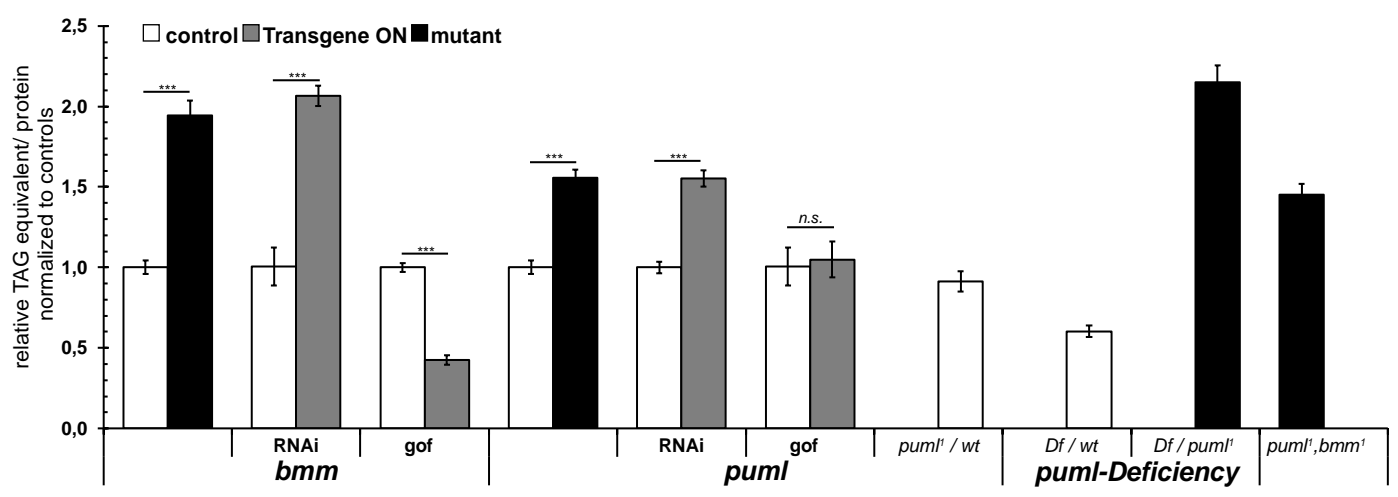

Figure 13 Interference with bmm and puml can lead to changes in body fat measure by CCA assay. Adiposity in response to RNAi-mediated gene knockdown is compared with null mutants. Additionally, crosses of puml ${ }^{1}$ with deficiency (Df) lines were analysed as well as gain-of-function (gof) experiments. Downregulation/absence of triglyceride lipase Brummer (BMM) leads increased body fat storage; detected by CCA assay. A knock out (puml ${ }^{1}$ or Df) or downregulation of puml by RNAi leads to increased amounts of body fat. In contrast to bmm-gain function (gof), which leads to lean flies, no effect can be detected in puml-gof flies. A puml ${ }^{1}$ bmm $^{1}$ double mutant reveals no additive effect on the body fat storage level of the two mutant alleles. Means of relative TAG equivalents/protein normalized to average TAG equivalents/protein values of the corresponding controls \pm SEM are shown; MannWhitney test, ${ }^{* *}=P<0.001 ;$ RNAi and gof were performed in the fat body (FB-SNS $>$ GAL4).

A gain-of-function of $\mathrm{bmm}$ in the fat body (FB-SNS>GAL4) leads to lower body fat storage ((Grönke et al., 2005),Figure 13). This cannot be observed during a gain-offunction of puml. A double knockout of $\mathrm{bmm}$ and pum/ has no additive effect on body fat storage and did not exceed amounts of pum/ ${ }^{1}$ flies. In addition to the CCA assay, a thin layer chromatography was performed to distinguish which neutral lipid class was changed (TAG, DAG or MAG).

Comparable to $\mathrm{bmm}^{1}$ flies (Grönke et al., 2005) also pum/ ${ }^{1}$ flies exhibited increased amounts of Triacyglycerides (TAGs) (Figure 14). Additionally, a trend for elevated amounts of DAGs was visible but no significant. The amount of FA was slightly increased (+27\%) in $\mathrm{bmm}^{1}$, but the low general abundance of FAs results in a very small effect size $(<1 \mu \mathrm{g})$ FAs between control flies and $\mathrm{bmm}^{1}$. 

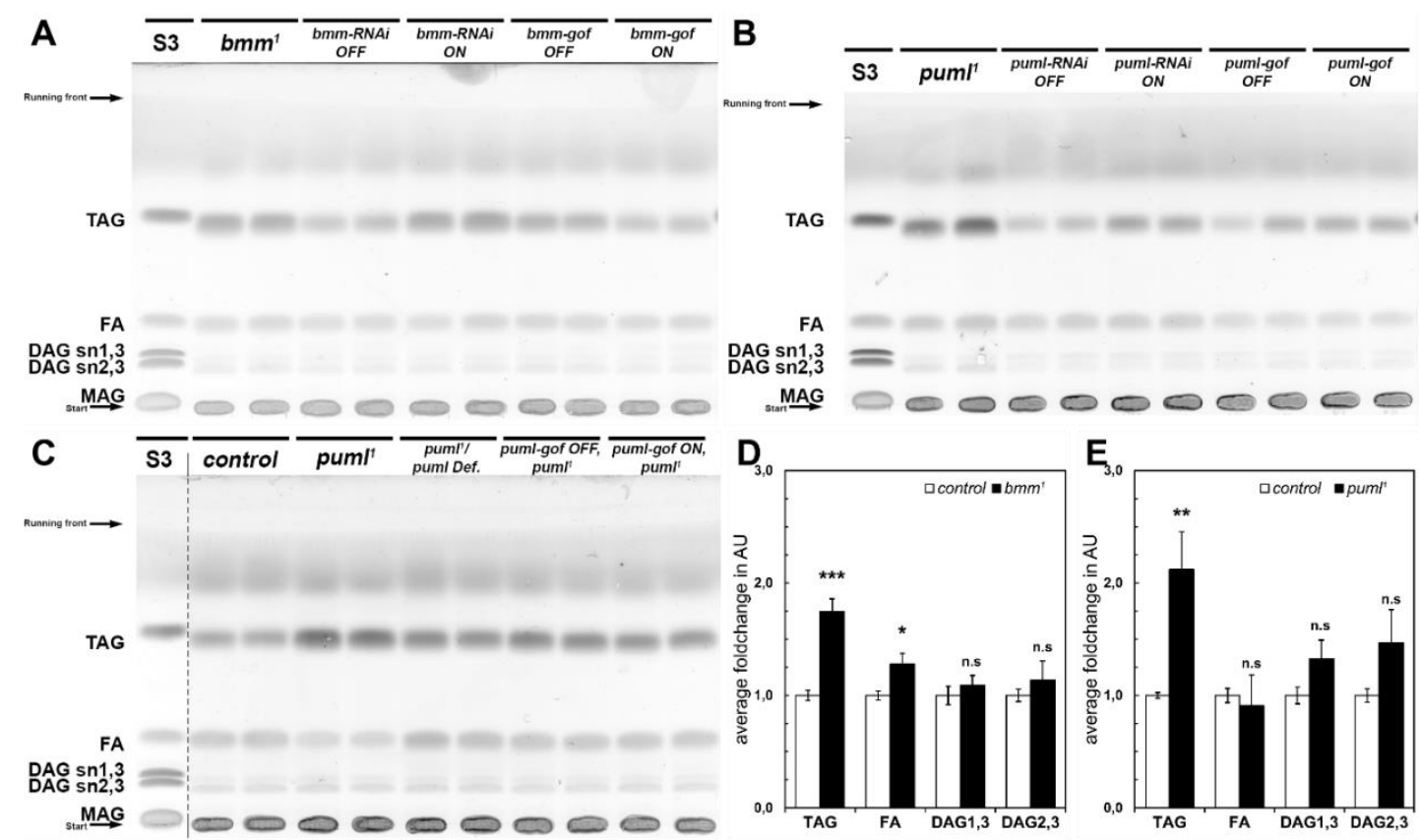

Figure 14 Amounts of Triacylglerides are increased in knock out mutants of puml and bmm. A thin layer chromatography analysis $(A, B, C)$ identifies amount of TAGs being significantly $(E, D)$ higher in $\mathrm{bmm}^{1}$ and puml ${ }^{1}$ flies. Loaded amounts of total lipids for a single TLC lane were normalized by protein to the equivalent of one control fly per lane. Plotted are the means of average fold changes in Arbitrary units (AU; measured densiometrically using ImageJ v1.49m) for the annotated lipid classes compared to control flies $\pm S E M$; Student's $t$-test, ${ }^{* * *}=P<0.001,{ }^{* *}=P<0.01, *=P<0.05$. In $C$ the dotted line marks a break due to a removal of a lane on the TLC plate.

\subsection{2 pummelig and brummer mutants are obese but not overweight}

In addition to body fat measurements it was tested if puml ${ }^{1}$ and $\mathrm{bmm}^{1}$ flies are overweight. Indeed, both mutants were actually lighter than control flies (Figure 15). pum $I^{1}$ had $\sim 15 \%$ decrease in body weight whereas the effect was less pronounced in $b m m^{1}(-8 \%)$. This indicates that obesity in flies does not necessarily correlate with overweight in flies.

\subsubsection{Body fat over-storage can be observed in pummelig mutant flies but not} larvae

So far it could be shown that adult puml $l^{1}$ flies store more fat than control flies but I was interested if this phenotype can be also observed in larvae. Additional body fat measurements revealed no significant changes in L3 larvae. A first difference in total body fat storage between pum/ ${ }^{1}$ and controls could be observed in freshly eclosed flies that became even more pronounced in older flies (Figure 15). As the adult fat body develops within the first six days after eclosure it might be possible that the 
differences in the regulation of the larval and the adult fat body might be reason that only in adult pum $l^{1}$ flies stored more fat than control flies.

A

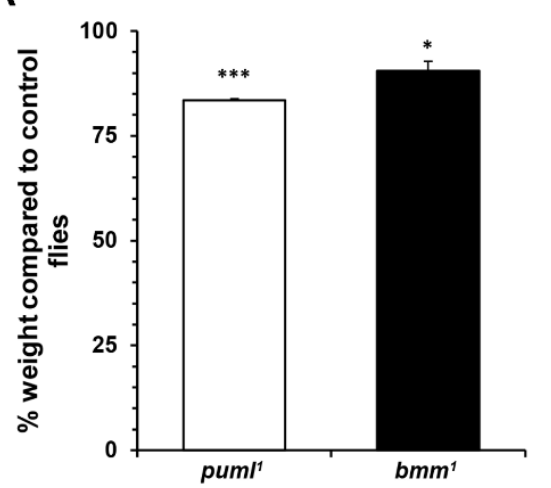

B

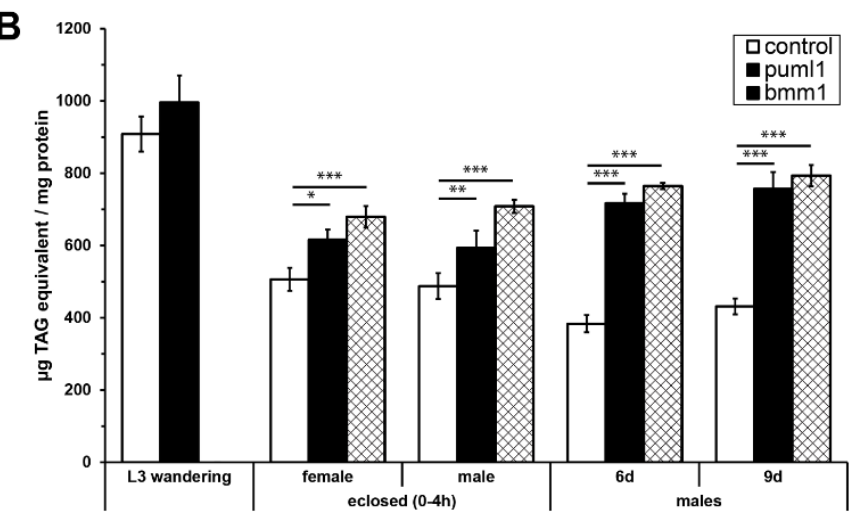

Figure 15 pummelig mutant flies are obese but not larvae. (A) Body weight of pum/ ${ }^{1}$ and bmm ${ }^{1}$ flies was decreased significantly compared to control flies. (B) Increased lipid storage of pum $l^{1}$ mutants can be observed in freshly eclosed flies (one-way ANOVA $F 1,14=0.04, P=0.02$ ) but not in wandering $L 3$ larval stage (one-way ANOVA $F 1,14=0.04, P=0.82$ ). There was no difference between freshly eclosed females or males (two-way ANOVA F1,34=0.55, $P=0.46$ ).

\subsection{Mean life time is decreased in pummelig mutant flies}

Lipid overstoring flies like fly mutants of the insulin pathway have an expanded lifespan (Partridge et al., 2011). On the other hand, $b \mathrm{~mm}^{1}$ that have higher body fat as well have a shorter lifespan (Grönke, 2005). Compared to its genetically matched control, pum/ ${ }^{1}$ flies had a significantly shorter mean life expectancy of $\sim 12 \%$ (Figure 16). In the two independent experiments the difference was significant. Whereas the puml ${ }^{1}$ strain had a very similar death curve and an average median lifetime of $62,6 \pm 0,9$ days, the control flies had a bigger variation $70,2 \pm 2,5$ days. Thus, a knockout of puml negatively affects the life expectancy in flies.
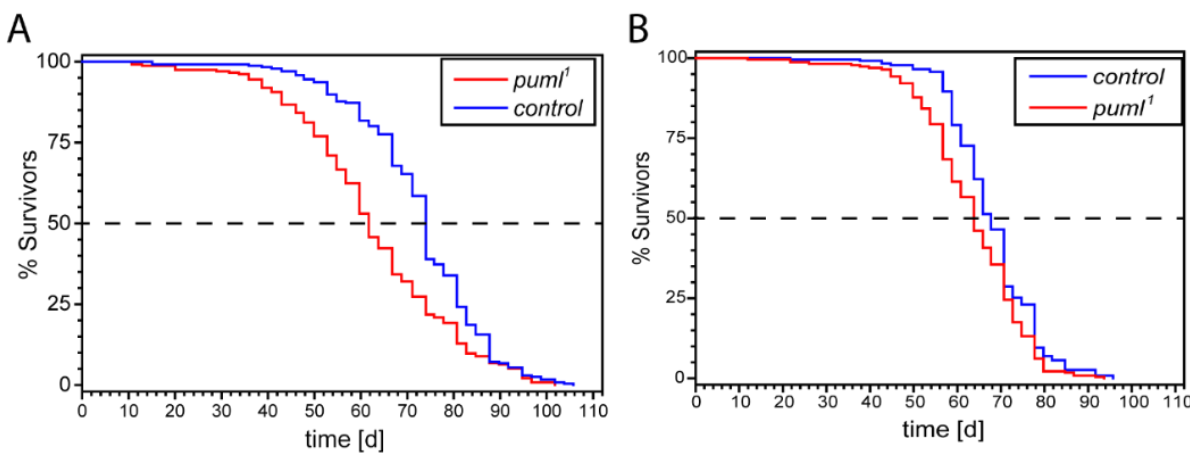

Figure 16 Mean-life time is decreased in pum/ ${ }^{1}$ compared to genetically matched control flies $(A, B)$. The two repetitions show the bigger variation in median life time of the control flies (log Rank-P test; $n=240$ animals/genotype; $A$ ) $P=3.47^{*} 10^{-8}$; B) $P=2.9 * 10^{-4}$ ). 


\subsection{Pummelig a starvation-responsive gene}

\subsubsection{Expression of pummelig is increased under starvation}

It is known that $b m m / D m A T G L$ is a starvation responsive gene (Grönke et al., 2005) and over-expression of $\mathrm{bmm}$ leads to reduced amounts of body fat (Grönke et al., 2005). As described earlier pum/ ${ }^{1}$ flies exhibit a comparable lipid over-storage phenotype to $\mathrm{bmm}^{1}$ flies (Figure 13). Additionally, Puml has high similarity to the activator of mammalian triacylglyceride lipase (ATGL) namely ABHD5/CGI-58 (Figure 10). Therefore, it was assumed that the core lipid mobilization module ATGL-ABHD5 could be evolutionary conserved in Drosophila melanogaster and that higher expression of $\mathrm{bmm}$ might be correlated with higher expression of puml and increased amounts of Puml.

In order to address this, $b m m$ expression was modulated by starvation (for $16 \mathrm{~h}$ ) or fat-body specific overexpression (FB-SNS>GAL4) and relative RNA levels of pum/ were analysed by RT qPCR (RNA isolated from total flies). Indeed, like $\mathrm{bmm}(19 \pm 5.8$ fold) also puml (Figure 17) showed a significant increase $(3 \pm 1,5$ fold) in RNA levels under starvation and was elevated $(9,3 \pm 3$ fold) while $b m m$ was over-expressed in the fat body (Figure 17). In order to analyse whether protein levels of Puml were elevated as well, a starvation experiment was performed using flies with puml::gfp under its endogenous promotor and compared to fed flies from the same strain. After $16 \mathrm{~h}$ flies from both groups were collected and Puml::GFP abundance was checked by Western Blot analysis using whole fly extracts. Comparable to the RNA levels, abundance of Puml::GFP was significantly increased by 4 fold during starvation (Figure 17). The data indicate an involvement of puml in the modulation of lipid mobilization.

A

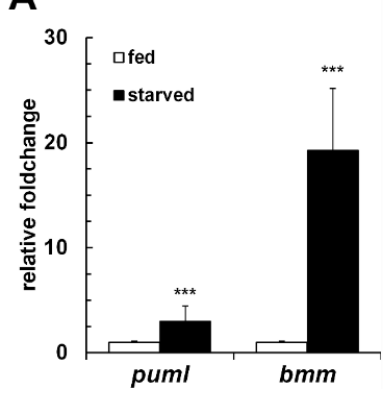

B

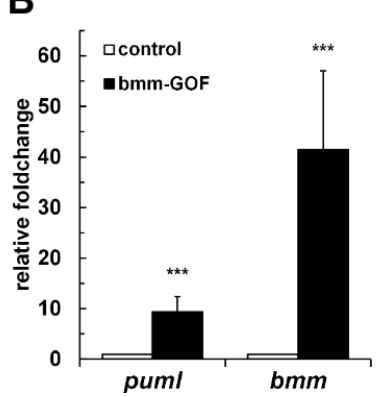

C

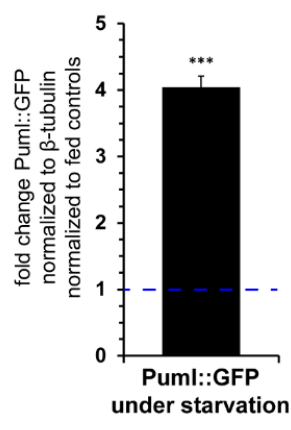

Figure 17 puml and bmm RNA levels are increased under starvation. Puml::GFP protein amounts (expressed under the endogenous promotor) are elevated under starvation. (A) Starvation leads to 
increased amounts of puml and bmm RNA. (B) Overexpression of bmm specifically in the fat body (FBSNS>GAL4) enhances RNA levels of puml as well (shown are the average fold changes \pm SEM of the RNA levels; Mann-Whitney test; $\left.{ }^{* * *}=P<0.001\right)$. (C) Detection of endogenously expressed Puml::GFP using $\alpha$-GFP antibody during starvation shows increased protein abundance of Puml::GFP. Plotted are means of Puml::GFP signal measured densiometrically normalized to $\beta$-tubulin measured on the same western blot membrane \pm SEM, Mann-Whitney test; $\left.{ }^{* * *}=P<0.001\right)$.

\subsubsection{Starvation resistance is enhanced in pummelig mutant flies}

As shown before both $\mathrm{bmm}^{1}$ as well as pum/ ${ }^{1}$ flies have increased lipid storage. Also, bmm and puml have higher expression during starvation. It is known from literature that, the lipid over-storage phenotype of $b m m^{1}$ flies is accompanied by a higher starvation resistance (Grönke et al., 2005). Therefore, it was checked if this is also the case for pum/ ${ }^{1}$ flies. Earlier experiments (Takacs, 2007) indicated no phenotype but the outbred puml ${ }^{1}$ strain used in this work exhibits a significant increase in the median starvation time (Figure 18). Compared to control flies which had a mean survival time of $38,5 \pm 4,2$ hours (average from five independent experiments) it was increased by $15 \%$ in puml ${ }^{1}$ flies $\left(44,7 \pm 3,8\right.$ hours). $b m m^{1}$ flies exhibited the biggest difference in mortality under food-deprivation with an increase of $56 \%$ in the mean survival time $\left(60,1 \pm 2,5\right.$ hours). Thus, the higher body fat storage in $\mathrm{bmm}^{1}$ and pum/ ${ }^{1}$ allows for a higher survival under starvation.
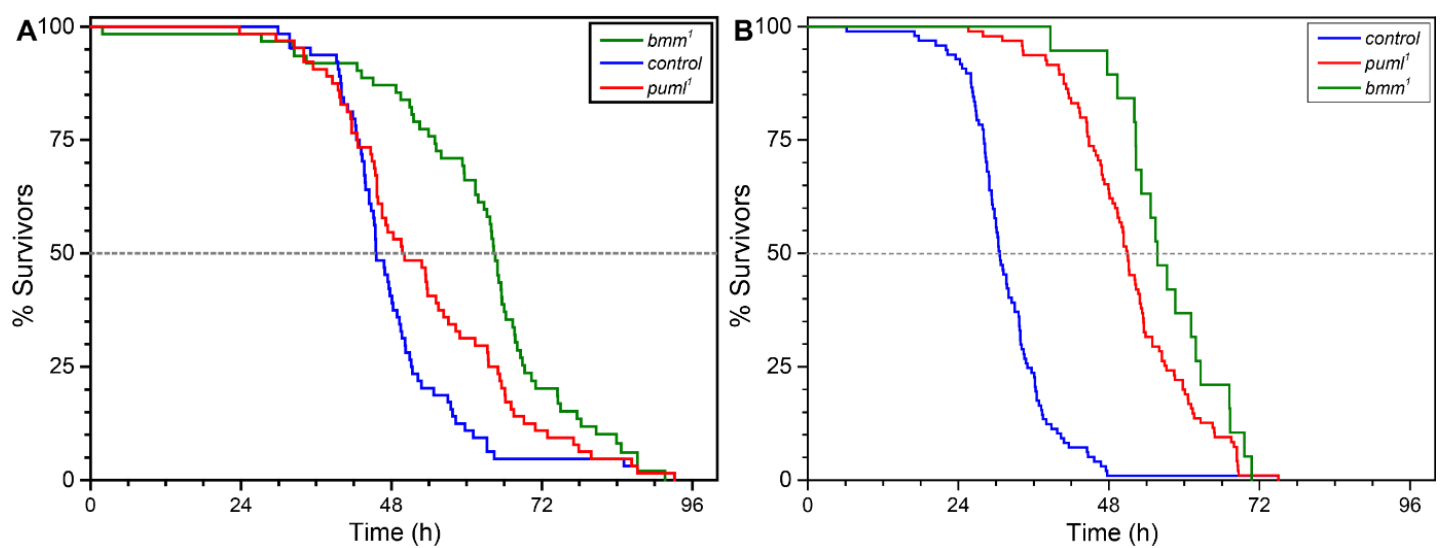

Figure 18 Mortality curve of $\mathrm{bmm}^{1}$, puml ${ }^{1}$ and control flies under food-deprivation. Starvation resistance of puml $\mathbf{l}^{1}$ comparable to $\mathbf{b m m}^{1}$ is significantly increased $(A, B)$ (log Rank- $P$ test; $n=64$ animals/genotype in $A, n=100$ for puml ${ }^{1}$ and control and $n=20$ for bmm $^{1}$ in $B ; P<0.001$ in both experiments). The two independent experiments show a strong variability of the used control flies in the median survival time under starvation. 


\subsection{Enzymatic characterization of Puml}

\subsubsection{Puml is not the activator of $\mathrm{Bmm}$ and has no triacylglyceride hydrolase activity}

The high similarity of Puml (Figure 10) to the mammalian paralogs ABHD4 and ABHD5 implied a possible conservation of the core lipid mobilization module formed by ATGL-ABHD5 (Lass et al., 2006, Yamaguchi et al., 2007) or a direct enzymatic activity like ABHD4 (Liu et al., 2008). puml and bmm are expressed at higher levels during starvation and the absence of each single one or both leads to increased body fat storage in form of TAGs. Additionally, pum/ ${ }^{1}$ and $\mathrm{bmm}^{1}$ flies are more starvation resistant (see above). In order to show that Puml directly stimulates Bmm's a triacylglyceride hydrolase activity both proteins were recombinantly expressed and lysates from overexpressing cells were used in a triacylglyceride hydrolysis assay. Protein expression was controlled by SDS-PAGE and subsequent Commassie staining. Expression of His-tagged proteins was checked by Western Blot analysis using an antibody against the His-tag (using a HRP-coupled secondary antibody). $\beta$ Galactosidase ( $\beta$-Gal.) was expressed as negative control. Whereas Bmm-GST (Figure 19) was expressed in E.coli the remaining proteins with an $\mathrm{N}$-terminal His-tag (Figure 19, B and C) were produced by COS-7 by cells (Schweiger et al., 2014).

Cell lysates (from overexpressing cells) were used for an in vitro lipid hydrolase assay using triolein (spiked with $[9,10(\mathrm{~N})-3 \mathrm{H}]$-Triolein) emulsified with a mixture of Phosphatidylcholine/Phosphatidylcholine (PC/PI). The radioactive TAG hydrolase assays were performed by Dr. Christoph Heier (Karl Franzenz University, Graz). ATGL and Bmm containing lysates exhibited a basal hydrolase activity in comparison to the control lysate containing $\beta$-Galactosidase. Neither Puml nor ABHD5 had a significant hydrolase activity on triolein. The co-incubation of ATGL with ABHD5 resulted in a stimulated hydrolase activity ( +4 fold). A comparable enhanced activity could not be observed for the incubation of Bmm with Puml. Inter-species combinations of Puml, ABHD5 with Bmm or ATGL could not reveal an evolutionary conservation of the activation property of ABHD5 on ATGL for its TAG hydrolase activity. This finding 
A

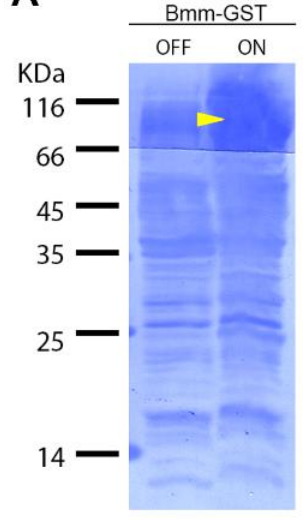

B

$\mathrm{KDa}$

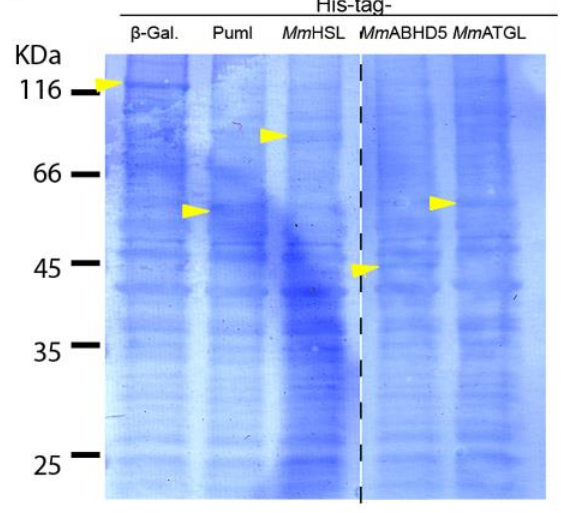

C

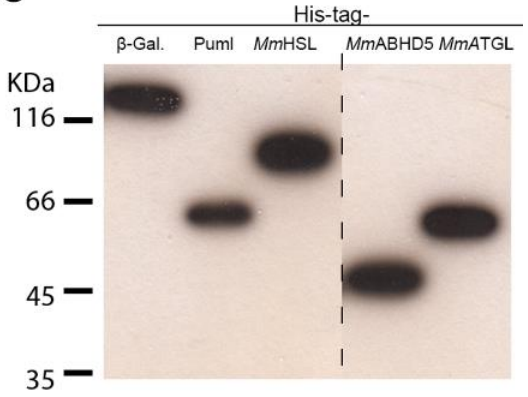

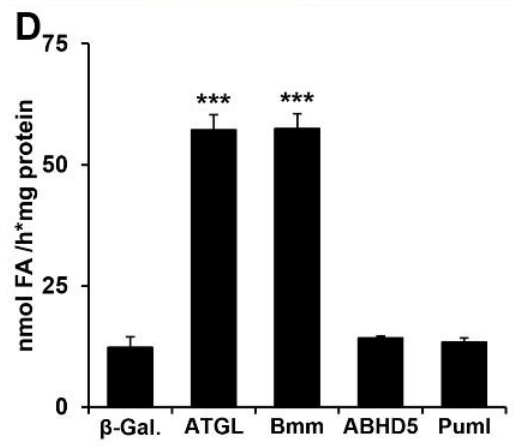

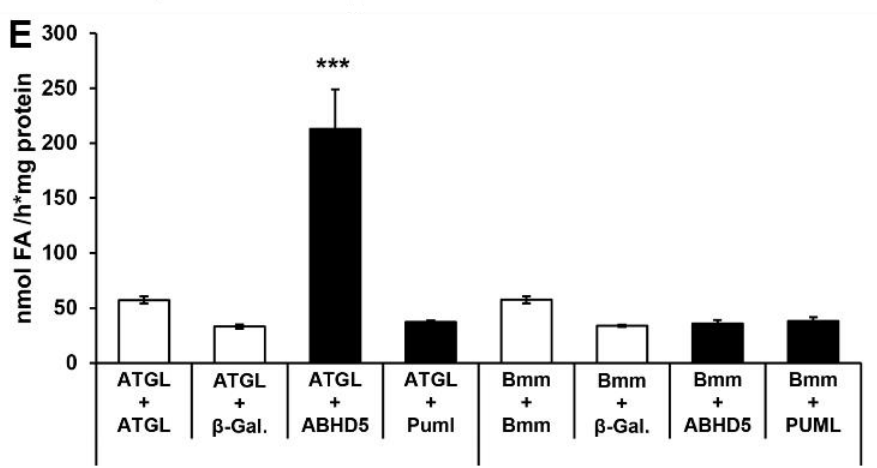

Figure 19 Puml cannot hydrolyse Triolein and does not stimulate Bmm lipase activity. Recombinant protein expression of Puml and Bmm for Triacylglyceride hydrolase assay. (A) Commassie stained SDSPAGE. Bmm-GST (83,2KDa) expression was clearly visible in induced cells (IPTG, $1 \mathrm{mM}$ added at $O D_{600}=0.5-0.6$, cultivation for $3 \mathrm{~h}$ at $22^{\circ} \mathrm{C}$ ). (B) Commassie stained gel of His-tagged proteins size separated by SDS-PAGE and (C) Western Blot from a parallel run gel (protein detection using an $\alpha$-His antibody). Expression could be detected for: $\beta$-Gal. (116KDa), Puml (50,4KDa), MmHSL ( 85KDa), ABHD5 $(39,1 K D a)$ and MmATGL $(55,3 K D a)$. (D, E) Calculated hydrolase activity of the lysates with the overexpressed proteins based on the amounts of released fatty acids in the Triacylglyceride hydrolase assay. Protein expression was performed by me (Plasmids used were obtained as mentioned in the Material and Methods part), radioactive triglyceride hydrolase activity assays were performed by Dr. C. Heier (Karl Franzenz University, Graz). Calculations were performed as described in chapter 2.11.1. ATGL and Bmm have basal TAG hydrolase activity (D). Co-incubation of ATGL and ABHD5 results in stimulated hydrolase activity compared to $A T G L+\beta$-Galactosidase. Bmm activity is not enhanced by coincubation with Puml or ABHD5 (E). Plotted are the means $\pm \operatorname{SEM}(D, E)$, Student's test, $* * *=P<0.001$ (Statistical tests were performed using following controls; (D) $\beta$-Galactosidase lysate; (E) ATGL or Bmm $+\beta$-Galactosidase mixtures).

corresponds with additional experimental data from Catharina Ebner and Dr. Christoph Heier (Karl Franzenz University, Graz, unpublished data). Therefore, Puml and Bmm seem not to directly interact in order to achieve a higher hydrolase activity on TAG.

\subsubsection{Recombinant Puml has phospholipase activity}

Different to ABHD5, that lost its catalytic center in its Serine-hydrolase motif by a point mutation (GxNxG), ABHD4 as well as Puml both have an active Serine-hydrolase motif. ABHD4 is directly involved in the regulation of brain $\mathrm{N}$-acyl phospholipids (Lee 
et al., 2015). A pilot experiment by Maria Pribasnig (Karl Franzenz University, Graz) indicated a possible activity of Puml, like for ABHD4, on the N-acyl phospholipid NAPE (1,2-dioleoyl-sn-glycero-3-phosphoethanolamine-N-arachidonoyl). In order to confirm this and to find possible hydrolysis targets for Puml, a substrate screen with neutral and polar lipids was performed. For this puml and as a negative control $\beta$ Galactosidase were over-expressed in insect cells (Hi-5 cells).
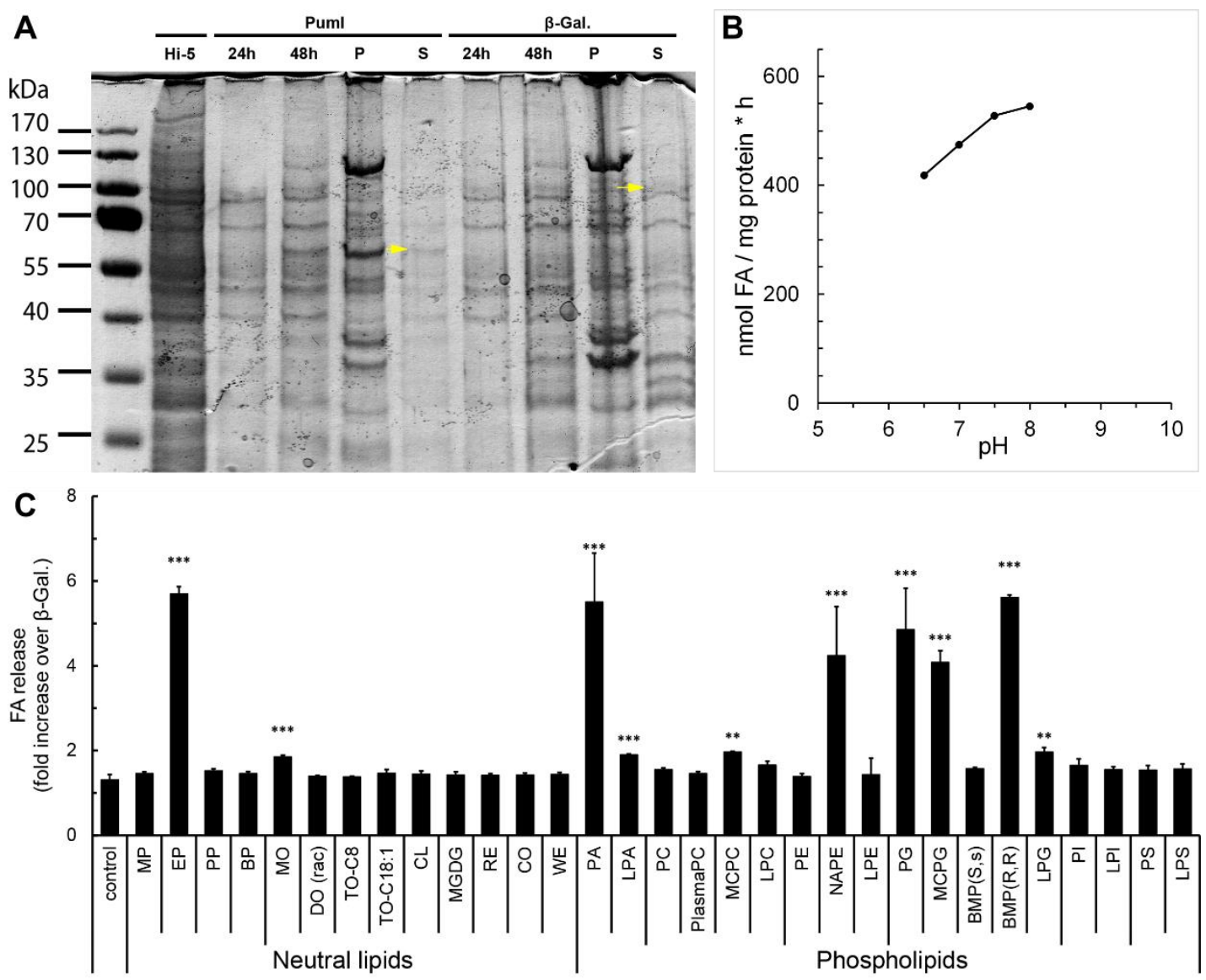

Figure 20 A Substrate screen identifies Puml as a potent phospholipase. (A) Puml and $\beta$-Galactosidase are expressed in insect cells (Hi-5). Expression of Puml (see arrow in A) increased with longer cultivation time with a peak during the drop of cell viability to under $70 \%$. The protein could be found in the $1000 \times \mathrm{g}$ pellet after cell lysis and in the supernatant used for the enzymatic assays (shown is a Commassie stained SDS-Page). (B) Hydrolase activity of Puml on NAPE, monitored by quantification of FA release from the substrate, showed an a preference for basic $\mathrm{pH}$. (C) Substrate screen for Puml. Data is represented as mean fold increase in FA release from the substrates in comparison to $\beta$ Galactosidase. Puml exhibits various substrate affinities: Phosphatidic acid (PA), Phosphatidylglycerol (PG), NAPE (N-acylphosphatidylethanolamine), medium chain Phosphatidylcholine (MCPC), mediumchain Phosphatidylglycerol (MCPG), Bis(Mono-acylglycerol)-phosphate (BMP[R,R]) and Ethyl palmitate (EP). Besides a low enzymatic activity on Mono-olein, Puml shows no affinity in hydrolysis neutral lipids (e.g. TAGs [TO-C8; TO-C18:1], DAGs [DO(rac)], Retinylester (RE). More detailed descriptions on the substrates can be found in the material and methods part. Plotted are means \pm SEM, Mann-Whitney test, ${ }^{*}=P<0.05, * *=P<0.01, * * *=P<0.001$. 
High FA release in comparison to control ( $\beta$-Galactosidase expressed) lysates could be observed for (Figure 20): Phosphatidic acid (PA), Phosphatidylglycerine (PG), NAPE (N-acylphosphatidylehtanolamine), MCPC, MCPC and $B M P(R, R)$. Besides Ethyl palmitate and a low affinity for Monoolein, neutral lipids were no preferred hydrolysis targets for Puml. Therefore, Puml does not contribute to the hydrolysis of storage fat. Puml showed a high activity in neutral to basic $\mathrm{pH}$. (Figure 20). Curvefitted data from substrate saturation measurements revealed a $\mathrm{K}_{M}$ of $\sim 0.78 \mathrm{mM}$ for Phosphatidic acid (PA) and $\sim 0.11 \mathrm{mM}$ for Phosphatidylglycerol (PG) showing a preference for the latter one. Interestingly, $\mathrm{v}_{\max }$ values for PA were $\sim 2.5$ fold higher than PG. This indicates that the TAG over-storage might be caused by a different mechanism than just a lower mobilization of storage lipids in pum $/^{1}$ flies.

\subsection{Energy storage of pummelig mutant flies under fed and stress conditions}

\subsubsection{Glycerolipid consumption under starvation is higher in pummelig mutants compared to control flies}

As shown before pum ${ }^{1}$ flies have an increased starvation resistance and store more TAGs than control flies. However, a direct hydrolytic activity on lipids (TAGs and DAGs) could not be detected. Perhaps, this is caused by a lower mobilization rate of storage lipid. In order to test of if lipid mobilization was impaired in pum/ ${ }^{1}$ flies a starvation assay was performed. Compared to $\mathrm{bmm}^{1}$, the general capability to mobilize storage lipids was not impaired in pum/ ${ }^{1}$ during starvation (Figure 21).

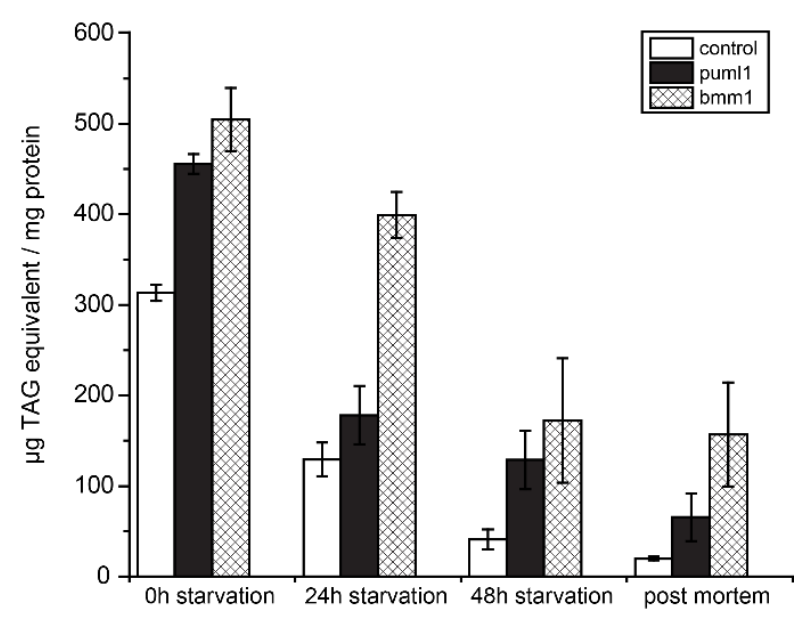

Figure 21 pum $^{1}$ flies mobilize lipids faster during starvation than control flies. Plotted are means of TAG \pm SEM. Both, puml ${ }^{1}$ and bmm $^{1}$ exhibit increased body fat storage at starvation start (six days after eclosure; one-way ANOVA $\left.F_{(2,8)}=19.03, P=9.09^{\wedge} 10^{-4}\right)$. During starvation, all tested flies could mobilize 
storage lipids (two-way ANOVA, genotype and starvation time as fixed effects, $F_{(2,31)}=72.41$, $\left.P=4.23^{\wedge} 10^{-14}\right) . b m m^{1}$ showed a slower TAG mobilization during the first $24 \mathrm{~h}$ of starvation and a substantial amount of TAGs remained immobilized in dead flies. puml $I^{1}$ mobilized around half of the storage lipids (much more then control flies) within the first $24 \mathrm{~h}$ of starvation. Lipid utilization became less in the following time of starvation and a significant amount of lipids remained in dead flies compared to control flies (one-way ANOVA, $F_{(2,14)}=5.40, P=0.01$; Fisher's $\left.L S D P<0.05\right)$. Control flies exhibited a similar pattern of reduced lipid mobilization with prolonging starvation but nearly used up all stored lipids.

Indeed, puml ${ }^{1}$ flies used up $\sim 30 \%$ more lipids during the first $24 \mathrm{~h}$ of starvation compared to control flies. Besides a significantly higher amount of un-mobilized lipids found in post-mortem flies, in total pum ${ }^{1}$ utilized on average $\sim 60 \%$ more lipids than control flies during starvation time. Thus, puml ${ }^{1}$ is not crucial for lipid utilization in general. Nevertheless, it is possible that a subset of lipids is not mobilized in pum/ ${ }^{1}$ flies.

A
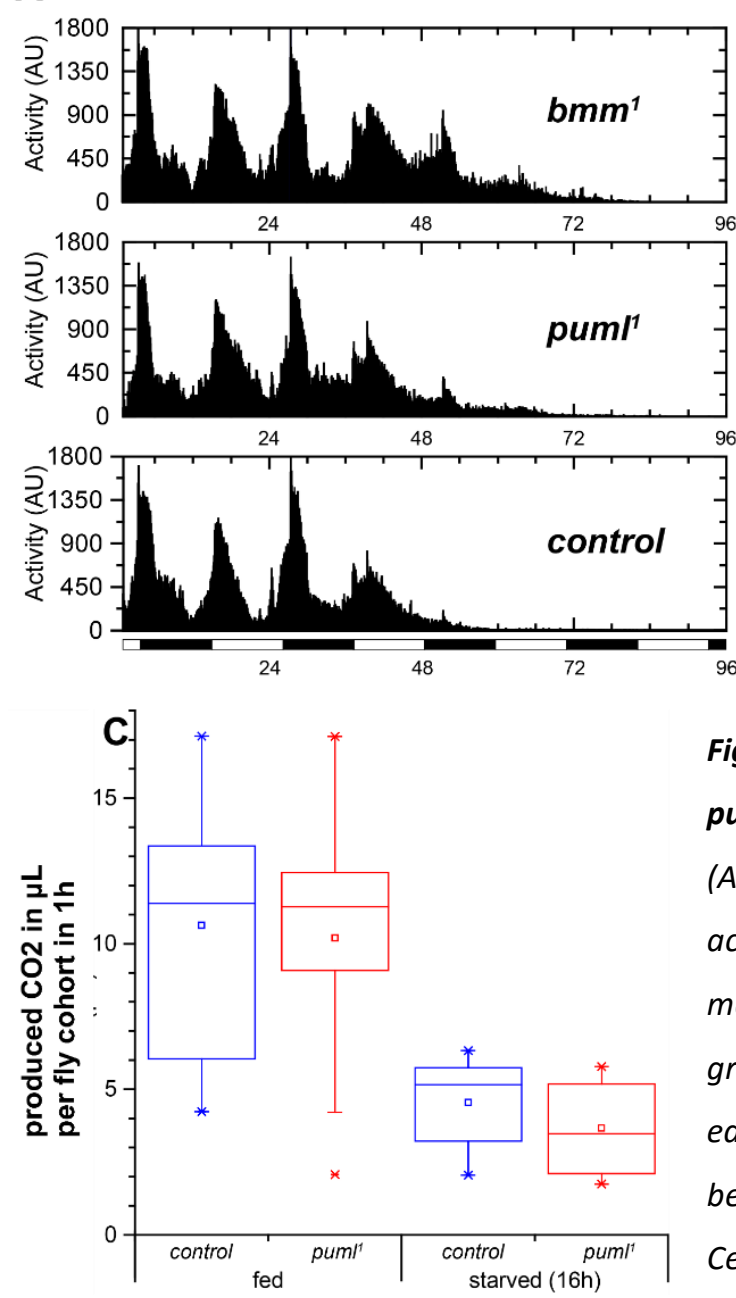

B

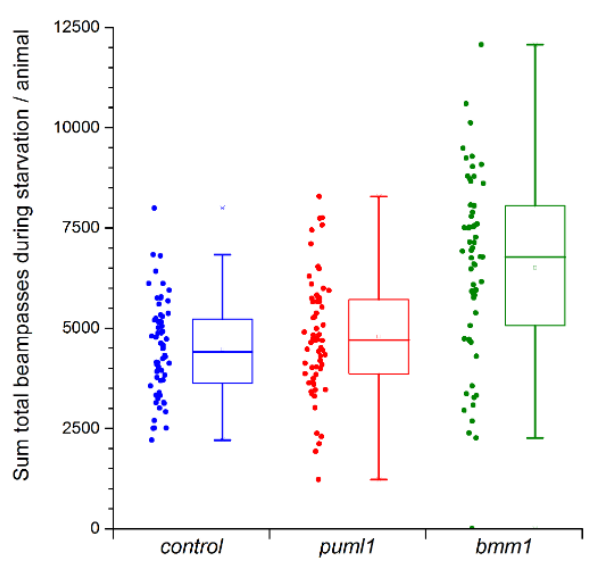

Figure 22 Locomotor activity and metabolic rate of pum ${ }^{1}$ are similar to control flies during starvation.

(A) For each fly line, the individual locomotor activity levels of individual flies ( $n=64)$ were measured in 5-minute bins. In order to generate a group profile, the sum of activity was plotted for each genotype. (B) Box plot for the total number of beam-passes during starvation time per animal; Center lines show the median, box limits indicate $25^{\text {th }}$ and $75^{\text {th }}$ percentiles as determined by OriginPro software; whiskers extend 1.5 times the interquartile range from the $25^{\text {th }}$ and $75^{\text {th }}$ percentiles; $n=63$ for each genotype. Average of total beampasses during starvation was significantly higher in $b m^{1}$ flies (Mann-Whitney test; ${ }^{* * *}=P<0.001$ ). (C) Metabolic rate is represented as box plot (generated as described shortly before). Metabolic rate was 
lower in starved (16 hours under starvation) cohorts (one cohort $=3$ animals; checked at zeitgeber $+8 h$ ) but no difference between pum ${ }^{1}$ and control flies could be detected (Mann-Whitney test).

\subsubsection{Total locomotor activity is not increased in pummelig mutant flies under starvation.}

In order to identify the cause for the increased lipid consumption in puml ${ }^{1}$ flies, locomotor activity form starving flies was monitored in the Drosophila activity monitoring system (Trikinetics). Single flies were kept in glass tubes and motion was quantified by counting passes through an infrared light beam. There was no difference in the activity between pum ${ }^{1}$ and control flies during starvation (Figure 22). Activity peaks were similar between all tested genotypes. Total starvation activity (Figure 22) was significantly higher in $\mathrm{bmm}^{1}$ compared to puml ${ }^{1}$ and control flies. Since median survival time under starvation (Figure 18) is increased in $b m m^{1}$ flies, allowing for a higher total activity, average total accumulated activity during starvation was increased in $\mathrm{bmm}^{1}$ flies. However, higher lipid utilization of pum/ ${ }^{1}$ flies, especially in the first twenty-four hours, was not correlated with higher activity during starvation.

\subsubsection{Metabolic rate is not changed in pummelig mutant flies}

Modulation of lipid storage in puml ${ }^{1}$ is different compared to control flies. During ad libitum feeding pum ${ }^{1}$ have increased body fat storage. Under fasting conditions these lipids are utilized faster than in control flies but locomotor activity is not changed. A possible reason for the higher lipid consumption might be a different regulation of metabolic rate in $p u m l^{1}$. In order to address this, a metabolic rate assay was performed using fed and fasted (16h under starvation) flies. Metabolic rate was significantly reduced under starvation compared to ad libitum fed flies (Figure 22). However, no difference in metabolic rate between puml ${ }^{1}$ and control flies was detected for fed as well as for fasted flies (Figure 22).

\subsubsection{Glycogen storage is decreased in pummelig mutant flies}

As shown before pum/ ${ }^{1}$ had higher lipid storage under fed conditions but faster utilization of this energy storage. pum ${ }^{1}$ flies did not have a higher activity under starvation and metabolic rates of were not different under feeding condition or during nutrient deprivation. As only the oxygen consumption was monitored for the 
metabolic activity and no data for $\mathrm{CO}_{2}$ was acquired, the oxidative quotient could not be used to identify which energy source is mainly used by the fly. Therefore, a more detailed time course experiment was performed to monitor energy stores during the first $24 \mathrm{~h}$ of starvation. Besides neutral lipids also protein content and glycogen stores were analysed (Figure 23). The amount of protein did not change significantly during the first $24 \mathrm{~h}$ of starvation, whereas lipids were utilized faster in pum/ ${ }^{1}$ flies. Interestingly, glycogen stores in pum ${ }^{1}$ were decreased by $\sim 42 \%$ compared to control flies (Student's test, $P=7,51 * 10^{-4}$ ) in fed flies. Glycogen stores depleted simultaneously with the lipids in pum ${ }^{1}$ and control flies (Figure 23). Due to the lower amount of glycogen in pum $/^{1}$ they were used up much faster leaving up lipids as the main energy source. Therefore, higher lipid mobilization of pum ${ }^{1}$ is mainly caused by lower availability of carbohydrates under nutrient deprivation.

A

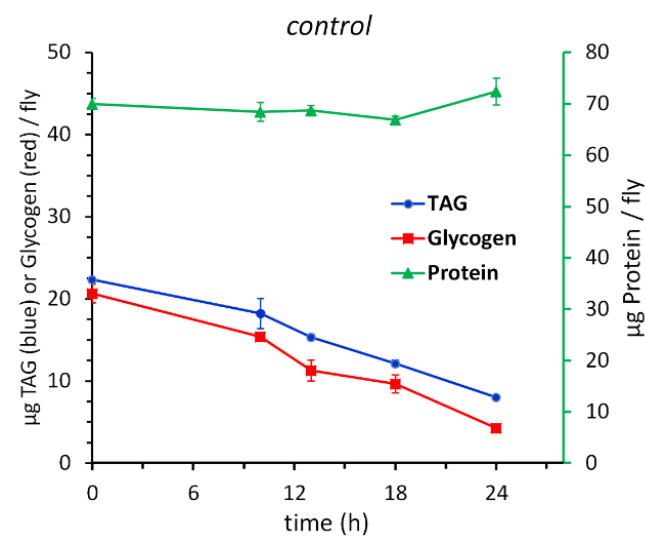

B

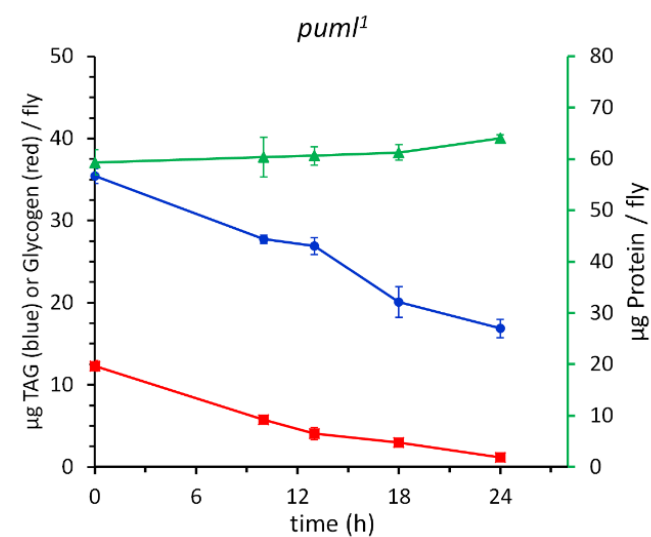

Figure 23 Less glycogen is stored in pum/ ${ }^{1}$ flies. Plotted are mean values for Protein (triangle; green), Triacylglycerides (dots; blue) and Glycogen (rectangle, red) \pm SEM during starvation. pum ${ }^{1}$ flies $(B)$ store increased amounts of TAG and have lower Glycogen stores (control flies (A) store $42 \%$ more Glycogen). Protein amounts were not changed significantly during the initial 24h of starvation. Glycogen stores in pum ${ }^{1}$ flies (B) were completely utilized during the observation time leaving lipids as the remaining major energy source.

\subsubsection{Desiccation resistance is impaired in pummelig mutant flies}

It is known that glycogen vastly contributes to desiccation resistance in Drosophila melanogaster (Marron et al., 2003). Therefore, it was tested if the reduced glycogen storage has a negative impact on the mean survival time under these conditions. In correlation with lower Glycogen storage in pum/ ${ }^{1}$ also mean survival time under desiccation was significantly decreased (-17\% see Figure 24$)$. 


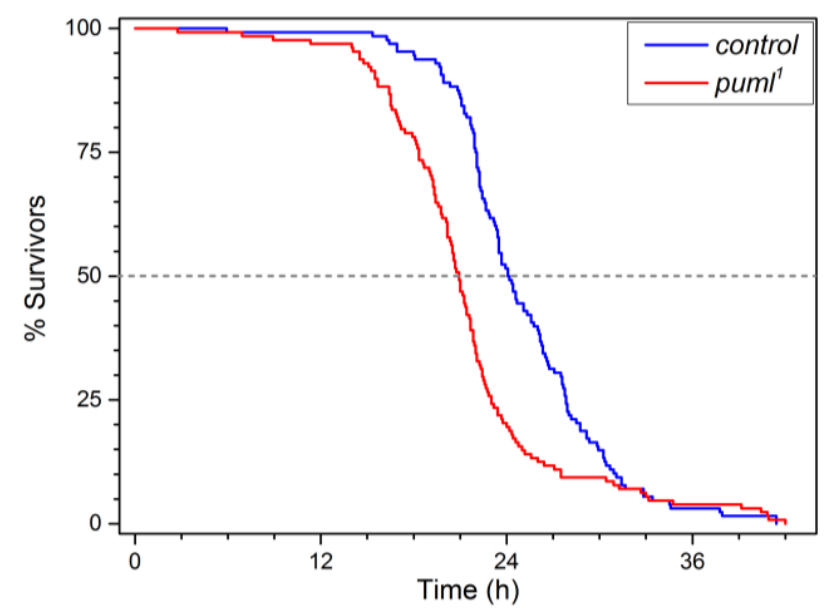

Figure 24 Desiccation resistance is decreased in puml ${ }^{1}$ flies ( $n=128$ animals per genotype; Log Rank test; $\left.P=5.41 * 10^{-6}\right)$.

Taken together, it was assumed that the TAG over-storage phenotype in pum/ ${ }^{1}$ flies was caused by an impaired mobilization of lipids. Interestingly, conversely to the high TAG storage, Glycogen stores in puml $^{1}$ flies were significantly lower in puml ${ }^{1}$ indicating that the storage type of energy in puml ${ }^{1}$ was changed. In general storage lipid mobilization was not impaired in puml $^{1}$ flies and metabolic rate was indifferent from control flies (under fed conditions and during starvation). However, puml ${ }^{1}$ utilized always more lipids during starvation than control flies indicating that lipids might be the preferred substrate for energy generation. The substrate screen identified Puml as an active phospholipase but how this effects lipid storage needs to be unravelled.

\section{6 pummelig mutant flies are normophagic}

As shown earlier lipid mobilization is actually higher pum/ ${ }^{1}$ flies but body fat storage is increased. Additionally, metabolic rate is similar to control flies. A possible increase in food intake and the resulting increased caloric loading could explain the increased body fat. Therefore, it was tested if pum $^{1}$ flies are hyperphagic. In order to address this question, individual food intake was measured using the CAFÉ system (as recently described (Baumbach et al., 2014a)). 


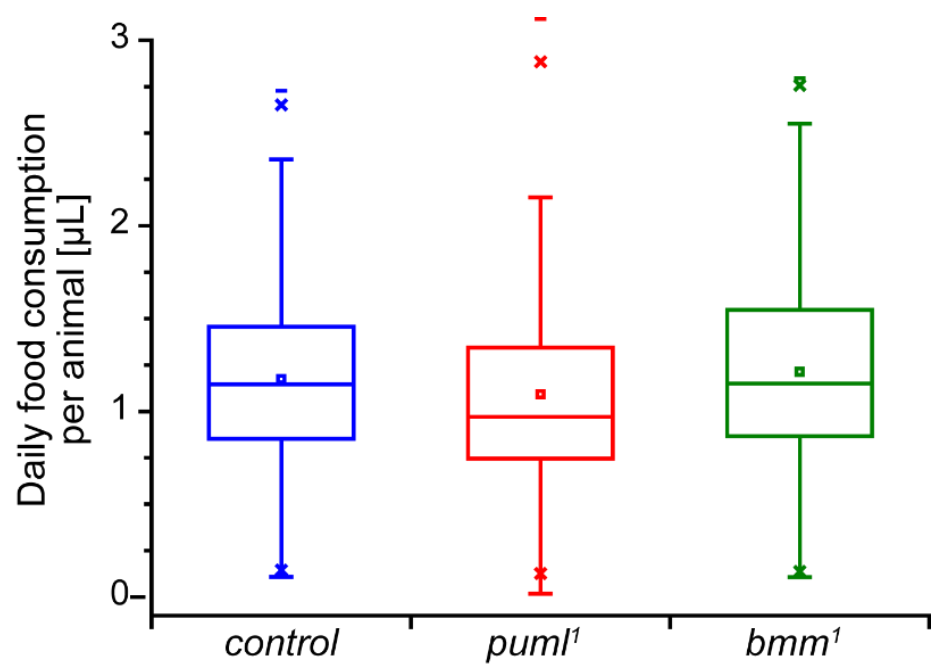

Figure 25 puml $^{1}$ and bmm $^{1}$ flies are not hyperphagic (one-way ANOVA, $F_{(2,407)}=1.92, P=0.14$ ). Box plot of the daily food consumption (5\% Yeast extract $+5 \%$ sucrose) of the tested genotypes (followed from six days after eclosure for six days). Center lines show the median, box limits indicate $25^{\text {th }}$ and $75^{\text {th }}$ percentiles as determined by OriginPro software; whiskers extend 1.5 times the interquartile range from the $25^{\text {th }}$ and $75^{\text {th }}$ percentiles; $n \geq 136$ for each genotype.

$b m m^{1}$ were checked additionally as these flies are also obese and to see if there is a comparable mechanism in these flies. Food intake varied from day to day but average food intake over six days was not significantly changed in the observed genotypes (Figure 25). However increased body fat storage of puml ${ }^{1}$ flies compared to control flies was persistent in the CAFÉ system. Thus, an altered feeding behaviour does not seem to be the cause for the observed changes in the TAG storage.

\subsection{Lipogenesis is increased in pummelig mutant flies}

With a comparable food intake and the knowledge that puml $I^{1}$ have lower glycogen storage I assumed that puml may acts as a metabolic modulator changing the preference for an energy storage towards in lipids (on the cost of carbohydrates) in pum ${ }^{1}$ flies through an unknown mechanism. In mice it could be shown that the absence of ABHD5 promoted the metabolic switch of cells towards aerobic glycolysis (Ou et al., 2014). In order to monitor if lipogenesis, pum/ ${ }^{1}$ flies were transferred on a diet with a radioactive tracer (uniformly ${ }^{14} \mathrm{C}$-labeled glucose) for $24 \mathrm{~h}$ (pulse flies) and radioactivity was measured in lipid extracts from total flies (in principle as described in (Katewa et al., 2012)). 

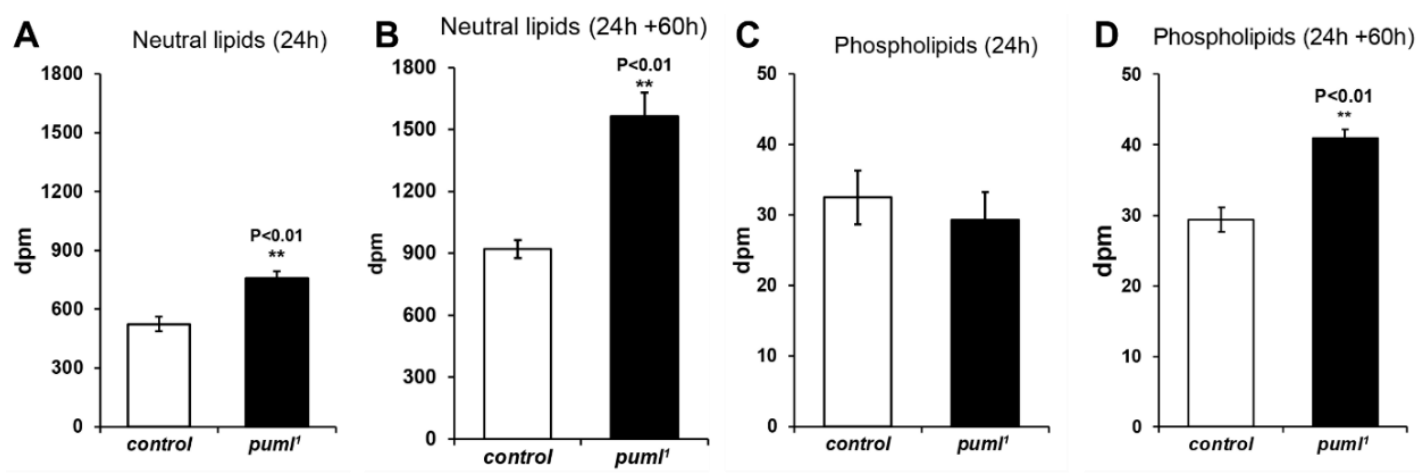

Figure 26 Rate of de novo lipid synthesis is increased in puml ${ }^{1}$ flies. Plotted are means of dpm (disintegrations per minute) \pm SEM (Student's test; $n=5$ ) measured in neutral lipid $(A, B)$ and phospholipid fractions $(C, D)$ from 20 flies after $24 \mathrm{~h}$ on diet supplemented with ${ }^{14} \mathrm{C}$ glucose and a second measurement after additional $60 \mathrm{~h}$ on non ${ }^{14} \mathrm{C}$ glucose diet. Rate of de novo lipid synthesis is significantly increased in puml ${ }^{1}$ generating more lipids with ${ }^{14} \mathrm{C}$ incorporated lipids within $24 \mathrm{~h}(A)$ on this diet. The difference in amounts of labelled neutral lipids is maintained during the chase phase indicating no utilization of the generated lipids (B). Amounts of synthesized phospholipids were similar after $24 \mathrm{~h}$ feeding on ${ }^{14} \mathrm{C}$ diet $(C)$. Significant differences could be detected after continuous time on non-labelled diet (D).

Lipids were fractioned into neutral and phospholipids and analysed separately. Both genotypes exhibited de novo lipid synthesis (measured by decay of incorporated ${ }^{14} \mathrm{C}$ into lipids from the fed ${ }^{14} \mathrm{C}$ glucose) under the experimental conditions and food regime. Measured radioactivity in the neutral lipids fraction was significantly higher in pum ${ }^{1}$ compared to control flies. The amounts of labelled phospholipids were similar between the tested genotypes. Therefore, pum/ ${ }^{1}$ flies appear to generate more storage lipids although food intake is not increased. Even though flies were kept on regular diet for additional $60 \mathrm{~h}$ (without ${ }^{14} \mathrm{C}$ glucose; Chase flies), amounts of labelled neutral lipids were increased in both genotypes compared to flies collected directly after the $24 \mathrm{~h}$ pulse. This indicates that de novo lipid synthesis from ingested ${ }^{14} \mathrm{C}$ glucose continued after the feeding phase that might be driven by stored ${ }^{14} \mathrm{C}$ glucose supplemented food in the crop. Utilization/turnover of the labelled lipids cannot be addressed by the used experimental approach. It is possible that utilization of lipids is not occurring and not clear if the maximal incorporation of ${ }^{14} \mathrm{C}$ glucose is or has been reached in the tested +60 h samples. At least phospholipid levels in control flies were stable in at both time points arguing in favour of higher lipogenesis rates in puml ${ }^{1}$.

Taken together puml ${ }^{1}$ lipid over-storage phenotype in adult flies is rather the result of a preference to store energy as neutral lipids than carbohydrates. Reduced 
glycogen storage, unchanged metabolic rate in $\mathrm{puml}^{1}$ and increased lipogenesis support this model. Future experiments should also address the incorporation of ${ }^{14} \mathrm{C}$ glucose into glycogen in order to get a better insight into the channelling of the fed glucose into the different energy stores.

\subsection{Localization of Puml and lipid storage phenotypes in pummelig mutant flies}

\subsubsection{Puml is a member of the lipid droplet proteome}

Various studies identified Puml as a lipid droplet (LD) resident. It was found on embryonic LDs (Cermelli et al., 2006). Previous work (in our lab) on Puml by Anna Takács using a C-terminal labelled Puml::mCherry fusion protein (expressed by using the target system and expression under endogenous promotor) showed a LD association in larval fat body and free floating adipocytes (Takacs, 2007). Jonathan Rosenberg (a former bachelor student in the lab) located the same C-terminal tagged protein constructs on LDs in larval wing discs, the proventriculus and on LDs in Malpighian tubules in larvae and adult flies (Rosenberg, 2012). More recently, a different C-terminal labelled (mCherry) construct was expressed in the Drosophila melanogaster S2-cell line that localized on LDs in these cells (Krahmer et al., 2013) as well.

First, the localization of Puml::mCherry on LDs was verified in the adult fat body.

Localization of Puml::mCherry was comparable to the published lipid droplet resident Brummer (Grönke et al., 2005) that showed a uniform and dot-like distribution on LDs in the adult fat body. Puml::mCherry fusion protein in accordance with previous findings for Puml localization (described above) could be found on LDs in adult fat body cells as well. Most of the Puml::mCherry exhibited a uniform/ring-like distribution on LDs but a portion showed accumulations in close proximity to LDs or distant from lipid-dye stained structures. 

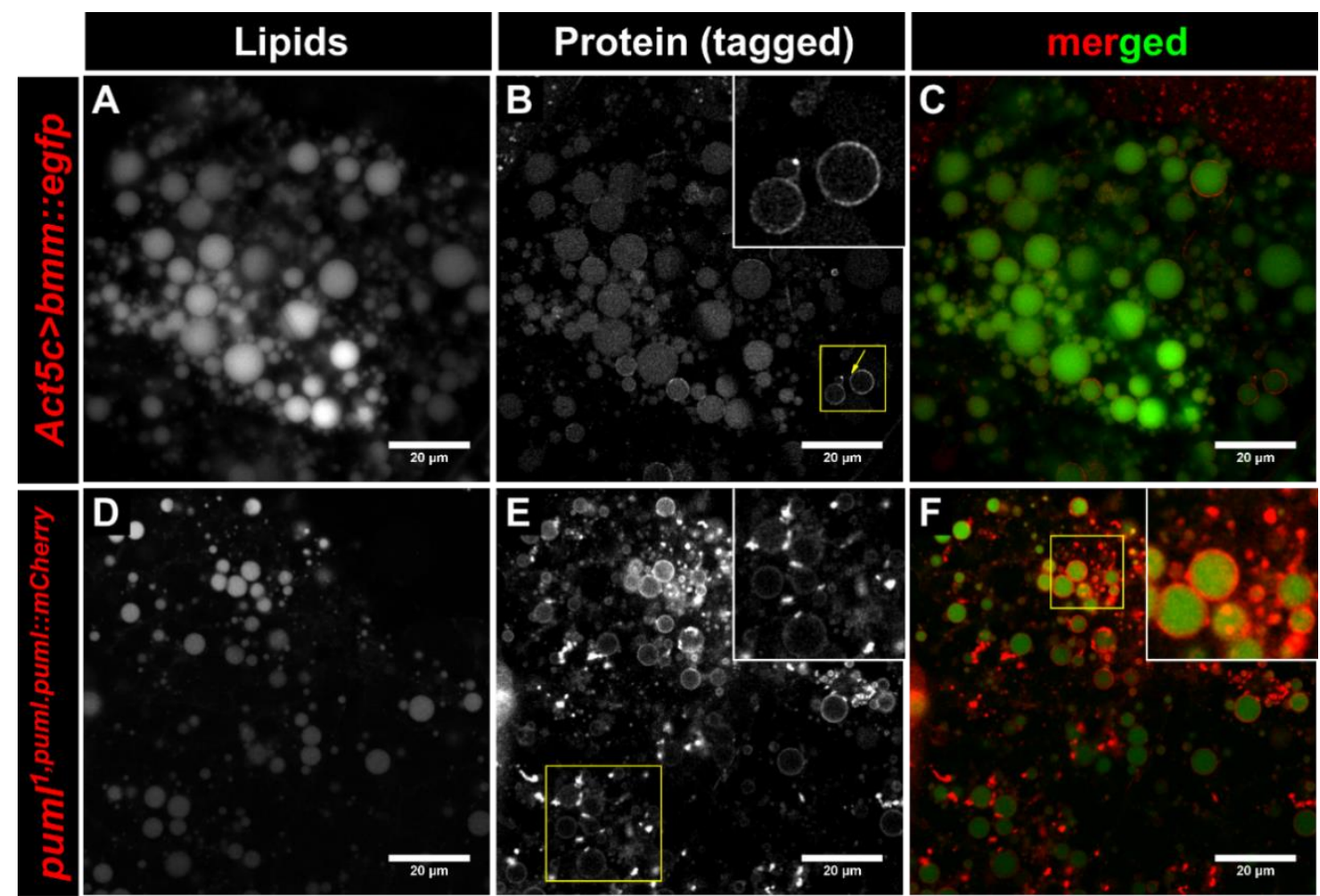

Figure 27 Puml::mCherry and Bmm::EGFP are localized to lipid droplets in adult fat body tissue (C, F). Bmm::EGFP (Lipids stained with LIPID TOX) exhibits homogeneous and dot-like localization (A-C; Box in B). Puml::mCherry localized on also on lipid droplets and showed a uniform/ring-like localization ( $D$ F) as well as local accumulations on the LDs surface or in very close proximity to it (E in the Box, arrow). Additionally, accumulations not overlapping with neutral lipid staining (Bodipy493/503) could be seen for Puml::mCherry (see Box in F).

\subsection{2 pummelig mutant flies exhibit ectopic lipid storage in Malpighian tubules}

In order to find out where Puml::mCherry accumulates besides lipid droplets I switched to a more accessible cell biology system in the fly. Prior work on puml function concentrated on finding a biological phenotype in the pum/ ${ }^{1}$. Whereas increased total body fat storage was shown in this work for the first time a screening for possible lipid accumulation phenotypes in pum/ ${ }^{1}$ was performed by Jonathan Rosenberg. Based on the expression data for pummelig (CG1882) obtained from the FlyAtlas project (Chintapalli et al., 2013) various tissues were screened. The strongest change in lipid storage (followed by Oil Red O staining) of pum/ ${ }^{1}$ correlated with the highest expression of pum/ in Malpighian tubules (Rosenberg, 2012), the renal organs of the fly. A comparable over-storage of lipids could also be observed in $\mathrm{bmm}^{1}$ (Rosenberg, 2012) using Oil Red O to stain for lipids.

During this work the over-storage phenotype in Malpighian tubules could be confirmed for both puml ${ }^{1}$ and $b m m^{1}$ (Figure 28) using Oil Red O staining, fluorescent 
lipophilic dyes (e.g. Bodipy493/503) and electron microscopy (sample preparation and image acquisition were performed by Dr. Dietmar Riedel (MPI-bpc)). Compared to pum/ that has a very high expression in Malpighian tubules, $b m m$ expression is only moderately in this tissue and compared too other $\mathrm{bmm}$ expressing tissues quite low. Nevertheless, increased lipid storage in Malpighian tubules of $b m m^{1}$ flies exceeds the levels of puml ${ }^{1}$ flies. An electron microscopic analysis could confirm the particles as lipid droplets and no obvious changes (besides more lipid droplets) in cellular morphology of Malpighian tubules could be observed in both mutants (pum/ ${ }^{1}$ and $\left.b m m^{1}\right)$.

\subsubsection{1 pummelig expression in pummelig mutant flies can rescues the lipid over- storage phenotype}

As shown before in this work, an overexpression of puml had no effect on body fat storage in the fly. In order to see if it is functional and capable of reversing/prohibiting the lipid over-storage phenotype it was expressed in puml ${ }^{1}$. A ubiquitous (Act5c>GAL4) and tissue specific expression in the fat body (FB-SNS>GAL4) and Malpighian tubules (UO>GAL4) in pum/ ${ }^{1}$ flies resulted in a significant reduction in body fat storage in adult flies (Figure 29).

The ubiquitous expression of the puml resulted in a reduced body fat and reduced nearly absent lipid droplets in Malpighian tubules (Figure 29). When expressing the construct in Malpighian tubules specifically the effect on the body fat storage was much smaller but still significant and lipid over-storage phenotype of puml ${ }^{1}$ in the Malpighian tubules was reverted. A fat body specific expression on the other side had a strong effect on body fat but lipid over-storage phenotype in Malpighian tubules was not affected. Therefore, the used UAS-puml construct is functional and capable of reverting the lipid-over-storage. 

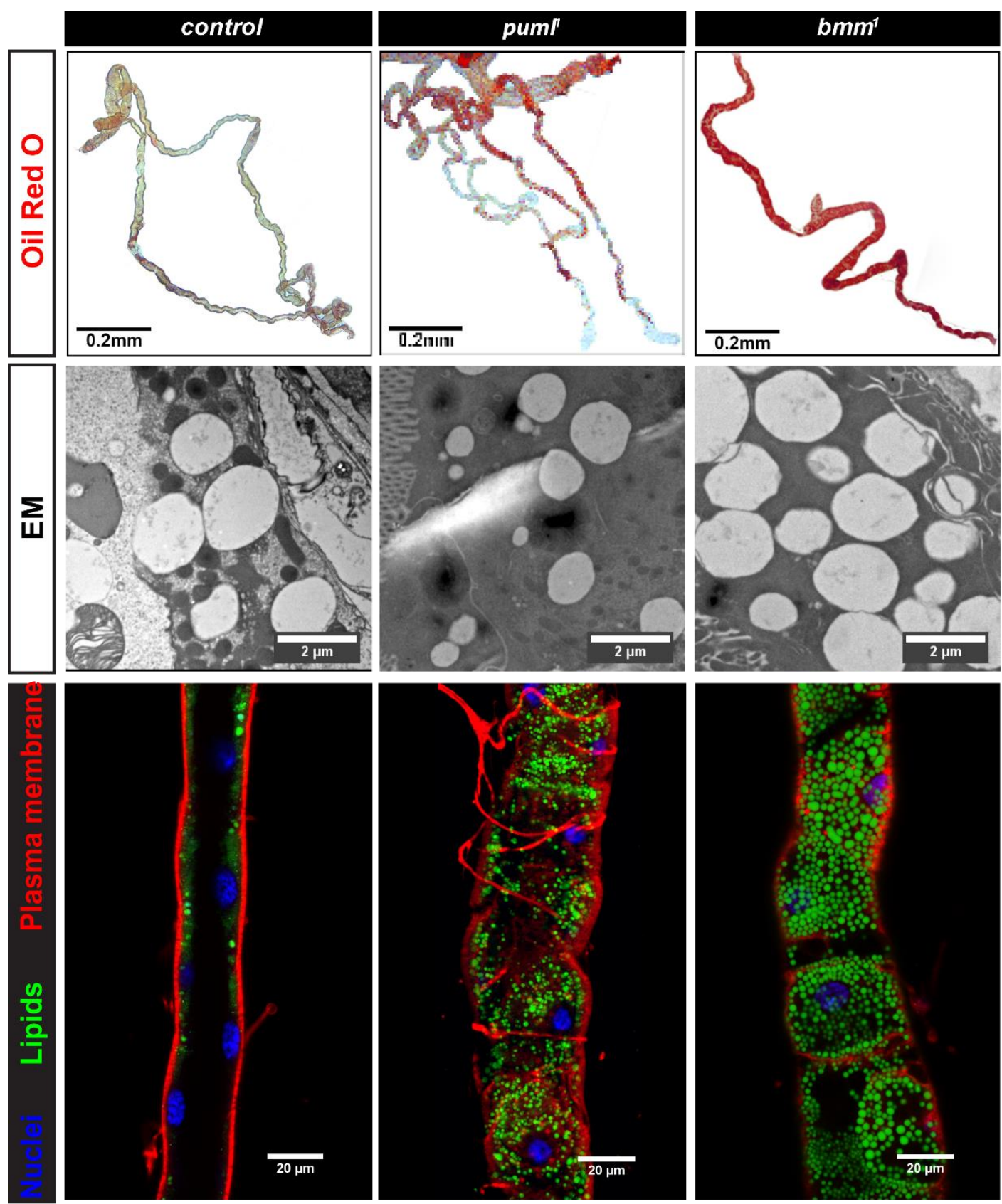

Figure 28 Lipid storage is drastically increased in Malpighian tubules of puml ${ }^{\mathbf{1}}$ and bmm $^{\mathbf{1}}$ flies. Upper Row shows an Oil Red $O$ staining in fixed Malpighian tubules from six day old adult flies. Mid row displays electron microscope pictures (high pressure freezing; sample preparation and image acquisition was performed by Dr. Dietmar Riedel; MPI-bpc). Fluorescence microscope pictures using DAPI for nucleic staining, Bodipy493/503 for lipids and Cellmask ${ }^{\text {TM }}$ for plasma membrane staining are shown in the lower row.

Lipid storage in general appears to be a native property of Malpighian tubules (see

Figure 28) and puml and bmm are involved in the modulation of this energy source.

Also the fact, that a fat body specific expression of pum in puml ${ }^{1}$ flies does not change ectopic lipid storage in Malpighian tubules drastically indicates that lipid storage in Malpighian tubules is not directly coupled to lipid storage in the fat body. 

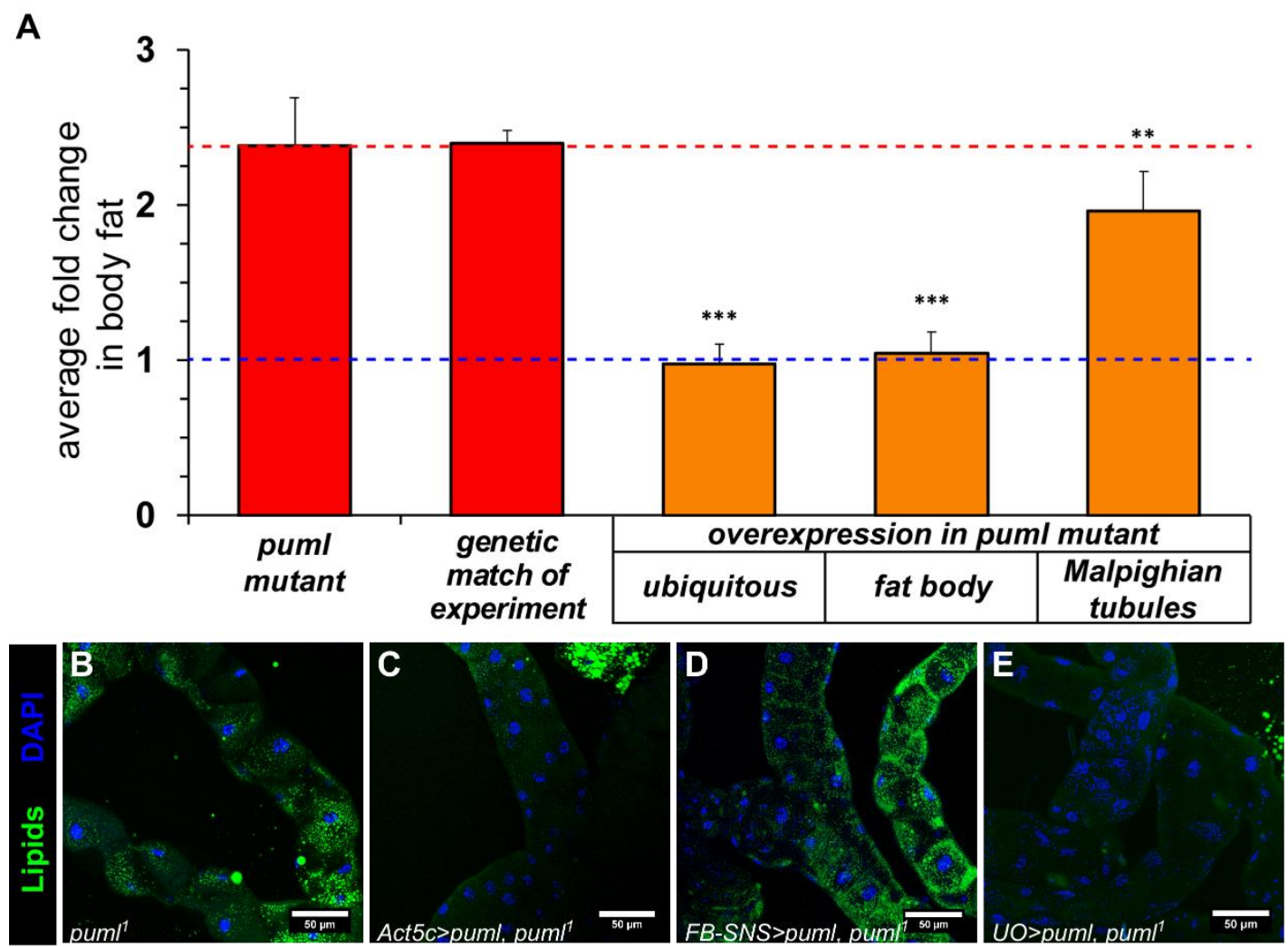

Figure 29 Overexpression of Puml rescues lipid over-storage phenotype in Malpighian tubules from pum $^{1}$ flies. Plotted are average fold changes of body fat ( \pm SEM) of pum ${ }^{1}$ flies and under the ubiquitous (Act5c>GAL4) or tissue-specific expression (FB-SNS>GAL4 [fat body] and UO>GAL4 [Malpighian tubules]) of pum/ compared to control flies and the genetic match of the experiment. Body fat is significantly lower during the expression of pum $/$ in puml ${ }^{1}$ flies. A ubiquitous (C) and Malpighian tubule (E) specific expression both reduce lipid storage in Malpighian tubules. A fat body specific expression leads to decreased body fat storage (A) but does not affect lipid stores in Malpighian tubules (D). Pictured are representative images from Malpighian tubules (mid-segment) stained with DAPI (for nucleic acids) and Bodipy(493/503) for lipids. (Student's test; ${ }^{* *}=\mathrm{P}<0.01, * * *=\mathrm{P}<0.001$ ). The dashed red line represents the fold change in body fat of pum ${ }^{1}$ flies compared to control flies (blue dashed line; represents body fat storage of genetically matched control to puml ${ }^{1}$ flies).

\subsubsection{Pummelig::mCherry fusion protein is localized on peroxisomes}

As shown before Malpighian tubules can store lipids and $\mathrm{bmm}^{1}$ and $p u m l^{1}$ flies exhibit elevated lipid storage in this tissue. An overexpression of pum/ in Malpighian tubules can reverse this phenotype. Therefore, I chose this biological cell system to investigate the localization of Puml::mCherry further as it was not only found on lipid droplets but also showed additional accumulations in the cytoplasm.

For Arabidopsis thaliana ABHD4/5 (At4g24160) it is known that it can localize to LDs (James et al., 2010) and interacts with PXA1 and can be found on or in association with Peroxisomes (Park et al., 2013, Park et al., 2014). Therefore, I checked if nonlipid associated Puml::mCherry signal could be a localization on Peroxisomes. Indeed, 
a co-localization could be observed with endogenously expressed Puml::mCherry in flies (Figure 30) with a ubiquitously expressed peroxisome targeted EYFP (Act5c> eYFP-Pts1; UAS-eYFP::Pts1 was a kind gift from Dr. J. Faust (Faust et al., 2014).

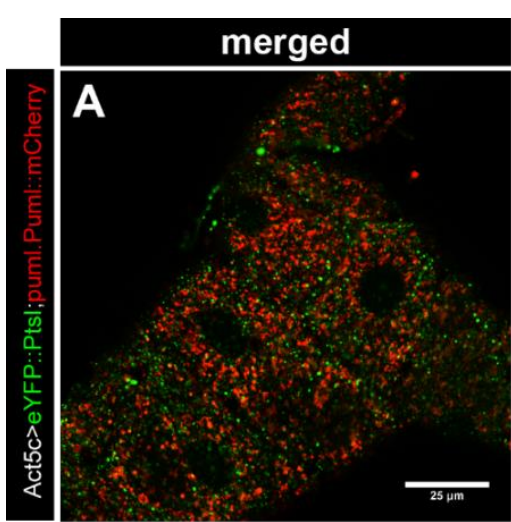

D

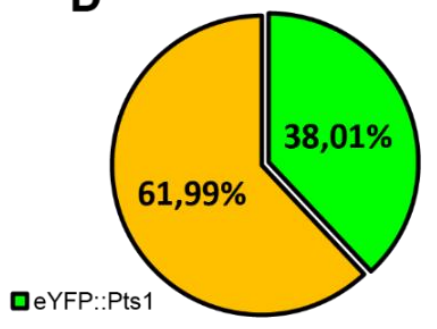

口eYFP::PtsI + Puml::mCherry

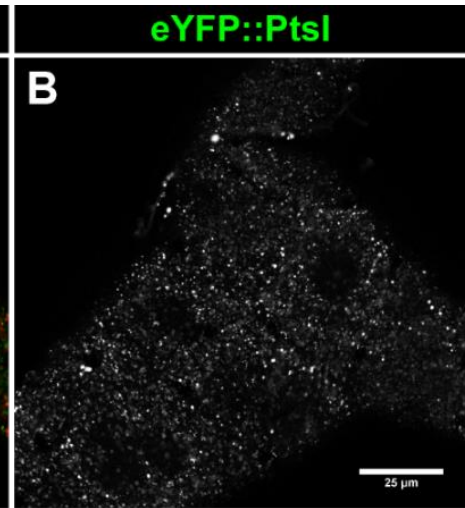

$\mathbf{E}$

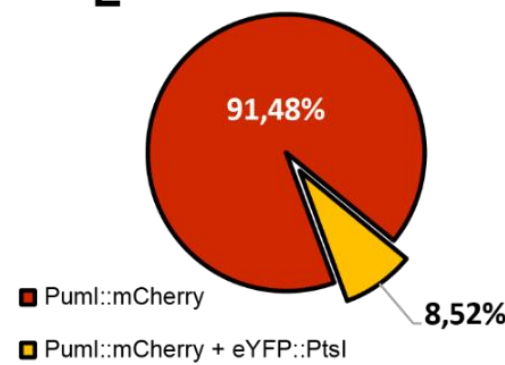

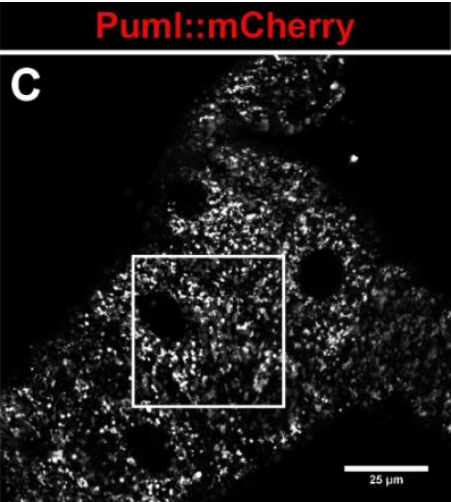

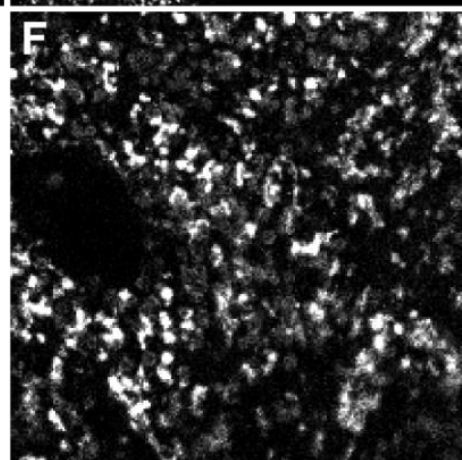

Figure 30 Puml::mCherry is localized on lipid droplets and peroxisomes. Puml::mCherry (expressed by the endogenous promotor) and eYFP::Pts1 (ubiquitously expressed: Act5c>GAL4) localization was analysed in puml ${ }^{1}$ genomic background. $~ 62 \%$ of eYFP::Ptsl signal overlapped (A) with Puml::mCherry (C) indicating a peroxisomal (B) abundance of Puml::mCherry. Less than $10 \%$ of the total Puml::mCherry signal exhibited a co-localization with eYFP::Ptsl. The majority of Puml::mCherry showed a ring-like distribution (potentially lipid droplets) and accumulations that neither were ring-like or co-localized with eYFP::PtsI (F). Image A, B, C and F show ureter of an adult fly.

The majority ( 92\%) of Puml::mCherry signal exhibits a circular pattern (potentially around lipid droplets) or roundish accumulations. The remaining $\sim 8 \%$ of the Puml::mCherry signal overlaps with $~ 62 \%$ of eYFP::Ptsl signal. With eYFP::Ptsl representing the peroxisomes it can be claimed that over half of the peroxisomes is populated with Puml::mCherry but the majority of the protein is not on peroxisomes. Interestingly, a peroxisomal targeting signal (PtsI) that consists of a C-terminal SKLmotif (Faust et al., 2012) is not conserved in Puml though this localization may requires an interaction partner. 


\subsection{Lipid over-storage in Malpighian tubules of pummelig mutant flies does not impair osmotic resistance}

Malpighian tubules perform various tasks in Drosophila melanogaster e.g. osmoregulation (Berridge and Oschman, 1969, Wessing and Eichelberg, 1969) and are important for immune response (McGettigan et al., 2005). More recently, it was shown that $\mathrm{bmm}$ and puml are upregulated under osmotic stress (Stergiopoulos et al., 2009). As lipid storage was altered in puml ${ }^{1}$ Malpighian tubules it was assumed that biological functions of this tissue might be impaired. Therefore, pum/ ${ }^{1}$ flies were subjected to diet containing high amounts of sodium chloride (Figure 31)

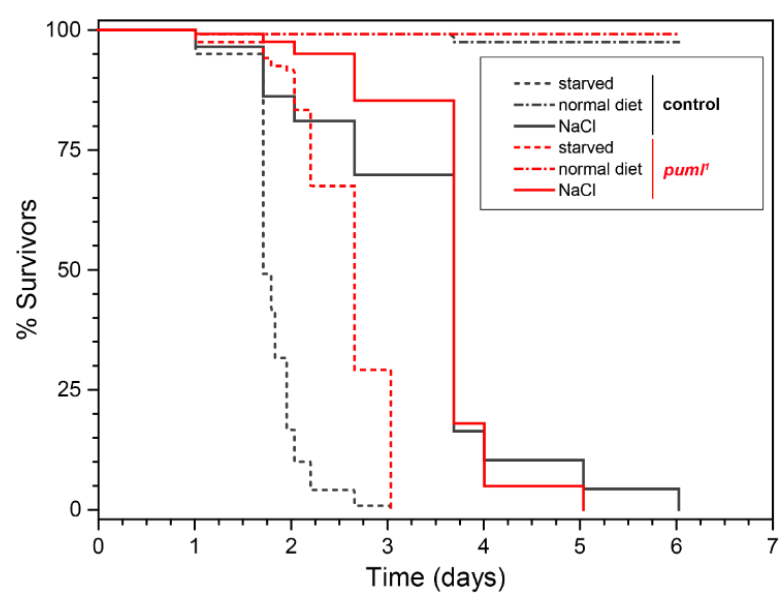

Figure 31 Osmotic stress resistance of pum ${ }^{1}$ flies is not impaired. Six-day old flies were transferred to either one of the following dietary regimes: normal food, starvation or food with $4 \% \mathrm{NaCl}$ added ( $n=120$ flies per tested diet). Flies survived longer on salted food compared to starved flies (log Rank test, $P<0.001$ ) and starved puml ${ }^{1}$ flies showed higher mean survival time than control flies (log Rank test, $P<0.001)$. Under osmotic stress (salty diet) no significant difference in the mean survival time could be observed between the two tested genotypes.

Compared to flies under starvation mean survival time on high salt diet was significantly increased. Although there is a significant difference in mean survival time between puml ${ }^{1}$ and control flies this difference cannot be observed under osmotic stress (Fig. X). So the increased lipid storage in Malpighian tubules of pum/ ${ }^{1}$ has no drastic negative effect on the osmoregulatory capacity of flies lacking puml.

\subsubsection{Lipid droplet distribution is altered in pummelig mutant flies}

As introduced before, body fat is increased in pum/ ${ }^{1}$ and lipid storage is elevated in Malpighian tubules that can be rescued by tissue-specific expression of puml-cDNA. Microscopic analysis of lipid storage using fluorescent lipophilic dyes indicated a difference in the lipid droplet size distribution between puml ${ }^{1}, \mathrm{bmm}^{1}$ and control 
flies. Due to the limited biological dimensions of Malpighian tubules, this tissue was used to analyse the lipid droplet size between these genotypes (Figure 32).

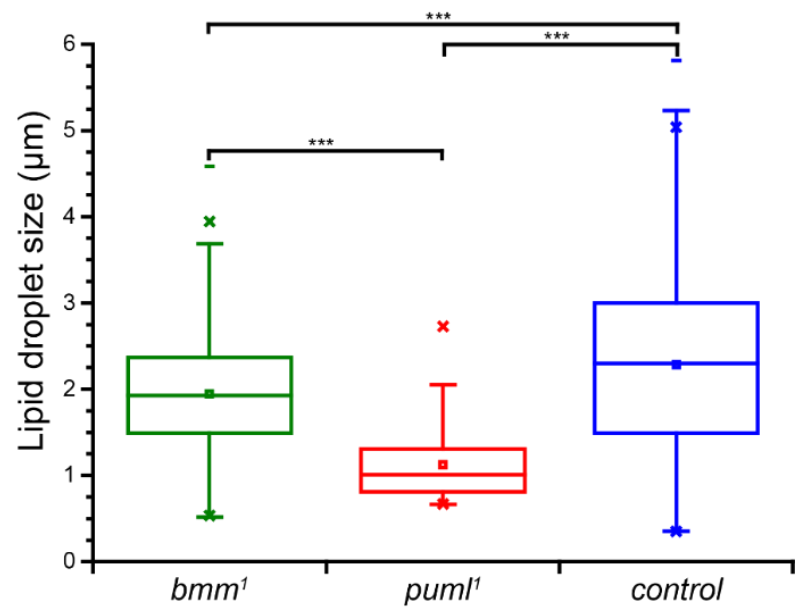

Figure 32 Average lipid droplet size (diameter) is reduced in Malpighian tubules of puml ${ }^{1}$ flies. Box plot of lipid droplet size quantified from confocal pictures of fluorescently stained lipid droplets in Malpighian tubules. Center lines show the median, box limits indicate $25^{\text {th }}$ and $75^{\text {th }}$ percentiles as determined by OriginPro software; whiskers extend 1.5 times the interquartile range from the $25^{\text {th }}$ and $75^{\text {th }}$ percentiles (Mann-Whitney test; $n_{\text {lipid droplets }}$ analysed per genotype: $\mathrm{bmm}^{1}=1206$, puml ${ }^{1}=1715$ and control=306).

Lipid storage is quite low in Malpighian tubules of control flies (Figure 32) reflected by the low number of individual lipid droplets that were used for the size distribution (diameter of lipid droplets was calculated from the total area from a maximum intensity projection of an acquired 3D-confocal image stack). Though, the quantified lipid droplets on average had the largest diameter compared to $\mathrm{bmm}^{1}$ and puml${ }^{1}$. As described earlier both mutants had a much higher lipid storage in Malpighian tubules but average lipid droplet (LD) sizes were significantly smaller. A possible explanation might be an alternated phospholipid metabolism in puml ${ }^{1}$ and $\mathrm{bmm}^{1}$ flies that finally effects also the phospholipid composition of the LDs and therefore shaping their size.

\subsubsection{Long-chain fatty acids and poly-unsaturated fatty acids are elevated in pummelig mutant flies}

Lipid stores are elevated in pum/1. The major neutral lipid class contributing to this are TAGs (Figure 14). During starvation lipids are mobilized in general but on average a significantly higher amount of lipids remain in dead animals (Figure 21). Additionally, lipid droplet size distribution in Malpighian tubules is changed in puml ${ }^{1}$. 
AtABHD4/5 null mutants (plants) exhibit similar to puml ${ }^{1}$ ectopic lipid storage in leaves (James et al., 2010). The major elevated neutral lipid class were TAGs and content of poly-unsaturated fatty acids (PUFAs) esterified in the neutral lipids were elevated (James et al., 2010) and a shift towards longer fatty acid sidechains of neutral lipids could be seen. Additionally, it had been shown that AtABHD4/5 interacts with the peroxisomal ABC-transporter 1 (PXA1) (Park et al., 2013). A single PXA1 mutant and a AtABHD4/5, PXA1 double mutant exhibited the same phenotype on TAGs as the single $A t A B H D 4 / 5$ mutant.

As shown before Puml is localized on lipid droplets and Puml::mCherry can be found on peroxisomes as well. Also, a portion of neutral lipids remains immobilized during starvation to death in puml ${ }^{1}$. Therefore, I hypothesized a comparable phenotype of changed TAG composition. In order to identify changes, the lipidome was analysed using mass-spectrometry. Malpighian tubules were explanted from adult pum/ ${ }^{1}$ and control flies on standard diet (density controlled). Lipid extraction and mass spectrometry were performed by Vinzenz Hofferek (pilot experiment; 5 independent extractions from each 10 MT pairs; MPIMP-golm; average standard deviation of Mol \% TAG species $=0,15 \%$ for both genotypes) and independently by Dr. Thomas Eichmann (University of Graz; 3 independent extractions from each 100 MT pairs; average standard deviation of Mol \% TAG species $=0,50 \%$ ).

The main TAG species in control flies are: 46:1, 48:0, 48:1, 50:1, 52:0 and 52:1. In puml $^{1}$ flies: 48:2, 50:1, 50:2, 52:2, 52:3 (Figure 33, Figure 34). As no total hydrolysis was performed, ratios for the single free fatty acids were not acquired and the individual composition of each TAG species can only be predicted with an amount of uncertainty. Based on the desaturation grade found in the most common lipids and their size (first number represents total number of carbon atoms found in the sidechains of the glycerolipids; second number indicated the number of $\mathrm{C}=\mathrm{C}$-bonds [desaturation grade]) it is very likely that oleic acid (C18:1) and linoleic acid (C18:2) represent the major fatty acids contributing to these TAG species.

Compared to puml ${ }^{1}$ the most abundant TAG species had a shorter length in control flies (Figure 33, Figure 34). In correlation with a higher relative abundance of long 
chain fatty acids (LCFA) also the relative abundance of TAGs with a higher desaturation grade was increased in pum $I^{1}$. Both experimental approaches confirmed a higher abundance of TAGs (in absolute amounts) in Malpighian tubules of pum/ ${ }^{1}$ and complement the data from microscopic analyses and TLC results of lipid extracts deriving from total flies.

The current lipidomics data also strengthens the idea of Puml being involved in the peroxisomal directed $\beta$-oxidation of long chain fatty acids (LCFAs: C13 - C21). Comparable to AtABHD4/5 mutants we have increased abundance of PUFAs and LCFAs in pum ${ }^{1}$. A similar phenotype can also be observed in human patients of the Zellweger syndrome (Suzuki et al., 1996). 

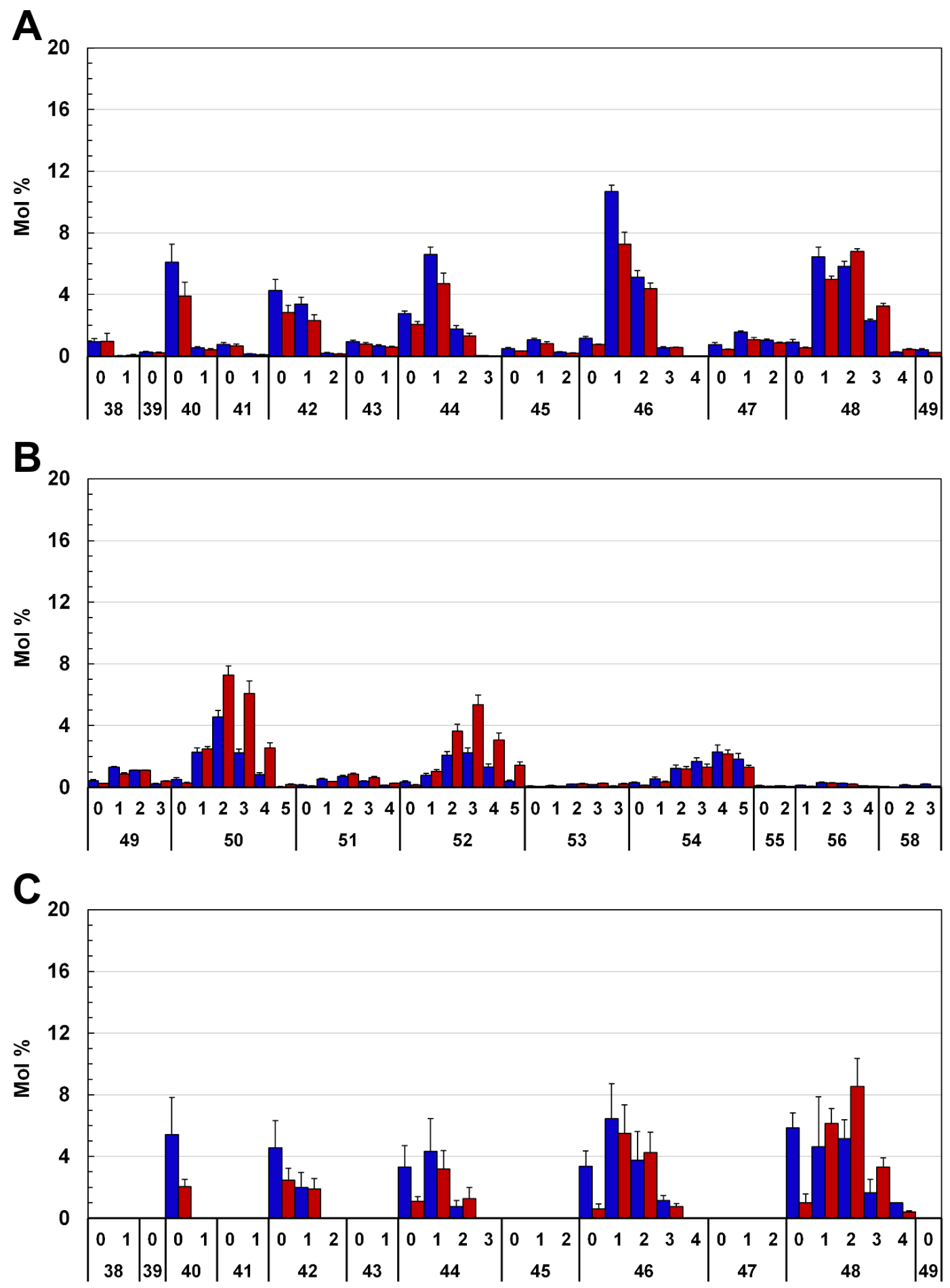


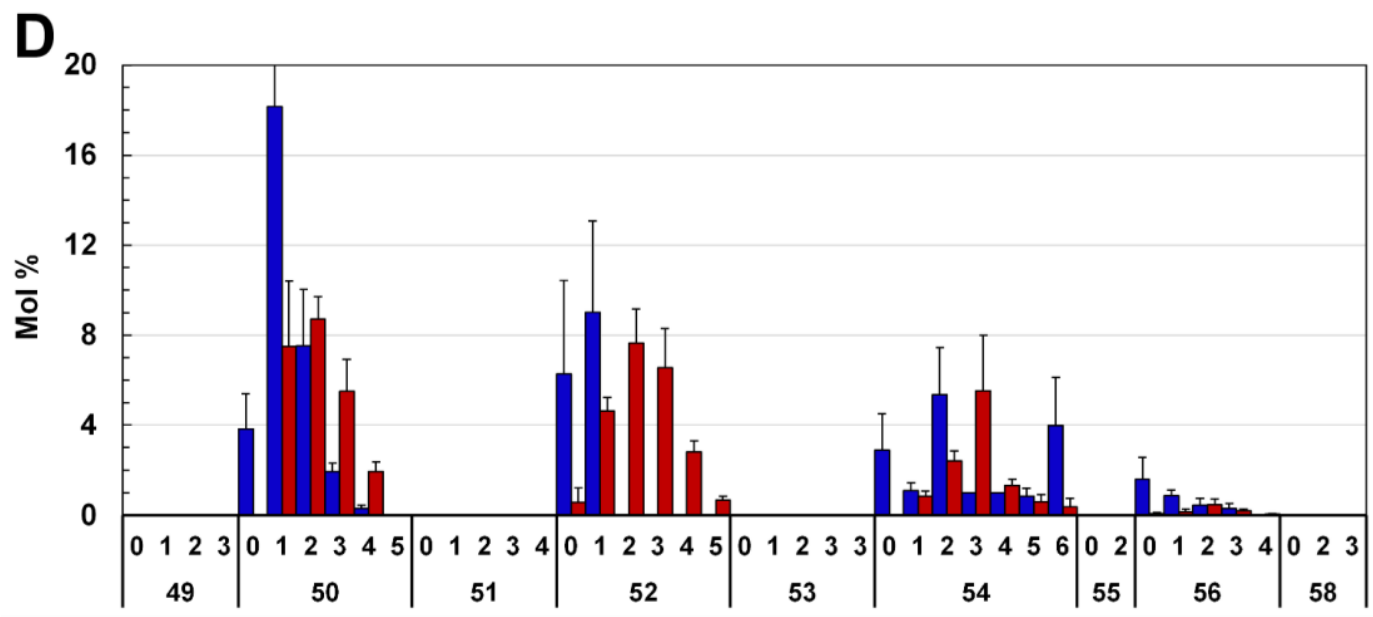

Figure 33 LCFA-TAGs and abundance of PUFAs are elevated in Malpighian tubules of puml ${ }^{1}$ flies. Plotted are the means of Mol \% \pm SEM ( $n=4$ independent extractions) per molecular TAG species (upper numbers represent number of $C=C$ bonds in the fatty acid side chain; lower number the total number of carbon atoms from the esterified FAs; data from control flies are shown in blue; puml ${ }^{1}$ data depicted in red). (A, B) Lipid extraction, mass spectrometry and lipid annotation performed by Vinzenz Hofferek (MPIMP-golm). (C, D) Lipid extraction, mass spectrometry and lipid annotation performed by Dr. Thomas Eichmann (University Graz). Long chain fatty acids (LCFAs) [50, 52] were elevated in pum/ ${ }^{1}$ compared to control flies in both experiments. Additionally, more poly-unsaturated fatty acids (PUFAs) could be detected in puml $^{1}[48: 2,48: 3,50: 3,50: 4,50: 5,50: 2,50: 3,50: 4,50: 5,52: 2,52: 3,52: 4,52: 5)$.

The Zellweger syndrome is characterized by a general loss of peroxisomal functions (Jones et al., 1992). This includes the impaired degradation of mono- and polyunsaturated fatty acids (Christensen et al., 1986, Wanders et al., 1987).

Taken together, in vitro assays revealed no esterase activity for Puml on neutral lipids. In puml ${ }^{1}$ flies body fat is increased and TAGs mostly contribute to this phenotype (Figure 14). Multiple lipidomics analyses confirmed increased TAG storage and revealed PUFAs and LCFAs being more abundant in pum/ ${ }^{1}$. This is very like caused by an involvement of Puml in the peroxisome targeted lipid degradation that at least seems to be decreased in puml ${ }^{1}$ and would explain the dual localization on LDs and peroxisomes.

Additionally, Puml is a potent phospholipase and shows some affinity for PA, PG or $B M P(R, R)$ suggesting a modulating role on the phospholipid monolayer of lipid droplets. This may also provide some explanation why the lipid droplet size distribution is changed in puml ${ }^{1}$ as well. 


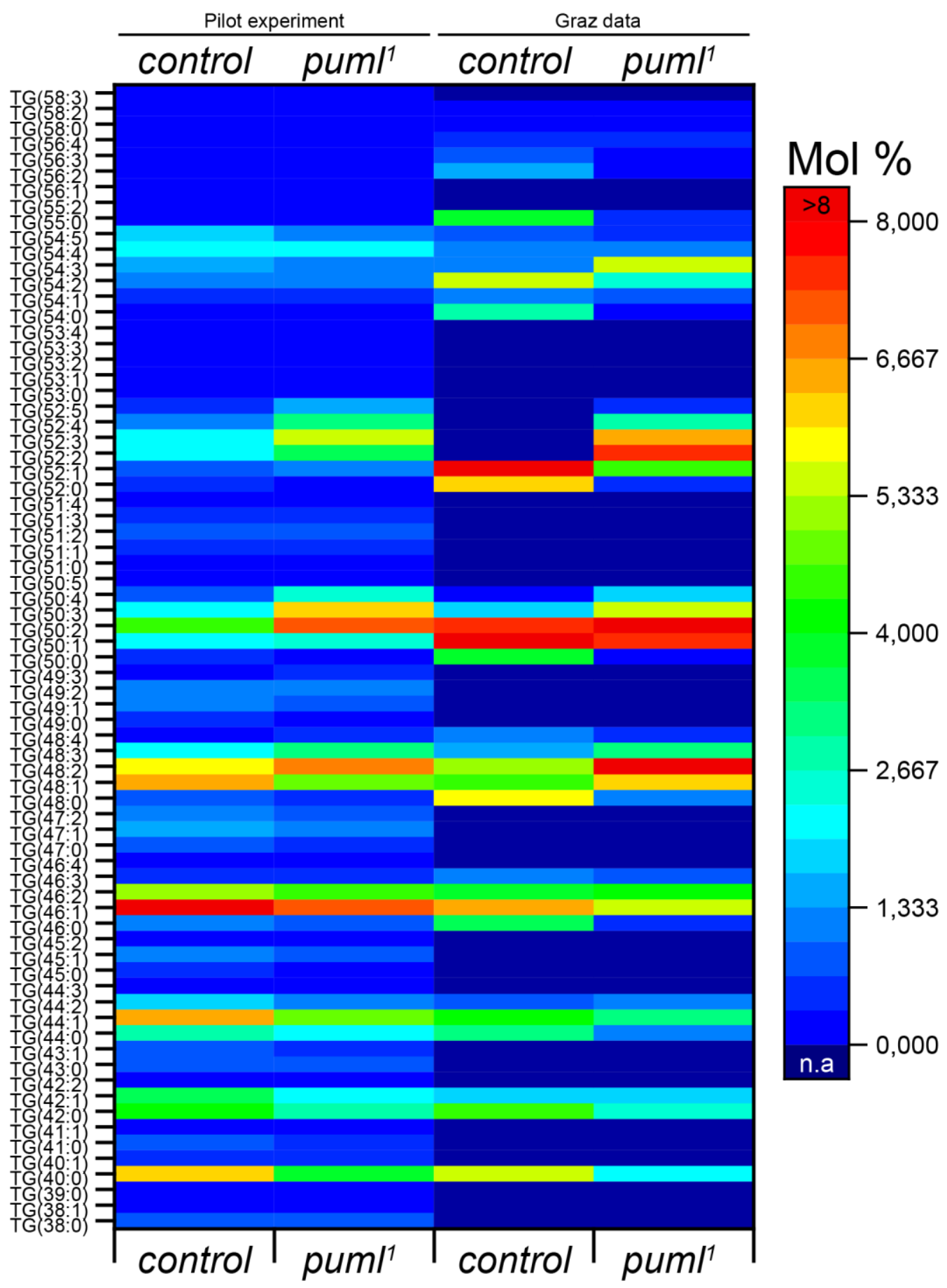

Figure 34 Heat map of TAG species distribution shows increased abundance of PUFAs and shift towards longer fatty acid sidechains in Malpighian tubules from puml ${ }^{1}$ flies. Lipid extraction, mass spectrometry and Lipid annotation done by Vinzenz Hofferek (MPIMP-golm; Pilot experiment; represented are data from 5 discrete extractions from 10 Malpighian tubules [MTs] pairs) and Dr. Thomas Eichmann (University Graz; represented are data from 3 independent extractions from each 100 MT pairs; data with n.a-coloring were not annotated).

Looking at the organismal level absence of puml ${ }^{1}$ is not crucial as homozygous null mutants are viable. On the other hand, carbohydrate stores (glycogen) and lipid stores are modulated differently in puml ${ }^{1}$. Whereas food intake is not impaired in 
puml ${ }^{1}$, ingested food is preferentially stored in form of TAGs to the account of carbohydrates. This may contribute to the findings that puml ${ }^{1}$ have a higher starvation resistance but are more susceptible to desiccation.

Lipid mobilization in general is not impaired in pum ${ }^{1}$ and a direct enzymatic activity on neutral lipids could not be detected. Therefore, the biological role of pum/ seems to be more indirect in assisting lipolysis, directing fatty acids and acting more like a metabolic switch at the junction between carbohydrate and lipid metabolism in Drosophila melanogaster. This would also characterize the core lipid mobilization module of ABHD5-ATGL more as a specialization during evolution providing an additional level of control and enhanced efficiency of lipid mobilization in higher organisms rather than an evolutionary conserved theme. 


\subsection{Localization, interactions and structure of Pummelig}

High throughput expression pattern data for puml shows that different isoforms are expressed throughout all developmental stages (Gelbart and Emmert, 2013). Whereas they differ in total length, they can be separated into two group that share the core $\alpha / \beta$-hydrolase domain. The main difference is variable length of their $\mathrm{N}$ termini. Experiments from Anna Takacs (Takacs, 2007) indicated differences in the localization of mCherry-tagged overexpression of the long (Puml-PA) and the shorter (Puml-PB) protein. Both tagged proteins exhibited a dot-like pattern in the cytoplasm. Whereas Puml-PA::mCherry additionally localized on lipid droplets the shorter version (Puml-PB::mCherry) did not. This might indicate that the longer $\mathrm{N}$-terminus is needed for the LD association. Indeed, recent findings for ABHD5 showed that the $\mathrm{N}$-terminal region is crucial for LD binding (Gruber et al., 2010). Another study revealed that a small Tryptophan-rich arm mediates the interaction with the phospholipid monolayer (Boeszoermenyi et al., 2015). Interestingly, a more detailed analysis of the Puml isoforms (Figure 11 ) showed, that the longer N-terminus contained multiple phenylalanine $\left(F^{52,53}\right)$ and tryptophan $\left(W^{55,57,61}\right)$ residues implying a comparable lipid-anchor motif in Puml. This might explain the different localization pattern of the Puml::mCherry isoforms as only Puml-PA:;mCherry with the long $\mathrm{N}$ terminus can be found on lipid droplets.

Puml shares the $\alpha / \beta$-hydrolase domain with ABHD4 and 5 , but in contrast to ABHD5 the catalytic center (GxSxG motif) is active in Puml. Additionally, enzymatic experiments revealed that Puml does not stimulate the lipase activity of Brummer on triglycerides indicating that the lipid mobilization core module ABHD5-ATGL is not evolutionary conserved. As the $\alpha / \beta$-hydrolase domain contains two active catalytic sites (GxSxG and HxxxD) Puml may modulate lipid metabolism in a different way. Perhaps, part of the function of puml depends on its localization as the different isoforms (tagged mCherry constructs) appear to have different localization patterns. By this Puml may has a different function on lipid droplets than on peroxisomes. 
As ABHD5 orthologues in Arabidopsis thaliana (James et al., 2010), Saccharomyces cerevisiae (Ghosh et al., 2008a) and C. elegans (Xie and Roy, 2015) are also known to modulate lipid metabolism and have active catalytic sites its absence in ABHD5 might be a relatively new evolutionary adaption. Also, a forward-mutation (A155S) to reconstitute the active GXSXG-motif in ABHD5 does not resemble the lipolytic activity (Wang et al., 2011). This indicates that perhaps additional structural changes may occurred in ABHD5 leading to a diversion from the ancestral mechanism through which ABHD5 regulates lipid metabolism. On the other hand, the biological function of ABHD5 does not rely exclusively on the interaction with ATGL as ATGL knock out mice do not exhibit lipid accumulations in the skin [ichthyosis] (Lefevre et al., 2001). Additionally, ABHD5 knock out mice exhibit severe defects in skin permeability, ectopic lipid storage, hepatic steatosis and decreased acyl-ceramide production leading to early death after birth (Radner et al., 2010). This indicates that ABHD5 has an ATGL independent function with an up to now unknown mechanism.

It is known, that ABHD5 interacts with PLIN1, PLIN2, PLIN5, ATGL and FABP (Yamaguchi et al., 2004, Lass et al., 2006, Granneman et al., 2009, Hofer et al., 2015). For this interaction the $\mathrm{C}$-terminus was identified to mediate this protein-protein interactions (PPI). A single point mutation (E262K) eliminates the interaction with PLIN1 and PLIN2 (Yamaguchi et al., 2004, Yamaguchi et al., 2007). The region of the first and second $\alpha$-helix loop is important for the binding do FABP (Hofer et al., 2015).

The overexpression of Puml-PA::mCherry in Drosophila free floating adipocytes from freshly hatched $p / i n 1^{1}$ flies leads to reversion of the giant lipid droplet phenotype of plin1 ${ }^{1}$ (Takacs, 2007) indicating that Plin1 is not crucial for LD binding of Puml but does not exclude a possible interaction. However, the reduced lipid storage in six-day old flies expressing Puml::mCherry in the fat body of plin1 $1^{1}$ flies (Takacs, 2007) suggests at least a possible negative effect on a lipolytic function of Puml that may rely on the interaction of Plin1 and Puml.

On the other hand, Puml was found on embryonic lipid droplets (Cermelli et al., 2006) a developmental stage where Plin2 is predominantly expressed compared to Plin1 (Gelbart and Emmert, 2013). As Plin2 is more abundant on LDs in plin $1^{1}$ larvae (Sahu- 
Osen, 2015), implying a redundant role of Plin2 to Plin1, Puml may interacts with this member of the perilipin family. However, plin $1^{1}$ plin $2^{1}$ double knockout flies exhibit very low body fat storage after hatching that partially recovers to the levels of plin $2^{1}$ flies, which still have a $\sim 40 \%$ reduced body fat storage than control flies, indicating that these flies might be lipolytically more active like $b m m$-gof flies that are also store less body fat (Figure 13).

puml-gof flies showed no decrease in body fat storage, which is congruent with data from mice overexpressing ABHD5 (Caviglia et al., 2011), also (Figure 13). On the other hand, upon puml-gof in puml ${ }^{1}$ flies the over-storage phenotype could be reverted to normal levels. This argues rather in favour of pum/ modulating lipid storage indirectly and not by actively participating in neutral lipid breakdown.

Interestingly, whereas the expression of puml in pum ${ }^{1}$ flies reverted the body fat over-storage this could not be achieved by the expression of Puml-PA::mCherry. Although Puml-PA::mCherry localizes on lipid droplets and peroxisomes, preliminary data shows that body fat storage and ectopic lipid storage in Malpighian tubules remained unchanged [Figure 35].
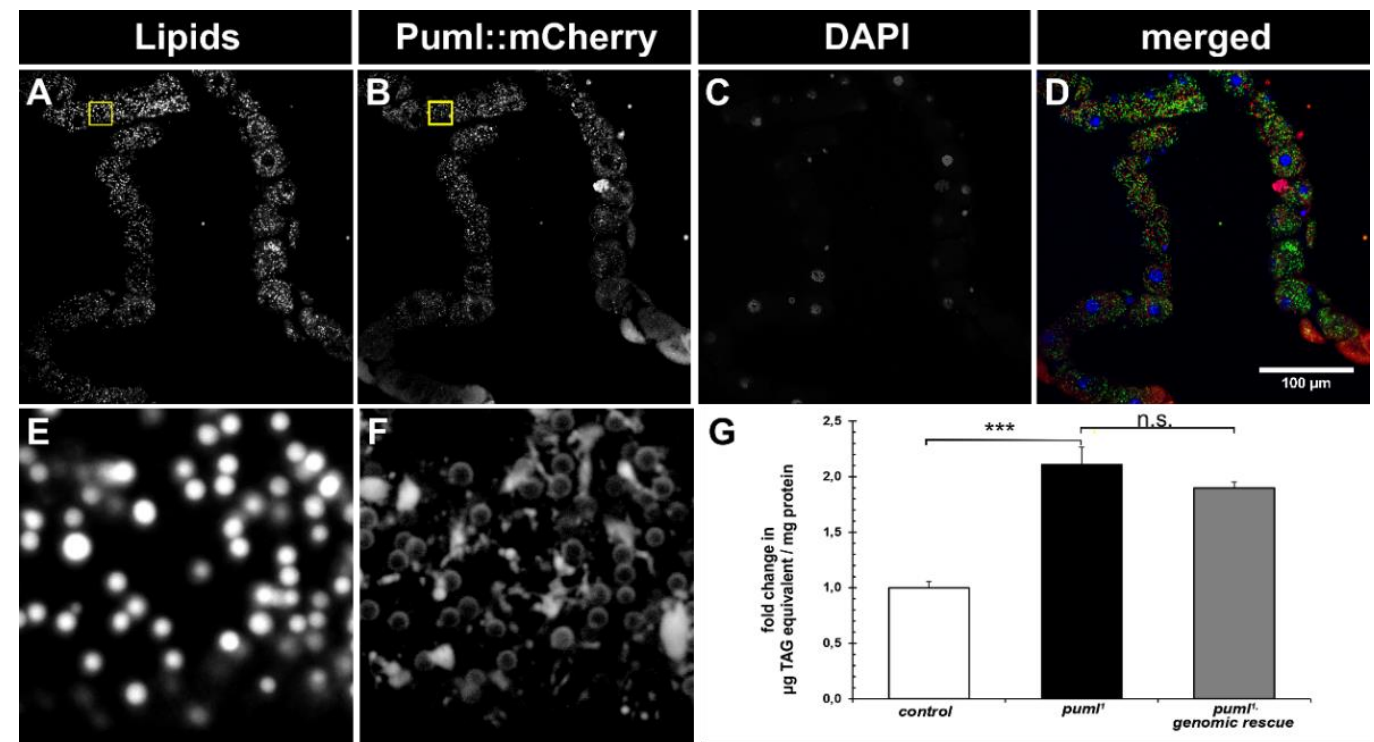

Figure 35 A genomic rescue of puml with Puml::mCherry does not reduce the amount of body fat to control flies and does not improve ectopic lipid storage. Lipids (stained by Bodipy493/503) can be still found in malpighian tubules ( $A, E$ [magnified box from $A]$ ) with expressed Puml::mCherry ( $B, F$ [magnified box from B]). DAPI was used to stain nuclei (C) and the merged channels (D) show a localization of Puml::mCherry on lipid droplets. A magnified picture shows the ring-like pattern $(F)$ of Puml::mCherry that match with stained lipid droplets (E). Body fat storage (G) was still increased in the genomic rescue flies (average fold change in $\mu \mathrm{g}$ TAG equivalent /mg protein \pm SEM; Mann-Whitney test, $* * *=P<0.001)$. 
As the C-terminal localization signal for peroxisomes (SKL-motif) is absent in Puml, this supports the theory of a needed interaction partner for the localization to peroxisomes, which remains to be identified but this possible interaction is not prohibited by the C-terminal mCherry-tag. However, the modulation of lipid storage seems to be impaired in the fusion protein. This might be due to structural inaccessibility caused by the mCherry-tag shielding a binding site for other proteins or possible phosphorylation sites. An in silico analysis predicted 27 potential phosphorylation sites that were enriched at the C-terminal end of Puml (Figure 11). As phosphorylation partially modulates the function of ABHD5, that is a phosphorylation target of PKA (Ser239) (Sahu-Osen et al., 2015), this posttranslational protein modification might be impaired in Puml-PA::mCherry limiting its full biological function. However, the PKA consensus-sequence: RKYS ${ }^{239} S^{240}$ is only partial conserved in Puml (RKFQS) this activation behaviour might be not evolutionary conserved. On the other hand, this phosphorylation site is also not conserved in ABHD4 (Figure 10) supporting a different mechanism by which puml is modulating lipid storage.

Thus the identification of possible binding partners is of high importance to elucidate the biological function of puml in lipid metabolism. For this, it might be crucial to obtain a functional antibody to detect endogenous Puml as $\mathrm{N}$-terminal tags might interfere with the lipid droplet localization and C-terminal tags may prohibit, needed post-translational modifications or impair protein-protein interactions with Puml directly. Also, an antibody could be used to verify the localization of Puml::mCherry. An approach (in this work) that covered the generation of several monoclonal antibodies against different peptides from Puml did not produce a useable antibody to detect endogenous Puml (data not shown).

\subsection{Enzymatic activity of Puml}

Based on the similarity to ABHD5 it was assumed that Puml may activates Bmm lipase. However, in vitro assays provided no evidence for Puml stimulating the TAG hydrolysis activity of Bmm. On the contrary, comparable to the mammalian ABHD4 or orthologues in plants (James et al., 2010), yeast (Ghosh et al., 2008b) or nematodes 
(Ashrafi et al., 2003) also Puml modulates lipid storage and has an active catalytic center (GXSxG-motif) (Figure 10). Therefore, a substrate screen was performed to find potential targets for Puml. The Substrate screen identified Puml as an active phospholipase (PA, PG, BMP[R,R], NAPE) with a very weak affinity for MAG, that works preferentially in physiological to basic $\mathrm{pH}$. This hydrolase activity measured by the quantification of the released non-esterified fatty acids (NEFAs) was determined by the activity of both catalytic sites as preliminary data for each single knock out showed a total loss of activity (Figure 36).

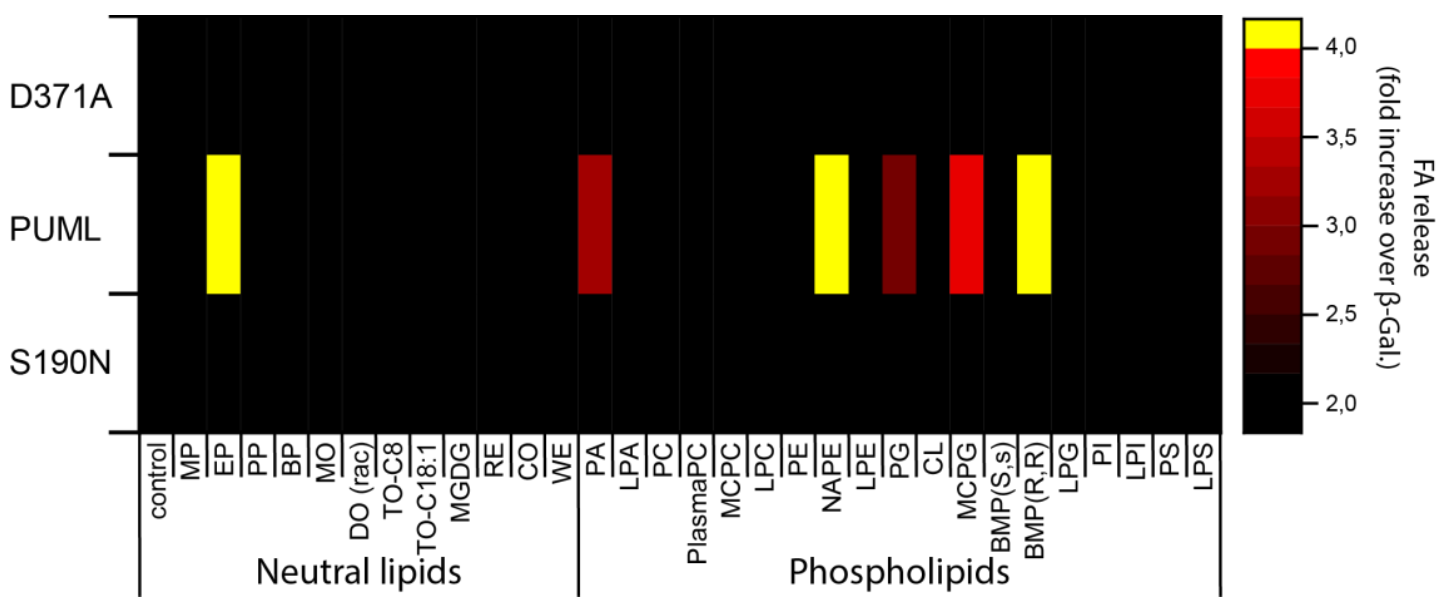

Figure 36 Preliminary data shows that a point mutations in each single catalytic site of Puml results in a total loss of enzymatic activity for the tested substrates of the screen. Puml wildtype and point-mutants were expressed in insect cells for this screening. Shown are the fold changes in FA release normalized to the B-Galactosidase (negative control). A fold change of 2 was used as a lower threshold. The qualitative measurement reveals that the point mutations PumI-PA:S19ON and PumlPA:D371A both abolish the enzymatic activity of wildtype Puml-PA.

Knowing possible targets for Puml raised the question what biological relevance these potential activities might have. A possible explanation for the increased body fat storage of pum/ ${ }^{1}$ flies was a possible impairment of the lipolytic capability but the time course experiment revealed that the neutral lipid mobilization actually occurred faster in pum $l^{1}$ flies.

Endocannabinoid signaling is known to influence energy metabolism (Panakova et al., 2005) and exists in Drosophila (Tortoriello et al., 2013, Khaliullina et al., 2015). As it is known that ABHD4 is involved in the generation of $\mathrm{N}$-acylethanolamines (NAEs) from $\mathrm{N}$-acylphosphatidylethanolamines (NAPEs) it might be possible that Puml serves a similar function in the fly hydrolysing NAE precursors. However, data in puml ${ }^{1}$ larvae exhibited no drastic changes in NAE levels. Besides a small significant reduction in 
NAE C18:2 by $\sim 40 \%(P<0.05)$ no changes could be seen for NAE C16:0, C18:0 and C18:1 (data from a pilot experiment, personal communication from Tomasz Buhl; Eaton lab; MPI-CBG: Dresden). However, these experiments were performed in puml ${ }^{1}$ larvae and only for the four most abundant NAEs were analysed. Therefore, it might be possible that Puml has a specificity for other NAPEs.

Taken together, Puml is not necessary for the generation of the most abundant NAEs in larvae; however, NAE levels were not analysed in adults. Thus, it cannot be excluded that Puml may is involved in NAE synthesis in adult flies. Nevertheless, this should be investigated further as NAEs are involved in various biological processes in mammals like food intake, immune- and inflammatory response as well as energy metabolism (Heier et al., 2016).

Bis(monoglycero)phosphate (BMP[R,R], sn-(3-oleoyl-2-hydroxy)-glycerol-1-phosphosn-1'-(3'-oleoyl-2'-hydroxy)-glycerol (ammonium salt) is a known substrate of mammalian ABHD6 (Pribasnig et al., 2015) an enzyme that can act as a MAG lipase and is affiliated with endocannabinoid signalling. More specifically BMP is enriched in late endosomes/ lysosomes where it is crucial for lipid sorting and the formation of intraluminal vesicles (Pribasnig et al., 2015). With an overlap in the substrate specificity (MAG, $B M P(R, R)$ ) Puml may serves a similar role in Drosophila in directing lipid catabolism. To show a possible association lysosomes/endosomes additional localization studies with Puml are needed. Since Puml localizes partially to peroxisomes and majorly to LDs these organelles might represent an additional resting site.

Interestingly, the affinity for $\mathrm{BMP}(\mathrm{S}, \mathrm{S})$ was very low, indicating a strong steric preference for its stereoisomer. Besides this Puml also exhibits hydrolase activity for the structural isomer of BMP: Phosphatidylglycerol (PG) with long (C18) and medium (C12) fatty acid sidechains (Figure 20). Also Puml can hydrolase Phosphatidic acid (PA). However, PA and PG do not represent the most abundant phospholipid species in Drosophila. The most abundant phospholipids in yeast and mammals are Phosphatidylcholine (PC) with $50-60 \%$ followed by Phosphatidylethanolamine (PE) (20-30\%) whereas in fruit flies it is switched (PE 50-60\% followed by PC 20-25\%) 
(Tauchi-Sato et al., 2002, Bartz et al., 2007, Carvalho et al., 2012, Guan et al., 2013). This is of interest as Puml is localized on LDs and puml ${ }^{1}$ flies have decreased LD size in malpighian tubules. Therefore, Puml might be involved in the regulation of the phospholipid monolayer finally defining the physiochemical parameters of LDs and by this defining its size and maybe accessibility by proteins. However, modulation of PC but not PE is highly critical for LD size (Krahmer et al., 2011). This might be supported by the fact that RNAi mediated knockdown of CG1882 in S2-cells revealed no lipid droplet phenotype (Guo et al., 2008) but it could be possible that this LD size phenotype is restricted to the adult stage. Of course, in order to show a changed phospholipid composition on LDs a lipidomics analysis should be performed on purified LDs from adult pum/ $I^{1}$ flies.

Taken together, Puml exhibits phospholipase activity and several potential targets could be identified in a substrate screen. However, it is not clear if Puml actually hydrolysis these substrates in vivo and how this missing activity contributes finally to increased storage of TAGs.

\subsection{Lipogenesis in pummelig mutants}

As shown in this work puml ${ }^{1}$ adult flies have increased body fat storage and exhibit ectopic lipid storage as well. One possible explanation was that this was caused by an impaired capability to mobilize storage lipids like in $b m m^{1}$. However, this is very unlikely as puml ${ }^{1}$ flies could mobilize their storage lipids.

Another theory was that the increased TAG storage might be caused by increased lipogenesis in puml ${ }^{1}$ flies. Indeed, lipogenesis was increased in pum $I^{1}$ flies (Figure 21). More interestingly, this was not due to an increased food intake but more likely a redirection from ingested glucose into storage lipids as glycogen stores were decreased in puml $l^{1}$ (Figure 23).

Especially the significant differences in phospholipids after the chase periods might be interesting as they may indicate an imbalance in the phospholipid metabolism of puml $^{1}$ as well. 
However, the experiments performed on lipogenesis do not allow to draw conclusions about the turnover of lipids. Probably, this should be addressed in the future as neutral lipid storage disease (NLSD) in mammals is characterized by an abnormal glycerolipid metabolism (Igal and Coleman, 1996) and a higher synthesis and turnover of phospholipids. Though, pum/ ${ }^{1}$ flies may serve as an animal model system for NLSD to find therapeutics to treat this disease.

Additional experiments should also focus on the actual nutrient resorption from the food using labelled substrates as pum ${ }^{1}$ flies may consume comparable amounts of food but utilize them more efficiently than control flies and therefore have a higher caloric loading from the food. As endocannabinoids are known to interfere with the microbiome in the gut of mice (Muccioli et al., 2010) maybe a possible impairment in this signaling in puml ${ }^{1}$ shapes it towards a direction that allows a more efficient nutrient resorption also in flies.

A possible involvement of puml for lipogenesis on the other side so far remains puzzling. A possible reason for the increased generation of TAGs may can be explained with the PA hydrolase activity of Puml as PA is a precursor for various phospholipids and DAG (Igal and Coleman, 1996) that is needed for the generation of TAGs. As it is known in other organisms that high levels of PA (Toschi et al., 2009, Brown et al., 2010) promote the generation of TAGs, may locally increased amounts of PA in puml ${ }^{1}$ might be directed into TAGs as well. Whether PA levels are elevated in puml $^{1}$ is not known and should be addressed in the future. Interestingly, it has been shown that specific PA species (C18:0 and C18:1) and PGs are elevated in ABHD5 knock down mice (Brown et al., 2010). However, the mechanism of PA generation in mice based on the Lyso-phosphatidic acid acyl-transferase (LPAAT) activity of ABHD5 (2012a). LPAAT activity was first described in 2010 (Montero-Moran et al., 2010) but rejected in 2014 (McMahon et al., 2014a). Therefore, it remains open how PA levels in ABHD knock down mice were changed.

As PA stabilizes dTOR (Foster and Toschi, 2009, Toschi et al., 2009) which is known to regulate energy homeostasis by negatively modulating the expression of $\mathrm{bmm}$ and dilp2 and dilp5 lower basal lipolytic activity may contributes to the increased amount 
of storage lipids found in $p u m I^{1}$. It is also known for PA to negatively regulate AMPK a critical cellular energy sensor (Hardie, 2011, Mukhopadhyay et al., 2015) that modulates also autophagy, mitochondrial biosynthesis and $\beta$-oxidation. Therefore, increased amounts of PA in puml ${ }^{1}$ flies could lead to a lower activity of AMPK. Subsequently, the negative regulation of Acetyl-CoA-carboxylase (ACC) by AMPK would be absent that would finally enhance the generation of Malonyl-CoA from Acetyl-CoA in the cells. As high amounts Malonyl-CoA is an intermediate substrate for lipid synthesis and inhibits CPT1, the rate limiting enzyme for the transport of long chain fatty acids (LCFAs) into mitochondria, beta-oxidation would be negatively regulated also on another level. This would also provide an explanation for the increased amounts of LCFAs and very long chain fatty acids (VLCFAs) found in pum/ ${ }^{1}$ flies.

Taken together the PA hydrolase activity of Puml might be important for the regulation lipogenesis by lowering the concentration of this intermediate substrate that is used for the synthesis of TAGs and by this avoiding high amounts of PA that could lead to an increased TOR signaling and lower AMPK signaling in puml ${ }^{1}$. By this, puml would provide an additional mechanism antagonistic to insulin signaling establishing a negative feedback loop to balance lipogenesis and lipolysis in the cells. Interestingly, many tumours exhibit suppressed AMPK- and increased TOR signalling, which enables cancer cells to avoid mitochondrial ATP generation and shift the metabolism towards cytosolic glycolysis (Warburg effect) (Jones et al., 2005, Faubert et al., 2013). As ABHD5 has been described as an anti-cancer gene (Ou et al., 2014) maybe this principle mechanism is evolutionary conserved. A knockdown of ABHD5 but not ATGL lead to decreased phosphorylated AMPK levels, increased amounts of pAKT and lower amounts of p53 in mammalian cells (SW620 and HCT116 cells) (Ou et al., 2014). This lead in ABHD5 knock down cells to a higher glucose disposal (Brown et al., 2007) and lower fat utilization that was accompanied by a higher fat storage in these cells as well. However, Ou et al. (Ou et al., 2014)could not provide a direct mechanism by which ABHD5 absence was contributing to the cellular alterations but postulated an ATGL independent function of ABHD5. In agreement with the finding that p53 was involved in mediating the metabolic shift in mammalian cells towards 
glycolysis a conserved mechanism could be identified in flies (Barrio et al., 2014). Dp53 is regulated by dTOR via miR-305 and insulin signaling. Under feeding conditions high nutrient availability leads to the upregulation of miR-305 and increased insulin signaling that inhibits generation of p53 and fosters p53 degradation. Through this pathway, dTOR activity is enhanced by a positive feedback loop. As high dTOR signaling leads to decreased CAMP generation PKA stimulated lipolysis is decreased. Additionally, high levels of activated PKB/Akt lead to a greater inhibition of Glycogensynthase by phosphorylation through GSK3 $\alpha$ and thereby decreased glycogen storage in mammals. This mechanism is conserved in flies where GSK-3 (shaggy) is negatively regulated during insulin-like signaling and limits glycogen synthesis (Papadopoulou et al., 2004). As pum/ ${ }^{1}$ flies have decreased glycogen storage maybe cells experience a state of hyper-activated insulin-like signaling that leads to inhibition of glycogen synthesis and simultaneously to increased TOR signaling resulting in decreased lipolysis under basal conditions and enhanced lipogenesis (Figure 37).

In order to be able to adapt to constantly changing environmental conditions also the energy storage and mobilization needs to be regulated very precisely. The underlying mechanisms are highly complex and involve several extracellular to and intracellular signals in the fat body. Therefore, the proposed indirect mechanism by which puml could modulate lipid metabolism might work on regulatory level at the cellular level rather than actively participate in the hydrolysis of storage lipids. The current model can explain the decreased glycogen levels and increased TAG storage observed in puml ${ }^{1}$ flies. 


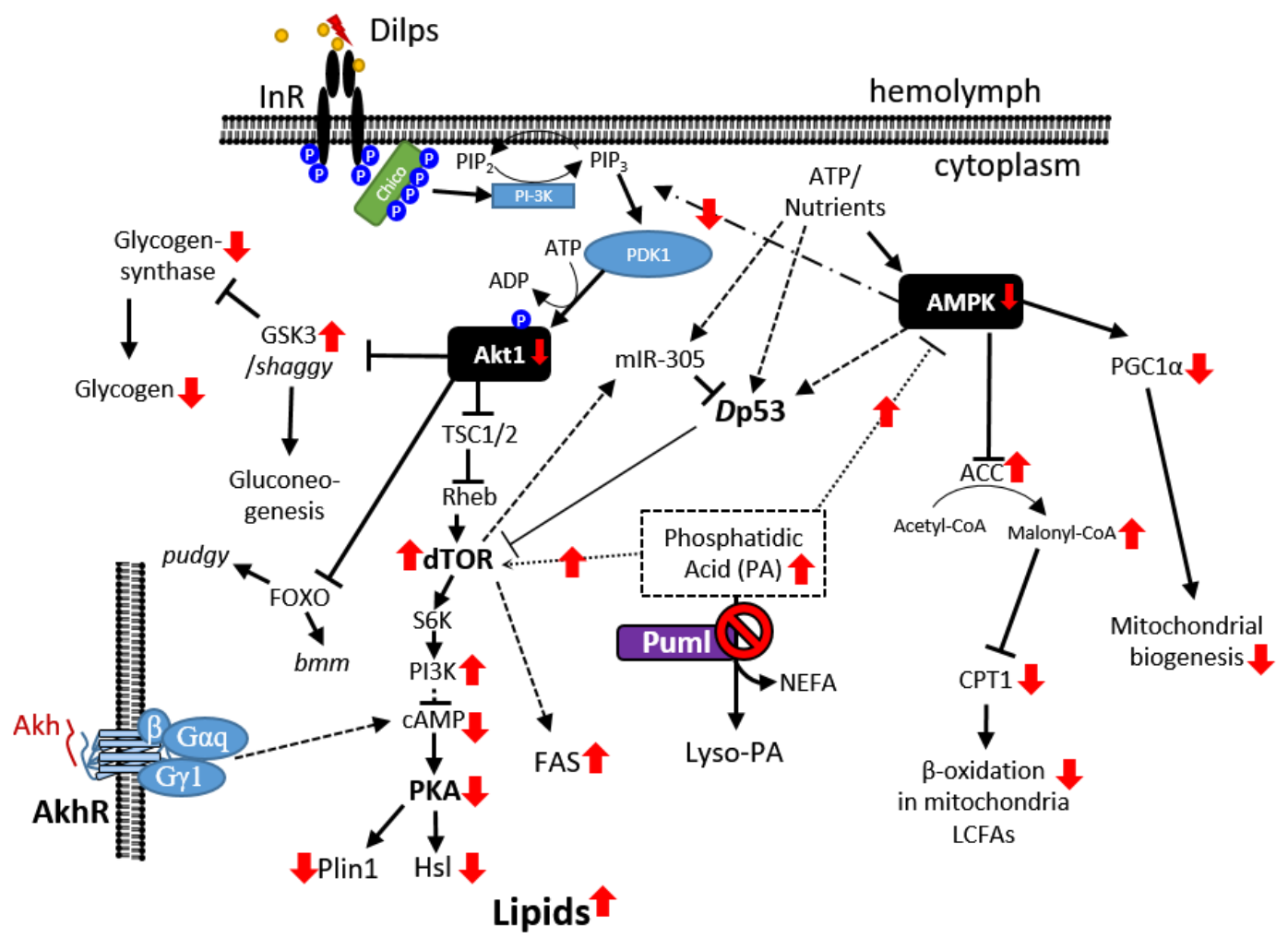

Figure 37 Current model of puml regulating lipid storage in flies. Signalling pathways and their connection were depicted based on literature data (black). Regulation during energy expenditure/starvation are shown in black. Red labelling indicates possible effects of puml deficiency in adult flies under fed conditions.

The protective role of obesity against the harmful effects of a high sugar diet has been addressed recently (Musselman et al., 2011). The study showed that the metabolic fate of glucose was reprogrammed in flies under a high sugar diet (HSD) regime and in lean flies. Based on the finding that a high sugar diet leads to increased blood sugar and systemic insulin resistance in developing larvae and adult flies (Musselman et al., 2011) they followed the carbon flux using a stable isotope tracer $\left({ }^{13} \mathrm{C}\right.$-glucose). Indeed, storage fat synthesis from dietary glucose was lower in larvae raised on HSD and TAG species shifted towards shorter esterified fatty acids and contained more unsaturated fatty acids (Musselman et al., 2013). Conversely, larvae on HSD exhibited increased amounts of NEFAs. Consistent with this finding lipogenic genes like Mio (CG18362) and desat1 (CG5887) and $\beta$-oxidation genes (e.g. DmCPT1 or ACS) were upregulated under HSD. In order to generate lean fat body tissue they performed a knockdown of mio which encodes a protein homologues to carbohydrate-responsive transcription factor (ChREBP) in mammals (Postic et al., 2007). Consistently, a knock 
down of Mio (cg-GAL4) caused a decreased expression of fatty acid synthase (FAS), lipid storage modulators like bmm, plin1 or plin2 and genes involved in insulin signaling (chico, Tor, Akt, S6K, PDK, PI3K). This lead to decreased storage lipid mobilization und insulin resistance in cg-Gal4>mio-RNAi wandering larvae on a HSD. Interestingly, expression of several lipases but not $\mathrm{bmm}$ were upregulated, as well as whd (DmCPT1) and genes involved in Ketone metabolism. As a result, carbon flux was channelled towards generation of NEFAs through ketones and improved $\beta$-oxidation in mio knock down larvae on a HSD.

Surprisingly, puml was upregulated in $c g-G a l 4>M i o-R N A i$ wandering larvae on HSD (Musselman et al., 2013) indicating a different function for this protein in modulating lipid metabolism. Consistent, with the current working model (Figure 37) pum/ might be repressed by mio. This regulation would lead to a decreased expression of puml during increased carbohydrate load of fat body cells mimicking the effects of pum/ ${ }^{1}$ flies leading to increased generation of storage lipids and increasing insulin sensitivity of the fat body. On the other hand, the upregulation of puml in mio knock down larvae challenged by a caloric overload of sugar (HSD) would improve AMPK signaling leading to increased mitochondrial biosynthesis and decreased inhibition of DmCPT1 by Malonyl-CoA (AMPK inhibits ACC) but would not improve the insulin insensitivity observed in larvae with a mio knock down in the fat body on a HSD.

\subsection{Lipolysis}

NLSD is characterized by the imbalanced phospholipid metabolism, increased TAG storage but normal lipolysis rate (Igal and Coleman, 1996). Igal and colleagues favoured a mechanism by which generated DAG during lipolysis was majorly utilized for re-synthesis of TAGs. As shown before puml ${ }^{1}$ share some characteristics with NLSD patients like increased storage of TAGs, also ectopically and lower glycogen storage. Therefore, one theory of the increased body fat storage was based on the increased lipogenesis in puml ${ }^{1}$ flies that could be shown in this work. However, the second possibility was an impairment in the storage lipid mobilization in puml ${ }^{1}$ flies. 
Apparently, lipid mobilization in pum/ ${ }^{1}$ flies was not lower but actually higher during nutrient deprivation. Metabolic rate measurements revealed no differences between puml ${ }^{1}$ and control flies (Figure 22) but as on oxygen consumption was quantified and not exhaled carbon dioxide no assumptions for the major catabolic substrate can be made from the respiratory quotient. Though, Glycogen and lipids are mobilized simultaneously in the flies until glycogen stores were completely depleted (Figure 23). Probably additional respirometric measurements should be performed on pum $I^{1}$ flies to identify the main carbon source for energy synthesis under fed conditions and during starvation. Though, increased TAG storage in puml $^{1}$ seems to be the main cause for the increased starvation resistance compared to control flies.

One possible reason of the increased lipolysis during starvation might be reduced Glycogen storage in puml ${ }^{1}$ flies. It had also been described that high amounts of nonesterified fatty acids (NEFAs) can impair glucose utilization leading to the so called Randle effect (Randle et al., 1963, Ussher and Lopaschuk, 2009). So far it has not been shown whether basal lipolytic activity in puml ${ }^{1}$ is changed as well as it would provide an additional explanation why glucose metabolism is changed in puml ${ }^{1}$ flies. So far, TLC data (Figure 14) provided no evidence for increased amounts of FAs in puml $l^{1}$ flies. NEFAs are detected by HNF4 (Drosophila ortholog to mammalian PPAR $\alpha$ ) that leads to increased expression of peroxisomal and mitochondrial genes and therefore allowing a higher fatty acid oxidation (Palanker et al., 2009). Therefore, also basal lipolysis might be increased in pum/ ${ }^{1}$ flies leading to higher availability of NEFAs that could be sensed by HNF4.

This could lead to an increased capability of lipid catabolism that might be not used due to an increased re-synthesis of TAGs in puml ${ }^{1}$ and missing activation of hydrolysed fatty acids by pudgy (Drosophila Acetyl-CoA synthetase [ACS]). As pudgy ${ }^{1}$ flies have increased fat storage, decreased Glycogen stores, exhibit lower dilp (dilp2 and 5) expression and are hyperglycemic this might be an explanation for the higher lipolysis during starvation (Xu et al., 2012). As pudgy is a transcription target from $\mathrm{dFoxo}$ and therefore underlying gene suppression by insulin-like signalling may provide an additional argument for the elevated TAG storage in pum/ ${ }^{1}$ flies. 
Also pudgy ${ }^{1}$ flies exhibited an increase of many but not all TAG species with a preference for longer acyl-chains and higher unsaturation grade a phenotype that has some similarity with puml ${ }^{1}$ flies were LDs in Malpighian tubules accumulated TAGs with longer side-chains and had a higher abundance of PUFAs.

However, besides increased lipid mobilization in pum ${ }^{1}$ a significant amount remained un-mobilized in pum $I^{1}$. Whether these lipids are mobilization resistant or remained due to the general higher abundance storage lipids in pum/ ${ }^{1}$ flies and the possibility that lipids alone are not the only limiting factor during starvation remains to be answered. A starvation experiment with the corresponding analysis of lipid content in early dying animals and the best $5-10 \%$ of survivors of starvation might provide a better insight if some lipids cannot be utilized in puml ${ }^{1}$. With the localization of Puml on peroxisomes it is very likely that these "mobilization resistant" lipids then would have LCFAs or VLCFAs esterified to the glycerol and might have a higher unsaturation grade due to the delimited metabolism through peroxisomes.

\subsection{Global fat storage role of puml}

In summary, pum ${ }^{1}$ flies have increased body fat storage in the form of TAGs and lower Glycogen stores. This is rather caused by increased lipogenesis than inhibited storage lipid mobilization. As pudgy ${ }^{1}$ flies exhibit a similar phenotype this protein might be potential binding partner for Puml [pudgy is localized on mitochondria (Xu et al., 2012)]. However, hyperthrehalemia observable in pudgy ${ }^{1}$ should be also addressed in puml ${ }^{1}$. On the other hand, conversely to puml ${ }^{1}$ flies, pudgy flies are long lived and have a slightly reduced body size a phenotype associated with decreased insulin signaling ( $\mathrm{Xu}$ et al., 2012). However, the tight transcriptional regulation of Pudgy might explain the effect that a puml-gof does not lead to decreased body fat storage.

In general, the lipid routing might be impaired in pum $l^{1}$ flies. Lipidomics analysis from malpighian tubules revealed an enrichment in PUFAs and LCFAs and VLCFAs. As these enriched lipids are degraded via the peroxisomes this pathway is at least mitigated in pum $I^{1}$ flies. It remains to be shown if there actually are TAG species in puml ${ }^{1}$ that are mobilization resistant. Aside with the observation that Puml::mCherry is also 
associated with peroxisomes at least supports the model of puml in aiding in the channelling/sorting of lipids. Interestingly, a ABHD5 knock down in hepatocytes exhibits a similar phenotype with hepatic steatosis (lipid accumulation in the liver) accompanied with an accumulation of PUFAs and VLCFAs. At the same time the ABHD5 knock down prevented high fat diet induced obesity in these mice and improved the global glucose tolerance and increased insulin sensitivity (Lord et al., 2012). However, the cellular mechanism by how these changes derive might be different between vertebrates and flies. Interestingly, at the same time ABHD5 knock out adipose tissue had decreased lipogenesis and lysates exhibited lower in vitro Triglyceride hydrolase activity. This very likely is caused by the absence of ABHD5 stimulating function of ATGL in this tissue. Furthermore, ABHD5 knock down data from mice show clear tissue specific difference by how ABHD5 acts as liver and fat storage tissue. Therefore, the different physiology in insects is accompanied by a different molecular mechanism that finally results in similar cellular changes in the absence of puml ${ }^{1}$.

In this context the importance of a proper peroxisomal lipid metabolism should be emphasized. Peroxisome biogenesis disorders (PBDs) are highly complex multi-organ dysfunction orders that exhibit a wide range of defects. The Zellweger syndrome is one of four groups of PBDs that is caused by mutations in the two peroxin (pex) genes PEX3 and PEX16. A Drosophila model for Zellweger disease was generated by the disruption of pex3 and pex16 gene (Nakayama et al., 2011). Various phenotypes could be observed in pex3 mutants such as larval lethality, shortened longevity, locomotion defects and abnormal lipid metabolism (Nakayama et al., 2011, Faust et al., 2014). Additionally, homozygous pex16 mutant male flies were sterile (Nakayama et al., 2011).

Whereas fecundity of male flies was not impaired (Rosenberg, 2012), pum/ ${ }^{1}$ flies shared other characteristics of Zellwegers disease. Longevity of pum/ ${ }^{1}$ flies was significantly decreased (Figure 16) and lipid metabolism was altered. Mutually, puml ${ }^{1}$ as well as pex $16^{1}$ and pex $10^{1}$ flies (Faust et al., 2014) accumulate VLCFAs. 
PBDs patients feature various neurological defects among them motor dysfunctions (Steinberg et al., 2006). Along with this pex $16^{1}$ flies showed locomotion deficits in climbing (Nakayama et al., 2011). Similarly, preliminary data indicate lower startle induced climbing activity in puml ${ }^{1}$ as well (Figure 38). A possible reason for neurological defects may be an impaired sphingolipid-production. Sphingolipids are crucial for a proper neuronal signal transmission and peroxisomes are the nascent site for this lipid. Thus, sphingolipid generation should be addressed in pum/ ${ }^{1}$ flies in the future as it might be impaired in puml $I^{1}$ flies. Complementary to this pum/ ${ }^{1}$ flies should be screened for other neurological disorders. By this pum/ ${ }^{1}$ would provide an additional suitable invertebrate system to study neurological defects associated with a disturbed lipid metabolism.
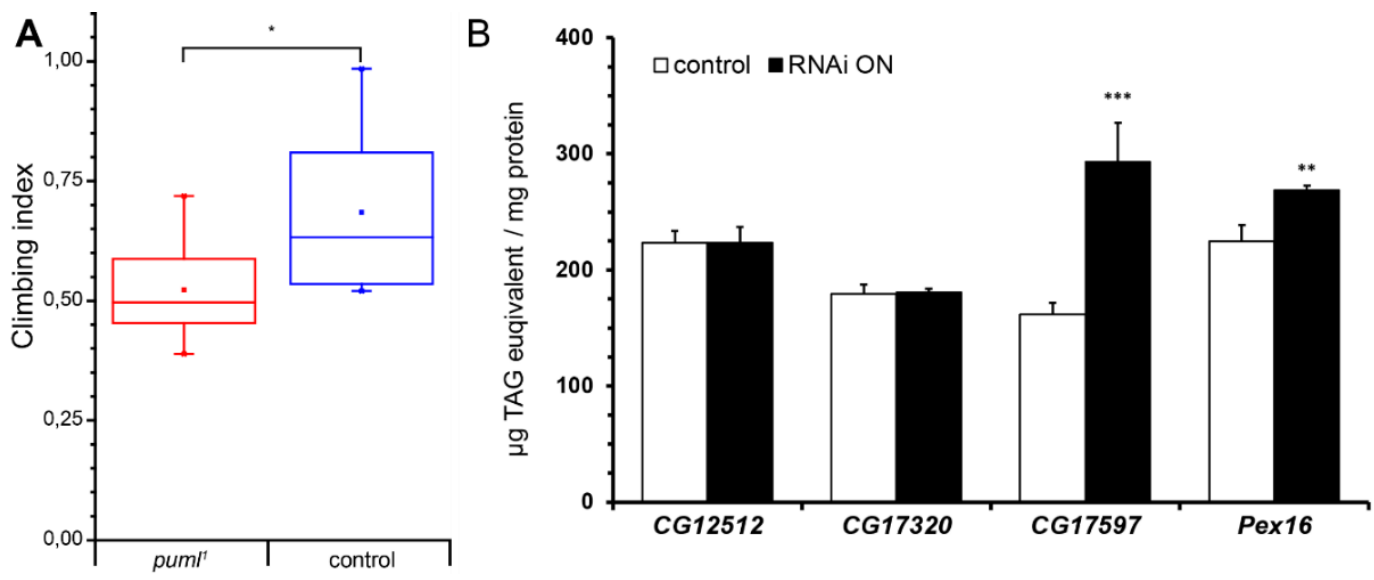

Figure 38 Preliminary results indicate that startle induced climbing activity is decreased in puml ${ }^{1}$ flies and a knock down of predicted peroxisomal $\beta$-oxidation genes can lead to increased body fat storage. A climbing index was calculated for puml $l^{1}$ and control flies. Startle induced climbing activity was significantly decreased in pum ${ }^{1}$ flies (A). A fat body specific (FB-SNS>GAL4) knock down of different candidates for peroxisome located $\beta$-oxidation and peroxisome biogenesis (Pex16) was performed. Pex16-RNAi as well as CG17597-RNAi (coding for protein with predicted $\beta$-ketothiolase activity) caused a significant increase in body fat storage of adult flies (B). The climbing index $(A)$ is shown as a Box plot from individual climbing indices from eight different fly cohorts (20 flies per cohort). Center lines show the median, box limits indicate $25^{\text {th }}$ and $75^{\text {th }}$ percentiles as determined by OriginPro software; whiskers extend 1.5 times the interquartile range from the $25^{\text {th }}$ and $75^{\text {th }}$ percentiles; $n \geq 136$ for each genotype (A). Averages of protein normalized TAG amounts \pm SEM are shown for the body fat measurements (Mann-Whitney test, ${ }^{* * *}=P<0.001 ;{ }^{*}=P<0.01$ ).

As shown in this work Puml resides on lipid droplets and peroxisomes (Figure 30) mutants that induce PBDs support the involvement of Puml in channelling lipids towards the peroxisomal pathway in a supportive manner. Indeed, preliminary data from RNAi mediated knock downs of selected genes predicted to be involved in peroxisomal $\beta$-oxidation (Faust et al., 2012) provided some evidence, that an 
impairment of this metabolic pathway can be already sufficient to increase body fat storage (Figure 38). So far only one candidate could be confirmed to actually change body fat storage, but candidates predicted by Faust and colleagues (Faust et al., 2012) were assigned based on similarity to known vertebrate genes. Therefore, redundancies are possible as well as different functions of the annotated candidate genes that are largely uncharacterized so far. However, the actual localization of these genes remain to be addressed as well in order to assign the effects of a knock down exclusively to peroxisomes.

Interestingly, peroxisome proliferator-activated receptor alpha (PPAR- $\alpha$ ) target gene expression was decreased in ATGL knock out mice (Haemmerle et al., 2011). PPAR- $\alpha$

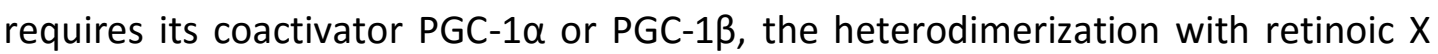
receptors and cognate lipid ligands for its function as a transcriptional activator. Transcription targets genes are important for fatty acid transport, oxidative phosphorylation, ketogenesis and gluconeogenesis (Lefevre et al., 2001, Sharma and Staels, 2007). Besides augmented adipose tissue mass ATGL deficient mice exhibit decreased expression of genes responsible for oxidative phosphorylation, ectopic lipid storage in multiple tissues and suffer from severe skeletal- and cardiomyopathies (Huijsman et al., 2009). These phenotypes can be retrieved in human patients of NLSD. It was therefore assumed that ATGL was needed to produce crucial mediators in order to generate lipid ligands that activate PPARs. Consistent with lowered PPAR- $\alpha$ activation in ATGL knock out mice, cardiac mitochondrial respiration was impaired. Triglyceride storage was elevated and glycogen was increased in cardiomyocytes. Additionally, although the overall structure of mitochondria was not affected and cristae appeared normal, mitochondria in ATGL knock out mice were significantly enlarged (Haemmerle et al., 2011). Interestingly, preliminary data shows also an increased size of mitochondria in malpighian tubules of $\mathrm{bmm}^{1}$ and puml $\mathrm{l}^{1}$ flies (Figure 39). 


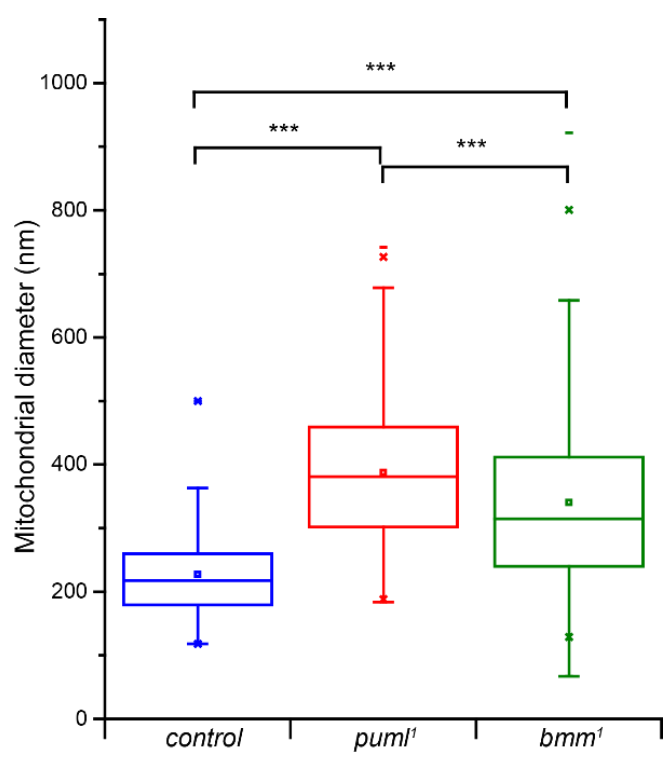

Figure 39 Preliminary data indicates that Mitochondria in Malpighian tubules from puml $\mathbf{l}^{1}$ and $^{b_{m} m^{1}}$ flies are enlarged. Box plot of mitochondrial diameter quantified in electron microscope pictures (Sample processing and image acquisition were performed by Dietmar Riedel) of Malpighian tubules. Center lines show the median, box limits indicate $25^{\text {th }}$ and $75^{\text {th }}$ percentiles as determined by OriginPro software; whiskers extend 1.5 times the interquartile range from the $25^{\text {th }}$ and $75^{\text {th }}$ percentiles (MannWhitney test; $n_{\text {mitochdria }}$ analysed per genotype: bmm $^{1}=291$, puml ${ }^{1}=123$ and control=96).

Drosophila hepatocyte nuclear factor 4 ( $\mathrm{Hnf} 4)$ acts ortholog to PPAR in mammals and regulates the expression of nuclear and mitochondrial genes needed for an efficient oxidative phosphorylation (Palanker et al., 2009, Barry and Thummel, 2016). Hnf4 mutant flies are hyperglycaemic, glucose intolerant, have impaired glucosestimulated insulin secretion and extremely short lived. Of course, metabolic defects can be observed already in Hnf4 deficient larvae (Palanker et al., 2009) the effects become highly pathological in adults (Barry and Thummel, 2016). Interestingly, expression of Hnf4 is vastly increased at the onset of adulthood (Barry and Thummel, 2016) that may explain the more pronounced metabolic defects and indicates the importance of Hnf4 for adult energy homeostasis. As the size of mitochondria is altered it might be possible that $b m m^{1}$ and pum/ ${ }^{1}$ flies produce less lipid mediators for a proper Hnf4 function leading to a reduced expression of oxidative phosphorylation genes. Whether $b m m^{1}$ and/or puml flies have reduced mitochondrial respiration needs to be investigates further. However, an overexpression of $\mathrm{bmm}$ in the fat body is known to protect flies from HFD induced ectopic lipid storage in cardiomyocytes (Birse et al., 2010). Therefore, a positive feedback loop for the lipid mobilization and energy production may be conserved in 
flies as well. In this model Bmm and/or Puml not only mobilize lipids but may provide additionally lipid mediators for Hnf4 activation that finally leads to improved oxidative capacity in the fly. As Hnf4 is highly expressed in the midgut, oenocytes, the fat body and in malpighian tubules (Gelbart and Emmert, 2013) this might be the reason why we can observe elevated body fat and ectopic lipid storage in malpighian tubules of $b m m^{1}$ and puml ${ }^{1}$.

Besides TAG species with even numbers of esterified fatty acids the pilot experiment measured also TAG species with odd C-atom numbers in the esterified fatty acids (Figure 32, Figure 33). These odd TAG species are actually common in Drosophila (personal communication from Vincenz Hofferek [MPImp-golm] and Dr. Ralf Pflanz[MPI-BPC]) but most lipidomics studies do not annotate these lipids. The origin of odd-numbered fatty sidechains needs to be clarified but it is very likely that they are synthesized from C3-bodies and might derive from bacteria and might be just ingested by the flies. Besides this, the odd TAG species may contain branched fatty acids from fatty acid-hydroxy fatty acids (FAFHAs), a recently identified class of lipids that can act as signaling molecules modulating insulin sensitivity of cells (Yore et al., 2014). So far, the existence of FAHFAs (with odd and even numbers of C-atoms) need to be confirmed in flies but from their general structure they might be also potential targets for Puml and conceivably this recently identified lipid signaling might be evolutionary conserved between invertebrates and mammals as well. Interestingly, peroxisomes are required for a processing of branched lipids (Vanhoe et al., 1993, Seedorf et al., 1994) indicating the importance of this organelle for lipid metabolism. In addition, FAHFAs would provide another connection between lipid metabolism and insulin signaling.

\subsection{A new insight in lipid storage control in Drosophila}

In flies, a complex regulatory network permanently monitors metabolic pathways and adapts the synthesis and mobilization of energy stores to the current metabolic state in order to maintain energy homeostasis. With their high volumetric energy density neutral lipids represent the major energy store in flies. At the same time these versatile molecules cannot only be used for oxidative phosphorylation (after $\beta$ - 
oxidation) but also serve as precursors for glyco- or phospholipids, are used for posttranslational protein modifications or even act directly as signaling molecules (Berg et al., 2007). As an imbalance in lipid generation, storage and turnover can have deleterious effects on the organismal health, high efforts have been taken to identify the underlying mechanisms and players of lipid metabolism. In this context Drosophila melanogaster provides a powerful model system for basic and applied research.

In this work I extended the commonly accepted model for lipid storage modulation in adult flies (Figure 37). The current model provides an explanation for the observed lipid storage phenotypes and carbon flux in pum $l^{1}$ flies.

According to the proposed model Puml would act on LDs by modulating lipogenesis and possible during lipolysis. Additionally, Puml would aid in the channelling of lipids to peroxisome and possibly may modulates maturation of late endosomes/lysosomes.

The important feature to perform these actions might be combinatorial. By modulating PA levels Puml would regulate AMPK and TOR signaling in flies. With a downregulation or absence of puml expression PA levels may locally increase and inactivate AMPK leading to decreased Bmm mediated lipolysis and increased lipogenesis due to increased insulin signaling in flies. However, during time of energy expenditure or nutrient deprivation this mechanism could be overwritten. Based on the assumption that PA derives mostly from DAG, that is generated continuously by basal TAG hydrolysis on lipid droplets and is normally re-esterified und basal conditions, starvation leads to post-translational modifications of various lipid storage modulators and changes their localization. By the activation and translocation of $D m H s l$ the DAG pool would be rapidly decreased and would diminish PA amounts. This would improve AMPK signaling and lower the insulin/TOR cascade leading to increased expression of lipolytic, peroxisomal and mitochondrial genes. At the same time AMPK activation would inhibit ACC leading to higher activity of CPT1 improving the lipid shuttling into mitochondria for oxidative phosphorylation. By this 
mechanism lipid utilization for energy production would not be impaired in pum ${ }^{1}$ and at the same time would explain why puml ${ }^{1}$ show increased lipid storage.

Of course, the very important piece in this puzzle (PA amounts) needs to be investigated. Probably an extended lipidomics approach is needed to detect possible changes as the concentration differences are more likely expected in a very low molar range than in changed amounts of $\mu \mathrm{g}$ that would be detectable on a TLC using whole fly extracts. Indeed, ABHD5 knock out mice actually exhibit increased PA levels indicating that, besides its ATGL stimulating character, ABDH5 may possesses a so far unknown function for regulating PA and may the current working model can be applied to humans as well. Thus, the ATGL activating function of ABHD5 embodies an acquired evolutionary adaption to rapidly boost TAG hydrolysis, while at the same time providing a two factor control system to tightly adapt neutral storage lipid mobilization.

The current working model provides an idea why $A K H R^{1}$ flies mobilized lipids during starvation (Grönke et al., 2007). Of course the primary signaling cascade induced by AKH binding would be impaired. At the time the insulin signaling would decrease leading to less inhibition of dFoxo and subsequently increased expression of lipolytic genes like pudgy or bmm (Xu et al., 2012). With a positive feedback loop by Hnf4 lipolysis would be enhanced without AKHR signaling. Due to the involvement of insulin signaling, expressional changes and protein translation this would provide cause for the delay (Grönke et al., 2007) in enhanced lipolytic activity in $A K H R^{1}$ flies and why lipolysis does not occur in $A K H R^{1} \mathrm{bmm}^{1}$ double mutant flies. With a possible activation of Bmm by phosphorylation the increased lipolytic activity would majorly be driven by the higher amounts of $\mathrm{Bmm}$ in $A K H R^{1}$ flies. The basal expression in normal flies would provide a basic pool of Bmm protein that is responsive for fast adaptions to lipid mobilization needs via AKHR signaling before transcriptional and translational processes enhances lipolysis and energy production. However, $\mathrm{bmm}$ deficient flies are still capable to mobilize lipids. Although, the lipolytic activity is less enhanced in $\mathrm{bmm}^{1}$ flies it is dependent on Plin1 (Grönke et al., 2007) indicating that maybe $D m H s$ or so far unknown lipases contribute to this activity. Yet, changes in 
mitochondria size of $b m m^{1}$ flies implicate that respiratory capacity might be lower due to impaired Hnf4 signaling lowering the oxidative capacity of these flies.

Taken together, to consolidate the current working model additional should be performed. A critical factor are the PA levels that represent the signaling molecule that links lipid- and carbohydrate metabolism. It should be addressed with high priority in puml ${ }^{1}$ flies. Also, the proposed inactivation of AMPK but stabilized Akt and TOR should be shown in pum ${ }^{1}$ flies. This could be done by immunohistochemistry of these proteins directly or quantification of gene expression from transcriptional targets of the respective pathways. Postulated high amounts of Akt in pum/ ${ }^{1}$ flies should provide a higher phosphorylation (and thereby inactivation) of GSK3 (shaggy) and subsequently Glycogensynthase (explains low Glycogen storage due to decreased synthesis), dFoxo and Dp53. The stabilized Tor as well as increased phosphorylation of S6K and PI3K would provide an evidence for the increased insulin sensitivity of pum ${ }^{1}$ flies. In this context it would be also interesting how blood sugar levels in puml ${ }^{1}$ flies are.

Consistently with the increased lipogenesis detected in puml ${ }^{1}$, it should be checked if genes like fas and $m d y$ are upregulated and pudgy and $b m m$ are downregulated under feeding conditions. As lipid mobilization in puml $^{1}$ flies is elevated under starvation it should be checked if glycolytic genes like pyruvate kinase or hexokinase $c$ are downregulated. Whether basal lipolysis is increased as well has not been addressed in pum ${ }^{1}$ flies so far. Hence, it would provide an explanation for the observed higher labelling of phospholipids in the radioactive glucose feeding assay as a results of increased PL synthesis and turnover.

As pum/ was upregulated in mio flies on a high sugar diet it would be interesting to see if puml is a direct target of mio. A mio-gof should suppress puml expression and should lead to increased lipid storage accompanied with lower glycogen storage.

A last point should be the analysis of the oxidative capacity of $\mathrm{bmm}^{1}$ and pum/ ${ }^{1}$ flies. The enlarged mitochondria provide some evidence for an impairment but this should correlate with decreased expression of mitochondrial genes like cytochrome coxidase (COX), pyruvate dehydrogenase (PDH) or succinate dehydrogenase (SDH). 
With the possible participation of $\mathrm{bmm}$ and puml in Hnf4 signaling, additional adult tissues in adult flies of the respective mutants should be screen for elevated lipid storage e.g. oenocytes or cardiac muscles.

In conclusion puml modulates carbon flux in Drosophila melanogaster but is not crucial for the survival. Certain characteristics of the proposed mechanism by how pum/ mediates the metabolic regulation might be conserved between mammalian ABHD5 (lipid over-storage, ectopic lipid storage). However, pum/ seems to perform additional functions in flies that are not shared with ABHD5 (no activation of Bmm by Puml) but partially with its mammalian paralog ABHD4 (e.g. phospholipase activity). Combined, the identified set of characteristics of Puml may represent the ancestral function of this protein family. Evolutionary adaptions and gene duplication generated the two paralogs ABHD4 and ABHD5 that retained parts of their original functions but might be regulated differently nowadays (changed intracellular localization or different tissue expression profiles) or acquired new functions (ATGL activation by $\mathrm{ABHD5}$ ) due to changed organismal demands on energy homeostasis. Therefore, pum ${ }^{1}$ flies may serve as an animal model for neutral lipid storage disease to find therapeutics to treat this human disease. 


\section{Supplement 1}

\subsection{Characterization of DmHsl (CG11055)}

Lipid storage and mobilization is a well-orchestrated process that can be performed by most if not all cells. In mammals, a specific set of proteins has been identified that play a key role in the mobilization of storage lipids. Among them are: Adipocyte triglyceride lipase (ATGL) (Zimmermann et al., 2004) with its activator ABHD5/CGI-58 (Lass et al., 2006), hormone-sensitive lipase (HSL) (Haemmerle et al., 2002a) and Perilipins (Greenberg et al., 1991). For mammalian ATGL and Perilipin1 homologues can be found in Drosophila melanogaster namely Brummer lipase (Grönke et al., 2005) and Perilipin1/Lsd-1 (Beller et al., 2010).

As presented in the current work a sequence-related protein can be found for ABHD5 in flies, that has been named Pummelig (Puml/CG1882), but the mechanism by how Puml is modulating lipid storage seems not be evolutionary conserved.

Mammalian hormone-sensitive lipase has been studied for over half a century now and was long considered to be the sole key lipase for induced lipolysis in adipose tissue. Only a decade ago ATGL was identified as the important lipase in the first step of triacylglyceride (TAG) mobilization (Zimmermann et al., 2004) that also underlies an additional level of regulation. For an optimal TAG hydrolase activity, ATGL relies on its activator ABHD5 (Lass et al., 2006, Granneman et al., 2007) that competes with the ATGL inhibitor GOS2 (Cerk et al., 2014). Additionally, ABHD5 serves as a platform to recruit fatty acid binding protein (FABP) creating a sort of lipid mobilization complex. Further on ABHD5 is localized on lipid droplets under basal conditions and requires Perilipin1 as a binding partner (Granneman et al., 2007, Granneman et al., 2009).

Upon a lipolytic stimulus, e.g. by $\beta$-adrenergic signalling, PKA phosphorylates PLIN1 (pPLIN1) (Tansey et al., 2001, Tansey et al., 2004) and HSL (pHSL) (Huttunen et al., 1970). This leads to the release of ABHD5 from pPLIN1 that now can interact with the pHSL which in turn translocates from the cytoplasm onto the lipid droplet (Sztalryd et al., 2003). The released ABHD5 competes with the ATGL inhibitor GOS2. With the protein interaction of ATGL-ABHD5 (Lass et al., 2006, Granneman et al., 2007) this 
complex localizes onto LD surface again and lipolysis starts. In a three-step process the first fatty acid (FA) from TAGs is cleaved off by ATGL. Subsequently, the DAG is hydrolysed by HSL and in the last MAG is cleaved by MAGL into FA and the glycerol backbone.

In Drosophila two perilipins can be found that have distinct roles in lipid metabolism. A study of the lipid droplet proteome revealed that LDs from Dmplin1, Dmplin2 single- and Dmplin1,Dmplin2 double-mutants are populated differently by proteins (Sahu-Osen, 2015) indicating the modulating role of perilipins by controlling access of proteins to LDs.

Dmplin1 mutant flies have increased lipid storage with a giant lipid droplet phenotype, are hyperphagic but lipolysis in general is not impaired (Beller et al., 2010). Body fat storage of Dmplin2 mutants, on the other hand, is decreased and overexpression leads to obesity (Grönke et al., 2003).

As mentioned before a complete breakdown of glycerolipids into fatty acids and the glycerol backbone is a three step process. Brummer has been described as an important TAG lipase (Grönke et al., 2005). bmm mutants have increased body fat storage, increased starvation resistance (Grönke et al., 2005). Whereas, a single knockout of $\mathrm{bmm}$ is still capable to mobilize storage lipids a double knockout of $\mathrm{bmm}$ and Dmplin1 cannot mobilize storage lipids at all (Beller et al., 2010). In mammals HSL had been identified as the main DAG lipase in adipose tissue (Fredrikson et al., 1986, Haemmerle et al., 2002a).

In order to see if the core lipid mobilization module in mammals is evolutionary conserved in the fly a search for a HSL homolog was performed and DmHsI (CG11055) was identified as the sole member of the HSL-family in Drosophila. To study the function of $\mathrm{DmHsl}$ a knockout mutant was generated and initially characterized by Sebastian Grönke (Grönke, 2005). Comparable to $\mathrm{HSL}^{-/}$knockout mice (Haemmerle et al., 2002a) total body fat was not increased in $\mathrm{DmHs}^{1}$ flies and starvation resistance was not changed compared to control flies (Grönke, 2005). DmHsl is expressed during all developmental stages of Drosophila melanogaster with a strong enrichment in early embryonic stages (0-3h) indicating a maternal contribution 
(Grönke, 2005, Bi et al., 2012, Gelbart and Emmert, 2013). In larvae, comparable to $\mathrm{bmm}, \mathrm{DmHsl}$ expression is increased during starvation (Bi et al., 2012). A mutant independently generated and characterized by (Bi et al., 2012) exhibited increased lipid storage (+30\%) and lipid mobilization deficits during starvation in larvae.

An important characteristic of mammalian HSL function is its translocation from the cytoplasm to LDs that requires pPLIN1 on LDs (Sztalryd et al., 2003). However, known phosphorylation sites in mammalian HSL are only poorly conserved in the fly (Grönke, 2005). Several studies identified $D m H s l$ as a lipid droplet resident (Cermelli et al., 2006, Krahmer et al., 2013). A in vivo approach, using a fat body specific overexpression of DmHsl::eGFP showed in larvae that DmHsl::eGFP was majorly localized in the cytoplasm under fed conditions (Bi et al., 2012). In addition, abundance of DmHsl::eGFP on LDs was higher during nutrient deprivation (Bi et al., 2012). While $D m H s l:: e G F P$ still showed a LD localization in plin $2^{1}$ larvae it was nearly absent on LDs in plin $1^{1}$ larvae indicating a conserved role of DmPlin1 in sequestering DmHsl::eGFP onto the LD surface (Bi et al., 2012).

Studies on $\mathrm{DmHsl}$ concentrated mainly on larval stages and the data indicate a conserved function to mammalian HSL (Grönke, 2005, Bi et al., 2012). Therefore, $\mathrm{DmHs} /$ was analysed further to characterize its function in adult flies and identify a possible biological phenotype in $\mathrm{DmHs}{ }^{1}$ flies.

\subsection{Body fat storage is not altered in $\mathrm{DmHs} \mathbf{I}^{1}$ flies}

The $D m H s l^{1}$ fly stock was generated by an imprecise P-element excision and has been initially characterized by Sebastian Grönke in 2005. Body fat measurements revealed no changes in $D m H s I^{1}$ flies in comparison to a generated control and average mean survival times under starvation did not exceed controls (Grönke, 2005).

In order to avoid the detection of false-positive phenotypes deriving from inbreeding effects of the $D m H_{s} I^{1}$ strain and having a proper genetically matched control the $\left.D \mathrm{mHs}\right|^{1}$ strain was backcrossed for ten generations into a $w^{1118}$ background and a homozygous $\left.\mathrm{DmHs}\right|^{1}$ stock was established again. 


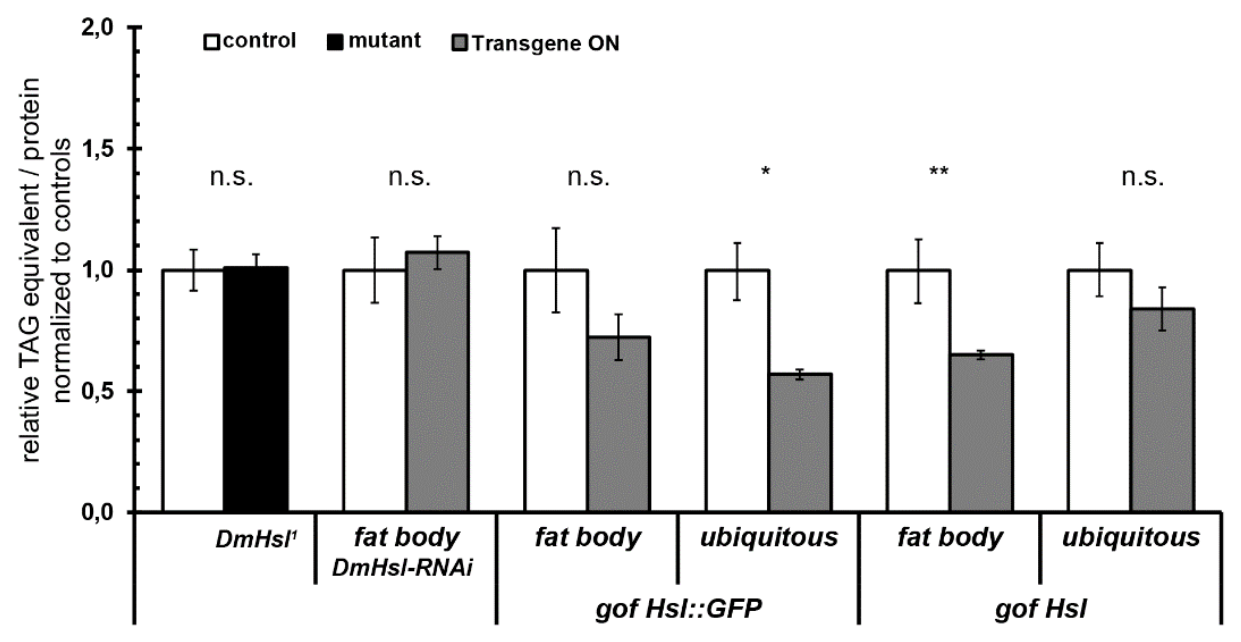

Figure 40 Body fat storage is unchanged in $\mathrm{DmHs}^{1}$ flies. An DmHsl-RNAi in the fat body (FBSNS>GAL4) showed the same phenotype. A fat-body targeted (FB-SNS>GAL4) or ubiquitous (Act5c>GAL4) overexpression of GFP-tagged and non-tagged DmHsl lead to reduced body fat storage but the effect was highly variable. Plotted are means of relative TAG equivalents/protein normalized to average TAG equivalents/protein values of the corresponding controls \pm SEM; Mann-Whitney test; $\left.*^{*}=P<0.01 ; *=P<0.05\right)$.

A reanalysis of body fat content in backcrossed $D m H s l^{1}$ flies verified the results from

S. Grönke. The overexpression of $\mathrm{DmHsl}$ and DmHsl::GFP always showed a trend towards lower body fat but the effect was highly variable (Figure 40).

\subsection{Diacylglycerols are not elevated in $\left.\mathrm{DmHs}\right|^{1}$ flies}

Consistent with data from mice total amounts of glycerolipids are unchanged in adult $D m H s{ }^{1}$ flies (Figure 40). However, in accordance with the high Diacylglycerolhydrolase activity of MmHSL (Fredrikson et al., 1986), DAGs levels are elevated in HSL knock out mice (Haemmerle et al., 2002a). Therefore, a TLC analysis was performed in order to separate and quantify the different neutral lipid classes in $D m H s I^{1}$ flies. In comparison to control flies no neutral lipid class (TAG, DAG, MAG, FA) was changed in lipid extractions from total $\mathrm{DmHs}{ }^{1}$ compared to control flies (Figure 41). Lipids are transported mainly as DAGs in the hemolymph of flies (FernandoWarnakulasuriya and Wells, 1988, Pennington and Wells, 2002). Therefore, it might be possible that DAG accumulations only can be observed in lipid degrading tissues and overall relative amounts might be low in total fly lipid extracts. In order to answer this question, TLC analyses were performed from lipid extractions deriving from 
muscle enriched samples (Thorax) and lipid storing tissues like fat body and intestine (Abdomen sample).

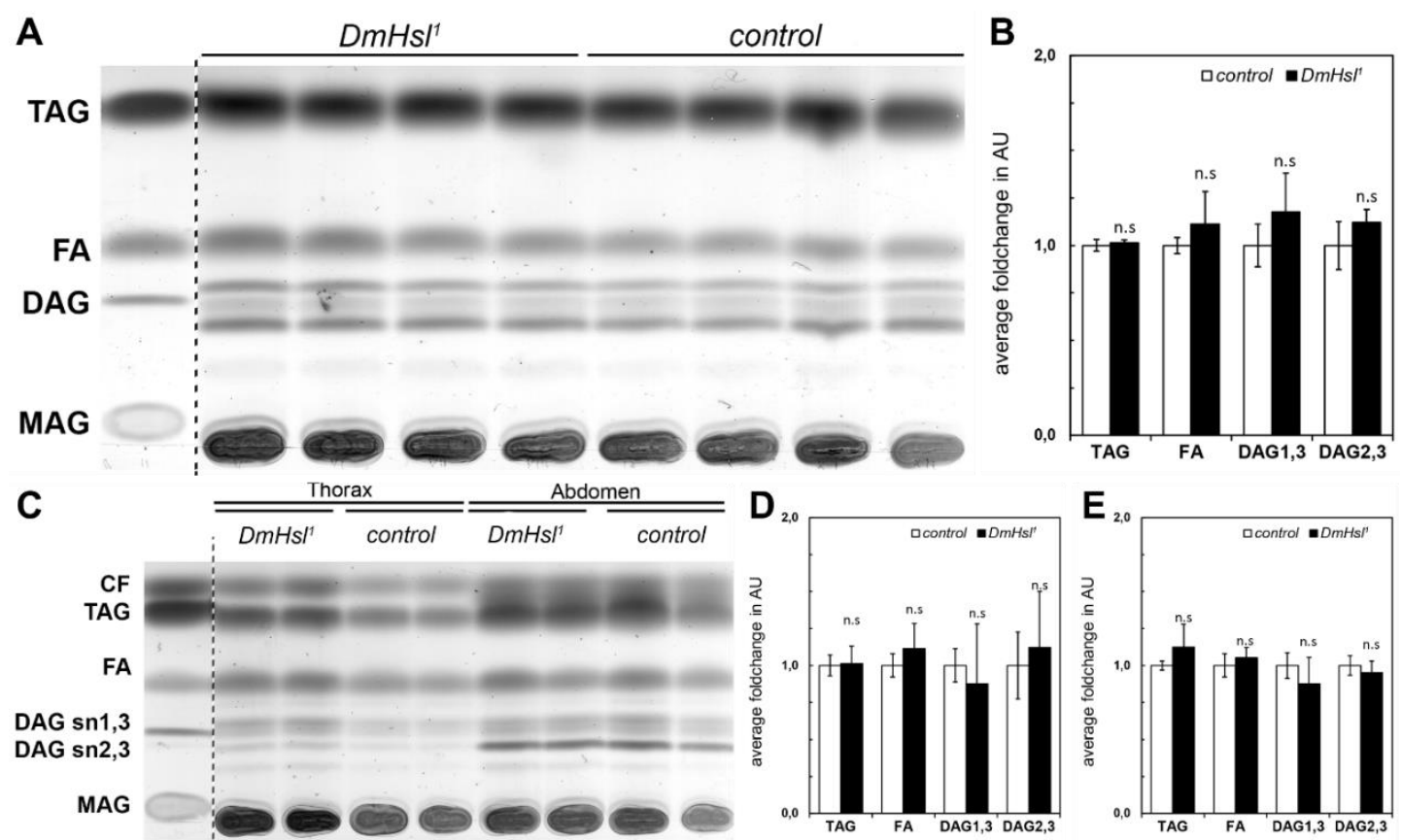

Figure 41 Neutral lipid classes are unchanged in $\mathrm{DmHsl}^{1}$ flies. (A, B) TLC analysis from $\mathrm{DmHs}{ }^{1}$ and control flies. (C, D, E) TLC analysis of $\mathrm{DmHs}^{1}$ and controls using lipid extractions from thorax (muscle tissue) and abdomen (enriched in fat body and intestinal tissue). (B, $D, E)$ Plotted are the means of average fold changes in Arbitrary units (AU; measured densiometrically using ImageJ $v 1.49 m$ ) for the annotated lipid classes compared to control flies \pm SEM; Student's $t$-test. Dotted lines $(A, C)$ mark a break due to a removal of a lane on the TLC plate. Cholesterol formate (CF) was used in C as an internal control for extraction yield. Measured AUs in D,E and were normalized for CF and then compared between the samples.

Also in the muscle enriched tissue samples no differences could be detected between $D m H s{ }^{1}$ flies and controls. Differences could be seen between the various tissue samples as DAG species appeared much more prominent in abdomen sample in general (Figure 41).

Taken together, besides an identical substrate spectrum of DmHsl compared to MmHSL, DAGs are not elevated in $\mathrm{DmHs}^{1}$ indicating a redundant mechanism of DAG hydrolysis in Drosophila melanogaster.

\subsection{DmHsl::EGFP abundance on LDs is higher during starvation in larvae and adults}

An important characteristic of $\mathrm{MmHSL}$ is its translocation from the cytoplasm onto lipid droplets upon phosphorylation by PKA and the interaction of phosphorylated 
PLIN1 in adipose tissue (Sztalryd and Kraemer, 1994, Lass et al., 2006). Overexpressed $D m H$ sl::EGFP shows a comparable behaviour in larval fat body cells and is more abundant on LDs during starvation (Bi et al., 2012). Bi et al. (Bi et al., 2012) also postulated that DmPlin1 is needed for a proper localization of DmHsl::GFP on LDs during starvation in larvae. As the studies on $\mathrm{DmHs}$ l concentrated on the larval stage I was interested if localization behaviour is the same in adult flies. Comparable to larvae (Figure 42) higher abundance of DmHsl::GFP (expressed in the fat body tissue [FB-SNS>GAL4]) on LDs could be observed in adults as well (Figure 43). Whereas $D m H s \mid:: G F P$ was omnipresent on small lipid droplets $(<8 \mu \mathrm{m})$ the abundance was most strikingly increased on large LDs $(>10 \mathrm{~m}=\mu \mathrm{m})$ during nutrient deprivation (Figure 45).

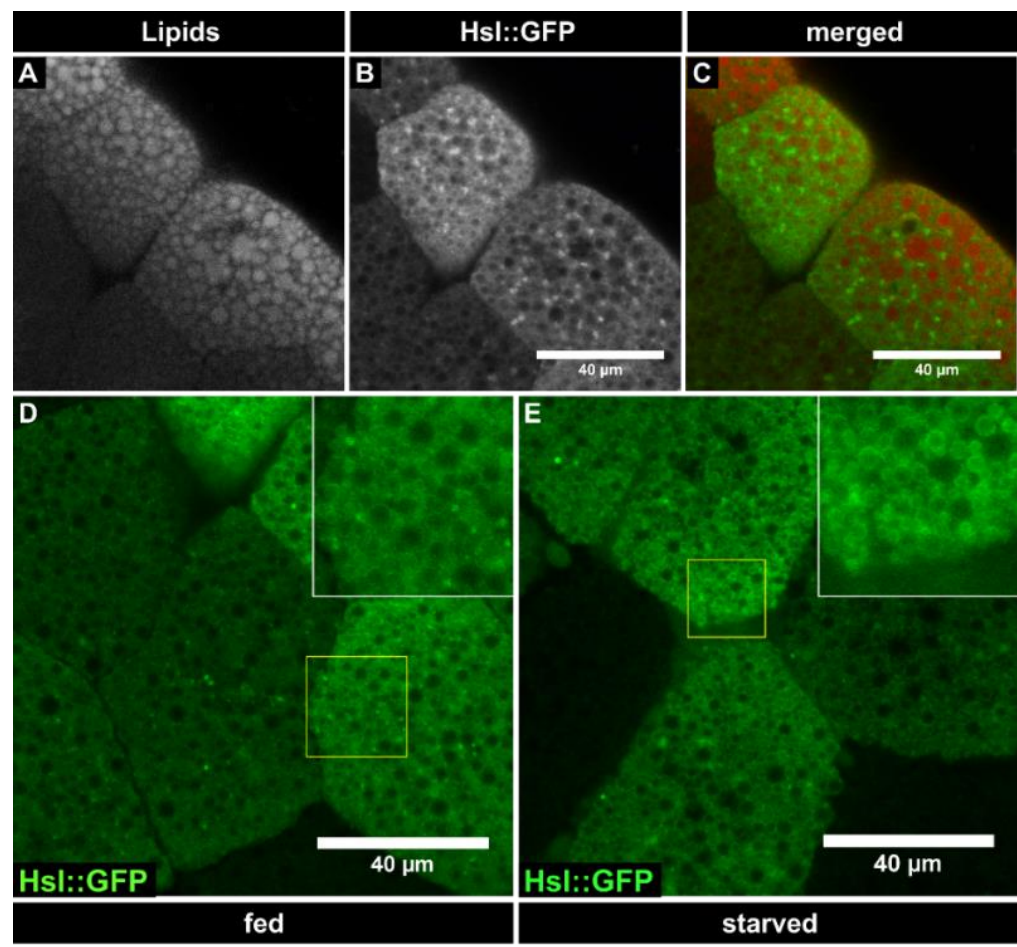

Figure 42 Hsl::GFP localizes on lipid droplets in larval fat body. Upper row shows lipid storage in fed animals (Lipids were stained by LipidTOX) and LD localization of fat body expressed (FB-SNS>GAL4) $H s l:: G F P$ (ring-like pattern in D). Ring-like localization on LDs become more evident (E) during starvation (early L3 larvae were starved for 6h). 

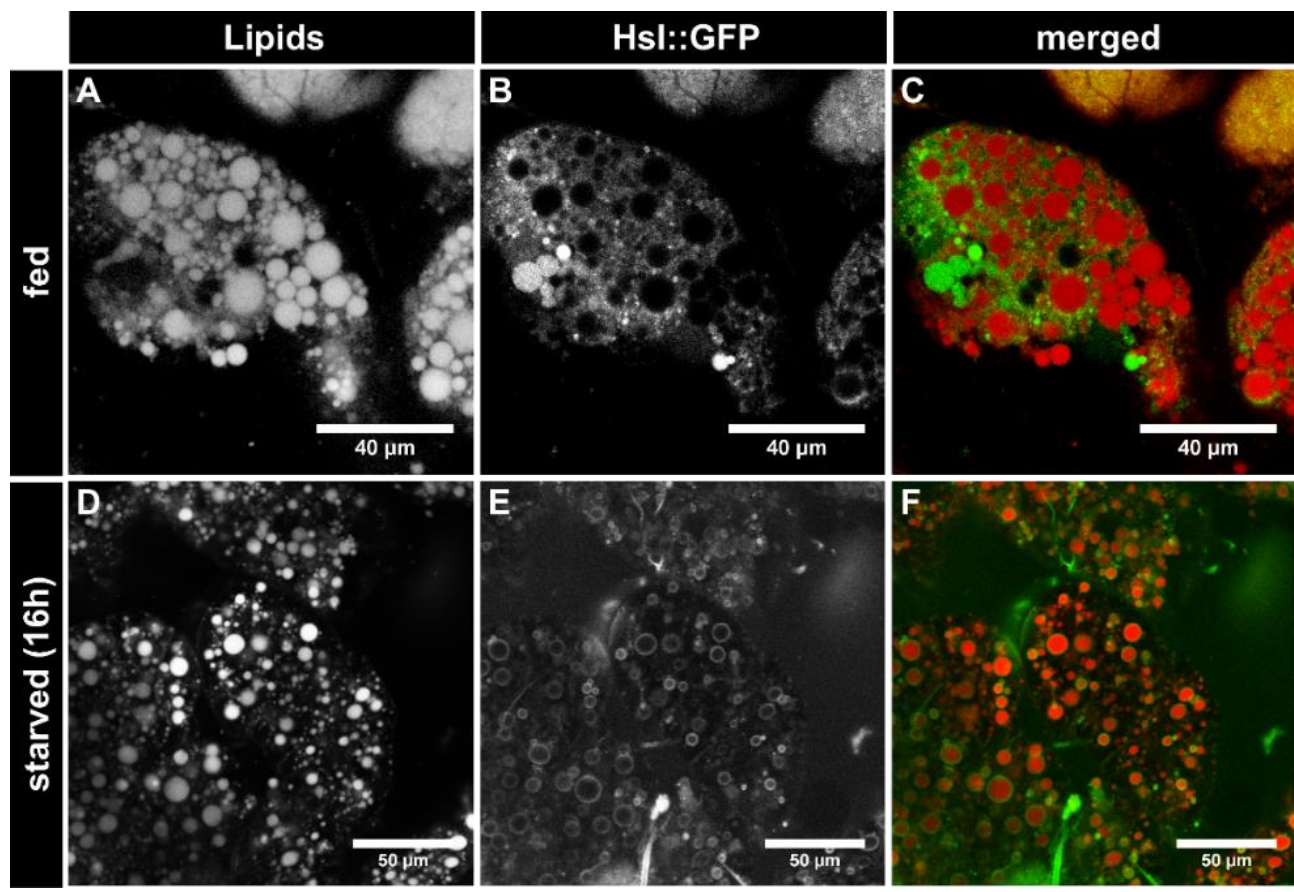

Figure 43 Hsl::GFP localization on lipid droplets also occurs in adult fat body tissue. Ring-like localization of fat body (FB-SNS>GAL4) expressed DmHsl:GFP on LDs is clearly visible under starvation. Additionally, Hsl::GFP signal appears to be more prominent on large LDs during starvation whereas it is mostly found on small LDs during feeding periods.

\subsection{Lipid mobilization in $D m H s l^{1}$ flies is not impaired}

The independently generated $\mathrm{DmHs}^{624}$ mutant (Bi et al., 2012) showed impaired lipid mobilization in larvae. With a comparable localization pattern of DmHsl::GFP under fed and starvation conditions a similar behaviour was expected in $\mathrm{DmHs}^{1}$ adult flies. For this, starved flies were starved and body fat was measured by CCA assay (Figure 44). However, $D m H s l^{1}$ flies could mobilize their lipids assuming a possible redundancy of $\mathrm{DmH}$ s/ function.

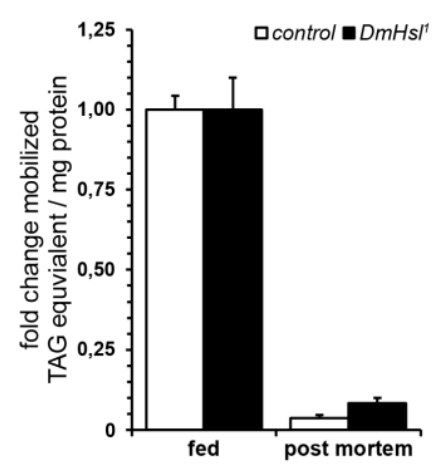

Figure $44 \mathrm{DmHs}^{1}$ flies can mobilize lipids. Average fold change of TAG equivalents / $\mathrm{mg}$ protein in fed and starved control and $\mathrm{DmHs}^{1}$ flies \pm SEM. Student 's test revealed no significant differences between the two genotypes. 
5.6DmPlin1 is crucial for localization of $D m H s \mid:: G F P$ on large LDs $(>10 \mu m)$ but not small

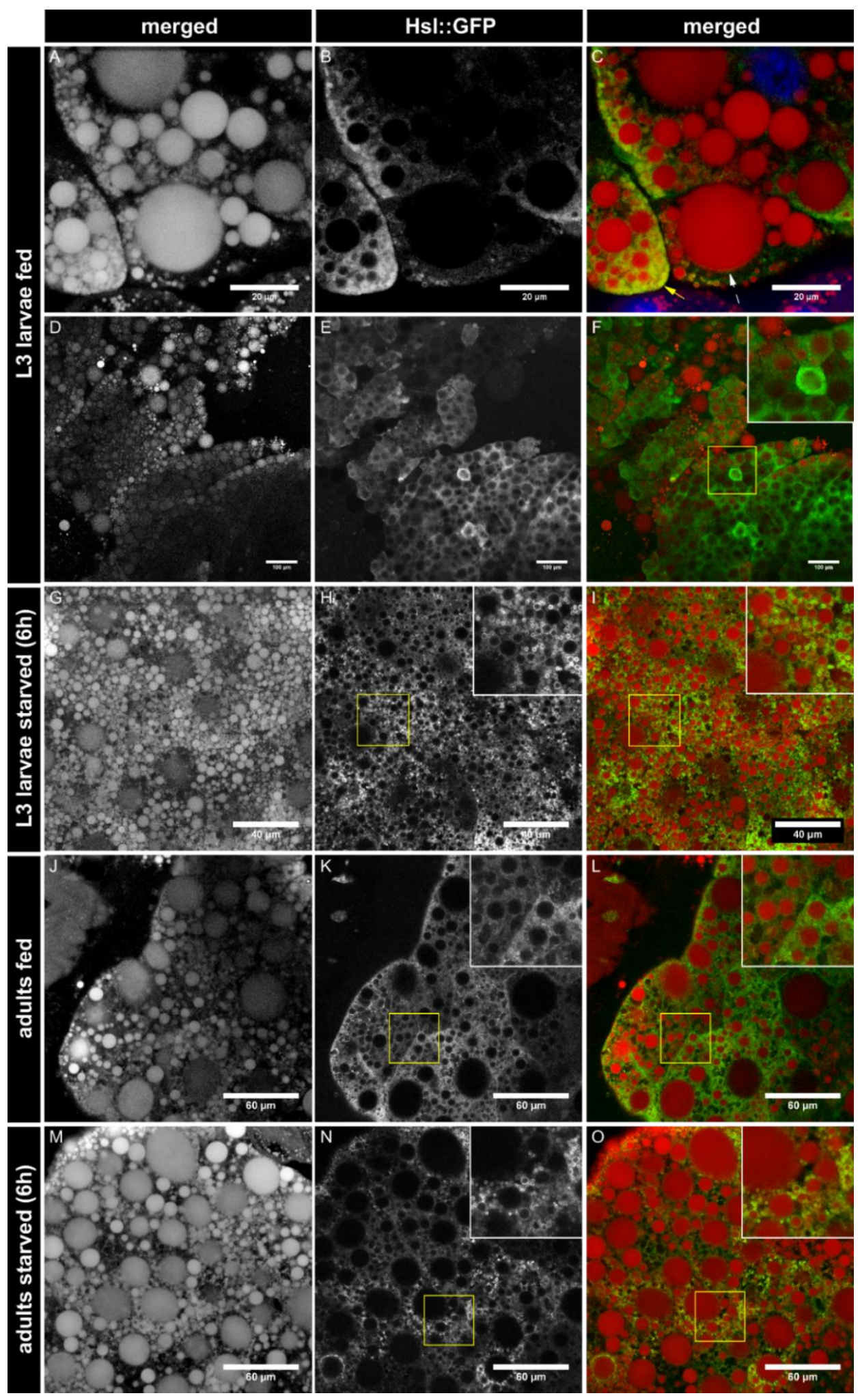

Figure 45 Hsl::GFP expressed in plin1 ${ }^{1}$ larvae and flies localizes on lipid droplets under fed and fasting conditions in larvae (A-I) and adults (J-O). Note, that giant lipid droplet phenotype is not changed $(F$, $J, M)$ drastically upon Hsl::GFP expression (FB-SNS>GAL4). Even during fasting conditions bigger lipid droplets $(>10 \mu \mathrm{m})$ seem to be spared from Hsl::GFP localization $(I, O)$. 
In order to see if the HSL sequestering role of PLIN1 is conserved in flies DmHsl::GFP was expressed in the fat body (FB-SNS>GAL4) in plin $1^{1}$ flies. Comparable to data from Bi et al. (2012) DmHsl::GFP could be found on LDs in general in larvae (Figure 45). The same was the case in the adult fat body (Figure 45). In plin $1^{1}$ larvae as well as in adult plin1 $1^{1}$ flies large LDs $(>10 \mu \mathrm{m})$ were spared from $D m H s \mid:: G F P$ signal. The large LD phenotype of plin $1^{1}$ was persistent in larvae and adults expressing $D m H s l:: G F P$. Whether the avoidance of $D m H s \mid:: G F P$ populating large LDs was due to the lack of its interaction partner PLIN1 or an indirect effect like the size and therefore the curvature of the LD itself remains to be answered.

\subsection{Fecundity in $\left.\mathrm{DmHs}\right|^{1}$ flies is not impaired}

$D m H s \mid:: G F P$ abundance on LDs is increased under starvation. Also, $\mathrm{DmHsl}$ has a comparable substrate spectrum to $\mathrm{MmHSL}$ (personal communication by Dr. C. Heier) but no increase in DAGs in DmHsl deficient flies. It was therefore assumed that $\mathrm{DmHsl}$ function is not exclusively limited to hydrolyse primarily DAGs in storage lipid mobilization. Expression data (Gelbart and Emmert, 2013) and in situ hybridisations (Bi et al., 2012) indicated a strong maternal contribution of DmHsl-RNA in embryos. Therefore, fecundity of $\mathrm{DmHs}{ }^{1}$ was analysed in order to address a possible function of $\mathrm{DmHsl}$ during embryogenesis indicating an important function of $\mathrm{DmHsl}$ during embryogenesis.
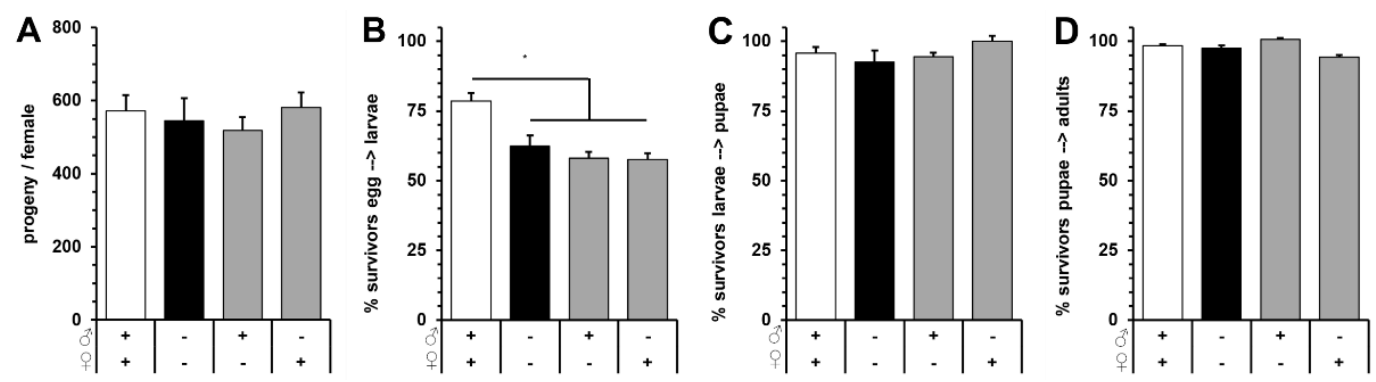

Figure 46 Fecundity is not impaired in $\mathrm{DmHsl}^{1}$ flies. $\mathrm{DmHsl}^{+}(+)$and $\mathrm{DmHs}^{1}$ (-) flies were used. Homozygous stocks were compared to crosses of female $\mathrm{DmHsl}^{1}$ and males from controls and vice versa to address a possible gender dependent phenotype of DmHsl deficiency. Total numbers of viable progeny did not vary significantly in the tested conditions (A). Lower survial rate of egg $\rightarrow$ larvae were compensated by higher egg deposition (B). Survival rates of larval stages and during metamorphosis are nearly $100 \%$ for all tested conditions (SEM, student test, ${ }^{*}=P<0.05, n>550$ / combination).

Total numbers of viable progeny were comparable between crosses of $\mathrm{DmHs}^{1}$, controls and heterogenic combinations crossing $\mathrm{DmHs}{ }^{1}$ virgins with male control flies 
and vice versa (Figure 46). Survival from egg to larval stages were relatively low but similar between the tested conditions. Only control crosses appeared to have higher survival rates. As total numbers of progeny per female were equal the disadvantage of the lower survival rate was compensated by higher egg deposition rates. Survival rates for the later development were at nearly $100 \%$ in all tested conditions. A significant effect on fecundity in $\mathrm{DmHsl}$ deficient flies could not be detected. Of course an involvement of $\mathrm{DmHsl}$ cannot be excluded due to possible redundancies for its function.

\subsection{Discussion}

Taken together total body fat storage is not changed in $\mathrm{DmHs}{ }^{1}$ flies compared to control flies. A detailed analysis of different neutral lipid species by thin layer chromatography and lipidomics revealed no increase in total DAG species as observed in $\mathrm{HSL}^{-/}$mice (Haemmerle et al., 2002a). However, the substrate spectra of DmHsl and mouse HSL in in vitro assays are identical (personal communication from Dr. Christoph Heier and Dr. Robert Zimmermann [University Graz]) covering hydrolytic activities on: TAGs, DAGs (Fredrikson et al., 1986) (highest activity), MAGs (Fredrikson et al., 1981) and Cholesterol esters (Contreras et al., 1998). This would argue in favor of an evolutionary conserved function in $\mathrm{DmHs}$ in flies. However, fly physiology differs from mammals as the main transport form of lipids in Drosophila are DAGs bound to lipoproteins (Palm et al., 2012). Therefore, it might be possible that there is a general redundancy of direct DAG lipolysis or a more flexible metabolism that allows a different processing of DAGs e.g conversion into a phospholipid and subsequent hydrolysis by specific phospholipases.

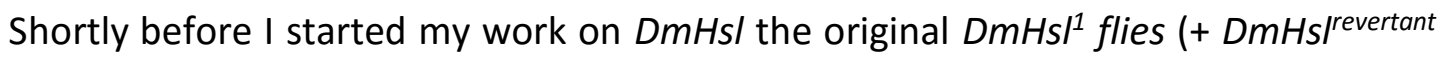
and Act5c>GAL4 UAS-DmHsl) were sent (prepared by Iris Bickmeyer and Dr. Ronald Kühnlein) for a lipidomics analysis performed by the Lipidomics facility from Medical University Graz (used were 2x50 six-day-old flies, from seeding 150 embryos / midsize vial; from two independent density seedings). The analysis from the annotated lipid data revealed a significantly higher total TAG storage of control flies ( $\mathrm{DmHs}$ /revertant) compared to the $\mathrm{DmHs}^{1}$ and a ubiquitous (Act5c>GAL4) overexpression of $\mathrm{Hsl}$ (Figure 
47). Total amounts of DAGs per fly were also highest in control flies and no significant differences could be observed between Hsl overexpressing of deficient flies (Figure 47). Nevertheless, the $\%$ of DAGs from total TAGs indicated a significant increase in $\%$ of DAGs of total lipids in $\left.D m H_{s}\right|^{1}$ and gof-DmHsl flies (Figure 47). No differences could be detected between the mutant and overexpression flies indicating that there is no significant increase DAG species in $\left.D m H s\right|^{1}$ flies. The observed differences are rather a results of a sub-optimal matched control for the $\left.D m H_{s}\right|^{1}$ stock as TAG amounts in backcrossed flies did not differ between $\mathrm{DmHs}{ }^{1}$ and controls (Figure 47). A TLC analysis (performed by Iris Bickmeyer) of flies used for the lipidomics analysis revealed differences in TAG storage but not in DAGs (data not shown).
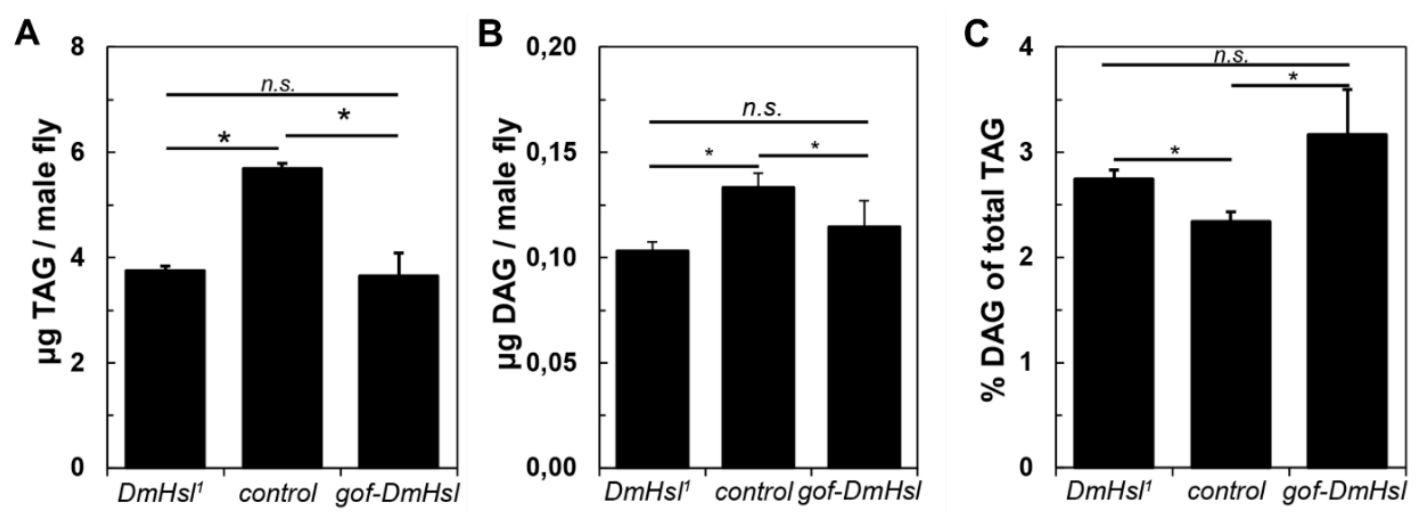

Figure 47 Preliminary results indicate that $\mathrm{DmHsl}^{1}$ flies do not accumulate diacylglycerol. A lipidomic analysis performed by the Lipidomics facility of the Medical University Graz using non-backcrossed $D m H s{ }^{1}$ flies (6d males, prepared by Iris Bickmeyer and Dr. Ronald Kühnlein) showed a small but significant increase in \%DAGs from total detected TAGs (C). Absolute amounts of TAGs and DAGs were significantly higher in control flies compared to $\mathrm{DmHs}^{1}$ but flies ubiquitously over expressing DmHsl (Act5c>GAL4) showed comparable total amounts. A strong accumulation of DAGs cannot be seen (consistent with TLC data). Plotted are the means of average lipid amounts of all detected TAG and DAG species detected per male flies $(A, B)$ and calculated \% of DAGs (C) of total TAGs ( \pm SEM; Student's t-test, $\left.{ }^{*}=P<0.05\right)$.

As fed flies were used for the TLC experiment as well as for the Lipidomics analysis (non-backcrossed $\mathrm{DmHs}^{1}$ stock was analyzed) a possible effect of DAG accumulations might be too mild in order to be detected. On the other hand, lipid mobilization in $\left.D m H s\right|^{1}$ flies was indifferent from control flies. The TLC analysis from abdomen and thorax samples exhibited a different lipid species profile with a strong enrichment in a lipid with a comparable running behavior as DAGs on the TLC plate. Though a difference between $\mathrm{DmHs}{ }^{1}$ and control flies could not be detected. Conversely, an 
overexpression of $\mathrm{DmHsl}::$ egfp exhibits a trend towards decreased body fat indicating a involvement in lipolysis of $\mathrm{DmHsl}$.

Comparable to studies in larvae (Bi et al., 2012) overexpressed DmHsl::egfp also showed in adult fat body tissue increased abundance on LDs under starvation. A general absence of overexpressed DmHsl::GFP on LDs in starved plin $1^{1}$ flies could not be seen but large lipid droplets were omitted. Whether this is caused by the absence of Plin1 directly, due to a missing interaction partner for $\mathrm{DmHsl}$ or an indirect effect caused by changed physicochemical properties of large LDs remains to be answered. Though overexpressed DmHsl::egfp was used for in vivo localization studies in larvae (Bi et al., 2012) and adults, DmHsl could also be found on embryonic LDs (Cermelli et al., 2006) and on induced LDs in S2 cells (Krahmer et al., 2013). Apart from that DmHsl was not found on LDs in fed larvae (Beller et al., 2006, Sahu-Osen, 2015). As embryogenesis represents a starvation state and the larval stage a feeding state a conserved mechanism of $\mathrm{DmH}$ sl localizing to lipid droplets under catabolic conditions might explain the finding and absence in the different lipid droplet proteomic studies.

The high maternal mRNA contribution of $\mathrm{DmHsl}$ as well as the detection of $\mathrm{DmHsl}$ on embryonic LDs implied a possible impairment in fertility of $\left.D m H s\right|^{1}$ flies. $D m H s l^{1}$ flies showed no noticeable difference during general stock keeping compared to control flies. Consistently, a fecundity assay revealed no changes between control and $D m H s I^{1}$ flies. Although male $\mathrm{HSL}^{-1-}$ mice were sterile due to gonadal hypotrophy and oligospermia (Osuga et al., 2000), DmHs/ ${ }^{1}$ male flies generated similar numbers of offspring with $\mathrm{DmHs}{ }^{1}$ and control females. However, fecundity was analyzed under laboratory terms providing ideal conditions for propagation. Therefore, a DmHsl deficiency might only be detrimental under wildtype living conditions. Also, the data indicates again a possible redundancy of $\mathrm{DmHsl}$ function as, despite the maternal DmHsl mRNA contribution, a DmHsl deficiency does not affect survival rate of embryos significantly.

When comparing the two available $\mathrm{DmHs} /$ deficient fly stocks ( $\mathrm{DmHs} \mathrm{I}^{1}$ and $\mathrm{DmHs}{ }^{b 24}$ ) possible differences should be addressed. Of course studies on $\mathrm{DmHs}^{\mathrm{b24}}$ mutants were restricted to larvae but these mutants showed TAG mobilization defects as well 
as increased body fat storage in L3 larvae (Bi et al., 2012). Both effects could not be detected in $D m H s I^{1}$ adults. Therefore, the $D m H s{ }^{1}$ data should be verified with $\left.D m H_{s}\right|^{b 24}$ flies and a proper genetically matched control for this strain.

In summary, $\mathrm{DmHs}^{1}$ flies are homozygous viable and show no obvious alterations in lipid storage and mobilization which might be compensated by so far undetected proteins. Interestingly, no homolog for hormone-sensitive lipase homolog can be found in birds suggesting also an alternative way to mobilize DAGs. 


\section{Supplement 2}

\subsection{Characterization of Cyp1 (CG9916)}

The structure of lipid droplets can be divided into a core, consisting mostly of neutral lipids like TAGs or Cholesterol esters, and phospholipid monolayer into which proteins are embedded that control the access to the core. LD-associated proteins in Drosophila melanogaster like Brummer lipase (Zimmermann et al., 2004, Grönke et al., 2005) or perilipins (Greenberg et al., 1991, Beller et al., 2010) have clear orthologues in humans.

Perilipins represent the most abundant protein species on LDs. Whereas, in humans five different perilipins can be found flies only have two perilipins. Additionally, the partial redundancy of perilipins in humans makes it difficult to characterize these proteins. Therefore, perilipins can be studied easier in flies.

A Dmplin1 mutant (referred to as plin $1^{1}$ ) exhibits increased body fat storage in adults but not in larvae and adult flies are hyperphagic (Beller et al., 2010). Lipid droplet size distribution of plin $1^{1}$ is changed compared to control flies and LDs with diameters larger than $30 \mu \mathrm{m}$ (called giant LDs) can be found. Interestingly, giant LDs appear already during larval stage, persist and become predominant in adults (Beller et al., 2010). A loss of Dmplin2 (referred to as plin $2^{1}$ ), on the other hand, leads to reduced body fat storage, whereas an overexpression has a diametric effect (Grönke et al., 2003, Teixeira et al., 2003). The lipid storage of plin $1^{1}$, plin $2^{1}$ (double mutant) flies is also decreased compared to control flies with the preference to towards bigger LDs (Beller et al., 2010).

Based on the hypothesis that perilipins modulate the abundance of proteins on LDs and that the plin $1^{1}$ giant LD phenotype is the cause or consequence of an altered proteome, the proteome of LDs isolated from plin $1^{1}$, plin2 $2^{1}$, control flies and plin $1^{1}$, plin2 ${ }^{1}$ was analysed by Dr. Anita Sahu-Osen (former member of the BirnerGrünberger, lab Medical University, Graz) in cooperation with our lab (Kühnlein group, MPI-bpc, Göttingen). 
Anita Sahu could identify 71 proteins in LD isolates from plin $1^{1}$ larval fat body (SahuOsen, 2015). Abundance was increased for 3 proteins and 11 proteins were found less compared to control larvae.

The predicted Peptidyl-prolyl cis-trans isomerase (P25007), known as cyclophilin 1 (referred to as Cyp1; CG9916), exhibited the strongest up-regulation with a 20-fold increase in plin $1^{1}$ compared to control flies. As Cyp1 abundance was not altered in plin $2^{1}$ and plin $1^{1}$, plin $2^{1}$ it was hypothesized that Cyp1 is involved in lipid droplet size regulation.

\subsection{Cyp1::eGFP can be associated with LDs}

In order to confirm the high abundance of Cyp1 in plin $1^{1}$ a Cyp1::eGFP construct (generated by Dr. Anita Sahu and Dr. Ronald Kühnlein) was expressed in the larval fat body (FB-SNS>GAL4) of plin $1^{1}$ flies. A fraction of Cyp1::eGFP is loosely associated with lipid droplets in a dot-like pattern (Figure 48). Most of the overexpressed protein is located in the cytoplasm, often accumulates and generates clusters distant to lipid droplets (Figure 48). Additionally, Cyp1::eGFP produces ring-like structures with no overlap of lipid droplet staining. 

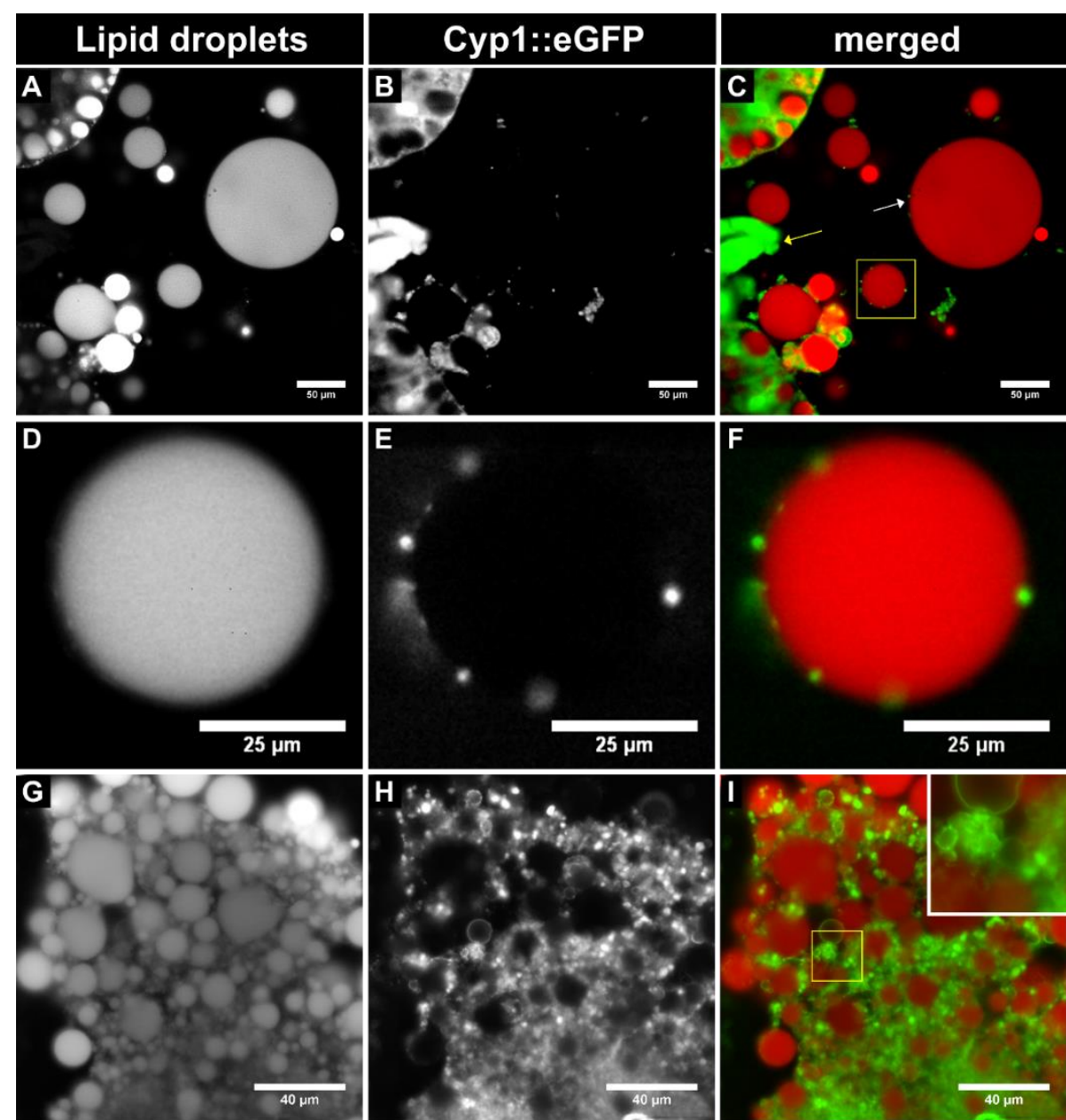

Figure 48 Cyp1::eGFP is loosely associated with lipid droplets. Cyp1::eGFP is expressed in larval fat body (FB-SNS>GAL4) of plin $1^{1}$ larvae. A fraction of the protein is associated in a dot-like pattern (white arrow, $C$ and magnified E, F). The major portion of Cyp1::eGFP generates aggregates (yellow arrow) or is localized in ring-like structures with no overlap with the LD staining (stained by LipidTOX ${ }^{\text {TM }}$ Deep Red).

\subsection{Average lipid droplet size is decreased in Cyp1 ${ }^{1}$ flies}

In order to characterize the biological function of Cyp1 a mutant was used (called Cyp $1^{1}$ ). A fly stock with a EP-insertion in the ORF of Cyp1 was available (Bloomington Drosophila Stock Center), backcrossed and homozygous stock was established by Ronald Kühnlein. An analysis from Anita Sahu-Osen already indicated a decrease in the average lipid droplet size during fat body targeted Cyp1-RNAi (Figure 49) that could be confirmed in Cyp1 $1^{1}$ fly fat body tissue (Figure 49). 

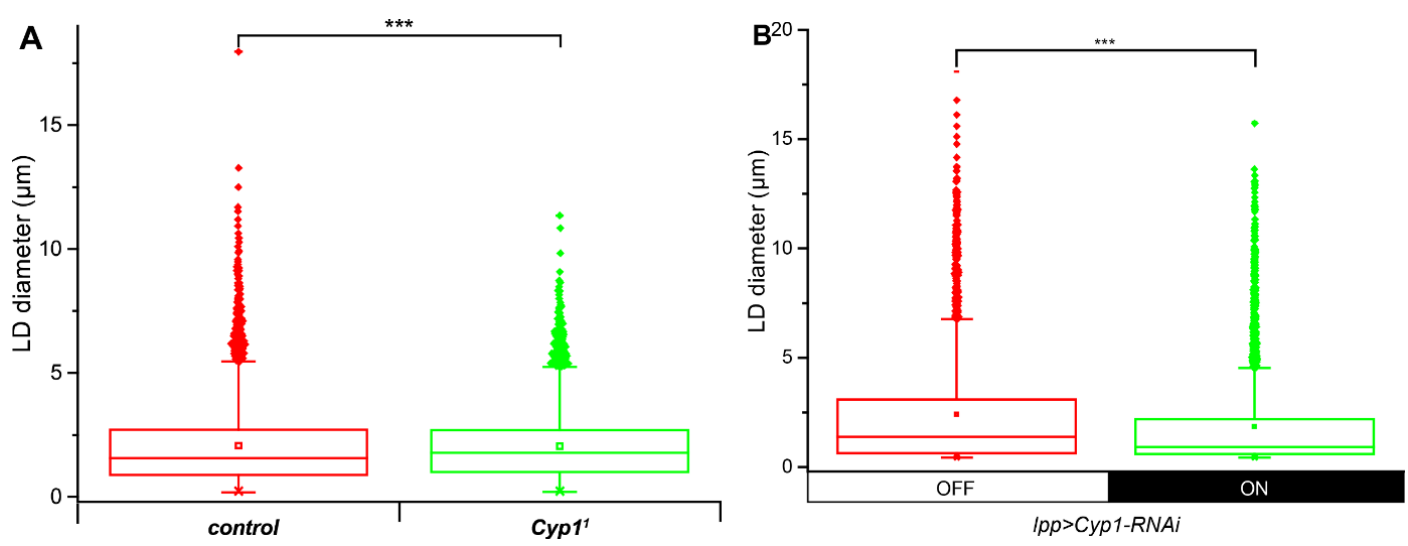

Figure 49 Average lipid droplet size (diameter) is reduced in Cyp1 ${ }^{1}$ and fat body targeted Cyp1-RNAi. Box plot of lipid droplet size quantified from confocal pictures of fluorescently stained lipid droplets in larval fat body cells. Center lines show the median, box limits indicate $25^{\text {th }}$ and $75^{\text {th }}$ percentiles as determined by OriginPro software; whiskers extend 1.5 times the interquartile range from the $25^{\text {th }}$ and $75^{\text {th }}$ percentiles (Mann-Whitney test; ${ }^{* * *}=P<0.001$; $n_{\text {lipid droplets }}$ analysed per genotype $>8000$ ).

\subsection{Body fat storage in $C_{y p} 1^{1}$ flies is not changed}

In order to see if the smaller average LD size also affects fat storage a CCA assay was performed. Smaller LD size had no effect on global lipid storage of Cyp $1^{1}$ L3 larvae.

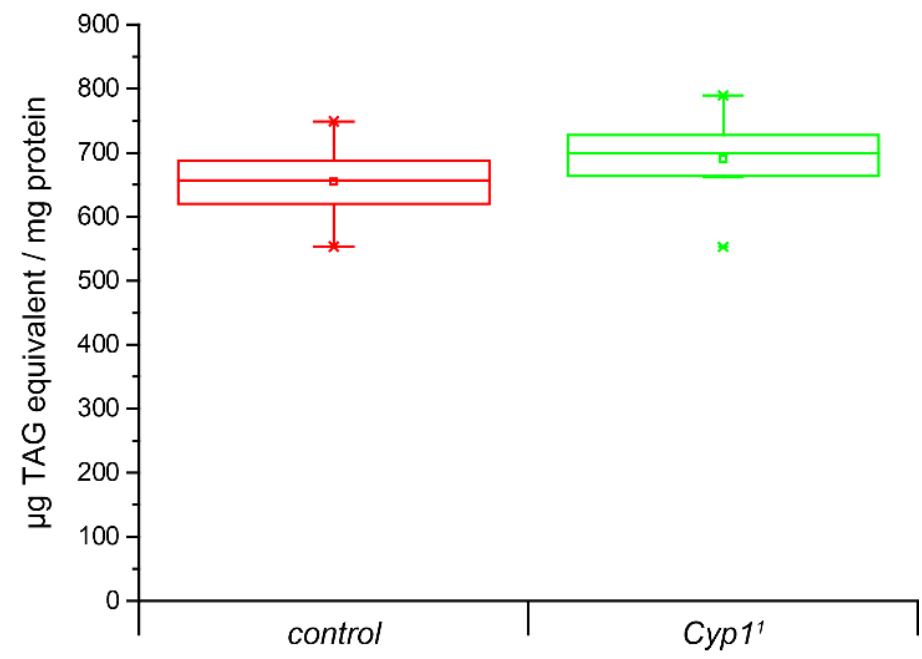

Figure 50 Body fat storage is unchanged in Cyp1 ${ }^{1}$ larvae (one-way ANOVA, $F_{(1,14)}=1.29, P=0.27$, Fisher $L S D P=0.27)$. Box plot of $\mu g$ TAG equivalent per mg protein measured with CCA assay. Center lines show the median, box limits indicate $25^{\text {th }}$ and $75^{\text {th }}$ percentiles as determined by OriginPro software; whiskers extend 1.5 times the interquartile range from the $25^{\text {th }}$ and $75^{\text {th }}$ percentiles. 


\subsection{Cyp1::eGFP expression in larvae reverts small LD phenotype}

As shown in Figure 48 Cyp1::eGFP is loosely connected to LDs. Average LD size is reduced in Cyp $1^{1}$ larval fat body cells. Therefore, it was tested if an overexpression of Cyp1::eGFP in the fat body of Cyp1 $1^{1}$ flies can reverse this phenotype.
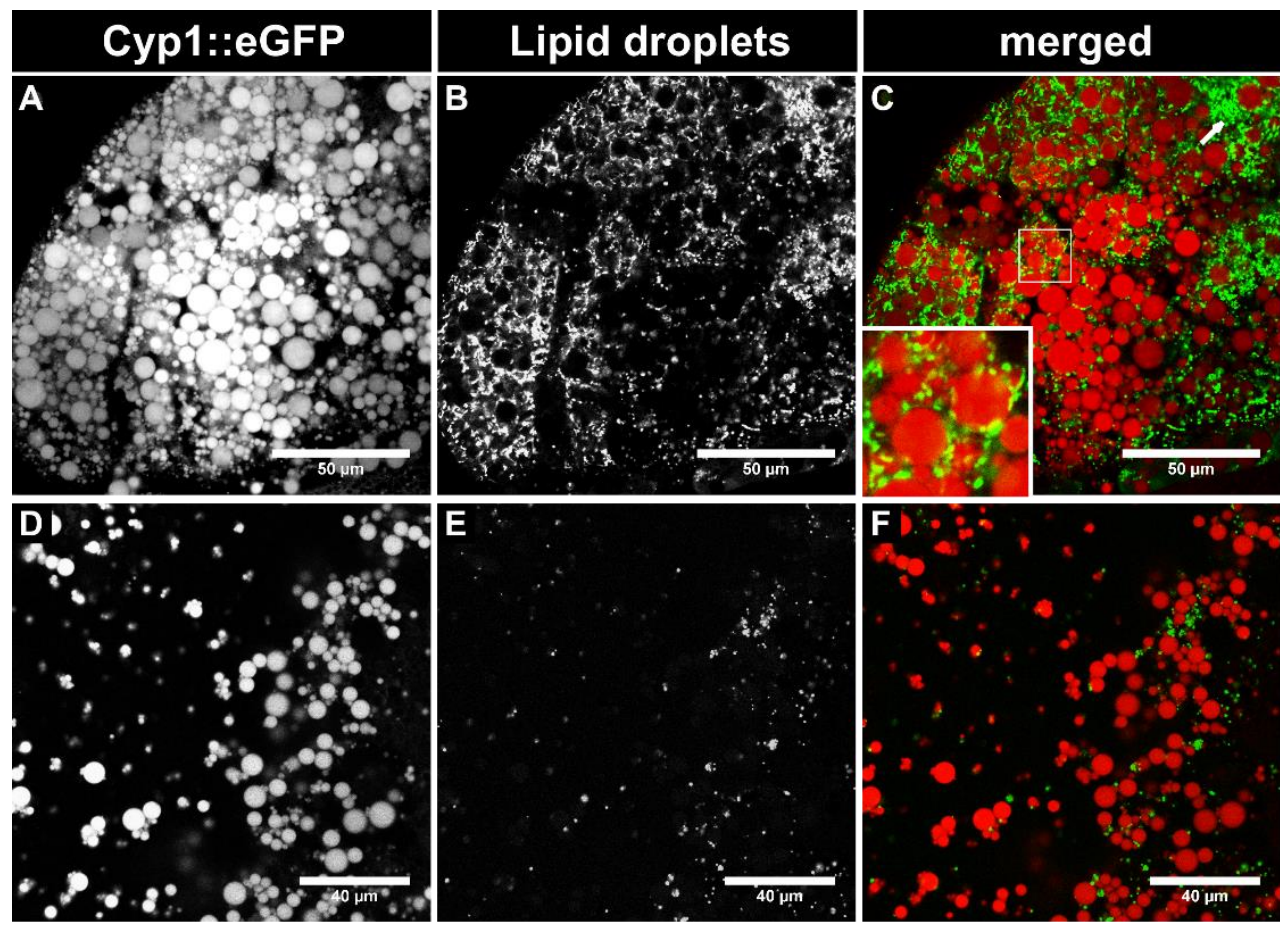

Figure 51 Cyp1::eGFP expression in Cyp1 ${ }^{1}$ larvae shows a dot-like distribution in close distance to lipid droplets (see white box) and non-lipid associated aggregates (arrow in C).

Indeed, expression of Cyp1::eGFP in the fat body (Lpp>GAL4) leads to a similar localization pattern (Figure 51) as shown in $p \operatorname{lin} 1^{1}$ (Figure 48) but upon release of LDs from the cells by mechanical cell disruption much less LDs were associated with Cyp1::eGFP. This correlates with the quantitative proteomics data from Dr. Anita Sahu were Cyp1 abundance was only elevated in plin $1^{1}$ mutants. Additionally, LD size quantification revealed a reversion of the reduced average LD size in $C y p 1^{1}$ towards bigger LD size (Figure 52). 


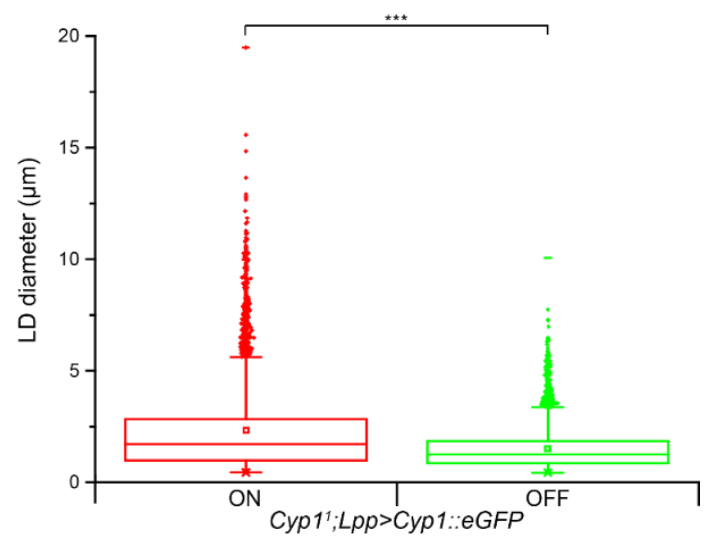

Figure 52 Expression of Cyp1::GFP in Cyp1 ${ }^{1}$ larvae (Lpp>GAL4) rescues small lipid droplet phenotype. Box plot of lipid droplet size quantified from confocal pictures of fluorescently stained lipid droplets in larval fat body cells. Center lines show the median, box limits indicate $25^{\text {th }}$ and $75^{\text {th }}$ percentiles as determined by OriginPro software; whiskers extend 1.5 times the interquartile range from the $25^{\text {th }}$ and $75^{\text {th }}$ percentiles (Mann-Whitney test; ${ }^{* * *}=P<0.001$; $n_{\text {lipid droplets }}$ analysed per genotype $>6000$ ).

\subsection{Cyp1::eGFP overexpression in larval fat body does not enhance giant LD phenotype of plin1 ${ }^{1}$ larvae}

A deficiency of Cyp1 leads to decreased average LD size and can be rescued by the expression of Cyp1::GFP. Therefore, it can be assumed that Cyp1 is directly involved in LD size regulation and that the Cyp1::GFP construct is functional. Increased protein abundance of Cyp1 correlates with the giant LD phenotype of plin $1^{1}$. Therefore, it was tested if the overexpression of Cyp1::eGFP in the fat body (FB-SNS>GAL4) in plin1 ${ }^{1}$ can enhance the giant LD phenotype.

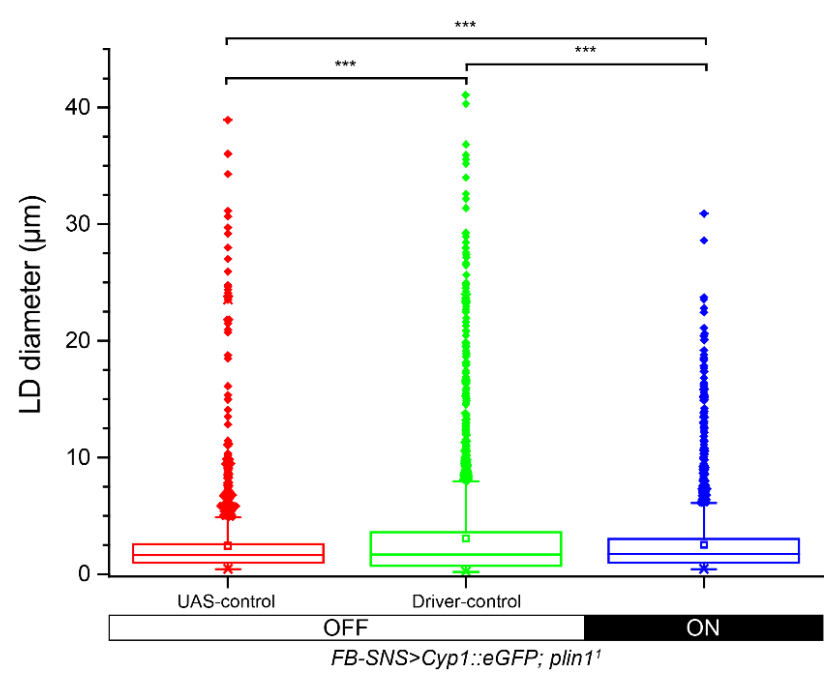

Figure 53 Overexpression of Cyp1::eGFP in plin $1^{1}$ Iarvae does not enhance plin $1^{1}$ giant LD phenotype. Box plot of lipid droplet size quantified from confocal pictures of fluorescently stained lipid droplets in larval fat body cells. Center lines show the median, box limits indicate $25^{\text {th }}$ and $75^{\text {th }}$ percentiles as determined by OriginPro software; whiskers extend 1.5 times the interquartile range from the $25^{\text {th }}$ and $75^{\text {th }}$ percentiles (Mann-Whitney test; ${ }^{* * *}=P<0.001 ; n_{\text {lipid droplets }}$ analysed per genotype $>1700$ ). 
Cyp1::eGFP could be found loosely associated with LDs (Figure 48) though endogenous Cyp1 is also expressed. Compared to control crosses, the average lipid droplet size in the fat body of larvae overexpressing Cyp1::eGFP did not shower bigger LDs nor was the average LD size significantly increased (Figure 53).

\subsection{Cyp1 contributes to lipid droplet size and storage lipid partioning in plin1 ${ }^{1}$ larvae}

Overexpression of Cyp1::eGFP did not enhance the giant LD phenotype. In order to designate the contribution of Cyp1 to the plin $1^{1}$ giant LD phenotype a double knock out mutant $\left(\right.$ Cyp $1^{1}$, plin1 $\left.1^{1}\right)$ was generated (by Dr. Ronald Kühnlein) and LD size was analysed in larval fat bodies (Figure 54).
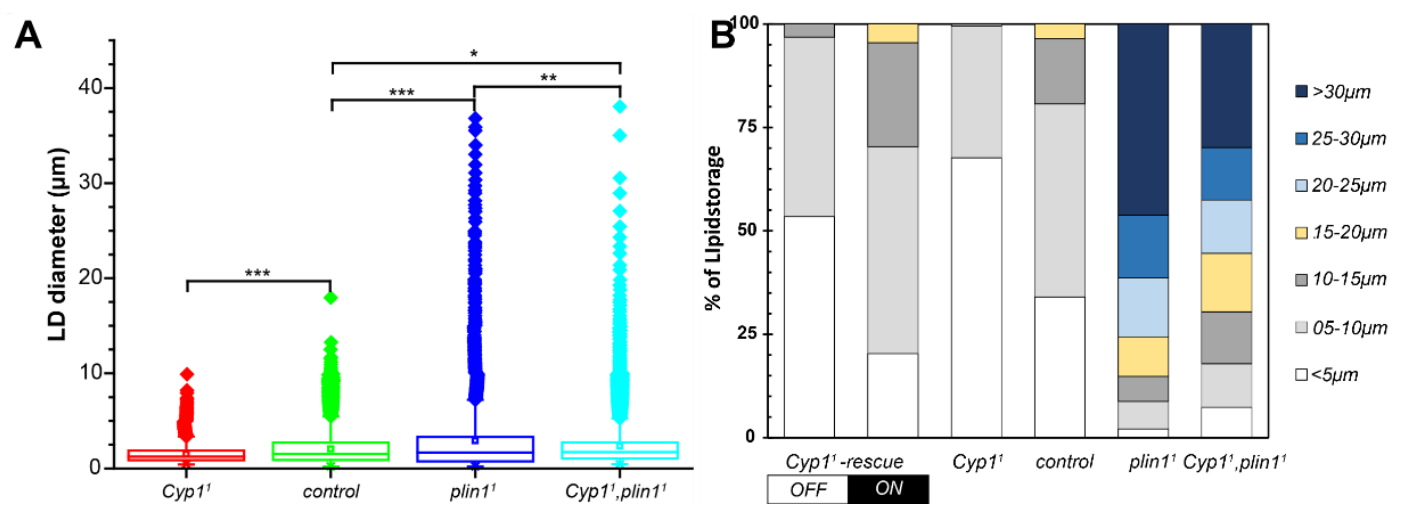

Figure 54 A double knockout of $C y p 1^{1}$, plin1 ${ }^{1}$ in larvae does not prevent the giant lipid droplet phenotype of plin1 ${ }^{1}$ larvae. (A) Box plot of lipid droplet size quantified from confocal pictures of fluorescently stained lipid droplets in larval fat body cells. Center lines show the median, box limits indicate $25^{\text {th }}$ and $75^{\text {th }}$ percentiles as determined by OriginPro software; whiskers extend 1.5 times the interquartile range from the $25^{\text {th }}$ and $75^{\text {th }}$ percentiles (Mann-Whitney test; ${ }^{* * *}=P<0.001, * *=P<0.01$, ${ }^{*}=P<0.05 ; n_{\text {lipid droplets }}$ analysed per genotype $>1700$ ). (B) Lipid storage partitioning reveals \% of amount of lipids stored in lipid droplets of the different sized bins: $<5,5-10,10-15,15-20,20-25,25-30,>30 \mu \mathrm{m}$.

The giant $L D$ phenotype of plin $1^{1}$ flies is characterized by the occurrence of LDs with more than $25 \mu \mathrm{m}$ in diameter (Figure 54). This does not mean that smaller LD sizes cannot be found anymore but that in general there is a shift towards larger LDs (Figure 54). Average LD size in $C y p 1^{1}$ is significantly reduced (Figure 54) and increased in plin $1^{1}$. A double knock out $\left(C y p 1^{1}, p l i n 1^{1}\right)$ shows an intermediate phenotype between controls and plin $1^{1}$. Giant LDs can still be observed in Cyp1,plin1 ${ }^{1}$ but total numbers of these LDs is less than in plin $1^{1}$. Additionally, the distribution of total lipid storage in lipid droplets larger than $20 \mu \mathrm{m}$ in diameter shifts from $75 \%$ to roughly $50 \%$ in $C y p 1^{1}$,plin $1^{1}$ double mutants. 


\subsection{Discussion}

In summary, Cyp1 is important for LD size modulation. A deficiency in Cyp1 directly leads to smaller LD size that can be rescued by the expression of Cyp1::eGFP. In a double knockout of $C y p 1^{1}, p l i n 1^{1}$ the absence of Cyp1 is not sufficient to prohibit the formation of larger LDs but reduces the severity of this phenotype. As an overexpression of Cyp1::eGFP does not enhance the LD size phenotype of plin $1^{1}$, it is very likely that Cyp1 alone is not the limiting factor for the size regulation but still plays an important role. Up to now it is not known through which mechanism Cyp1 modulates LD size. Cyclophilins exhibit a peptidyl-prolyl cis-trans isomerase (PPlase) activity that accelerates protein folding and provides them a chaperone-like function (Lodish and Kong, 1991, Stamnes et al., 1991, Steinmann et al., 1991, Kruse et al., 1995). Therefore, Cyp1 might help proteins necessary for lipid synthesis into LDs (Wilfling et al., 2013), directing fusions of LDs or shuttling of lipids between LDs or other cell organelles (e.g. ER) to get the right shape or stabilize complexes in order to perform these functions and finally leading to lipid droplet growth. In this context it is possible that Cyp1 is required for correct Plin1 folding to ensure a proper localization on LDs as Plin1 abundance correlates tightly with the available LD surface (Beller et al., 2010).

The abundance of Cyp1 in lipid droplet isolations from plin $1^{1}$ larval fat body cells is increased but Cyp1 was in principle detected in plin $2^{1}$ and control flies as well, indicating a general involvement of Cyp1 in LD growth that exceeds a somehow minimal defined size as the absence of Cyp1 does not inhibit LD generation. Also the results underline the importance of Plin1 regulating access of proteins to LDs and therefore modulating the size and lipolytic accessibility of storage lipids. Part of the function of Plin1 might be limiting the maximal LD size as the possible effects from having large LDs are not completely understood. Lipid mobilization in general is not impaired in plin $1^{1}$ and the body fat reduction during $\mathrm{bmm}$ overexpression is more pronounced than in plin $1^{+}$flies (Beller et al., 2010) overexpressing $b m m$ but possible additional side effects have not been addressed so far. 
Cyclophilins are evolutionary conserved between flies and humans and a BLAST search identifies HsCypA (Similarity: 84\% and Identity: 75\%) and HsCypD (79\% / 67\%) as potential functional homologs for DmCyp1. Both CypA and CypD are described to promote LD growth in their absence (by e.g. RNAi) in sg-1b replicon cells (express Hepatitis C virus proteins). A similar effect can be achieved via the inhibition of Cyclophilins with Cyclosporin A [NIM811] (Anderson et al., 2011). However, the mechanism by which CypA and CypD modulate LD size is not known. Studies in adult CypD ${ }^{-1}$ mice identified CypD to be important for the control of oxidative phosphorylation and the control of the mitochondrial permeability transition pore. CypD absence protected mitochondria from $\mathrm{Ca}^{2+}$ stress (Gainutdinov et al., 2015) but also altered the metabolism of mice. $C y p D^{-/-}$mice exhibited a higher metabolic rate with increased utilization of lipids (lower fat mass) and elevated body temperature. Additionally, CypD ${ }^{-/}$mice were protected from diet induced obesity (DevalarajaNarashimha et al., 2011, Taddeo et al., 2014) but older animals developed: insulin resistance, hyperglycaemia and glucose intolerance (Devalaraja-Narashimha et al., 2011). Therefore, cyclophilins are a promising group of largely uncharacterized proteins involved in metabolism.

DmCyp1 is the first cyclophilin in Drosophila that has been characterized with the previous (Sahu-Osen, 2015) and current work modulating LD size in larvae. Whereas, body fat storage in $C y p 1^{1}$ is not altered in larvae it remains to be addressed if this is also the case in adult flies. As only a portion of Cyp1::eGFP is loosely attached to LDs its exact localizations should be addressed in order to identify the underlying mechanism by which Cyp1 regulates LD size and to identify possible other biological processes it may modulates: e.g. metabolic rate, glycogen storage or glycolysis. Potential sides of residence are mitochondria that are often in close distance to LDs or the endoplasmic reticulum that has been described as a potential site for de novo lipid droplet synthesis (Zanghellini et al., 2010, Krahmer et al., 2011, Pol et al., 2014, Wilfling et al., 2014). 


\section{References}

Aguilera, C. M., Gil-Campos, M., Canete, R. \& Gil, A. 2008. Alterations in plasma and tissue lipids associated with obesity and metabolic syndrome. Clin Sci (Lond), 114, 183-93

Anderson, L. J., Lin, K., Compton, T. \& Wiedmann, B. 2011. Inhibition of cyclophilins alters lipid trafficking and blocks hepatitis C virus secretion. Virol J, 8, 329. PMC3138436

Arrese, E. L. \& Wells, M. A. 1994. Purification and properties of a phosphorylatable triacylglycerol lipase from the fat body of an insect, Manduca sexta. J Lipid Res, 35, $1652-60$

Ashrafi, K., Chang, F. Y., Watts, J. L., Fraser, A. G., Kamath, R. S., Ahringer, J. \& Ruvkun, G. 2003. Genome-wide RNAi analysis of Caenorhabditis elegans fat regulatory genes. Nature, 421, 268-72

Attrill, H., Falls, K., Goodman, J. L., Millburn, G. H., Antonazzo, G., Rey, A. J., Marygold, S. J. \& Flybase, C. 2016. FlyBase: establishing a Gene Group resource for Drosophila melanogaster. Nucleic Acids Res, 44, D786-92.PMC4702782

Barrio, L., Dekanty, A. \& Milan, M. 2014. MicroRNA-mediated regulation of Dp53 in the Drosophila fat body contributes to metabolic adaptation to nutrient deprivation. Cell Rep, 8, 528-41

Barry, W. E. \& Thummel, C. S. 2016. The Drosophila HNF4 nuclear receptor promotes glucosestimulated insulin secretion and mitochondrial function in adults. Elife, 5.PMC4869932

Bartz, R., Li, W. H., Venables, B., Zehmer, J. K., Roth, M. R., Welti, R., Anderson, R. G., Liu, P. \& Chapman, K. D. 2007. Lipidomics reveals that adiposomes store ether lipids and mediate phospholipid traffic. J Lipid Res, 48, 837-47

Bassett, A. R., Tibbit, C., Ponting, C. P. \& Liu, J. L. 2013. Highly efficient targeted mutagenesis of Drosophila with the CRISPR/Cas9 system. Cell Rep, 4, 220-8.PMC3714591

Bate, Martinez \& Arias 1993. The Development of Drosophila melanogaster, Cold Spring Harbour Laboratory Press.

Bauer, R., Voelzmann, A., Breiden, B., Schepers, U., Farwanah, H., Hahn, I., Eckardt, F., Sandhoff, K. \& Hoch, M. 2009. Schlank, a member of the ceramide synthase family controls growth and body fat in Drosophila. EMBO J, 28, 3706-16.PMC2790492

Baumbach, J., Hummel, P., Bickmeyer, I., Kowalczyk, K. M., Frank, M., Knorr, K., Hildebrandt, A., Riedel, D., Jäckle, H. \& Kühnlein, R. P. 2014a. A Drosophila in vivo screen identifies store-operated calcium entry as a key regulator of adiposity. Cell Metab, 19, 331-43

Baumbach, J., Xu, Y., Hehlert, P. \& Kühnlein, R. P. 2014b. Galphaq, Ggamma1 and PIc21C control Drosophila body fat storage. J Genet Genomics, 41, 283-92

Beller, M., Bulankina, A. V., Hsiao, H. H., Urlaub, H., Jackle, H. \& Kuhnlein, R. P. 2010. PERILIPIN-dependent control of lipid droplet structure and fat storage in Drosophila. Cell Metab, 12, 521-32

Beller, M., Riedel, D., Jansch, L., Dieterich, G., Wehland, J., Jäckle, H. \& Kühnlein, R. P. 2006 Characterization of the Drosophila lipid droplet subproteome. Mol Cell Proteomics, 5, 1082-94

Benzer, S. 1967. BEHAVIORAL MUTANTS OF Drosophila ISOLATED BY COUNTERCURRENT DISTRIBUTION. Proc Natl Acad Sci U S A, 58, 1112-9.PMC335755

Berg, J. M., Tymoczko, J. L. \& Stryer, L. 2007. Biochemie, Spektrum Akademischer Verlag. 
Berger, I., Fitzgerald, D. J. \& Richmond, T. J. 2004. Baculovirus expression system for heterologous multiprotein complexes. Nat Biotechnol, 22, 1583-7

Berridge, M. J. \& Oschman, J. L. 1969. A structural basis for fluid secretion by malpighian tubules. Tissue Cell, 1, 247-72

Bi, J., Xiang, Y., Chen, H., Liu, Z., Grönke, S., Kühnlein, R. P. \& Huang, X. 2012. Opposite and redundant roles of the two Drosophila perilipins in lipid mobilization. J Cell Sci, 125, 3568-77

Birse, R. T., Choi, J., Reardon, K., Rodriguez, J., Graham, S., Diop, S., Ocorr, K., Bodmer, R. \& Oldham, S. 2010. High-fat-diet-induced obesity and heart dysfunction are regulated by the TOR pathway in Drosophila. Cell Metab, 12, 533-44.PMC3026640

Bligh, E. G. \& Dyer, W. J. 1959. A rapid method of total lipid extraction and purification. Can J Biochem Physiol, 37, 911-7

Bodenstein, D. 1950. The postembryonic development of Drosophila, New York, Cold Spring Harbor Laboratory Press.

Boeszoermenyi, A., Nagy, H. M., Arthanari, H., Pillip, C. J., Lindermuth, H., Luna, R. E., Wagner, G., Zechner, R., Zangger, K. \& Oberer, M. 2015. Structure of a CGI-58 motif provides the molecular basis of lipid droplet anchoring. I Biol Chem, 290, 2636172.PMC4646293

Bohni, R., Riesgo-Escovar, J., Oldham, S., Brogiolo, W., Stocker, H., Andruss, B. F., Beckingham, K. \& Hafen, E. 1999. Autonomous control of cell and organ size by CHICO, a Drosophila homolog of vertebrate IRS1-4. Cell, 97, 865-75

Brand, A. H. \& Perrimon, N. 1993. Targeted Gene-Expression as a Means of Altering Cell Fates and Generating Dominant Phenotypes. Development, 118, 401-415

Brogiolo, W., Stocker, H., Ikeya, T., Rintelen, F., Fernandez, R. \& Hafen, E. 2001. An evolutionarily conserved function of the Drosophila insulin receptor and insulin-like peptides in growth control. Curr Biol, 11, 213-21

Broughton, S. J., Piper, M. D., Ikeya, T., Bass, T. M., Jacobson, J., Driege, Y., Martinez, P., Hafen, E., Withers, D. J., Leevers, S. J. \& Partridge, L. 2005. Longer lifespan, altered metabolism, and stress resistance in Drosophila from ablation of cells making insulinlike ligands. Proc Natl Acad Sci U S A, 102, 3105-10.PMC549445

Brown, J. M., Betters, J. L., Lord, C., Ma, Y., Han, X., Yang, K., Alger, H. M., Melchior, J., Sawyer, J., Shah, R., Wilson, M. D., Liu, X., Graham, M. J., Lee, R., Crooke, R., Shulman, G. I., Xue, B., Shi, H. \& Yu, L. 2010. CGI-58 knockdown in mice causes hepatic steatosis but prevents diet-induced obesity and glucose intolerance. J Lipid Res, 51, 330615.PMC2952571

Brown, J. M., Chung, S., Das, A., Shelness, G. S., Rudel, L. L. \& Yu, L. 2007. CGI-58 facilitates the mobilization of cytoplasmic triglyceride for lipoprotein secretion in hepatoma cells. J Lipid Res, 48, 2295-305

Buszczak, M., Lu, X., Segraves, W. A., Chang, T. Y. \& Cooley, L. 2002. Mutations in the midway gene disrupt a Drosophila acyl coenzyme A: diacylglycerol acyltransferase. Genetics, 160, 1511-8.PMC1462074

Canavoso, L. E., Jouni, Z. E., Karnas, K. J., Pennington, J. E. \& Wells, M. A. 2001. Fat metabolism in insects. Annu Rev Nutr, 21, 23-46 
Carvalho, M., Sampaio, J. L., Palm, W., Brankatschk, M., Eaton, S. \& Shevchenko, A. 2012. Effects of diet and development on the Drosophila lipidome. Mol Syst Biol, 8, 600.PMC3421444

Caviglia, J. M., Betters, J. L., Dapito, D. H., Lord, C. C., Sullivan, S., Chua, S., Yin, T., Sekowski, A., Mu, H., Shapiro, L., Brown, J. M. \& Brasaemle, D. L. 2011. Adipose-selective overexpression of ABHD5/CGI-58 does not increase lipolysis or protect against dietinduced obesity. J Lipid Res, 52, 2032-42.PMC3196235

Cerk, I. K., Salzburger, B., Boeszoermenyi, A., Heier, C., Pillip, C., Romauch, M., Schweiger, M., Cornaciu, I., Lass, A., Zimmermann, R., Zechner, R. \& Oberer, M. 2014. A peptide derived from G0/G1 switch gene 2 acts as noncompetitive inhibitor of adipose triglyceride lipase. J Biol Chem, 289, 32559-70.PMC4239610

Cermelli, S., Guo, Y., Gross, S. P. \& Welte, M. A. 2006. The lipid-droplet proteome reveals that droplets are a protein-storage depot. Curr Biol, 16, 1783-95

Chanarin, I., Patel, A., Slavin, G., Wills, E. J., Andrews, T. M. \& Stewart, G. 1975. Neutral-lipid storage disease: a new disorder of lipid metabolism. Br Med J, 1, 553-5.PMC1672681

Chien, S., Reiter, L. T., Bier, E. \& Gribskov, M. 2002. Homophila: human disease gene cognates in Drosophila. Nucleic Acids Res, 30, 149-51.PMC99119

Chintapalli, V. R., Al Bratty, M., Korzekwa, D., Watson, D. G. \& Dow, J. A. 2013. Mapping an atlas of tissue-specific Drosophila melanogaster metabolomes by high resolution mass spectrometry. PLoS One, 8, e78066.PMC3812166

Christensen, E., Hagve, T. A. \& Christophersen, B. O. 1986. Mitochondrial and peroxisomal oxidation of arachidonic and eicosapentaenoic acid studied in isolated liver cells. Biochim Biophys Acta, 879, 313-21

Contreras, J. A., Danielsson, B., Johansson, C., Osterlund, T., Langin, D. \& Holm, C. 1998. Human hormone-sensitive lipase: expression and large-scale purification from a baculovirus/insect cell system. Protein Expr Purif, 12, 93-9

Devalaraja-Narashimha, K., Diener, A. M. \& Padanilam, B. J. 2011. Cyclophilin D deficiency prevents diet-induced obesity in mice. FEBS Lett, 585, 677-82

Diangelo, J. R. \& Birnbaum, M. J. 2009. Regulation of fat cell mass by insulin in Drosophila melanogaster. Mol Cell Biol, 29, 6341-52.PMC2786867

Edgar, B. A. 2006. How flies get their size: genetics meets physiology. Nat Rev Genet, 7, 90716

Eichmann, T. O., Kumari, M., Haas, J. T., Farese, R. V., Jr., Zimmermann, R., Lass, A. \& Zechner, R. 2012. Studies on the substrate and stereo/regioselectivity of adipose triglyceride lipase, hormone-sensitive lipase, and diacylglycerol-O-acyltransferases. J Biol Chem, 287, 41446-57.PMC3510842

Farese, R. V., Jr. \& Walther, T. C. 2009. Lipid droplets finally get a little R-E-S-P-E-C-T. Cell, 139, 855-60.PMC3097139

Faubert, B., Boily, G., Izreig, S., Griss, T., Samborska, B., Dong, Z., Dupuy, F., Chambers, C., Fuerth, B. J., Viollet, B., Mamer, O. A., Avizonis, D., Deberardinis, R. J., Siegel, P. M. \& Jones, R. G. 2013. AMPK is a negative regulator of the Warburg effect and suppresses tumor growth in vivo. Cell Metab, 17, 113-24.PMC3545102

Faust, J. E., Manisundaram, A., Ivanova, P. T., Milne, S. B., Summerville, J. B., Brown, H. A., Wangler, M., Stern, M. \& Mcnew, J. A. 2014. Peroxisomes are required for lipid 
metabolism and muscle function in Drosophila melanogaster. PLoS One, 9, e100213.PMC4063865

Faust, J. E., Verma, A., Peng, C. \& Mcnew, J. A. 2012. An inventory of peroxisomal proteins and pathways in Drosophila melanogaster. Traffic, 13, 1378-92.PMC3443258

Fernando-Warnakulasuriya, J. P. G. \& Wells, M. A. 1988. Isolation and characterization of lipophorin fromDrosophila melanogaster larvae. Archives of Insect Biochemistry and Physiology, 8, 243-248

Foster, D. A. \& Toschi, A. 2009. Targeting mTOR with rapamycin: One dose does not fit all. Cell Cycle, 8, 1026-1029

Fredrikson, G., Stralfors, P., Nilsson, N. O. \& Belfrage, P. 1981. Hormone-Sensitive Lipase of Rat Adipose-Tissue - Purification and Some Properties. Journal of Biological Chemistry, 256, 6311-6320

Fredrikson, G., Tornqvist, H. \& Belfrage, P. 1986. Hormone-sensitive lipase and monoacylglycerol lipase are both required for complete degradation of adipocyte triacylglycerol. Biochimica Et Biophysica Acta (bba) - Lipids and Lipid Metabolism, 876, 288-293

Friedman, J. M. 2004. Modern science versus the stigma of obesity. Nat Med, 10, 563-9

Fullerton, M. D., Hakimuddin, F., Bonen, A. \& Bakovic, M. 2009. The development of a metabolic disease phenotype in CTP:phosphoethanolamine cytidylyltransferasedeficient mice. J Biol Chem, 284, 25704-13.PMC2757972

Gainutdinov, T., Molkentin, J. D., Siemen, D., Ziemer, M., Debska-Vielhaber, G., Vielhaber, S., Gizatullina, Z., Orynbayeva, Z. \& Gellerich, F. N. 2015. Knockout of cyclophilin D in Ppif(-)/(-) mice increases stability of brain mitochondria against $\mathrm{Ca}(2)(+)$ stress. Arch Biochem Biophys, 579, 40-6

Galikova, M., Diesner, M., Klepsatel, P., Hehlert, P., Xu, Y., Bickmeyer, I., Predel, R. \& Kühnlein, R. P. 2015. Energy Homeostasis Control in Drosophila Adipokinetic Hormone Mutants. Genetics, 201, 665-83.PMC4596676

Gelbart, W. M. \& Emmert, D. B. 2013. FlyBase High Throughput Expression Pattern Data.

Ghosh, A. K., Chauhan, N., Rajakumari, S., Daum, G. \& Rajasekharan, R. 2009. At4g24160, a soluble acyl-coenzyme A-dependent lysophosphatidic acid acyltransferase. Plant Physiol, 151, 869-81.PMC2754629

Ghosh, A. K., Ramakrishnan, G., Chandramohan, C. \& Rajasekharan, R. 2008a. CGI-58, the causative gene for Chanarin-Dorfman syndrome, mediates acylation of lysophosphatidic acid. J Biol Chem, 283, 24525-33.PMC3259832

Ghosh, A. K., Ramakrishnan, G. \& Rajasekharan, R. 2008b. YLR099C (ICT1) encodes a soluble Acyl-CoA-dependent lysophosphatidic acid acyltransferase responsible for enhanced phospholipid synthesis on organic solvent stress in Saccharomyces cerevisiae. J Biol Chem, 283, 9768-75.PMC2442289

Gilbert, L. I. \& Chino, H. 1974. Transport of lipids in insects. J Lipid Res, 15, 439-56

Gloor, G. B., Preston, C. R., Johnson-Schlitz, D. M., Nassif, N. A., Phillis, R. W., Benz, W. K., Robertson, H. M. \& Engels, W. R. 1993. Type I repressors of P element mobility. Genetics, 135, 81-95.PMC1205629

Grandison, R. C., Wong, R., Bass, T. M., Partridge, L. \& Piper, M. D. 2009. Effect of a standardised dietary restriction protocol on multiple laboratory strains of Drosophila melanogaster. PLoS One, 4, e4067.PMC2607010 
Granneman, J. G. 2015. Renaissance of brown adipose tissue research: integrating the old and new. Int J Obes Suppl, 5, S7-S10.PMC4850572

Granneman, J. G., Moore, H. P., Granneman, R. L., Greenberg, A. S., Obin, M. S. \& Zhu, Z. 2007. Analysis of lipolytic protein trafficking and interactions in adipocytes. $J$ Biol Chem, 282, 5726-35

Granneman, J. G., Moore, H. P., Krishnamoorthy, R. \& Rathod, M. 2009. Perilipin controls lipolysis by regulating the interactions of $A B$-hydrolase containing 5 (Abhd5) and adipose triglyceride lipase (Atgl). J Biol Chem, 284, 34538-44.PMC2787315

Greenberg, A. S., Egan, J. J., Wek, S. A., Garty, N. B., Blanchette-Mackie, E. J. \& Londos, C. 1991. Perilipin, a major hormonally regulated adipocyte-specific phosphoprotein associated with the periphery of lipid storage droplets. J Biol Chem, 266, 11341-6

Greene, J. C., Whitworth, A. J., Kuo, I., Andrews, L. A., Feany, M. B. \& Pallanck, L. J. 2003. Mitochondrial pathology and apoptotic muscle degeneration in Drosophila parkin mutants. Proc Natl Acad Sci U S A, 100, 4078-83.PMC153051

Grönke, S. 2005. Identification and characterization of genes controlling energy homeostasis in Drosophila melanogaster. Dr. rer. nat., Braunschweig.

Grönke, S., Beller, M., Fellert, S., Ramakrishnan, H., Jäckle, H. \& Kühnlein, R. P. 2003. Control of Fat Storage by a Drosophila PAT Domain Protein. Current Biology, 13, 603-606

Grönke, S., Clarke, D. F., Broughton, S., Andrews, T. D. \& Partridge, L. 2010. Molecular evolution and functional characterization of Drosophila insulin-like peptides. PLoS Genet, 6, e1000857.PMC2829060

Grönke, S., Mildner, A., Fellert, S., Tennagels, N., Petry, S., Müller, G., Jäckle, H. \& Kühnlein, R. P. 2005. Brummer lipase is an evolutionary conserved fat storage regulator in Drosophila. Cell Metab, 1, 323-30

Grönke, S., Müller, G., Hirsch, J., Fellert, S., Andreou, A., Haase, T., Jäckle, H. \& Kühnlein, R. P. 2007. Dual lipolytic control of body fat storage and mobilization in Drosophila. PLOS Biol, 5, e137.PMC1865564

Gruber, A., Cornaciu, I., Lass, A., Schweiger, M., Poeschl, M., Eder, C., Kumari, M., Schoiswohl, G., Wolinski, H., Kohlwein, S. D., Zechner, R., Zimmermann, R. \& Oberer, M. 2010. The N-terminal region of comparative gene identification-58 (CGI-58) is important for lipid droplet binding and activation of adipose triglyceride lipase. J Biol Chem, 285, 12289-98.PMC2852968

Guan, X. L., Cestra, G., Shui, G., Kuhrs, A., Schittenhelm, R. B., Hafen, E., Van Der Goot, F. G., Robinett, C. C., Gatti, M., Gonzalez-Gaitan, M. \& Wenk, M. R. 2013. Biochemical membrane lipidomics during Drosophila development. Dev Cell, 24, 98-111

Guo, Y., Walther, T. C., Rao, M., Stuurman, N., Goshima, G., Terayama, K., Wong, J. S., Vale, R. D., Walter, P. \& Farese, R. V. 2008. Functional genomic screen reveals genes involved in lipid-droplet formation and utilization. Nature, 453, 657-61.PMC2734507

Haemmerle, G., Moustafa, T., Woelkart, G., Buttner, S., Schmidt, A., Van De Weijer, T., Hesselink, M., Jaeger, D., Kienesberger, P. C., Zierler, K., Schreiber, R., Eichmann, T., Kolb, D., Kotzbeck, P., Schweiger, M., Kumari, M., Eder, S., Schoiswohl, G., Wongsiriroj, N., Pollak, N. M., Radner, F. P., Preiss-Landl, K., Kolbe, T., Rulicke, T., Pieske, B., Trauner, M., Lass, A., Zimmermann, R., Hoefler, G., Cinti, S., Kershaw, E. E., Schrauwen, P., Madeo, F., Mayer, B. \& Zechner, R. 2011. ATGL-mediated fat 
catabolism regulates cardiac mitochondrial function via PPAR-alpha and PGC-1. Nat Med, 17, 1076-85.PMC3244833

Haemmerle, G., Zimmermann, R., Hayn, M., Theussl, C., Waeg, G., Wagner, E., Sattler, W., Magin, T. M., Wagner, E. F. \& Zechner, R. 2002a. Hormone-sensitive lipase deficiency in mice causes diglyceride accumulation in adipose tissue, muscle, and testis. $J$ Biol Chem, 277, 4806-15

Haemmerle, G., Zimmermann, R., Strauss, J. G., Kratky, D., Riederer, M., Knipping, G. \& Zechner, R. 2002b. Hormone-sensitive lipase deficiency in mice changes the plasma lipid profile by affecting the tissue-specific expression pattern of lipoprotein lipase in adipose tissue and muscle. J Biol Chem, 277, 12946-52

Hardie, D. G. 2011. AMP-activated protein kinase: an energy sensor that regulates all aspects of cell function. Genes Dev, 25, 1895-908.PMC3185962

Harris, J. A. \& Benedict, F. G. 1918. A Biometric Study of Human Basal Metabolism. Proc Natl Acad Sci U S A, 4, 370-3.PMC1091498

Hartenstein, V. 1993. Atlas of Drosophila melanogaster Development, New York, Cold Spring Harbour Press.

Heier, C., Taschler, U., Radulovic, M., Aschauer, P., Eichmann, T. O., Grond, S., Wolinski, H., Oberer, M., Zechner, R., Kohlwein, S. D. \& Zimmermann, R. 2016. Monoacylglycerol Lipases Act as Evolutionarily Conserved Regulators of Non-oxidative Ethanol Metabolism. J Biol Chem, 291, 11865-75

Hildebrandt, A., Bickmeyer, I. \& Kühnlein, R. P. 2011. Reliable Drosophila body fat quantification by a coupled colorimetric assay. PLoS One, 6, e23796.PMC3170289

Hofer, P., Boeszoermenyi, A., Jaeger, D., Feiler, U., Arthanari, H., Mayer, N., Zehender, F., Rechberger, G., Oberer, M., Zimmermann, R., Lass, A., Haemmerle, G., Breinbauer, R., Zechner, R. \& Preiss-Landl, K. 2015. Fatty Acid-binding Proteins Interact with Comparative Gene Identification-58 Linking Lipolysis with Lipid Ligand Shuttling. J Biol Chem, 290, 18438-53.PMC4513104

Hofferek, V. 2016. Starvation Response of Drosophila melanogaster - a Lipidomic Approach. Dr. rer. nat., Universität Potsdam.

Holm, C., Olivecrona, G. \& Ottosson, M. 2001. Assays of lipolytic enzymes. Methods Mol Biol, $155,97-119$

Horiguchi, Y., Araki, M. \& Motojima, K. 2008. Identification and characterization of the ER/lipid droplet-targeting sequence in 17beta-hydroxysteroid dehydrogenase type 11. Arch Biochem Biophys, 479, 121-30

Huijsman, E., Van De Par, C., Economou, C., Van Der Poel, C., Lynch, G. S., Schoiswohl, G., Haemmerle, G., Zechner, R. \& Watt, M. J. 2009. Adipose triacylglycerol lipase deletion alters whole body energy metabolism and impairs exercise performance in mice. Am J Physiol Endocrinol Metab, 297, E505-13

Huttunen, J. K., Steinberg, D. \& Mayer, S. E. 1970. Protein kinase activation and phosphorylation of a purified hormone-sensitive lipase. Biochem Biophys Res Commun, 41, 1350-6

Igal, R. A. \& Coleman, R. 1996. Acylglycerol Recycling from Triacylglycerol to Phospholipid, Not Lipase Activity, Is Defective in Neutral Lipid Storage Disease Fibroblasts. J Biol Chem, 271, 16644-16651 
Ja, W. W., Carvalho, G. B., Mak, E. M., De La Rosa, N. N., Fang, A. Y., Liong, J. C., Brummel, T. \& Benzer, S. 2007. Prandiology of Drosophila and the CAFE assay. Proc Natl Acad Sci U $S$ A, 104, 8253-6.PMC1899109

James, C. N., Horn, P. J., Case, C. R., Gidda, S. K., Zhang, D., Mullen, R. T., Dyer, J. M., Anderson, R. G. \& Chapman, K. D. 2010. Disruption of the Arabidopsis CGI-58 homologue produces Chanarin-Dorfman-like lipid droplet accumulation in plants. Proc Natl Acad Sci U S A, 107, 17833-8.PMC2955100

Jenkins-Kruchten, A. E., Bennaars-Eiden, A., Ross, J. R., Shen, W. J., Kraemer, F. B. \& Bernlohr, D. A. 2003. Fatty acid-binding protein-hormone-sensitive lipase interaction. Fatty acid dependence on binding. J Biol Chem, 278, 47636-43

Jones, E. H., Harwood, L. J., Bowen, D. I. \& Griffiths, G. 1992. Lipid composition of subcellular membranes from larvae and prepupae of Drosophila melanogaster. Lipids, 27, 984987

Jones, R. G., Plas, D. R., Kubek, S., Buzzai, M., Mu, J., Xu, Y., Birnbaum, M. J. \& Thompson, C. B. 2005. AMP-activated protein kinase induces a p53-dependent metabolic checkpoint. Mol Cell, 18, 283-93

Kaluzny, M. A., Duncan, L. A., Merritt, M. V. \& Epps, D. E. 1985. Rapid seperation of lipid classes in high yield and purity using bonded phase columns. J Lipid Res., 26, 135-140

Katewa, S. D., Demontis, F., Kolipinski, M., Hubbard, A., Gill, M. S., Perrimon, N., Melov, S. \& Kapahi, P. 2012. Intramyocellular fatty-acid metabolism plays a critical role in mediating responses to dietary restriction in Drosophila melanogaster. Cell Metab, 16, 97-103.PMC3400463

Khaliullina, H., Bilgin, M., Sampaio, J. L., Shevchenko, A. \& Eaton, S. 2015. Endocannabinoids are conserved inhibitors of the Hedgehog pathway. Proc Natl Acad Sci U S A, 112, 3415-20.PMC4371992

Kim, S. J., Tang, T., Abbott, M., Viscarra, J. A., Wang, Y. \& Sul, H. S. 2016. AMPK Phosphorylates Desnutrin/ATGL and Hormone-Sensitive Lipase To Regulate Lipolysis and Fatty Acid Oxidation within Adipose Tissue. Mol Cell Biol, 36, 1961-76

Knittelfelder, O. L., Weberhofer, B. P., Eichmann, T. O., Kohlwein, S. D. \& Rechberger, G. N. 2014. A versatile ultra-high performance LC-MS method for lipid profiling. J Chromatogr B Analyt Technol Biomed Life Sci, 951-952, 119-28.PMC3946075

Krahmer, N., Guo, Y., Wilfling, F., Hilger, M., Lingrell, S., Heger, K., Newman, H. W., SchmidtSupprian, M., Vance, D. E., Mann, M., Farese, R. V., Jr. \& Walther, T. C. 2011. Phosphatidylcholine synthesis for lipid droplet expansion is mediated by localized activation of CTP:phosphocholine cytidylyltransferase. Cell Metab, 14, 50415.PMC3735358

Krahmer, N., Hilger, M., Kory, N., Wilfling, F., Stoehr, G., Mann, M., Farese, R. V., Jr. \& Walther, T. C. 2013. Protein correlation profiles identify lipid droplet proteins with high confidence. Mol Cell Proteomics, 12, 1115-26.PMC3650325

Kruse, M., Brunke, M., Escher, A., Szalay, A. A., Tropschug, M. \& Zimmermann, R. 1995. Enzyme assembly after de novo synthesis in rabbit reticulocyte lysate involves molecular chaperones and immunophilins. J Biol Chem, 270, 2588-94

Kuerschner, L., Moessinger, C. \& Thiele, C. 2008. Imaging of lipid biosynthesis: how a neutral lipid enters lipid droplets. Traffic, 9, 338-52 
Kühnlein, R. P. 2011. The contribution of the Drosophila model to lipid droplet research. Prog Lipid Res, 50, 348-56

Lafontan, M. \& Langin, D. 2009. Lipolysis and lipid mobilization in human adipose tissue. Prog Lipid Res, 48, 275-97

Lam, T. K., Schwartz, G. J. \& Rossetti, L. 2005. Hypothalamic sensing of fatty acids. Nat Neurosci, 8, 579-84

Lass, A., Zimmermann, R., Haemmerle, G., Riederer, M., Schoiswohl, G., Schweiger, M., Kienesberger, P., Strauss, J. G., Gorkiewicz, G. \& Zechner, R. 2006. Adipose triglyceride lipase-mediated lipolysis of cellular fat stores is activated by CGI-58 and defective in Chanarin-Dorfman Syndrome. Cell Metab, 3, 309-19

Lee, G. \& Park, J. H. 2004. Hemolymph sugar homeostasis and starvation-induced hyperactivity affected by genetic manipulations of the adipokinetic hormoneencoding gene in Drosophila melanogaster. Genetics, 167, 311-23.PMC1470856

Lee, H. C., Simon, G. M. \& Cravatt, B. F. 2015. ABHD4 regulates multiple classes of N-acyl phospholipids in the mammalian central nervous system. Biochemistry, 54, 253949.PMC4767004

Lefevre, C., Jobard, F., Caux, F., Bouadjar, B., Karaduman, A., Heilig, R., Lakhdar, H., Wollenberg, A., Verret, J. L., Weissenbach, J., Ozguc, M., Lathrop, M., Prud'homme, J. F. \& Fischer, J. 2001. Mutations in CGI-58, the gene encoding a new protein of the esterase/lipase/thioesterase subfamily, in Chanarin-Dorfman syndrome. Am J Hum Genet, 69, 1002-12.PMC1274347

Lim, D. H., Han, J. Y., Kim, J. R., Lee, Y. S. \& Kim, H. Y. 2012. Methionine sulfoxide reductase B in the endoplasmic reticulum is critical for stress resistance and aging in Drosophila. Biochem Biophys Res Commun, 419, 20-6

Liu, J., Wang, L., Harvey-White, J., Huang, B. X., Kim, H. Y., Luquet, S., Palmiter, R. D., Krystal, G., Rai, R., Mahadevan, A., Razdan, R. K. \& Kunos, G. 2008. Multiple pathways involved in the biosynthesis of anandamide. Neuropharmacology, 54, 17.PMC2219543

Lodish, F. H. \& Kong, N. 1991. Cyclosporin A inhibits an initial step in folding of transferrin within the endoplasmic reticulum. J Biol Chem, 266, 14835-14838

Londos, C., Brasaemle, D. L., Schultz, C. J., Segrest, J. P. \& Kimmel, A. R. 1999. Perilipins, ADRP, and other proteins that associate with intracellular neutral lipid droplets in animal cells. Semin Cell Dev Biol, 10, 51-8

Lord, C. C., Betters, J. L., Ivanova, P. T., Milne, S. B., Myers, D. S., Madenspacher, J., Thomas, G., Chung, S., Liu, M., Davis, M. A., Lee, R. G., Crooke, R. M., Graham, M. J., Parks, J. S., Brasaemle, D. L., Fessler, M. B., Brown, H. A. \& Brown, J. M. 2012. CGI-58/ABHD5derived signaling lipids regulate systemic inflammation and insulin action. Diabetes, 61, 355-63.PMC3266405

Marron, M. T., Markow, T. A., Kain, K. J. \& Gibbs, A. G. 2003. Effects of starvation and desiccation on energy metabolism in desert and mesic Drosophila. Journal of Insect Physiology, 49, 261-270

Massa, R., Pozzessere, S., Rastelli, E., Serra, L., Terracciano, C., Gibellini, M., Bozzali, M. \& Arca, M. 2016. Neutral lipid-storage disease with myopathy and extended phenotype with novel PNPLA2 mutation. Muscle Nerve, 53, 644-8 
Mcgettigan, J., Mclennan, R. K., Broderick, K. E., Kean, L., Allan, A. K., Cabrero, P., Regulski, M. R., Pollock, V. P., Gould, G. W., Davies, S. A. \& Dow, J. A. 2005. Insect renal tubules constitute a cell-autonomous immune system that protects the organism against bacterial infection. Insect Biochem Mol Biol, 35, 741-54

Mcmahon, D., Dinh, A., Kurz, D., Shah, D., Han, G. S., Carman, G. M. \& Brasaemle, D. 2014a. Comparative Gene Identification 58 (CGI-58)/Alpha Beta Hydrolase Domain 5 (ABHD5) Lacks Lysophosphatidic Acid Acyltransferase Activity. J Lipid Res, 55, 17501761

Mcmahon, D., Dinh, A., Kurz, D., Shah, D., Han, G. S., Carman, G. M. \& Brasaemle, D. L. 2014b. Comparative gene identification 58/alpha/beta hydrolase domain 5 lacks lysophosphatidic acid acyltransferase activity. J Lipid Res, 55, 17501761.PMC4109769

Montero-Moran, G., Caviglia, J. M., Mcmahon, D., Rothenberg, A., Subramanian, V., Xu, Z., Lara-Gonzalez, S., Storch, J., Carman, G. M. \& Brasaemle, D. L. 2010. CGI-58/ABHD5 is a coenzyme A-dependent lysophosphatidic acid acyltransferase. J Lipid Res, 51, 709-19.PMC2842141

Muccioli, G. G., Naslain, D., Backhed, F., Reigstad, C. S., Lambert, D. M., Delzenne, N. M. \& Cani, P. D. 2010. The endocannabinoid system links gut microbiota to adipogenesis. Mol Syst Biol, 6, 392.PMC2925525

Mukhopadhyay, S., Saqcena, M., Chatterjee, A., Garcia, A., Frias, M. A. \& Foster, D. A. 2015. Reciprocal regulation of AMP-activated protein kinase and phospholipase D. J Biol Chem, 290, 6986-93.PMC4358122

Musselman, L. P., Fink, J. L., Narzinski, K., Ramachandran, P. V., Hathiramani, S. S., Cagan, R. L. \& Baranski, T. J. 2011. A high-sugar diet produces obesity and insulin resistance in wild-type Drosophila. Dis Model Mech, 4, 842-9.PMC3209653

Musselman, L. P., Fink, J. L., Ramachandran, P. V., Patterson, B. W., Okunade, A. L., Maier, E., Brent, M. R., Turk, J. \& Baranski, T. J. 2013. Role of fat body lipogenesis in protection against the effects of caloric overload in Drosophila. J Biol Chem, 288, 802842.PMC3605622

Nagy, H. M., Paar, M., Heier, C., Moustafa, T., Hofer, P., Haemmerle, G., Lass, A., Zechner, R., Oberer, M. \& Zimmermann, R. 2014. Adipose triglyceride lipase activity is inhibited by long-chain acyl-coenzyme A. Biochim Biophys Acta, 1841, 588-94.PMC3988850

Nakayama, M., Sato, H., Okuda, T., Fujisawa, N., Kono, N., Arai, H., Suzuki, E., Umeda, M., Ishikawa, H. O. \& Matsuno, K. 2011. Drosophila carrying pex3 or pex16 mutations are models of Zellweger syndrome that reflect its symptoms associated with the absence of peroxisomes. PLoS One, 6, e22984.PMC3149631

Nelliot, A., Bond, N. \& Hoshizaki, D. K. 2006. Fat-body remodeling in Drosophila melanogaster. Genesis, 44, 396-400

Noyes, B. E., Katz, F. N. \& Schaffer, M. H. 1995. Identification and expression of the Drosophila adipokinetic hormone gene. Mol Cell Endocrinol, 109, 133-41

O'kane, C. J. 2003. Modelling human diseases in Drosophila and Caenorhabditis. Semin Cell Dev Biol, 14, 3-10

Oberer, M., Boeszoermenyi, A., Nagy, H. M. \& Zechner, R. 2011. Recent insights into the structure and function of comparative gene identification-58. Curr Opin Lipidol, 22, 149-58 
Ohsaki, Y., Cheng, J., Suzuki, M., Shinohara, Y., Fujita, A. \& Fujimoto, T. 2009. Biogenesis of cytoplasmic lipid droplets: from the lipid ester globule in the membrane to the visible structure. Biochim Biophys Acta, 1791, 399-407

Osuga, J., Ishibashi, S., Oka, T., Yagyu, H., Tozawa, R., Fujimoto, A., Shionoiri, F., Yahagi, N., Kraemer, F. B., Tsutsumi, O. \& Yamada, N. 2000. Targeted disruption of hormonesensitive lipase results in male sterility and adipocyte hypertrophy, but not in obesity. Proc Natl Acad Sci U S A, 97, 787-792.PMC15409

Ou, J., Miao, H., Ma, Y., Guo, F., Deng, J., Wei, X., Zhou, J., Xie, G., Shi, H., Xue, B., Liang, H. \& $\mathrm{Yu}, \mathrm{L}$. 2014. Loss of abhd5 promotes colorectal tumor development and progression by inducing aerobic glycolysis and epithelial-mesenchymal transition. Cell Rep, 9, 1798-811.PMC4268306

Pagnon, J., Matzaris, M., Stark, R., Meex, R. C., Macaulay, S. L., Brown, W., O'brien, P. E., Tiganis, T. \& Watt, M. J. 2012. Identification and functional characterization of protein kinase A phosphorylation sites in the major lipolytic protein, adipose triglyceride lipase. Endocrinology, 153, 4278-89

Palanker, L., Tennessen, J. M., Lam, G. \& Thummel, C. S. 2009. Drosophila HNF4 regulates lipid mobilization and beta-oxidation. Cell Metab, 9, 228-39.PMC2673486

Palm, W., Sampaio, J. L., Brankatschk, M., Carvalho, M., Mahmoud, A., Shevchenko, A. \& Eaton, S. 2012. Lipoproteins in Drosophila melanogaster--assembly, function, and influence on tissue lipid composition. PLoS Genet, 8, e1002828.PMC3406001

Panakova, D., Sprong, H., Marois, E., Thiele, C. \& Eaton, S. 2005. Lipoprotein particles are required for Hedgehog and Wingless signalling. Nature, 435, 58-65

Papadopoulou, D., Bianchi, M. W. \& Bourouis, M. 2004. Functional studies of shaggy/glycogen synthase kinase 3 phosphorylation sites in Drosophila melanogaster. Mol Cell Biol, 24, 4909-19.PMC416399

Park, S., Alfa, R. W., Topper, S. M., Kim, G. E., Kockel, L. \& Kim, S. K. 2014. A genetic strategy to measure circulating Drosophila insulin reveals genes regulating insulin production and secretion. PLoS Genet, 10, e1004555.PMC4125106

Park, S., Gidda, S. K., James, C. N., Horn, P. J., Khuu, N., Seay, D. C., Keereetaweep, J., Chapman, K. D., Mullen, R. T. \& Dyer, J. M. 2013. The alpha/beta hydrolase CGI-58 and peroxisomal transport protein PXA1 coregulate lipid homeostasis and signaling in Arabidopsis. Plant Cell, 25, 1726-39.PMC3694702

Partridge, L., Alic, N., Bjedov, I. \& Piper, M. D. 2011. Ageing in Drosophila: the role of the insulin/Igf and TOR signalling network. Exp Gerontol, 46, 376-81.PMC3087113

Patel, R. T., Soulages, J. L., Hariharasundaram, B. \& Arrese, E. L. 2005. Activation of the lipid droplet controls the rate of lipolysis of triglycerides in the insect fat body. J Biol Chem, 280, 22624-31

Pennington, E. J. \& Wells, M. A. 2002. Triacylglycerol-rich lipophorins are found in the dipteran infraorder culicomorpha, not just in mosquitoes. Journal of Insect Science, 2, 1-5

Pfaffl, M. W., Horgan, G. W. \& Dempfle, L. 2002. Relative expression software tool (REST (c)) for group-wise comparison and statistical analysis of relative expression results in real-time PCR. Nucleic Acids Research, 30, 1-10

Pol, A., Gross, S. P. \& Parton, R. G. 2014. Review: biogenesis of the multifunctional lipid droplet: lipids, proteins, and sites. J Cell Biol, 204, 635-46.PMC3941045 
Ponton, F., Chapuis, M. P., Pernice, M., Sword, G. A. \& Simpson, S. J. 2011. Evaluation of potential reference genes for reverse transcription-qPCR studies of physiological responses in Drosophila melanogaster. J Insect Physiol, 57, 840-50

Postic, C., Dentin, R., Denechaud, P. D. \& Girard, J. 2007. ChREBP, a transcriptional regulator of glucose and lipid metabolism. Annu Rev Nutr, 27, 179-92

Pribasnig, M. A., Mrak, I., Grabner, G. F., Taschler, U., Knittelfelder, O., Scherz, B., Eichmann, T. O., Heier, C., Grumet, L., Kowaliuk, J., Romauch, M., Holler, S., Anderl, F., Wolinski, H., Lass, A., Breinbauer, R., Marsche, G., Brown, J. M. \& Zimmermann, R. 2015. alpha/beta Hydrolase Domain-containing 6 (ABHD6) Degrades the Late Endosomal/Lysosomal Lipid Bis(monoacylglycero)phosphate. J Biol Chem, 290, 29869-81.PMC4705992

Radner, F. P., Grond, S., Haemmerle, G., Lass, A. \& Zechner, R. 2011. Fat in the skin: Triacylglycerol metabolism in keratinocytes and its role in the development of neutral lipid storage disease. Dermatoendocrinol, 3, 77-83.PMC3117006

Radner, F. P., Streith, I. E., Schoiswohl, G., Schweiger, M., Kumari, M., Eichmann, T. O., Rechberger, G., Koefeler, H. C., Eder, S., Schauer, S., Theussl, H. C., Preiss-Landl, K., Lass, A., Zimmermann, R., Hoefler, G., Zechner, R. \& Haemmerle, G. 2010. Growth retardation, impaired triacylglycerol catabolism, hepatic steatosis, and lethal skin barrier defect in mice lacking comparative gene identification-58 (CGI-58). J Biol Chem, 285, 7300-11.PMC2844178

Randle, P. J., Garland, P. B., Hales, C. N. \& Newsholme, E. A. 1963. The glucose fatty-acid cycle. Its role in insulin sensitivity and the metabolic disturbances of diabetes mellitus. Lancet, 1, 785-9

Reiter, L. T., Potocki, L., Chien, S., Gribskov, M. \& Bier, E. 2001. A systematic analysis of human disease-associated gene sequences in Drosophila melanogaster. Genome Res, 11, 1114-25.PMC311089

Rosenberg, J. 2012. Phänotypische Charakterisierung des dem human CGIß58 homologen Drosophila Gens CG1882. Bachelor of Science, University of Göttingen.

Rulifson, E. J., Kim, S. K. \& Nusse, R. 2002. Ablation of insulin-producing neurons in flies: growth and diabetic phenotypes. Science, 296, 1118-20

Sahu-Osen, A. 2015. Regulation of intracellular lipid storage and mobilization. Ph.D., Medical University of Graz.

Sahu-Osen, A., Montero-Moran, G., Schittmayer, M., Fritz, K., Dinh, A., Chang, Y. F., Mcmahon, D., Boeszoermenyi, A., Cornaciu, I., Russell, D., Oberer, M., Carman, G. M., Birner-Gruenberger, R. \& Brasaemle, D. L. 2015. CGI-58/ABHD5 is phosphorylated on Ser239 by protein kinase A: control of subcellular localization. $J$ Lipid Res, 56, 109-21.PMC4274058

Schweiger, M., Eichmann, T. O., Taschler, U., Zimmermann, R., Zechner, R. \& Lass, A. 2014. Measurement of lipolysis. Methods Enzymol, 538, 171-93.PMC4018506

Schweiger, M., Paar, M., Eder, C., Brandis, J., Moser, E., Gorkiewicz, G., Grond, S., Radner, F. P., Cerk, I., Cornaciu, I., Oberer, M., Kersten, S., Zechner, R., Zimmermann, R. \& Lass, A. 2012. G0/G1 switch gene-2 regulates human adipocyte lipolysis by affecting activity and localization of adipose triglyceride lipase. J Lipid Res, 53, 230717.PMC3466000 
Seedorf, U., Brysch, P., Engel, T., Schrage, K. \& Assmann, G. 1994. Sterol Carrier ProteinX Is Peroxisomal 3-Oxoacyl Coenzyme A Thiolase with Intrinsic Sterol Carrier and Lipid Transfer Activity. J Biol Chem, 269, 21277-21283

Sharma, A. M. \& Staels, B. 2007. Review: Peroxisome proliferator-activated receptor gamma and adipose tissue--understanding obesity-related changes in regulation of lipid and glucose metabolism. J Clin Endocrinol Metab, 92, 386-95

Smirnova, E., Goldberg, E. B., Makarova, K. S., Lin, L., Brown, W. J. \& Jackson, C. L. 2006. ATGL has a key role in lipid droplet/adiposome degradation in mammalian cells. $E M B O$ Rep, 7, 106-13.PMC1369222

Spandl, J., White, D. J., Peychl, J. \& Thiele, C. 2009. Live cell multicolor imaging of lipid droplets with a new dye, LD540. Traffic, 10, 1579-84

Speakman, J. R. 2004. Obesity: the integrated roles of environment and genetics. J Nutr, 134, 2090S-2105S

Spradling, A. C., Stern, D., Beaton, A., Rhem, E. J., Laverty, T., Mozden, N., Misra, S. \& Rubin, G. M. 1999. The Berkeley Drosophila Genome Project gene disruption project: Single P-element insertions mutating $25 \%$ of vital Drosophila genes. Genetics, 153, 13577.PMC1460730

Spradling, A. C., Stern, D. M., Kiss, I., Roote, J., Laverty, T. \& Rubin, G. M. 1995. Gene Disruptions Using P-Transposable Elements - an Integral Component of the Drosophila Genome Project. Proceedings of the National Academy of Sciences of the United States of America, 92, 10824-10830

Stamnes, A. M., Shieh, B.-H., Chuman, L., Harris, L. G. \& Zuker, S. C. 1991. The Cyclophilin Homolog ninaA is a Tissue-Specific Integral Membrane Protein Required for the Proper Synthesis of a Subset of Drosophila Rhodopsins. Cell, 65, 219-227

Steinberg, S. J., Dodt, G., Raymond, G. V., Braverman, N. E., Moser, A. B. \& Moser, H. W. 2006. Peroxisome biogenesis disorders. Biochim Biophys Acta, 1763, 1733-48

Steinmann, B., Bruckner, P. \& Superti-Furga, A. 1991. Cyclosporin A slows collagen triple-helix formation in vivo: indirect evidence for a physiologic role of peptidyl-prolyl cis-transisomerase. J Biol Chem, 266, 1299-303

Stergiopoulos, K., Cabrero, P., Davies, S. A. \& Dow, J. A. 2009. Salty dog, an SLC5 symporter, modulates Drosophila response to salt stress. Physiol Genomics, 37, 111.PMC2661102

Stone, J. V., Mordue, W., Batley, K. E. \& Morris, H. R. 1976. Structure of locust adipokinetic hormone, a neurohormone that regulates lipid utilisation during flight. Nature, 263, 207-11

Suzuki, Y., Shimozawa, N., Takahashi, Y., Imamura, A., Kondo, N. \& Orii, T. 1996. Peroxisomal disorders: clinical aspects. Ann N Y Acad Sci, 804, 442-9

Sztalryd, C. \& Kraemer, F. B. 1994. Differences in Hormone-Sensitive Lipase Expression in White Adipose-Tissue from Various Anatomic Locations of the Rat. MetabolismClinical and Experimental, 43, 241-247

Sztalryd, C., Xu, G., Dorward, H., Tansey, J. T., Contreras, J. A., Kimmel, A. R. \& Londos, C. 2003. Perilipin $A$ is essential for the translocation of hormone-sensitive lipase during lipolytic activation. J Cell Biol, 161, 1093-103.PMC2172984

Taddeo, E. P., Laker, R. C., Breen, D. S., Akhtar, Y. N., Kenwood, B. M., Liao, J. A., Zhang, M., Fazakerley, D. J., Tomsig, J. L., Harris, T. E., Keller, S. R., Chow, J. D., Lynch, K. R., 
Chokki, M., Molkentin, J. D., Turner, N., James, D. E., Yan, Z. \& Hoehn, K. L. 2014. Opening of the mitochondrial permeability transition pore links mitochondrial dysfunction to insulin resistance in skeletal muscle. Mol Metab, 3, 12434.PMC3953683

Takacs, A. C. 2007. Functionelle Charakterisierung des Drosophila melanogaster-Homologes des humanen Chanarin-Dorfman Syndrom Genes CGI-58. Diplomarbeit, Universität Göttingen.

Tansey, J. T., Huml, A. M., Vogt, R., Davis, K. E., Jones, J. M., Fraser, K. A., Brasaemle, D. L., Kimmel, A. R. \& Londos, C. 2003. Functional studies on native and mutated forms of perilipins. A role in protein kinase A-mediated lipolysis of triacylglycerols. J Biol Chem, 278, 8401-6

Tansey, J. T., Sztalryd, C., Gruia-Gray, J., Roush, D. L., Zee, J. V., Gavrilova, O., Reitman, M. L., Deng, C. X., Li, C., Kimmel, A. R. \& Londos, C. 2001. Perilipin ablation results in a lean mouse with aberrant adipocyte lipolysis, enhanced leptin production, and resistance to diet-induced obesity. Proc Natl Acad Sci U S A, 98, 6494-9.PMC33496

Tansey, J. T., Sztalryd, C., Hlavin, E. M., Kimmel, A. R. \& Londos, C. 2004. The central role of perilipin a in lipid metabolism and adipocyte lipolysis. IUBMB Life, 56, 379-85

Tatar, M., Kopelman, A., Epstein, D., Tu, M. P., Yin, C. M. \& Garofalo, R. S. 2001. A mutant Drosophila insulin receptor homolog that extends life-span and impairs neuroendocrine function. Science, 292, 107-10

Tauchi-Sato, K., Ozeki, S., Houjou, T., Taguchi, R. \& Fujimoto, T. 2002. The surface of lipid droplets is a phospholipid monolayer with a unique Fatty Acid composition. J Biol Chem, 277, 44507-12

Teixeira, L. S., Rabouille, C., Rørth, P., Ephrussi, A. \& Vanzo, N. F. 2003. Drosophila Perilipin/ADRP homologue Lsd2 regulates lipid metabolism. Mechanisms of Development, 120, 1071-1081

Tennessen, J. M., Barry, W. E., Cox, J. \& Thummel, C. S. 2014. Methods for studying metabolism in Drosophila. Methods, 68, 105-15.PMC4048761

Tortoriello, G., Rhodes, B. P., Takacs, S. M., Stuart, J. M., Basnet, A., Raboune, S., Widlanski, T. S., Doherty, P., Harkany, T. \& Bradshaw, H. B. 2013. Targeted lipidomics in Drosophila melanogaster identifies novel 2-monoacylglycerols and $\mathrm{N}$-acyl amides. PLoS One, 8, e67865.PMC3708943

Toschi, A., Lee, E., Xu, L., Garcia, A., Gadir, N. \& Foster, D. A. 2009. Regulation of mTORC1 and mTORC2 complex assembly by phosphatidic acid: competition with rapamycin. Mol Cell Biol, 29, 1411-20.PMC2648237

Trowitzsch, S., Bieniossek, C., Nie, Y., Garzoni, F. \& Berger, I. 2010. New baculovirus expression tools for recombinant protein complex production. J Struct Biol, 172, 4554

Ussher, J. R. \& Lopaschuk, G. D. 2009. Targeting malonyl CoA inhibition of mitochondrial fatty acid uptake as an approach to treat cardiac ischemia/reperfusion. Basic Res Cardiol, 104, 203-10

Vanhoe, F. G., Van Veldhoven, P. P., Fransen, M., Denis, S. \& Eyssen, J. H. 1993. The CoA Esters of 2-Methyl-branched Chain Fatty Acids and of the Bile Acid Intermediates Diand Trihydroxycoprostanic Acids Are Oxidized by One Single Peroxisomal Branched Chain Acyl-CoA Oxidase in Human Liver and Kidney. J Biol Chem, 268, 10335-10344 
Wanders, R. J., Van Roermund, C. W., Van Wijland, J. a. M., Schutgens, H. B. R., Heikoop, J., Van Den Bosch, H., Schram, W. A. \& Tager, M. J. 1987. Peroxisomal Fatty Acid BOxidation in Relation to the Accumulation of Very Long Chain Fatty Acids in Cultured Skin Fibroblasts from Patients with Zellweger Syndrome and Other Peroxisomal Disorders. J Clin Invest, 80, 1778-1783

Wang, B., Moya, N., Niessen, S., Hoover, H., Mihaylova, M. M., Shaw, R. J., Yates, J. R., 3rd, Fischer, W. H., Thomas, J. B. \& Montminy, M. 2011. A hormone-dependent module regulating energy balance. Cell, 145, 596-606.PMC3129781

Wessing, A. \& Eichelberg, D. 1969. Elektronenoptische Untersuchungen an den Nierentubuli (Malpighische Gefäße) von Drosophila melanogaster. Z. Zellforschung, 101, 285-322

Who 2013. Meeting of the Nutrition Knowledge Network. Meeting Report.

Who 2014. GLOBAL STATUS REPORT on noncommunicable diseases 2014. World Health Organization

Who 2016. Consideration of the evidence on childhood obesity for the Commission on Ending Childhood Obesity. World Health Organization

Wilfling, F., Haas, J. T., Walther, T. C. \& Farese, R. V., Jr. 2014. Lipid droplet biogenesis. Curr Opin Cell Biol, 29, 39-45.PMC4526149

Wilfling, F., Wang, H., Haas, J. T., Krahmer, N., Gould, T. J., Uchida, A., Cheng, J. X., Graham, M., Christiano, R., Frohlich, F., Liu, X., Buhman, K. K., Coleman, R. A., Bewersdorf, J., Farese, R. V., Jr. \& Walther, T. C. 2013. Triacylglycerol synthesis enzymes mediate lipid droplet growth by relocalizing from the ER to lipid droplets. Dev Cell, 24, 38499.PMC3727400

Xie, M. \& Roy, R. 2015. The Causative Gene in Chanarian Dorfman Syndrome Regulates Lipid Droplet Homeostasis in C. elegans. PLoS Genet, 11, e1005284.PMC4470697

Xu, X., Gopalacharyulu, P., Seppanen-Laakso, T., Ruskeepaa, A. L. B., Aye, C. C., Carson, B. P., Mora, S., Oresic, M. \& Teleman, A. A. 2012. Insulin signaling regulates fatty acid catabolism at the level of CoA activation. PLoS Genet, 8, e1002478.PMC3261918

Yamaguchi, T., Omatsu, N., Matsushita, S. \& Osumi, T. 2004. CGI-58 interacts with perilipin and is localized to lipid droplets. Possible involvement of CGI-58 mislocalization in Chanarin-Dorfman syndrome. J Biol Chem, 279, 30490-7

Yamaguchi, T., Omatsu, N., Morimoto, E., Nakashima, H., Ueno, K., Tanaka, T., Satouchi, K., Hirose, F. \& Osumi, T. 2007. CGI-58 facilitates lipolysis on lipid droplets but is not involved in the vesiculation of lipid droplets caused by hormonal stimulation. J Lipid Res, 48, 1078-89

Yatsenko, A. S., Marrone, A. K., Kucherenko, M. M. \& Shcherbata, H. R. 2014. Measurement of metabolic rate in Drosophila using respirometry. J Vis Exp, e51681

Yore, M. M., Syed, I., Moraes-Vieira, P. M., Zhang, T., Herman, M. A., Homan, E. A., Patel, R. T., Lee, J., Chen, S., Peroni, O. D., Dhaneshwar, A. S., Hammarstedt, A., Smith, U., Mcgraw, T. E., Saghatelian, A. \& Kahn, B. B. 2014. Discovery of a class of endogenous mammalian lipids with anti-diabetic and anti-inflammatory effects. Cell, 159, 31832.PMC4260972

Zanghellini, J., Wodlei, F. \& Von Grunberg, H. H. 2010. Phospholipid demixing and the birth of a lipid droplet. J Theor Biol, 264, 952-61

Zehmer, J. K., Huang, Y., Peng, G., Pu, J., Anderson, R. G. \& Liu, P. 2009. A role for lipid droplets in inter-membrane lipid traffic. Proteomics, 9, 914-21.PMC2676673 
Zimmermann, R., Strauss, J. G., Haemmerle, G., Schoiswohl, G., Birner-Gruenberger, R., Riederer, M., Lass, A., Neuberger, G., Eisenhaber, F., Hermetter, A. \& Zechner, R. 2004. Fat mobilization in adipose tissue is promoted by adipose triglyceride lipase. Science, 306, 1383-6

Zinke, I., Schutz, C. S., Katzenberger, J. D., Bauer, M. \& Pankratz, M. J. 2002. Nutrient control of gene expression in Drosophila: microarray analysis of starvation and sugardependent response. EMBO J, 21, 6162-73.PMC137192 


\section{Selbständigkeitserklärung}

Bestätigung zur eigenständigen Anfertigung der wissenschaftlichen Arbeit: Hiermit bestätige ich die folgende Dissertation mit dem Titel:

"Function of the $\alpha / \beta$-hydrolase fold family proteins Pummelig (CG1882) and hormone-sensitive lipase in the Drosophila melanogaster lipid metabolism "

selbstständig und ohne unerlaubte Hilfe/Hilfsmittel angefertigt zu haben.

(Philip Hehlert, Ort, Datum) 


\section{Promovierenden-Erklärung}

der Georg-August-Universität Göttingen

Name: Hehlert, Philip

Anschrift: Arndtstr. 1, 37075, Göttingen, Niedersachsen, Deutschland

Ich beabsichtige, eine Dissertation zum Thema

\section{Function of the $\alpha / \beta$-hydrolase fold family proteins Pummelig (CG1882) \\ and Hormone-sensitive lipase \\ in the Drosophila melanogaster lipid metabolism}

an der Georg-August-Universität Göttingen anzufertigen. Dabei werde ich von Herrn

Prof.....Ernst Wimmer..... betreut.

Ich gebe folgende Erklärung ab:

1. Die Gelegenheit zum vorliegenden Promotionsvorhaben ist mir nicht kommerziell vermittelt

worden. Insbesondere habe ich keine Organisation eingeschaltet, die gegen Entgelt Betreuerinnen

und Betreuer für die Anfertigung von Dissertationen sucht oder die mir obliegenden Pflichten hinsichtlich der Prüfungsleistungen für mich ganz oder teilweise erledigt.

2. Hilfe Dritter wurde bis jetzt und wird auch künftig nur in wissenschaftlich vertretbarem und prüfungsrechtlich zulässigem Ausmaß in Anspruch genommen. Insbesondere werden alle Teile der Dissertation selbst angefertigt; unzulässige fremde Hilfe habe ich dazu weder unentgeltlich noch

entgeltlich entgegengenommen und werde dies auch zukünftig so halten.

3. Die Richtlinien zur Sicherung der guten wissenschaftlichen Praxis an der Universität Göttingen werden von mir beachtet.

4. Eine entsprechende Promotion wurde an keiner anderen Hochschule im In- oder Ausland beantragt; die eingereichte Dissertation oder Teile von ihr wurden nicht für ein anderes Promotionsvorhaben verwendet.

Mir ist bekannt, dass unrichtige Angaben die Zulassung zur Promotion ausschließen bzw. später

zum Verfahrensabbruch oder zur Rücknahme des erlangten Grades führen.

Göttingen, den

(Unterschrift) 


\section{Curriculum vitae}

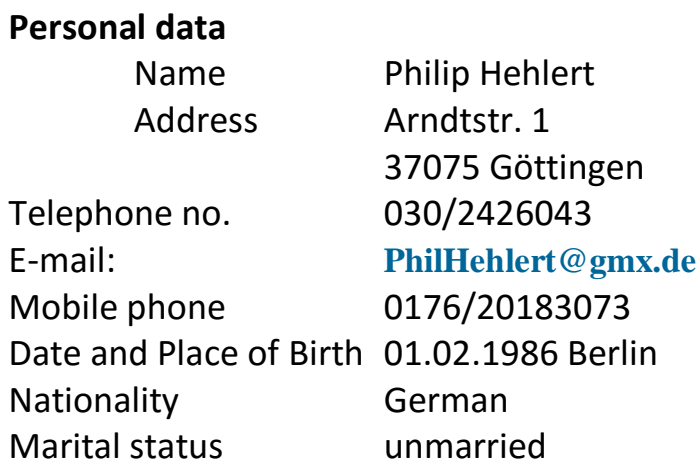

\section{Publications:}

Gáliková, M., Diesner, M., Klepsatel, P., Hehlert, P., Xu, Y., Bickmeyer, I., ... Kühnlein, R. P. (2015). Energy homeostasis control in Drosophila adipokinetic hormone mutants. Genetics, 201(2), 665-683. http://doi.org/10.1534/genetics.115.178897

Baumbach, J., Xu, Y., Hehlert, P., \& Kühnlein, R. P. (2014). Gaq, Gү1 and Plc21C control Drosophila body fat storage. Journal of Genetics and Genomics, 41(5), 283-292. http://doi.org/10.1016/j.jgg.201 4.03.005

Birner-Gruenberger, R., Bickmeyer, I., Lange, J., Hehlert, P., Hermetter, A., Kollroser, M., ... Kühnlein, R. P. (2012). Functional fat body proteomics and gene targeting reveal in vivo functions of Drosophila melanogaster $\alpha$-Esterase-7. Insect Biochemistry and Molecular Biology, 42(3), 220-9. http://doi.org/10.1016/j.ibmb.2011.12.004

\section{Conference Contribution}

German Fly Metabolism Meeting, 2015, University of Bonn; Oral presentation, Title: “Role of the $\alpha / \beta$-hydrolase fold family protein Pummelig in lipid metabolism"

\section{Education and working experience}

Since 06.2012 Doctoral Thesis, in the research group "Molecular Physiology" of Dr. Ronald Kühnlein in the Department "Molecular Development" at the "Max-Planck-Institute für biophysikalische Chemie", Göttingen, Enrolled at the "Georg-August-Universität Göttingen, GGNBProgram: Genes and Development

03.05.2012 Acquistion of Master of Science degree of Biotechnology at LUAS

degree: $\quad$ Master of Science

grade: $\quad 1,8$ (good)

23.02.2011-03.05.2012 Master thesis at the research group: Molecular Physiology (MPI-BPC; Dr. Ronald Kühnlein) | topic: „Dynamics in Lipid Metabolism: a CARS Approach"

grade: 
01.03.2012- Matriculated at Lausitz University of Applied Science (LUAS); Senftenberg, Master program: Biotechnology

18.02.2010 Acquisition of Bachelor of Science degree of Biotechnology at LUAS

degree: $\quad$ Bachelor of Science

grade: $\quad 2,2$ (good)

01.08.2009-01.02.2010 Bachelor thesis at the junior research group: Real-time-PCR" (Prof. Christian Schröder, Dr. Peter Schierack) | topic: "Molecular analysis of the AC16 cell line generated from human adult ventricular cardiomyocytes

$$
\text { grade: } \quad 1,6
$$

01.08.2008-01.03.2009 Practical term at MPI-CBG Dresden in the research group „Animal Models of Regeneration“ (Elly Tanaka) | topic: „Screening for genes specific for regeneration in Ambystoma mexicanum"

grade: $\quad 1,7$

Since 01.03.2008

2006

2005-2006

1998-2005

1996-1998

1992-1996
Tutor for younger students

matriculated at LUAS studies:
B.Sc. Biotechnologie

Conscript to Bundeswehr; Luftwaffensicherungssoldat Max-Planck Gymnasium Berlin-Mitte $\begin{array}{ll}\text { degree: } & \text { Baccalaureate } \\ \text { grade: } & 2,5\end{array}$

$9^{\text {th }}$ Primary school Berlin Mitte $2^{\text {nd }}$ Primary school Berlin Mitte

\section{Extracurricular education}

2005-2006

08.2005

11.2005

11.2004

11.2004
Graduated training's course for "Stationsausbilder" in the Bundeswehr Drivers licence class B

Participated at: Simulation of the European Parliament (SIMEP) for the $2^{\text {nd }}$ time

Participated at: Simulation of the European Parlament (SIMEP)

Attended a practical course at Gläsernes Labor Berlin

\section{Grants and Stipends}

- PhD Stipend from Max-Planck society (2012-2016)

- GGNB Travel Grant 2013

Language skills:

German

Englisch

Latein

\footnotetext{
- mother tongue

- very good reading, writing and verbal skills

- Latinum
} 


\section{Computing literacy}

- Organization and administration of Data-Storage system of the Research Group "Molecular Physiology"

- Windows and MacUser, limited experience with Linux

- Advanced knowledge in Word-, PowerPoint- and Excel

- Extended skills in Adobe Photoshop, Illustrator, Lightroom, InDesign

- Computer technics (Installation \& Repairs)

- Basics in bioinformatics and primer design

- Use of OriginPro

\section{Additonal activities}

- Student representative GGNB program Genes and Development (2014-2015)

- Student representative in Fachbereichsrat Bio- Chemie- und Verfahrenstechnik at LUAS

- Class representative in the $4^{\text {th }}$ and $6^{\text {th }}$ in primary school

- Engaged commitment in the public relations for the Max Plank Gymnasium

\section{Interests}

- Biology and medicine

- Volleyball (Active player in the "Mixed League Niedersachsen Süd" playing for "TSV Roringen", cycling (Attendee of "Tour de Energy" and "Berlin Velothon")

- Digital Photography (Canon, Fuji and Leica)

- Computer and technology

- Movies and cinema 


\section{Practical courses and laboratory skills}

\section{Practical courses during studies}

inorganic chemistry

organic chemistry

physical chemistry

physics

microbiology

bioprocess engineering

biochemistry

technical microbiology

gene technology

Project work: Immunohistochemical detection of proliferation markers Ki-67 and MCM6 in genetically modified high proliferating and normal chondrocytes under different oxygen-levels

Lab course: "Metabolic Analysis and Engineering “

Lab course: "Enzyme Technology"

Lab course: "Purification and Characterisation of Proteins"

Lab course: "Microbes as Macromolecule Factories"

Lab course: "Molecular biology: Principles, methods and applications"

\section{Microbiology}

- aseptic working under the flame and Laminar Air Flow

- Cultivation of different bacterial and fungal strands

- Isolation of pure fungal cultures from unsterile habitat samples

- Counting cells with THOMA-chamber

- Antibiotic sensitivity tests

- Cell preparation; Filtration, Centrifugation, French Press, working with liquid nitrogen

- Transforming bacteria, generation of chemo- und electro competent E-coli

\section{Biochemistry}

- SDS-Page

- Western-Blot

- FPLC und HPLC (His-tag und GST-tag purification)

- Bradford-Assay, BCA-Assay

- Coupled colorimetric assay (Glycerol measurement for fat determination)

- Thin layer chromatography

- Enzyme activity test via spectroscopy

- Trigylceride hydrolase assay + various neutral- and phospholipids

- Lipid extraction from Drosophila samples

- Lipid fractionation by solid phase extraction (SPE)

- Thin layer chromatography

\section{Cell culture}

- Passaging cells, Freezing, Thawing

- Cell-line used:

- AC16, human chondrocytes, mouse fibroblasts, Drosophila S2 and Kc-167, COS-7, SF-9, Hi5 
- Transfection of eukaryotic cells:

- Electroporation, hiPerfect Transfection Reagent Qiagen, siPORT ${ }^{\mathrm{TM}}$ Amin Transfection Agent (Ambion), Nanofectin (PAA), Chen\&Okayama (Calcium phosphate), $\mathrm{X}$ treme Gene

- $\quad$ FACS (PI-staining)

- Antibody-staining after Fixation

- 2D and 3D-cultivation of highly proliferating chondrocytes and AC16 cells

- lacZ-assay, BrdU-staining, GFP-assay

- Fixation of living tissue from Ambystoma mexicanum and preparation of tissue sections with paraffin

- Cryosectioning

- siRNA knockdown of large T-Antigen in AC16 cells (first approaches on this cell line)

- Protein expression in COS-7 cells

- Protein expression in $\mathrm{Sf}-9$ and Hi5 cells using Baculo-virus Expression system

Molecular biology

- $\quad P C R, R T P C R, q P C R, P C R$ with UDG-digestion, Primer design

- Agarose Gel-electrophorese; Denaturing gradient gel electrophoresis (DGGE)

- Generation of RNA-Fragments for in situ hybridization, in vitro Transcription of RNA

- Generation of siRNA using Silencer® siRNA Cocktail Kit (RNAse III)

- Mini-, Midi- und Maxi-Prep, Bacmid-Prep

- Restriction digestion, Blunt End reaction, de- und phosphorylation of DNA DNA-Ligation

- Gate-way-cloning

- Gibson-Assembly cloning

- Genotyping of Drosophila melanogaster

- Experience with Micro beads using coupled DNA-Probes; hybridization assay

\section{Microscopy}

- LeicaSP2, Leica SP5, Leica SP5 HMS CARS, Leica GSDIM (Hands-on course)

- Zeiss LSM710

- Zeiss Axiophot Z1

Experience with animal systems:

- Ambystoma mexicanum

- Basic animal keeping, surgical limb removal for regenerative time course studies

- Drosophila melanogaster

- Basic fly husbandry

- Basic genetic combinatorial skills

- Application of Target-system 